\title{
Additive Manufacturing of Bone-forming Composite Implants using Photo-curable Poly(trimethylene carbonate)-based Resins
}

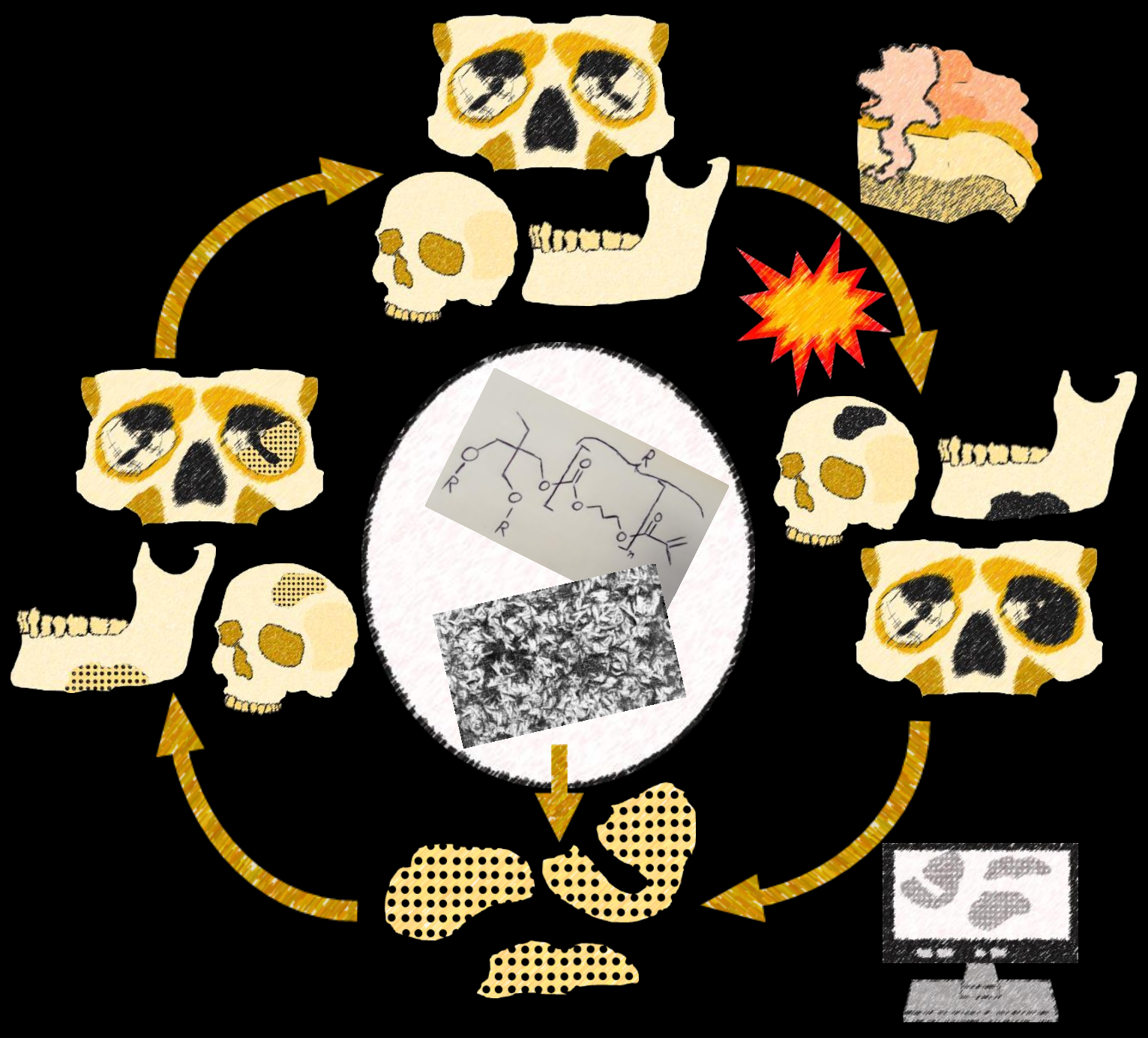

Mike Alexander Geven 


\section{Additive manufacturing of bone- forming composite implants using photo-curable poly(trimethylene carbonate)-based resins}

Mike Alexander Geven 
Additive manufacturing of bone-forming composite implants using photo-curable poly(trimethylene carbonate)-based resins

Mike Alexander Geven

$\mathrm{PhD}$ thesis with references and summaries in English and Dutch University of Twente, Enschede, The Netherlands

February 2018

The research described in this thesis was performed between December 2013 and December 2017 in the research group Biomaterials Science \& Technology of the MIRA institute for Biomedical Technology and Technical Medicine, University of Twente in Enschede, The Netherlands. The research in this thesis was funded by the European Union Seventh Framework program RAPIDOS (NMP-2013-EU-China proposal, project No. 604517).

Printed by Gildeprint Drukkerijen, Enschede, The Netherlands 


\title{
ADDITIVE MANUFACTURING OF BONE- FORMING COMPOSITE IMPLANTS USING PHOTO-CURABLE POLY(TRIMETHYLENE CARBONATE)- BASED RESINS
}

\section{DISSERTATION}

\author{
to obtain \\ the degree of doctor at the University of Twente, \\ on the authority of the rector magnificus, \\ Prof. Dr. T.T.M. Palstra \\ on account of the decision of the graduation committee, \\ to be publicly defended \\ on Wednesday the $9^{\text {th }}$ of May, 2018 at 14:45
}

by

\section{Mike Alexander Geven}

born on the $31^{\text {st }}$ of July, 1988

in Doetinchem, The Netherlands 
This thesis has been approved by the supervisors:

Prof. Dr. D.W. Grijpma

Dr. D. Eglin

(C) 2018 Mike Alexander Geven

ISBN: 978-90-365-4543-3 
Graduation committee:

Chair

Prof. Dr. Ir. J.W.M. Hilgenkamp

Supervisor

Prof. Dr. D.W. Grijpma

Supervisor

Dr. D. Eglin

Members

Prof. Dr. J.D. de Bruijn

University of Twente, The Netherlands

Prof. Dr. Ir. P. Jonkheijm

University of Twente, The Netherlands

Prof. Dr. Ir. T.H. Smit

Academic Medical Center Amsterdam, The Netherlands

Prof. Dr. G. Vozzi

University of Pisa, Italy

Dr. O. Guillaume

AO Foundation, Switzerland 


\section{Publications}

\section{Published}

Chapter 3: Mike A. Geven, Davide Barbieri, Huipin Yuan, Joost D. de Bruijn and Dirk W. Grijpma,

Preparation and mechanical properties of photo-crosslinked poly(trimethylene carbonate) and nano-hydroxyapatite composites. Clinical Hemorheology and Microcirculation, 2015, 60(1), 3-11

Chapter 4: Mike A. Geven, Viktor Varjas, Lukas Kamer, Xinjiang Wang, Jian Peng, David Eglin and Dirk W. Grijpma, Fabrication of patient specific composite orbital floor implants by stereolithography. Polymers for Advanced Technologies, 2015, 26(12), 1433 1438

Chapter 6: Olivier Guillaume, Mike A. Geven, Dirk W. Grijpma, Ting-Ting Tang, Ling Qin, Huipin Yuan, Robert G. Richards and David Eglin, Poly(trimethylene carbonate) and nano-hydroxyapatite porous scaffolds manufactured by stereolithography. Polymers for Advanced Technologies, 2017, 28(10), 1219-1225

Chapter 7: Olivier Guillaume, Mike A. Geven, Christoph M. Sprecher, Vincent A. Stadelmann, Dirk W. Grijpma, Ting-Ting Tang, Ling Qin, Yuxiao Lai, Mauro Alini, Joost D. de Bruijn, Huipin Yuan, Robert G. Richards and David Eglin, Surface-enrichment with hydroxyapatite nanoparticles in stereolithography-fabricated composite polymer scaffolds promotes bone repair. Acta Biomaterialia, 2017, 54, 386-398

Chapter 8: Mike A. Geven, Christoph M. Sprecher, Olivier Guillaume, David Eglin and Dirk W. Grijpma, Micro-porous composite scaffolds of photo-crosslinked poly(trimethylene carbonate) and nano-hydroxyapatite prepared by low temperature extrusion-based additive manufacturing. Polymers for Advanced Technologies, 2017, 28(10), 1226-1232

\section{Submitted for publication}

Chapter 9: Mike A. Geven, Anna Lapomarda, Olivier Guillaume, Christoph M. Sprecher, David Eglin, Giovanni Vozzi and Dirk W. Grijpma, Osteogenic differentiation of hBMSCs on porous photo-crosslinked poly(trimethylene carbonate) and nano-hydroxyapatite composites. 


\section{Contents}

Chapter 1 General introduction 1

Chapter 2 Additive manufacturing of composite structures for the restoration of bone tissue

Chapter 3 Preparation and mechanical properties of photo-crosslinked poly(trimethylene carbonate) and nano-hydroxyapatite composites

Chapter 4 Fabrication of patient specific composite orbital floor implants by stereolithography

Chapter 5 The in vitro degradation of photo-crosslinked poly(trimethylene carbonate) and nano-hydroxyapatite composites

Chapter 6 Poly(trimethylene carbonate) and nano-hydroxyapatite porous scaffolds manufactured by stereolithography

Chapter 7 Surface-enrichment with hydroxyapatite nanoparticles in stereolithography-fabricated composite polymer scaffolds promotes bone repair

Chapter 8 Micro-porous composite scaffolds of photo-crosslinked poly(trimethylene carbonate) and nano-hydroxyapatite prepared by low temperature extrusion-based additive manufacturing

Chapter 9 Osteogenic differentiation of hBMSCs on porous photo-crosslinked poly(trimethylene carbonate) and nano-hydroxyapatite composites

Appendix A Tensile properties of photo-crosslinked poly(trimethylene carbonateco- $\varepsilon$-caprolactone) and nano-hydroxyapatite composites

Appendix B Icariin-releasing formulations for applications in bone restoration

Appendix C Composites based on photo-crosslinked poly(trimethylene carbonate) and microspheres

Outlook

Summary

Samenvatting 


\section{Chapter 1 - General introduction}

Bone is a natural composite material consisting of collagen type-I fibrils and carbonated nano-hydroxyapatite particles, which are bundled into composite fibers. Two types of bone can be discerned, cortical (dense) and cancellous. Cortical bone is comprised of osteons consisting of a central Haversian canal containing blood vessels and nerves which are surrounded by lamellae of bone[1-4]. Cancellous bone consists of trabeculae surrounded by bone marrow, and is therefore less dense. In both cortical as well as cancellous bone, canaliculi are connecting bone cells within the osseous matrix. This allows for vascularization and for cell migration within the bone matrix[1]. Bone is continuously remodeled through the action of osteoclasts which resorb bone in an acid microenvironment and by osteoblasts which lay down de novo bone matrix.

Traumatic injury, tumor removal or pathological diseases can result in bone fractures or bone defects. Generally, bone is capable of perfectly restoring itself subsequently. Blood and nutrient supply is of utmost importance for this natural healing of bone defects and it is therefore necessary that local vascular systems are preserved close to bone defects[1, 5]. After formation of a hematoma from blood, platelets express growth factors and initiate a healing cascade consisting of inflammation, cytokine signaling and growth factor expression, allowing for recruitment of osteoprogenitor cells and for blood vessel formation[1]. After hematoma formation, a soft callus is formed. This consists of cartilaginous tissue connecting the bony fragments and the bony edges of a defect. The soft callus is mineralized over time through the action of osteoblasts[6]. The hard callus that is formed through this process subsequently maturates into bone tissue through osteoblast and osteoclast activity. Although this elegant process allows for restoration of most bone fractures and defects, 5 to $10 \%$ of patients experience complications due to impaired fracture healing[5]. In some of these cases the size of a fracture or defect can be such that the natural healing capacity of bone is not sufficient to restore it. Such defects are of 'critical size'.

Currently, such critical size defects are restored by bone grafting procedures in which the defect is filled by autogenic, allogenic or xenogeneic bone. Bone is the second most transplanted tissue following blood in the first place, and it is estimated that worldwide 2.2 million bone grafting procedures are performed annually[4, 5]. Autogenous corticocancellous bone grafts taken from the iliac crest, fibula, scapula or radius are mainly used[7]. Grafting procedures are not ideal however. Additional surgical procedures on the patient are required for autogenic transplantations, there is a risk of donor site morbidity, a limited availability of autografts and a prolonged hospitalization is required[3, 8]. For allografts and xenografts there is an additional risk of disease transmittance, graft rejection and sub-optimal integration with surrounding tissue[1, 3-5]. Furthermore, the shape of any type of graft may not fit specific defects and shaping options are suboptimal. 
Aside from these drawbacks, natural bone grafts are still most effective in the restoration of large bone defects. Due to the combination of osteoconduction, osteoinduction and osteogenicity of natural graft materials, no synthetic material (e.g. alloys, ceramics, polymers and composites thereof) has surpassed its effectiveness to date. Osteoconductivity promotes cell attachment, migration and proliferation and therefore allows for bone ingrowth. Osteoinduction promotes stem cells or osteoprogenitor cells towards the osteoblast lineage, and accordingly stimulates the formation of bone. Osteogenicity refers to the presence and delivery of osteogenic stem cells or progenitor cells from within the implant[3, 5, 9, 10].

Ideally, synthetic implants for the restoration of critical size bone defects possess all advantages of natural bone grafts without their disadvantages. They should therefore be biomimetic and bioresponsive and thus provide the osteoconductive, osteoinductive and osteogenic environment which is lacking in critical size defects[1]. In addition, the ideal synthetic bone graft material is readily shapeable in order to fit any defect (patientspecificity) and possesses an internal architecture suitable for bone ingrowth and vascularization. This requires for the presence of large pores with a diameter of at least 100 $\mu \mathrm{m}$ and preferably over $300 \mu \mathrm{m}$. Smaller pores can furthermore enhance bone ingrowth by providing additional surface roughness, enlarging the concentration of proteins locally and by improving the nutrient supply and waste removal throughout the implant[10-12]. Roughness features in the nanometer range have shown to be effective in promoting cell attachment and differentiation[3]. The degradability of implant structures is another important aspect as permanent implants result in stress-shielding for de novo bone and a lasting foreign body response[1,8]. The degradation of an implant should be controlled so that it provides a temporary support structure without premature mechanical failure[1].

\section{Aims \& Outline of this thesis}

In this work we describe the development of a novel composite implant material based on photo-crosslinked methacrylate end-group functionalized poly(trimethylene carbonate) and nano-hydroxyapatite (nHA). Poly(trimethylene carbonate) (PTMC) is an amorphous and flexible polymer that degrades through enzymatic and oxidative pathways. Since no acidic degradation products are formed during the degradation of PTMC, we hypothesize that it is a valuable candidate polymer for use in bone tissue engineering[13-15]. The nHA is a calcium phosphate that is similar to the mineral component in bone. It was shown to imbue osteoinductive properties to composites with poly(D,L-lactide)[16].

The aim of this work was to not only develop the photo-crosslinked composites of PTMC and nHA, but to also develop methods to prepare implantable composite structures from these materials by means of additive manufacturing techniques. This would allow for preparation of patient-specific implants with an internal architecture suitable for bone restoration. Additionally, the composite properties (mechanical properties, hydrophilicity, surface morphology, degradation characteristics i.a.) and their performance in vitro in cell culture experiments and in vivo in animal models was evaluated. 
In Chapter 2 several additive manufacturing techniques suitable for composite preparation are discussed. The focus is on the implications of processing a composite feedstock by these techniques on the processing conditions needed. Additionally, an overview is given on previous work that has been performed on the additive manufacturing of composites for bone tissue restoration.

In Chapter 3 composites of PTMC and nHA photo-crosslinked in bulk are introduced. The incorporation of nHA in a photo-crosslinked PTMC matrix and the effect of the incorporation on the photo-crosslinking and on the mechanical properties of the composites are explored in this chapter.

Chapter 4 shows that photo-crosslinked composites of PTMC and nHA can be produced by stereolithography. Using this additive manufacturing technique, patient-specific implants can be fabricated. In this chapter the preparation of implants for orbital floor reconstruction is described, showing that composites with a complex and patient-specific shape can be prepared. The mechanical properties and hydrophilicity of these composites are furthermore characterized.

In Chapter 5 the in vitro degradation of photo-crosslinked PTMC and nHA composite films manufactured by stereolithography is investigated. The effect of nHA content and different degradation media is shown. Surface morphological changes, mass loss and compositional changes are observed to elucidate the degradation characteristics of the composites. It is shown that the degradation in enzymatic and acidic medium strongly depends on the nHA content of the composites.

The properties and cytocompatibility of composite structures with a porous internal architecture and varying nHA content prepared by stereolithography is investigated in Chapter 6. Human bone marrow mesenchymal stem cells (hBMSCs) are seeded on the porous structures and their adherence and activity is investigated

Porous composite structures are further characterized in Chapter 7. The surface structure and nHA distribution in the structures is investigated along with the protein adhesion, calcium release and osteogenic differentiation of hBMSCs. Ultimately, the implantation of porous composite structures in rabbit calvarial defects in vivo is described.

Chapter $\mathbf{8}$ describes the fabrication of porous composites with a multiscale porosity by use of low temperature extrusion-based additive manufacturing and temperature induced phase separation. It is shown that drugs may be incorporated in composites prepared by this method. The mechanical properties and drug release are characterized and it is shown that defined three dimensional structures can be prepared.

In Chapter 9 porous composites of photo-crosslinked PTMC and nHA are prepared by a temperature induced phase separation. The porosity of these composites is varied and the effect of the porosity on surface morphology, mechanical properties, protein adhesion, calcium release and the osteogenic differentiation of hBMSCs is investigated. 


\section{References}

1. Fernandez-Yague, M.A., S.A. Abbah, L. McNamara, D.I. Zeugolis, A. Pandit, and M.J. Biggs, Biomimetic approaches in bone tissue engineering: Integrating biological and physicomechanical strategies. Advanced Drug Delivery Reviews, 2015. 84: p. 1-29.

2. Hutmacher, D.W., J.T. Schantz, C.X. Lam, K.C. Tan, and T.C. Lim, State of the art and future directions of scaffold-based bone engineering from a biomaterials perspective. Journal of Tissue Engineering \& Regenerative Medicine, 2007. 1(4): p. 245-260.

3. McMahon, R.E., L. Wang, R. Skoracki, and A.B. Mathur, Development of nanomaterials for bone repair and regeneration. Journal of Biomedical Materials Research Part B Applied Biomaterials, 2013. 101(2): p. 387-397.

4. Fu, Q., E. Saiz, M.N. Rahaman, and A.P. Tomsia, Toward Strong and Tough Glass and Ceramic Scaffolds for Bone Repair. Advanced Functional Materials, 2013. 23(44): p. 5461-5476.

5. Van der Stok, J., E.M. Van Lieshout, Y. El-Massoudi, G.H. Van Kralingen, and P. Patka, Bone substitutes in the Netherlands - a systematic literature review. Acta Biomaterialia, 2011. 7(2): p. 739-750.

6. Ghiasi, M.S., J. Chen, A. Vaziri, E.K. Rodriguez, and A. Nazarian, Bone fracture healing in mechanobiological modeling: A review of principles and methods. Bone Repair, 2017. 6: p. 87-100.

7. Schieker, M., H. Seitz, I. Drosse, S. Seitz, and W. Mutschler, Biomaterials as Scaffold for Bone Tissue Engineering. European Journal of Trauma, 2006. 32(2): p. 114-124.

8. Kinoshita, Y. and H. Maeda, Recent developments of functional scaffolds for craniomaxillofacial bone tissue engineering applications. ScientificWorldJournal, 2013. 2013: p. 863157.

9. Kolk, A., J. Handschel, W. Drescher, D. Rothamel, F. Kloss, M. Blessmann, M. Heiland, K.D. Wolff, and R. Smeets, Current trends and future perspectives of bone substitute materials - from space holders to innovative biomaterials. Journal of Craniomaxillofacial Surgery, 2012. 40(8): p. 706-718.

10. Habibovic, P. and K. de Groot, Osteoinductive biomaterials--properties and relevance in bone repair. Journal of Tissue Engineering \& Regenerative Medicine, 2007. 1(1): p. 25-32.

11. Bose, S., M. Roy, and A. Bandyopadhyay, Recent advances in bone tissue engineering scaffolds. Trends in Biotechnology, 2012. 30(10): p. 546-554.

12. Tarafder, S., V.K. Balla, N.M. Davies, A. Bandyopadhyay, and S. Bose, Microwave-sintered $3 D$ printed tricalcium phosphate scaffolds for bone tissue engineering. Journal Tissue Engineering \& Regenerative Medicine, 2013. 7(8): p. 631-641. 
13. Zhang, Z., R. Kuijer, S.K. Bulstra, D.W. Grijpma, and J. Feijen, The in vivo and in vitro degradation behavior of poly(trimethylene carbonate). Biomaterials, 2006. 27(9): p. 1741-1748.

14. Chapanian, R., M.Y. Tse, S.C. Pang, and B.G. Amsden, The role of oxidation and enzymatic hydrolysis on the in vivo degradation of trimethylene carbonate based photocrosslinkable elastomers. Biomaterials, 2009. 30(3): p. 295-306.

15. Yang, L.-Q., J. Li, W. Zhang, Y. Jin, J. Zhang, Y. Liu, D. Yi, M. Li, J. Guo, and $\mathrm{Z}$. $\mathrm{Gu}$, The degradation of poly(trimethylene carbonate) implants: The role of molecular weight and enzymes. Polymer Degradation \& Stability, 2015. 122: p. 77-87.

16. Barbieri, D., A.J. Renard, J.D. de Bruijn, and H. Yuan, Heterotopic bone formation by nano-apatite containing poly(D,L-lactide) composites. European Cells \& Materials, 2010. 19: p. 252-261. 


\title{
Chapter 2 - Additive manufacturing of composite structures for the restoration of bone tissue
}

Mike A. Geven and Dirk W. Grijpma

Department of Biomaterials Science and Technology, University of Twente, Enschede, The Netherlands

\begin{abstract}
Large bone defects are challenging to reconstruct and novel biologically active materials are being developed for this purpose. Composites of polymers or polymer networks with inorganic particles have been applied in the past with success. The efficacy of composites in bone regeneration may be further improved by the use of additive manufacturing techniques for the fabrication of implants. These techniques allow for highly porous structures with an interconnected porosity to be fabricated. Several additive manufacturing techniques are suitable for composite processing, and a multitude of composite materials has been developed. In this review we discuss additive manufacturing techniques that have been applied for the fabrication of composites implant structures for bone tissue regeneration. We furthermore discuss the implications of processing composites, methods to design composite structures for additive manufacturing and provide a literature overview of results that have been achieved with additively manufactured composites for bone tissue restoration.
\end{abstract}




\section{Introduction}

Additive manufacturing (AM) has attracted increasing attention for the preparation of bioactive implantable devices. For tissue regeneration applications, well-designed structures with high porosity, high interconnectivity and a defined pore size and pore geometry are required for effective cell migration, nutrient supply and extracellular matrix (ECM) production within the structures[1-4]. Use of conventional techniques such as porogen leaching, gas foaming or phase separation methods, results in a broad pore size distribution, varying pore geometry and generally low interconnectivity[1, 2]. Especially for bone defects, the importance of structures with high porosity and interconnected pores has been demonstrated frequently in the past years[5-8]. This makes AM especially attractive for the fabrication of structures for bone tissue restoration.

In AM, scaffold building material or energy is applied at pre-defined locations in a layerby-layer manner to form a three dimensional structure. This temporo-spatial control over material deposition or immobilization makes AM ideal for the fabrication of well-defined porous constructs. Furthermore, when combined with three dimensional medical imaging such as computed tomography (CT) it allows for the fabrication of patient specific implant structures as illustrated in figure 1 for a bone defect. The data from CT scans can readily be converted into a computer aided design (CAD) model in standard tessellation language (STL). Such an STL file may then be adjusted by modelling software to apply the desired porosity or supporting structures if needed. The resulting STL file is virtually sliced into 2D layers and these serve as a template for the layer-by-layer fabrication of the desired 3D construct as is illustrated in figure $1[9,10]$.
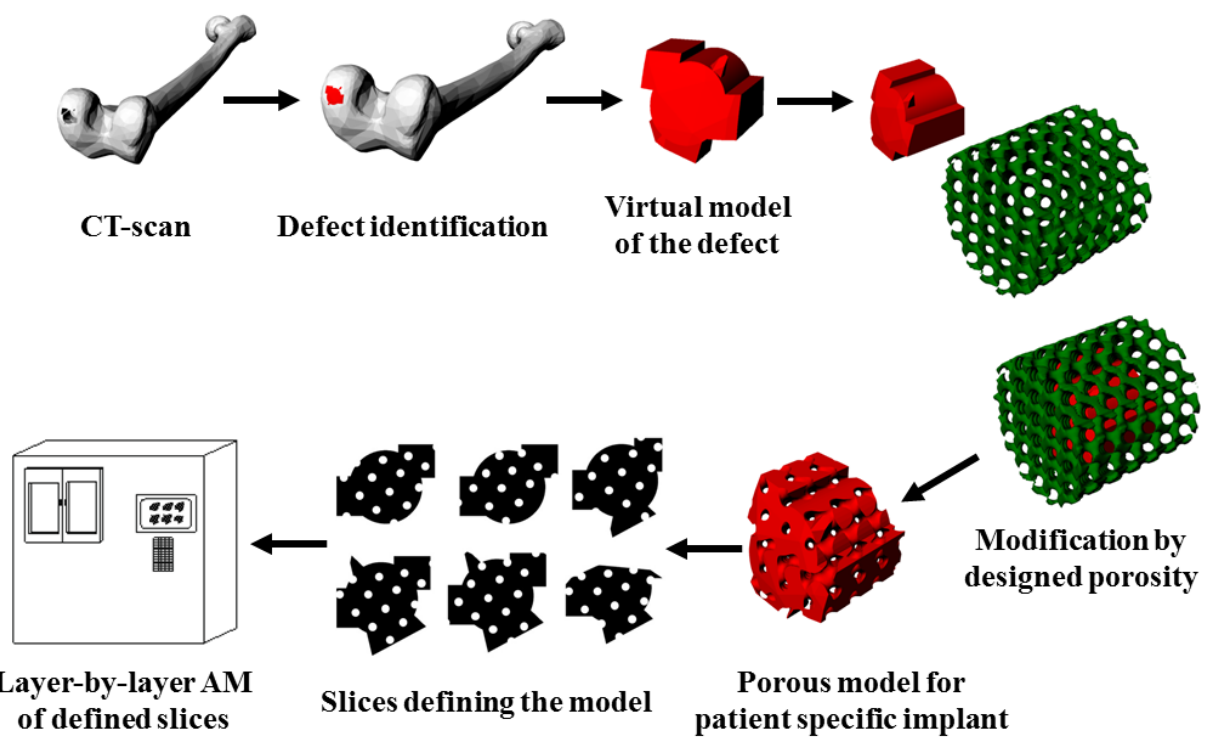

Figure 1. Steps involved in additive manufacturing of patient specific implant structures. 
Due to the attractiveness of AM for the preparation of tissue engineering structures, a wide array of publications and reviews on AM of polymer structures is available[1-4, 11-17]. Although implant structures prepared only from polymers have also been applied successfully for bone tissue engineering purposes, they do not provide direct biological cues for bone regeneration. Research has therefore focused on methods to improve the efficacy of three dimensional structures for bone tissue restoration. Such methods include the release of growth factors or promotive medicines, surface modification, the inclusion of cells or the addition of an inorganic phase to the polymer to form a composite[18-21]. The latter is described in different reviews on the fabrication and biomedical application of tissue engineering scaffolds prepared by AM. An overview of literature specifically dealing with bioactive composites prepared by AM for bone tissue engineering is still lacking however. Here we provide such an overview, along with a description of AM techniques that have been applied for composite structure fabrication, implications of processing composite materials with these techniques and methods to design porous structures.

\section{Additive manufacturing techniques for composite fabrication}

A variety of AM techniques have been applied for the fabrication of composite scaffolds for bone tissue regeneration. An overview of these techniques is presented in table 1 . In the following paragraphs we will discuss the working principle of these techniques as well as the consequences of processing composite materials.

Table 1. An overview of AM techniques applied for preparation of composite structures.

\begin{tabular}{lcc}
\hline AM technique & $\begin{array}{c}\text { Typical } \\
\text { resolution }\end{array}$ & Materials \\
\hline 3D printing & $100 \mu \mathrm{m}$ & Polymers, inorganics \\
Stereolithography & $15-300 \mu \mathrm{m}$ & Photo-polymerizable polymers \\
Fused deposition modelling & $250-500 \mu \mathrm{m}$ & Polymers, waxes \\
Precision extrusion deposition & $100-250 \mu \mathrm{m}$ & Polymers, slurries \\
Low temperature deposition & $250-500 \mu \mathrm{m}$ & Polymers, biological material, \\
modelling & & slurries \\
3D Bioplotting & $250-400 \mu \mathrm{m}$ & Polymers, biological material \\
Selective Laser sintering & $50-100 \mu \mathrm{m}$ & Polymers, inorganics \\
\hline
\end{tabular}

\subsection{D printing}

The 3D printing technique is an additive manufacturing method that fabricates structures by selective adhering of polymeric- or inorganic powder into 3D structures in a layer-by-layer fashion. This is illustrated in figure 2. During fabrication, the powder is supplied and spread in a thin layer over a build platform using a powder supply and roller. It is subsequently fused in 2D patterns by dispensing of a binder on top of the powder layer. The build platform is thereafter lowered and fresh powder is spread on top of the fused pattern. Subsequent binding of fresh powder results in generation of the next layer of the 
construct. This process is similar to that of selective laser sintering (figure 7) which we will discuss in more detail in a following paragraph. The binder in 3D printing may consist of organic solvents, crosslinking agents, polymer solution or colloidal particles[1, 22]. Generally, fabricated structures are porous and may need reinforcement by impregnation of binder after removal of excess powder to improve their mechanical stability[10].

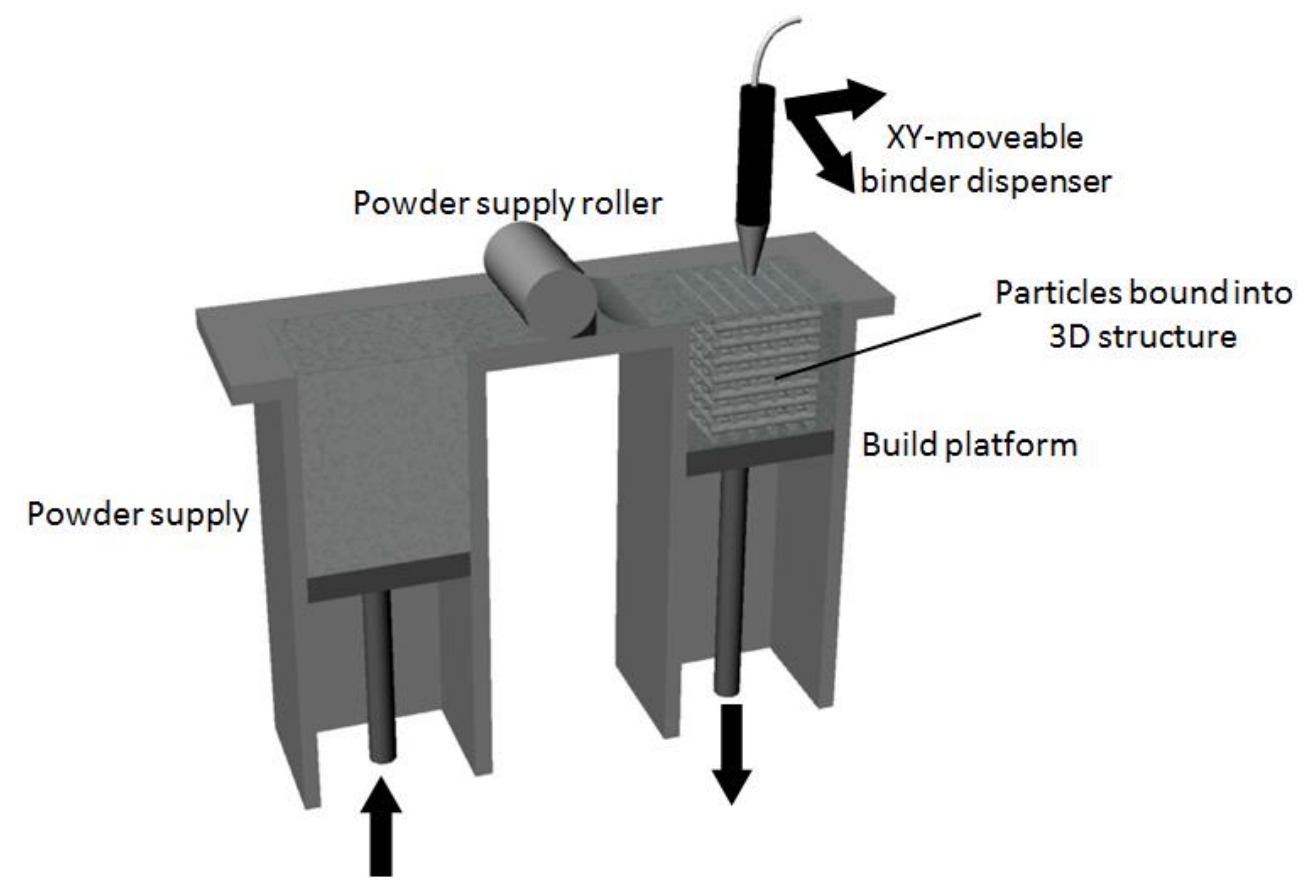

Figure 2. Schematic of the $3 D$ printing process

\subsection{Stereolithography}

Stereolithography was the first commercially available AM technique, developed by 3D Systems in 1986[9]. The technique is based on a free radical photo-polymerization, initiated by a photo-sensitive initiator molecule. Structures are prepared using a photocurable resin, which is a liquid that solidifies following irradiation with a light source upon photo-polymerization. It generally consists of the initiator, a species that can be polymerized by free radical polymerization and, if needed, a diluent. Figure 3 shows schematic representations of two different stereolithography apparatuses. In a bottom-up apparatus, the base of a structure is formed by polymerization on the top side of a moveable platform, just below the resin surface. A thin layer is polymerized in a $2 \mathrm{D}$ pattern drawn by a guided laser light beam. The fabrication platform thereafter descends and subsequent polymerization of patterns on top of previous layers forms the desired construct $[9,13]$. In the top-down approach, the resin-containing basin has a transparent non-sticking bottom. The topmost part of the $3 \mathrm{D}$ construct is initially formed by illumination through the 
transparent bottom. In this case, the fabrication platform is positioned just above the transparent bottom and rises in steps after formation of subsequent $2 \mathrm{D}$ patterns. In this approach additional forces are placed on the as-fabricated structure, as it needs to be separated from the bottom. The structures therefore need sufficient mechanical resilience to withstand these forces. Advantages of the top-down approach over the bottom-up approach include the lower amount of resin required, the absence of oxygen inhibition due to polymerization strictly below the resin surface and the smoother surfaces of the constructs that are obtained[9]. Since the 1990's several improvements have been realized to improve the resolution of the stereolithography technique[23-25]. In a recent report of Tumbleston et al. a resolution of $1 \mu \mathrm{m}$ was readily achieved[26].
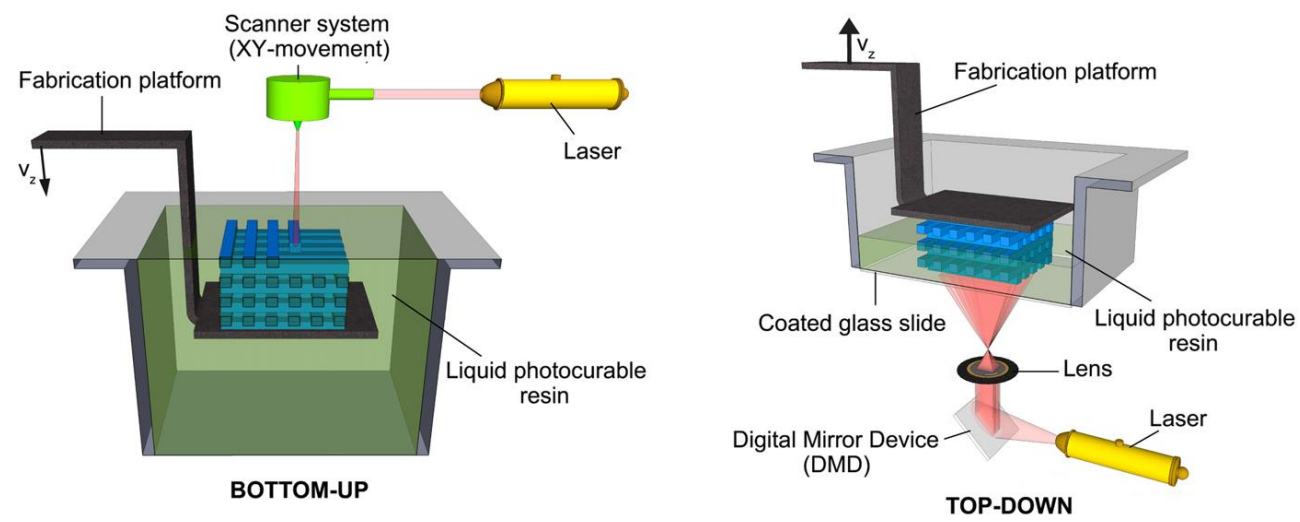

Figure 3. Schematic representation of stereolithographic working principles. Adapted from [13].

For the fabrication of composite structures by stereolithography, an inorganic phase can be dispersed into the photo-curable resin. The effect of the inorganic particles on the light absorption and scattering in the resin needs to be considered in that case. Any absorption and scattering by the particles at the wavelength used to initiate the photo-polymerization process will affect the curing depth and width[27]. It therefore influences the adhesion of newly polymerized patterns onto the previous layers and the XY-resolution. In order to guarantee the integrity of structures, the light intensity and irradiation times employed in the stereolithography process need to be adjusted for specific particles and the particle content. The particle size should furthermore be smaller than the pattern thickness so that the pattern is not disrupted[9, 27].

Furthermore, the viscosity of resins may not be excessively high since the resin needs to flow over or underneath the build platform. The viscosity must be high enough to prevent significant sedimentation of particles within fabrication times, however. It has been previously reported that resin viscosity should not exceed $5 \mathrm{~Pa} \cdot \mathrm{s}$ [27]. However, this strongly depends on the setup and machine settings that are applied. It has been reported that resins based on suspensions of nano-hydroxyapatite in photo-polymer solutions with a viscosity of up to $72 \mathrm{~Pa} \cdot \mathrm{s}$ yielded satisfactory fabrication results[28]. Reactive and non- 
reactive diluents can be applied to control the viscosity of resins. When adding diluents, shrinkage of fabricated structures and their effect on the mechanical properties of asfabricated structures need to be considered.

\subsection{Fused deposition modelling}

Fused deposition modelling (FDM) has been available commercially since 1991, when it was introduced on the market by Stratasys[10]. In FDM, thermoplastic materials are meltextruded as fibers to form a 2D pattern on a fabrication platform. Generally, 2 materials are utilized during the build process, one functioning as a support material whereas the other composes the actual construct material. A schematic representation of an FDM system is depicted in figure 4. Fibers of thermoplastic are fed into heated tips and molten polymer is deposited in a pattern through XY-movement of the tip. Movement of the build platform in the Z-direction allows for subsequent deposition and fusing of subsequent 2D layers. The eventual characteristics of the construct, such as the resolution of the built layers, porosity, mechanical properties and anisotropy, are determined by the processing parameters. Tip temperature, tip diameter, movement speed of the tip, extrusion rate and build direction can be altered to affect these characteristics[29, 30].

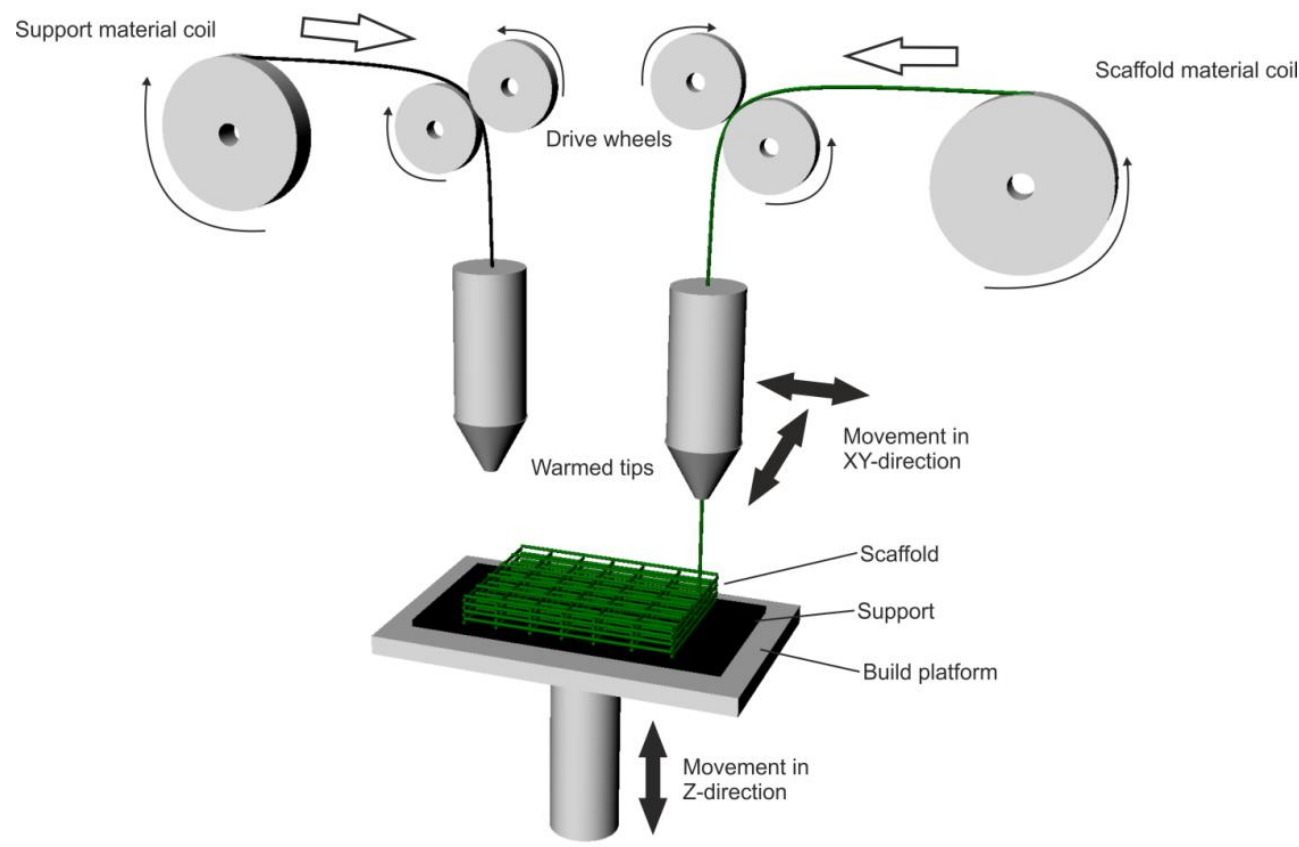

Figure 4. Schematic of the FDM principle.

In order to process composite materials by FDM, composite filaments need to be produced to be fed into the filament extruder. Homogeneity of these filaments is of importance in order to fabricate homogeneous structures. Inhomogeneity in the form of particle 
aggregates can cause clogging of the tips and therefore needs to be avoided. It is also important to consider the increase of the viscosity of the polymer melt upon particle addition. Increasing particle contents will elevate the viscosity and encumber the fiber extrusion. Therefore the amount of particles that may be added is limited. It has been shown that addition of a surfactant during the pellet or filament production for FDM can overcome these issues by increasing the interfacial bonding between the polymer and inorganic phase[31, 32].

\subsection{Extrusion-based 3D printing}

The term 'extrusion-based 3D printing' is currently used to describe a set of techniques in which material (polymer melt, polymer solution or slurries) are extruded from a cartridge. The technique to extrude these materials may vary and can be driven pneumatically, by a piston or by a screw as illustrated in figure 5. In this paragraph, three extrusion-based 3D printing techniques are discussed.
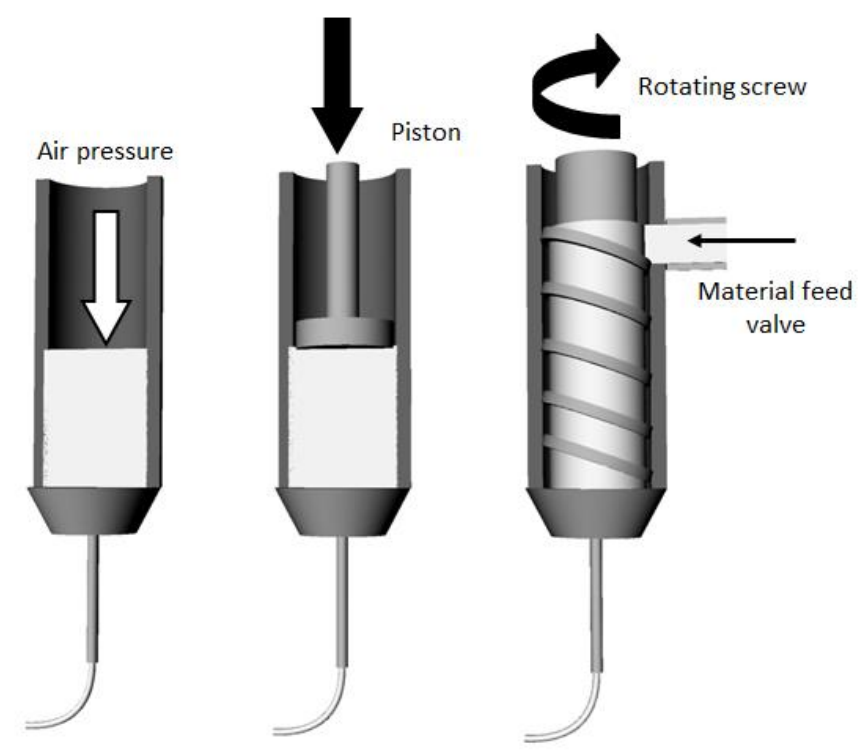

Figure 5. Illustration of different extruding cartridges that may be used for 'extrusion-based 3D printing'.

\section{Precision extrusion deposition}

Since 2002 a modification of the FDM process was introduced which enhanced the accuracy of the process. These enhancements culminated in a process referred to as precision extrusion deposition (PED). The improvements are realized by the use of pellet screw extruders which allow for meticulous control over the amount of material that is extruded. Furthermore, the extruder is synchronized with a precision system controlling XY-movement of the build platform. This allows for an exact control over the fiber diameter and positioning[33]. As with FDM, the homogeneity of the feedstock for PED is 
of importance for the fabrication of homogeneous structures. Composite pellets for the screw extruder in PED must therefore be prepared with minimal amount of particle aggregates. The viscosity increase of the polymer melt is another consideration, although screw extruders may be adjusted to accommodate a broad range of viscosities.

\section{Low temperature deposition modelling}

Low temperature deposition modelling (LDM, also named low temperature extrusion-based additive manufacturing) is a rather novel technique first reported by Xiong et al. In 2002[34]. This technique operates on a similar principle as PED. In LDM materials are deposited from XY-movable tips onto a platform that moves in the Z-direction. Generally cartridges are used from which material expulsion is controlled by air pressure or a piston, although screw extruders may also be applied.

In LDM the deposited materials generally consist of viscous polymers, polymer solutions or slurries that can be extruded at room temperature or at slightly elevated temperatures[3436]. This differentiates the technique from FDM and PED, where relatively high temperatures are required for the melt-processing of polymers. The discharged materials in LDM experience a phase change or phase separation directly after deposition to immobilize them. This is generally caused by a temperature change by cooling the build platform or reducing the environmental temperature.

For the fabrication of composite scaffolds by LDM, similar considerations need to be taken into account as with FDM or PED. In LDM the viscosity increase of feedstock with increasing particle contents can be counteracted by the use of diluents however. Although the diluent content cannot be increased limitlessly, as the stability of printed structures can be affected by it, it does offer increased flexibility in the processing of materials. The temperature of the tip can furthermore be used to control the viscosity of the printing material to some extent.

\section{D-Bioplotting}

The $3 \mathrm{D}-\mathrm{BioPlotter}{ }^{\mathrm{TM}}$ is a $3 \mathrm{D}$ dispensing system in which viscous materials such as polymer solutions, slurries or dispersions of biological material are deposited on the surface of a platform that is submerged in a non-solvent $[13,22]$. A schematic of this AM technique is given in figure 6. The 3D-bioplotting technique was developed by Landers et al. using a modified computer numerical control (CNC) milling machine[37]. Building of scaffolds by this technique proceeds via a pressurized nozzle that can move in three dimensions while the build surface is stationary. Although this is similar to PED and LDM, material in the 3D-BioPlotter ${ }^{\mathrm{TM}}$ is generally only deposited by pneumatic systems and the movement of the nozzle in three dimensions is characteristic. The deposition of material on a build platform in a non-solvent is another key distinctive feature of this process. The non-solvent causes solidification of deposited material and can be chosen to be of similar density as the plotted material, yielding buoyancy compensation. In the latter case no support materials are required and it is especially useful for low viscosity materials to prevent flowing after 
deposition. Furthermore, depending on the scaffold material utilized, aqueous media may be used during fabrication which is beneficial for the construction of structures with biological material.

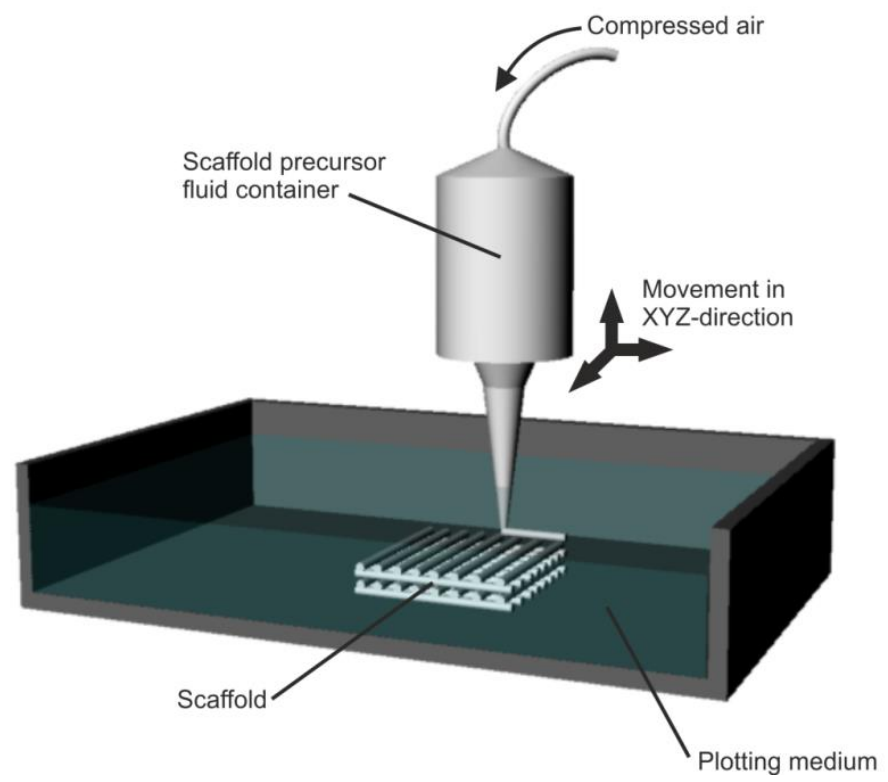

Figure 6. Schematic of a 3D-Bioplotting machine.

For the processing of composites, mainly the viscosity increase caused by the particles needs to be considered. Since compressed air is utilized to drive material deposition, an increase of the viscosity will affect the required air pressure. Introducing particles into the viscous material that is to be deposited can sharply increase viscosity[38]. The required pressure scales linearly with the viscosity increase[39]. In some cases it may exceed what is practically achievable[40]. As with LDM, use of additional solvent can counteract viscosity increase due to particle addition.

\subsection{Selective laser sintering}

In selective laser sintering (SLS), scaffolds are generally produced from polymer or inorganic powders as depicted in figure 7. As with 3D printing, powder is supplied and spread in a thin layer over a build platform using a powder supply and roller. In SLS it is subsequently irradiated by a laser beam. The beam fuses the powder in a defined pattern. The build platform is thereafter lowered and fresh powder is deposited on top of the fused pattern. Subsequent irradiation of the fresh powder results in generation of the next layer of the construct. In the case of polymer powders, fusion occurs by raising the temperature of the polymer above the melting temperature (semi-crystalline polymers) or just above the glass transition temperature (amorphous polymers) and subsequent cooling. Suitable 
polymers should be readily processed into a powder and should possess a high melting enthalpy and low thermal conduction. Only when the latter two requirements are met, welldefined structures can be built[10].

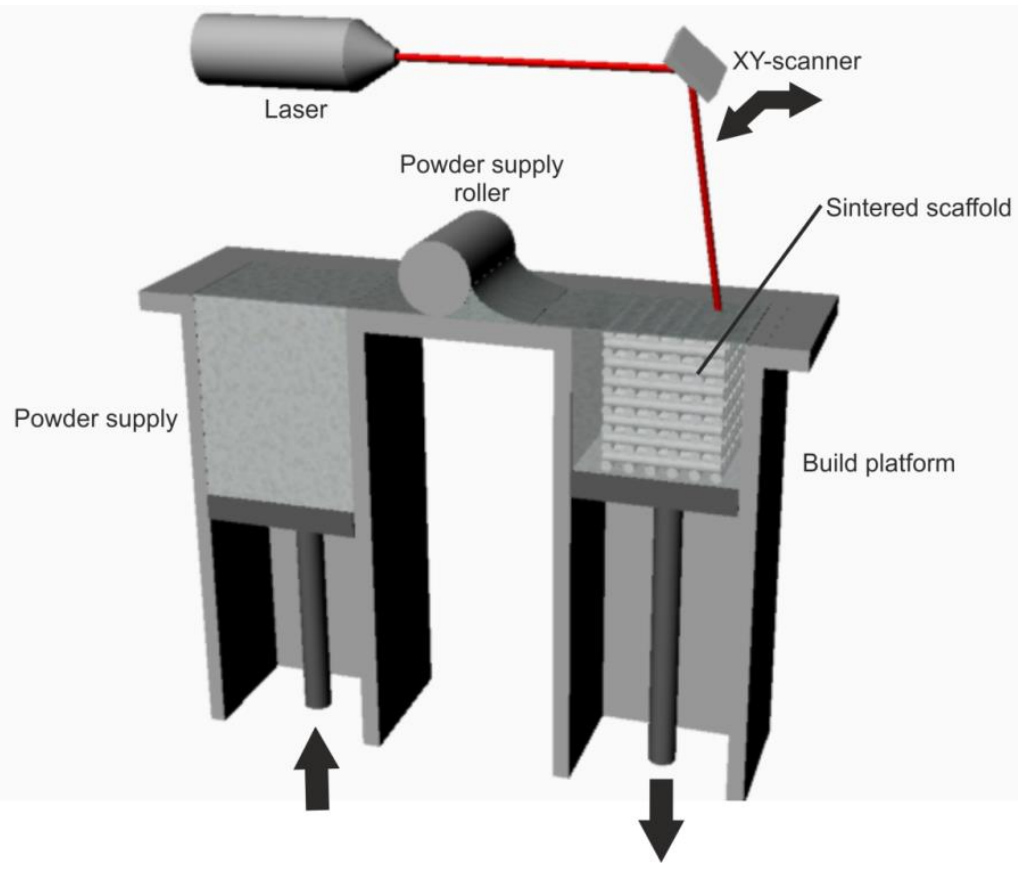

Figure 7. Process schematic of SLS.

Fabrication of composite scaffolds is readily performed by SLS. Polymeric- and inorganic powders with similar particle size in the range of $10-100 \mu \mathrm{m}$ can be mixed and fed into the powder supply for sintering. Generally the laser energy required for sintering a mixed powder will be higher than for the pure polymer powder, due to energy absorption by the inorganic phase and a lower content of the polymer binding the inorganic particles [41-43]. The distance between the lines traced by the path of the laser and the scanning rate can be reduced while the power output of the laser can be increased when inorganic particles are introduced to the feed powder. This increases the energy density that is supplied to the powder to allow for a sufficiently thick layer $(50-100 \mu \mathrm{m})$ of powder to fuse [41, 42].

\section{Design of porous structures for AM}

Structures for the restoration of bone defects require a porosity that allows for bone ingrowth as well as neovascularization[6]. Structures with interconnected pores of a diameter in excess of $300 \mu \mathrm{m}$ are generally regarded as optimal[6]. In order to prepare materials with a well-defined and interconnected internal structure, it is necessary to produce high resolution virtual designs. To prepare such designs manually is a painstaking 
task which is time consuming and requires an excessive amount of computer memory[4446]. Due to the increasing attention towards the use of AM for fabrication of bioactive structures, effort has been put into the efficient design of well-defined porous structures and in reducing the amount of data that needs to be processed. In this paragraph we shall introduce a selection of efficient design approaches. For an extensive review on the design of porous structures for tissue regeneration the reader is referred to an extensive paper by Gianitelli et al.[47].

Several approaches uncouple the internal design of porous structures from the external features of the structure. For example, Starly et al. introduced design approaches in which an outer shape and internal structure were initially defined after which a matching procedure directly generates contour slices based on both structures [44]. This concept is illustrated in figure 8 . The generated contour slices can be directly reproduced in a layerby-layer fashion by AM. Additionally, Starly et al. introduced a design approach based on Lindenmayer systems[45]. In this approach, the internal structure of scaffolds is designed by recursive, space-filling curves. Starly et al. show that this allows for an efficient design and that poly( $\varepsilon$-caprolactone) (PCL) structures can be fabricated using FDM and these designs.

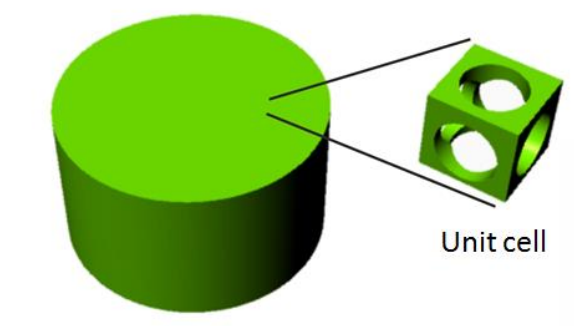

Outer shape of the structure

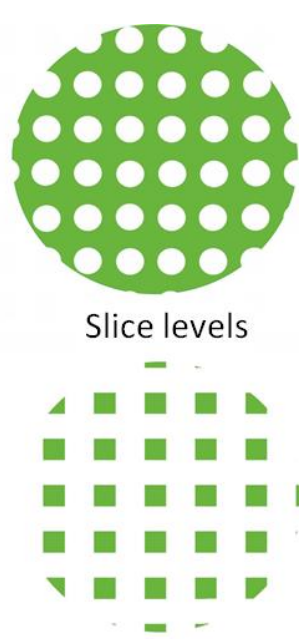

Figure 8. Schematic of the IAD approach for 3D construct modeling. Outer shape of the structure and internal features are separately designed. The outer shape of the structure is filled out with the internal unit cell structure in silico. Intersection points between the outer shape and internal structure and between the internal unit cells are calculated by an iterative process. This allows for direct definition of the slice levels shown on the right (green indicates the material to be fabricated). Repeated layer-by-layer fabrication of the slice levels will yield a construct with a predefined outer shape and an internal porosity defined by the unit cell. Note that the slicing in this example is of a low resolution, defining only the top/bottom of each layer of unit cells (upper slice level depicted) and the mid-section of each layer of unit cells (bottom slice level depicted). Figure adapted from [44].

The use of triply periodic minimal surfaces (TPMS) for the design of scaffolds was introduced in a report of Rajagopalan et al.[46]. The TPMS are periodic in three 
independent directions, extend indefinite, do not intersect themselves and therefore subdivide space into two 'labyrinths'. Examples of such surfaces are depicted in Figure 9. Due to the continuous subdivision of space by TPMS, they can be utilized to define a solid phase and a porous space by filling one of the defined labyrinths. Yoo utilized a method of scaffold preparation based on TPMS without the need for generation of STL files[48, 49]. In this method, TPMS were approximated by nodal equations for the design of the internal architecture of scaffolds. The outer surface of the scaffolds was defined by distance field operations. The scaffold was therefore not defined by a CAD model with detailed internal structure, but solely as the mathematical description of the external and internal structure.

Additively manufactured, three dimensional structures with porosities based on TPMS have been analyzed in detail. For example, the deformation characteristics under compression, of structures with a gyroid-based porosity has been characterized using CT imaging. It was shown that the extent of deformation was affected by the pore size of the structures[50]. Furthermore, the surface curvature in pores of different TPMS structures has been characterized. It was shown that the type of TPMS used to model the porosity directly affects the surface curvature, which may in turn affect the behavior of cells seeded in the porosity of the structures[51]. Using structures with porosity based on gyroid TPMS it was shown that cell distribution inside porous structures can be influenced by porosity and pore size gradients[52]. In addition, by using stereolithography several TPMS-based scaffolds were prepared for use in bone reconstruction, cartilage restoration, annulus fibrosus reconstruction and as biocompatible shape memory implants[9, 53-64].

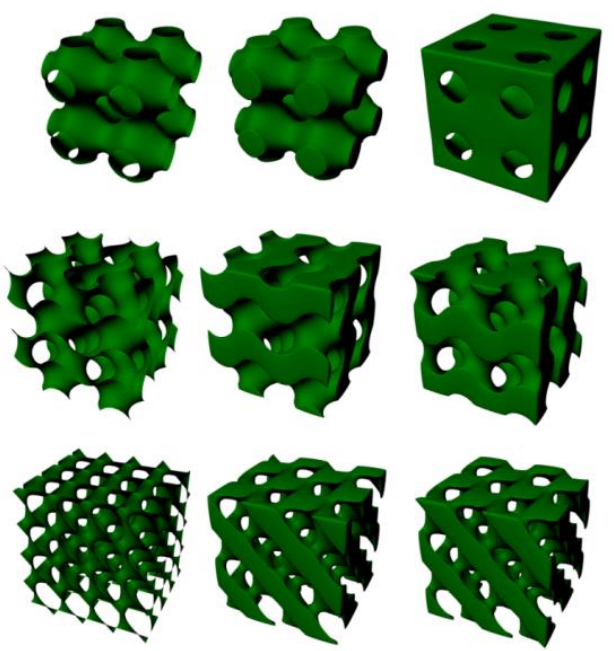

Figure 9. Examples of TPMS and their spacefills. Left column from top to bottom: primitive, gyroid and diamond surface respectively. Middle and right column: spacefills of either one of the spaces defined by the respective TPMS. 


\section{Composites for bone tissue engineering prepared by AM}

The natural composite structure of bone consists of collagen type I fibrils loaded with nanohydroxyapatite[65]. This is an excellent composite material with high stiffness and toughness. It has inspired the scientific community to mimic this environment by preparing synthetic composites. Indeed, such composites have been shown to possess better osteoconductivity than polymer scaffolds coated in calcium phosphates [66, 67]. The release of calcium ions, surface roughness, micro-porosity and the mechanical properties of the composite material imbued by the calcium phosphates play a vital role in its performance in osteogenic differentiation and bone regeneration in 2D and 3D[68-74]. Here we will discuss several research topics that have been investigated by use of composites for bone tissue restoration prepared by AM. Only composite structures directly fabricated by AM will be discussed and no indirectly fabricated composites using molds prepared by AM.

\subsection{Effect of particle content on processing conditions and properties of composite structures}

As particle content will affect the properties of the source material for fabrication as well as the properties of the fabricated structures, a significant amount of reports focus on elucidating the effect of the particle content and on methods to optimize processing conditions based on particle content.

Tan et al. used SLS on mixtures of polyetheretherketone (PEEK) and HA powders to prepare composite structures[75]. The fabrication process was optimized by adjusting the temperature of the feed powder to just below the glass transition temperature of PEEK. Additionally, the laser energy was adjusted in order to prepare accurate structures without delamination of fabricated layers. High HA loading up to $40 \mathrm{wt}$ \% was possible while still achieving fabrication of structures with high integrity and the desired shape. Also composites of PVA with up to 30 wt.\% HA and HDPE with up to 40 wt.\% HA have been successfully prepared by SLS[43, 76]. These structures could be stably produced by adjustment of the laser power and scan rate. In a later report Wiria et al. used SLS to prepare composite structures[77]. These structures were composed of PCL with up to 30 wt.\% HA. Laser scan rate as well as power was adjusted in order to fabricate stable structures. Structures with 10 wt.\% HA were shown to support pre-osteoblast cell proliferation. In a more recent report, Eshraghi et al. prepared composites of PCL and HA with varying HA content. By adjustment of only the laser power it was possible to fabricate parts with high integrity and up to $30 \mathrm{wt} . \% \mathrm{HA}$.

Elomaa et al. prepared porous composite structures by stereolithography with up to 20 wt.\% of bioactive glass particles in PCL-dimethacrylate networks[55]. Without adjusting any processing parameters it was shown that incorporation of increasing amounts of bioactive glass would decrease the pore size and porosity of the scaffolds, even though the same scaffold design was used. However, the compressive modulus of the scaffolds as well as proliferation of human gingival fibroblasts was increased with increasing particle 
content. Tanodekaew et al. investigated the effect of HA content in composite scaffolds with PDLLA-dimethacrylate networks prepared by stereolithography on pre-osteoblast cell culture[78]. Cells seeded on scaffolds with a higher HA content showed higher ALP activity after 7 days of culture. After 21 days of cell culture, no significant differences in cell proliferation and differentiation was detected however. In recent work describing the fabrication of composite structures of poly(trimethylene carbonate) networks including 0 to 40 wt.\% nano-HA, it was also shown that the nano-HA content did not significantly affect BMSC attachment and proliferation[79]. In a follow-up report it was shown that these structures became enriched with nano-HA at their surface as the particle content is increased. This did result in stronger differentiation of BMSCs into the osteogenic lineage and in a more effective restoration of rabbit calvarial defects after implantation[80]. A significant improvement of osseointegration of the implants was observed due to the surface-enrichment by nano-HA as the calcium phosphate content was increased.

Using composite structures of poly(L-lactic acid) (PLLA) with nano-HA contents between 0 and $40 \mathrm{wt} . \%$, it was shown that the optimal composition of a composite is not necessarily the composition with the highest HA content[81]. When fabricated by LDM it was found that at $20 \mathrm{wt} . \%$ nano-hydroxyapatite, the flexural mechanical properties and porosity and pore size were all enhanced compared to composite structures with lower or higher nanoHA contents. This resulted in better osteoblast adhesion and proliferation. On composites of poly(3-hydroxybutyrate-co-3-hydroxyhexanoate) (PHBHHx) with 25 to $87.5 \mathrm{wt} \%$ bioactive glass it was shown that low bioactive glass contents are actually beneficial[82]. On these composites, bone marrow mesenchymal stem cells (BMSCs) proliferated and differentiated better at low bioactive glass contents. This was ascribed to the presence of a thin PHBHHx film on the bioactive glass particles at low particle contents which enhanced hydrophobicity of the scaffolds. Additionally, the degradation products (3-hydroxybutyrate specifically) may promote cell proliferation and osteogenic differentiation. In vivo, the PHBHHx composites with 25 wt.\% bioactive glass were able to partly restore critical sized defects in rat calvaria.

Yeo et al. investigated the effect of increasing amounts of TCP in PCL/collagen fiber scaffolds prepared by FDM[83]. It was shown that increasing the amount of TCP in the extruded slurry resulted in slimmer lines being deposited due to reduction of the die-swell effect generally associated with extrusion of polymer melts. Increasing amounts of TCP therefore resulted in a higher resolution of the print process although struts were not always homogeneous due to particle agglomeration. Kutikov et al. prepared scaffolds with shape memory of a temperature responsive block-copolymer, PDLLA-block-poly(ethylene glycol)-block-PDLLA (PELA) and prepared composites of it with HA[84]. These composites had significantly improved mechanical properties when 20 wt.\% HA was incorporated and the shape memory capabilities were similar to that of the pure block copolymer. In a follow-up report Kutikov et al. utilized these composites in cell culture 
experiments and showed that fibroblasts and BMSCs would not attach well to PELA scaffolds, but showed good cell attachment and proliferation on the PELA/HA scaffolds[85]. Additionally, differentiation of BMSCs towards the osteogenic lineage was possible.

\subsection{Effect of particle size on processing conditions and properties of composite structures}

Apart from particle content, the effect of particle size in the source material for additive manufacturing of composites has been investigated for some additive manufacturing techniques.

For SLS, it was shown that powder mixtures need similar sized polymer particles and inorganic particles in order to fabricate stable and accurate composite structures[86, 87]. Especially when small inorganic particles compared to the polymer particles are used, accurate fabrication of structures by SLS is not possible. In this case the inorganic particles can cover the polymer particles, preventing the formation of a sintered structure[86]. Eosoly et al. furthermore showed that, next to laser power and distance between traced lines, part orientation should be considered in order to prepare structures with accuracy[88]. Porous composites of PCL with 30 wt.\% HA were prepared and it was shown that structures were most accurately built in the Z-direction. In the XY-direction deviations of the pore morphology were observed due to over-sintering at the edge of the laser beam.

Inzana et al. prepared composites of collagen and HA/tricalcium phosphate(TCP) by 3Dprinting[89]. It was shown that also in this technique, particle size and homogeneity is of importance for accurate scaffold fabrication. Inzana et al. accurately reproduced the femoral mid diaphysis of mice which allowed for the bone regeneration of the femoral shaft in in vivo experiments.

\subsection{Further optimization of fabrication accuracy and properties of composite structures}

Fabrication accuracy of composite scaffolds depends not only on the particle size and content, but also on the homogeneity of the feed material applied in the process and on possible post-treatment of a fabricated part. Some methods to ensure accurate AM fabrication of composite structures are discussed here.

Popov et al. prepared structures in stereolithography using composite resins of oligocarbonate-dimethacrylates with hydroxyapatite (HA) particles[90]. In order to ensure homogeneity of fabricated structures Popov et al. delayed HA aggregation in the resin by grafting poly(acrylic acid) to the particle surface. This strongly reduced the aggregation rate of the particles and therefore allowed for uniform structures to be prepared. Farkas et al. investigated a method to improve the stability of stereolithography resins containing HA particles without grafting any stabilizers to the particles. The HA particles were dispersed in ethanol by laser ablation and poly(propylene fumarate) (PPF) and diethyl fumarate was added to prepare a resin[91]. This resin yielded structures with a homogeneous distribution 
of HA throughout, for particle contents of 50 to $300 \mathrm{ppm}$. Ronca et al. showed the need to adjust essential processing parameters such as resin viscosity, irradiation time and light intensity in stereolithography to prepare composite scaffolds of poly(D,L-lactic acid) (PDLLA) networks with nano-HA contents of up to $14 \mathrm{wt} \%$ [56]. Adjustments of these parameters allowed for the fabrication of structures comprised of only $25 \mu \mathrm{m}$ thick layers.

Furthermore, the post-treatment of as-fabricated structures may play an important role in the preparation of well-defined structures. Martínez-Vázquez et al. showed that the drying process employed to fabricate composites of glutaraldehyde-crosslinked gelatin and silicone-doped hydroxyapatite plays a significant role for the eventual structure that is obtained after LDM fabrication[92]. A significantly more open porosity and a betterdefined structure was obtained by freeze drying these composites compared to drying at 37 ${ }^{\circ} \mathrm{C}$ and ambient pressure. Given the large pore size (in the range of hundreds of micrometers) this did not significantly affect the proliferation and activity of osteoblast cells though.

\subsection{Effects of pore size, porosity and pore geometry of composite structures prepared by AM on their in vitro and in vivo performance}

Given the ability to prepare structures with a pre-defined porosity, pore size and geometry with high accuracy, AM techniques are ideal to investigate the effect of such scaffold design factors on structure properties and osteogenesis. Several researchers have applied different AM techniques for this purpose.

Using poly(D,L-Lactic Acid) (PDLLA) and hydroxyapatite particles (HA), it was shown that structures with very large pore sizes $(1500 \mu \mathrm{m})$ reduce the early differentiation of preosteoblasts [78]. However, after prolonged cell culturing no significant differences in osteogenic differentiation could be detected for scaffolds with very large and smaller (1000 $\mu \mathrm{m})$ pores. The effect of porosity has also been investigated using composites of polypropylene and tricalcium phosphate prepared by FDM[93]. It was shown that the porosity reduced the proliferation of osteoblast cells in vitro.

Pore geometry may affect cell adhesion and proliferation in three dimensional structures and has therefore been investigated in several reports. Korpela et al. prepared composites of PCL and bioactive glass with pore sizes of $400 \mu \mathrm{m}$ and porosity between 30-40\% using FDM. The geometry of the pores was adjusted by a change of the lay-down direction of composite strands in subsequently fabricated layers. It was shown that pore geometry of the composites did not significantly affect fibroblast proliferation[94]. In a report by Channasanon et al. composite implants of PDLLA-dimethacrylate networks and $55 \mathrm{wt} \%$ HA were prepared by stereolithography. The composites were prepared in a woodpile structure and the displacement of each layer in the woodpile was used to modulate the compressive mechanical properties of the structure[95]. It was found that this allows for adjustment of the compressive strength of the structure, whereas the compressive modulus 
was not significantly affected. Kim et al. showed that the use of a displacement between layers of a woodpile structure allowed for good cell adhesion and proliferation using composites of 53 wt.\% PCL, 35 wt.\% poly(lactic-co-glycolic acid) (PLGA) and 12 wt.\% TCP[96].

Apart from a designed porosity at a size scale of several hundred micrometers (i.e. 'macroporosity'), a micro-porosity in the struts of scaffolds is beneficial for bone regeneration[5, 6 , 97]. In this case the macro-porosity allows for the ingrowth of de novo bone and neovascularization, whereas the presence of a micro-porosity allows for prolonged nutritional supply throughout an implanted structure. An additional benefit of the microporosity is an enhanced surface roughness and an increased protein adsorption, which can be beneficial in bone restoration $[5,98,99]$. The LDM technique is often applied to prepare micro-porous scaffolds. In the earliest report on the application of LDM for fabrication of micro-porous composite scaffolds, Xiong et al. prepared composites of PLLA and TCP[34]. Scaffolds with macro-pores of $400 \mu \mathrm{m}$ and micro-pores of an average of $5 \mu \mathrm{m}$ were prepared. Implantation of the scaffolds in canine radial bone defects together with bone morphogenetic protein resulted in fully repaired defects within 24 weeks after implantation. Histological analysis indicated the formation of bone and bone marrow-like tissue in the defects. Liu et al. prepared micro-porous scaffolds of PLLA, PDLLA or poly(lactic-co-glycolic acid) (PLGA) with varying amounts of TCP[35]. Liu et al. showed that mechanical properties were ideal for an intermediate TCP content. In another report, Liu et al. prepared a porous gradient structure in composites of PLGA and $50 \mathrm{wt} \%$ TCP by LDM for use in osteochondral defect restoration[100]. The structure consisted of two parts with a combined micro- and macro-porosity which were separated by a layer with only micro-pores. The micro-porous layer between the micro-/macro-porous sections of the structure would mimic the natural osteochondral tissue, consisting of osteochondral bone and articular cartilage, separated by a dense layer of calcified cartilage. After seeding with BMSCs, the gradient structures were implanted in full osteochondral defects in rabbits and the separate formation of bone and cartilage was observed on either side of the microporous layer in the implanted structure. Serra et al. reported on micro-porous, composite scaffolds of PLA with PEG and bioactive glass prepared by LDM[36]. The amount of PEG was initially varied in the scaffolds, resulting in a coarser surface structure, higher wettability, faster degradation and lower stiffness when the amount of PEG was increased. It was concluded that a moderate amount of PEG (2.5 wt.\%) would yield a scaffold with suitable surface roughness, hydrophilicity and mechanical properties for bone tissue engineering. In a follow-up report, these composite scaffolds were used in cell culture experiments[101]. The prepared scaffolds allowed for attachment and proliferation of rat mesenchymal stem cells and these cells attained a spread morphology on the structures. Duan et al. showed that micro-porous structures of poly(hydroxybutyrate-cohydroxyvalerate) with TCP or PLLA with HA supported the osteogenic activity of osteoblast-like cells[41]. 


\subsection{Further enhancing the properties of composite structures prepared by AM}

Although composite scaffolds are specifically designed to improve bone growth in defects and to allow for bone growth along its surface, several improvements have been made over the years.

Application of oxygen plasma treatment to increase the surface hydrophilicity of implants is commonly applied to increase the biological performance of polymer-based materials. Roh et al. have applied it to composite structures of PCL and nano-HA prepared by FDM[102]. The oxygen plasma treatment increases the proliferation of pre-osteoblast cells, although alkaline phosphatase (ALP) activity was not significantly increased when comparing plasma treated scaffolds with non-treated ones. In a later study, PLGA composite scaffolds with nano-HA as well as TCP were used[103]. An oxygen plasma treatment increased surface roughness and wettability of the scaffolds, due to calcium phosphate enrichment on the scaffold surface by polymer etching. Pre-osteoblast adhesion and proliferation was shown to be improved on these scaffolds. Also in this case, ALP activity of cells on the composite scaffolds was not significantly increased due to plasma treatment. Mineralization on the scaffolds was however significantly increased and the expression of osteocalcin and RunX2 was upregulated. Similarly, PCL and TCP composite structures have been treated with sodium hydroxide solution in several reports in order to enhance the surface roughness and hydrophilicity of the structures[73, 104-108].

Pang et al. optimized micro-porous composite structures of PLGA and $\beta$-TCP by applying a coating of a composite collagen type I sponge containing apatite particles[109]. In cell culture experiments, more BMSCs would adhere to the coated structures compared to uncoated structures. The ALP expression of BMSCs on the coated composites furthermore increased to a greater extent. In a follow-up study, in vivo experiments were performed using the coated and uncoated structures[110]. Over a period of 36 weeks, the scaffolds were evaluated after implantation together with BMSCs in the radial bones of rabbits. Full repair of the defect using the scaffolds with coating was observed and dense lamellar bone with a normal marrow cavity was formed. However, defects could not be fully repaired when using scaffolds without coating. In further research from the same group it was shown that the collagen coating could also be loaded with osteogenically differentiated adipose-derived stem cells or BMSCs in order to enhance the bone regenerating capacity of the composite structures[111-115]. These structures were implanted intramuscular, subcutaneous, interlumbar or in radial bone in small animals. In all cases de novo bone formation was observed and the bone formation was significantly enhanced compared to implants with non-loaded collagen coating. In a work describing the fabrication of PCL composites with $20 \mathrm{wt} \%$ TCP, porous structures were fabricated by FDM and enhanced by wrapping them in BMSC cell sheets[104]. These structures were implanted subcutaneously in mice and compared to structures without cell sheets. Formation of vascularized cancellous bone was observed in structures with cell sheets, whereas only fibrous tissue was formed in the structures without. In a later work, composites of PCL, poly(L-lactic-co- 
D,L- lactic acid) and TCP were prepared and loaded with a hyaluronan gel containing either BMP-2 or osteoblast cells[107]. These were implanted in arterio-venous loops in rats to investigate the vascularization potential. In the osteoblast-loaded structures, vascularization extended into the scaffolds whereas few blood vessels were observed to grow into the BMP-2 loaded structures. In a following report, PCL and TCP structures were loaded with BMP-2 or mesenchymal stem cells and implanted in a segmental defect in tibia of sheep[108]. In the BMP-2-loaded structures significantly more bone formation was observed than in the stem cell-loaded ones.

Some reports discuss the modification of composite structures with anti-microbial agents in order prepare implant structures that may prevent infection following implantation. PLGA/TCP structures were enhanced by the incorporation of magnesium particles[116]. The structures were effective in inhibiting Staphylococcus Aureus growth and biofilm formation. However, cell growth inhibition of pre-osteoblasts was observed at high magnesium content (15 wt.\%). A magnesium content of $10 \mathrm{wt} \%$ was determined to be optimal for bacterial growth inhibition and good biocompatibility of the structures. Another type of anti-microbial implant structure was prepared by Correia et al. using LDM[117]. These structures consisted of calcium-crosslinked alginate with TCP particles which were loaded with silver nitrate $\left(\mathrm{AgNO}_{3}\right)$ nanoparticles by direct incorporation in the feed material for LDM. These structures were non-cytotoxic, supported mineralization by osteoblast cells and were able to inhibit Staphylococcus Aureus growth.

The release of osteopromotive or -genic components from implants is another vastly utilized method to enhance their regenerative capacity. Ma et al. loaded composites of PLGA and TCP prepared by LDM with bovine bone proteins using a dip-coating technique[118]. These composites were thereafter applied in lumbar fusion in rabbits and performed significantly better than composites without bovine bone proteins. Similarly, Abbah et al. coated composite structures of PCL and TCP with collagen and loaded them with BMP-2[106]. These were applied in spinal fusion in pigs. Formation of mature trabecular bone between vertebrae and good integration with the surrounding bone was observed.

A great deal of research has been performed on the release of osteopromotive icaritin and icariin from composite scaffolds of PLGA and TCP. Using LDM, these osteopromotive compounds were directly processed with the composite to form micro-porous structures with the osteopromotive compound dispersed inside. In one particular work, structures loaded with icaritin were compared to structures loaded with BMP-2[119]. The incorporation of icaritin in the composite resulted in a lower degradation rate, retardation of $\mathrm{Ca}^{2+}$ release and improved mechanical properties whereas no significant changes were found from the loading of BMP-2 in the composites. Furthermore, icaritin incorporation resulted in a significantly more potent osteogenic differentiation of BMSCs than BMP-2 incorporation. The icaritin-loaded composite scaffolds were implanted intramuscularly in 
rabbits and in rabbit femora in subsequent reports in order to assess the bone healing and angiogenic capacities of the loaded composite[120, 121]. Implantation of the icaritinloaded composites resulted in both an enhanced bone regeneration as well as neovascularization compared to composites without icaritin[120]. Structures of PLGA and TCP with icaritin were also used to prevent joint collapse in emus with steroid-associated osteonecrosis[122]. The icaritin-loaded composite structures were implanted in osteonecrotic femoral heads of emus and reduced hip collapse incidence compared to structures without icaritin. Icaritin-loaded structures furthermore resulted in more bone formation and higher cartilage thickness and stiffness than the composite structures without icaritin.

Adjustment of the inorganic phase in composites has also been exploited to further improve their efficacy. Zhang et al. investigated the effect of substituting calcium in bioactive glass with strontium in composites with PVA[123]. In this work, the bioactive glass content was kept constant at $50 \mathrm{wt} . \%$. The strontium content in the bioactive glass was varied between 0 and $7 \mathrm{~mol} \%$. On the composite structures, the osteogenic functioning and proliferation of osteoblast-like cells was enhanced when strontium was added to the HA particles. There were no significant differences between composite structures with bioactive glass particles with 2 to $7 \mathrm{~mol} \%$ of strontium however. Also Poh et al. investigated the effect of substituting calcium in bioactive glass with strontium on the differentiation of preosteoblasts[74]. On the structures containing the bioactive glass with strontium, earlier differentiation was observed compared to composites with unmodified bioactive glass. No significant differences in osteogenic differentiation were found during prolonged cell culture however. In a later study, composites of PCL with bioactive glass or the strontium containing bioactive glass were implanted subcutaneously in mice[67]. In both types of composite structures, fibrous and adipose tissue was observed and no formation of mature bone. Mineralization islets were found in the tissues in composites with the strontium containing bioactive glass however.

\subsection{Specifically shaped implant structures}

A major advantage of the ability to prepare accurately defined shapes is the possibility to fabricate implant structures based on specific bone defects. Especially for complex or craniomaxillofacial fractures this is a valuable asset. Nonetheless, few reports in which AM has been applied to prepare composite structures that accurately fit bony defects are published to date. Probst et al. applied FDM in the fabrication of composite scaffolds of PCL and TCP for calvarial defects[124]. A structure designed according to CT images of a human calvarial defect was prepared. Implantation of the structure into the defect resulted in seamless integration and osseous merger was detected after 6 months. Corcione et al. demonstrated the feasibility of preparing porous maxillary sinus structures using filaments of PLA with HA, processed by FDM[125]. The maxillary sinus model was based on CT imaging data and the composite structure matched the dimensions and shape of the model 
well. In work of our own group, we have shown that composites of poly(trimethylene carbonate) networks and nano-HA in the shape of orbital floor implants can readily be prepared by stereolithography. These composite implant structures were based on CT-scans of patients with blow-out orbital floor fractures and contained up to $40 \mathrm{wt} \%$ of nanoHA[28].

\section{Conclusions}

Several AM techniques have been shown to be suitable for the fabrication of composite structures for bone tissue restoration. Research from the past years has demonstrated an increasing understanding of the adjustments required to accurately fabricate composites. Furthermore, AM of composite structures has led to new insights regarding the reconstruction of bone defects. Additional modifications of composites by surface treatments, coatings, osteopromotive and osteoinductive compounds, anti-microbial agents and addition of a modified inorganic phase are further enhancing their osteogenic efficacy. This offers a promising perspective for further development of implant structures for bone reconstruction and the clinical application of such structures.

\section{References}

1. Do, A.V., B. Khorsand, S.M. Geary, and A.K. Salem, 3D Printing of Scaffolds for Tissue Regeneration Applications. Advanced healthcare materials, 2015. 4(12): p. 1742-1762.

2. Wüst, S., R. Muller, and S. Hofmann, Controlled Positioning of Cells in Biomaterials-Approaches Towards 3D Tissue Printing. Journal of functional biomaterials, 2011. 2(3): p. 119-154.

3. Peltola, S.M., F.P. Melchels, D.W. Grijpma, and M. Kellomaki, A review of rapid prototyping techniques for tissue engineering purposes. Ann Med, 2008. 40(4): p. 268-280.

4. Loh, Q.L. and C. Choong, Three-dimensional scaffolds for tissue engineering applications: role of porosity and pore size. Tissue engineering Part B Reviews, 2013. 19(6): p. 485-502.

5. Bose, S., M. Roy, and A. Bandyopadhyay, Recent advances in bone tissue engineering scaffolds. Trends Biotechnol, 2012. 30(10): p. 546-554.

6. Karageorgiou, V. and D. Kaplan, Porosity of $3 D$ biomaterial scaffolds and osteogenesis. Biomaterials, 2005. 26(27): p. 5474-5491.

7. Roy, T.D., J.L. Simon, J.L. Ricci, E.D. Rekow, V.P. Thompson, and J.R. Parsons, Performance of degradable composite bone repair products made via threedimensional fabrication techniques. Journal of Biomedical Materials Research Part A, 2003. 66(2): p. 283-291.

8. Kruyt, M.C., J.D. de Bruijn, C.E. Wilson, F.C. Oner, C.A. van Blitterswijk, A.J. Verbout, and W.J. Dhert, Viable osteogenic cells are obligatory for tissue- 
engineered ectopic bone formation in goats. Tissue Engineering, 2003. 9(2): p. 327-336.

9. Melchels, F.P., K. Bertoldi, R. Gabbrielli, A.H. Velders, J. Feijen, and D.W. Grijpma, Mathematically defined tissue engineering scaffold architectures prepared by stereolithography. Biomaterials, 2010. 31(27): p. 6909-6916.

10. Wendel, B., D. Rietzel, F. Kuhnlein, R. Feulner, G. Hulder, and E. Schmachtenberg, Additive Processing of Polymers. Macromolecular Materials and Engineering, 2008. 293(10): p. 799-809.

11. Hutmacher, D.W., M. Sittinger, and M.V. Risbud, Scaffold-based tissue engineering: rationale for computer-aided design and solid free-form fabrication systems. Trends Biotechnol, 2004. 22(7): p. 354-362.

12. Lantada, A.D. and P.L. Morgado, Rapid prototyping for biomedical engineering: current capabilities and challenges. Annual review of biomedical engineering, 2012. 14: p. 73-96.

13. Billiet, T., M. Vandenhaute, J. Schelfhout, S. Van Vlierberghe, and P. Dubruel, A review of trends and limitations in hydrogel-rapid prototyping for tissue engineering. Biomaterials, 2012. 33(26): p. 6020-6041.

14. Seol, Y.J., T.Y. Kang, and D.W. Cho, Solid freeform fabrication technology applied to tissue engineering with various biomaterials. Soft Matter, 2012. 8(6): p. 1730-1735.

15. Hutmacher, D.W. and S. Cool, Concepts of scaffold-based tissue engineering--the rationale to use solid free-form fabrication techniques. Journal of Cellular \& Molecular Medicine, 2007. 11(4): p. 654-669.

16. Hutmacher, D.W., J.T. Schantz, C.X. Lam, K.C. Tan, and T.C. Lim, State of the art and future directions of scaffold-based bone engineering from a biomaterials perspective. Journal of Tissue Engineering and Regenerative Medicine, 2007. 1(4): p. 245-260.

17. Moroni, L., A. Nandakumar, F.B. de Groot, C.A. van Blitterswijk, and P. Habibovic, Plug and play: combining materials and technologies to improve bone regenerative strategies. Journal of Tissue Engineering \& Regenerative Medicine, 2015. 9(7): p. 745-759.

18. Hinderer, S., S.L. Layland, and K. Schenke-Layland, ECM and ECM-like materials - Biomaterials for applications in regenerative medicine and cancer therapy. Adv Drug Deliv Rev, 2016. 97: p. 260-269.

19. Izadifar, M., A. Haddadi, X. Chen, and M.E. Kelly, Rate-programming of nanoparticulate delivery systems for smart bioactive scaffolds in tissue engineering. Nanotechnology, 2015. 26(1): p. 012001.

20. Slepicka, P., N.S. Kasalkova, J. Siegel, Z. Kolska, L. Bacakova, and V. Svorcik, Nano-structured and functionalized surfaces for cytocompatibility improvement and bactericidal action. Biotechnol Adv, 2015. 33(6 Pt 2): p. 1120-1129. 
21. Rashidi, H., J. Yang, and K.M. Shakesheff, Surface engineering of synthetic polymer materials for tissue engineering and regenerative medicine applications. Biomaterials Science, 2014. 2(10): p. 1318-1331.

22. Pfister, A., R. Landers, A. Laib, U. Hubner, R. Schmelzeisen, and R. Mulhaupt, Biofunctional rapid prototyping for tissue-engineering applications: $3 D$ bioplotting versus $3 D$ printing. Journal of Polymer Science Part A - Polymer Chemistry, 2004. 42(3): p. 624-638.

23. Bertsch, A., S. Jiguet, P. Bernhard, and P. Renaud, Microstereolithography: $a$ review. Rapid Prototyping Technologies, 2003. 758: p. 3-15.

24. Bertsch, A. and P. Renoud, Microstereolithography, in Stereolithography, P.J. Bártolo, Editor. 2011, Springer Science + Business Media: New York. p. 81-112.

25. Maruo, S. and K. Ikuta, Submicron stereolithography for the production of freely movable mechanisms by using single-photon polymerization. Sensors and Actuators A -Physical, 2002. 100(1): p. 70-76.

26. Tumbleston, J.R., D. Shirvanyants, N. Ermoshkin, R. Janusziewicz, A.R. Johnson, D. Kelly, K. Chen, R. Pinschmidt, J.P. Rolland, A. Ermoshkin, E.T. Samulski, and J.M. DeSimone, Additive manufacturing. Continuous liquid interface production of 3 D objects. Science, 2015. 347(6228): p. 1349-1352.

27. Hinczewski, C., S. Corbel, and T. Chartier, Ceramic suspensions suitable for stereolithography. Journal of the European Ceramic Society, 1998. 18(6): p. 583590.

28. Chapter 4 of this thesis; Geven, M.A., V. Varjas, L. Kamer, X.J. Wang, J. Peng, D. Eglin, and D.W. Grijpma, Fabrication of patient specific composite orbital floor implants by stereolithography. Polymers for Advanced Technologies, 2015. 26(12): p. 1433-1438.

29. Lee, C.S., S.G. Kim, H.J. Kim, and S.H. Ahn, Measurement of anisotropic compressive strength of rapid prototyping parts. Journal of Materials Processing Technology, 2007. 187: p. 627-630.

30. Ahn, S.H., M. Montero, D. Odell, S. Roundy, and P.K. Wright, Anisotropic material properties of fused deposition modeling ABS. Rapid Prototyping Journal, 2002. 8(4): p. 248-257.

31. Nikzad, M., S.H. Masood, and I. Sbarski, Thermo-mechanical properties of a highly filled polymeric composites for Fused Deposition Modeling. Materials \& Design, 2011. 32(6): p. 3448-3456.

32. Kumar, S. and J.P. Kruth, Composites by rapid prototyping technology. Materials \& Design, 2010. 31(2): p. 850-856.

33. Wang, F., L. Shor, A. Darling, S. Khalil, W. Sun, S. Guceri, and A. Lau, Precision extruding deposition and characterization of cellular poly-epsilon-caprolactone tissue scaffolds. Rapid Prototyping Journal, 2004. 10(1): p. 42-49. 
34. Xiong, Z., Y.N. Yan, S.G. Wang, R.J. Zhang, and C. Zhang, Fabrication of porous scaffolds for bone tissue engineering via low-temperature deposition. Scripta Materialia, 2002. 46(11): p. 771-776.

35. Liu, L., Z. Xiong, Y. Yan, Y. Hu, R. Zhang, and S. Wang, Porous morphology, porosity, mechanical properties of poly(alpha-hydroxy acid)-tricalcium phosphate composite scaffolds fabricated by low-temperature deposition. Journal of Biomedical Materials Research A, 2007. 82(3): p. 618-629.

36. Serra, T., M. Ortiz-Hernandez, E. Engel, J.A. Planell, and M. Navarro, Relevance of PEG in PLA-based blends for tissue engineering 3D-printed scaffolds. Materials Science \& Engineering Part C - Materials for Biological Applications, 2014. 38: p. 55-62.

37. Landers, R. and R. Mulhaupt, Desktop manufacturing of complex objects, prototypes and biomedical scaffolds by means of computer-assisted design combined with computer-guided 3D plotting of polymers and reactive oligomers. Macromolecular Materials and Engineering, 2000. 282(9): p. 17-21.

38. Mueller, S., E.W. Llewellin, and H.M. Mader, The rheology of suspensions of solid particles. Proceedings of the Royal Society A - Mathematical, Physical and Engineering Sciences, 2010. 466(2116): p. 1201-1228.

39. Li, M.G., X.Y. Tian, and X.B. Chen, A brief review of dispensing-based rapid prototyping techniques in tissue scaffold fabrication: role of modeling on scaffold properties prediction. Biofabrication, 2009. 1(3): p. 032001.

40. Woodfield, T.B., J. Malda, J. de Wijn, F. Peters, J. Riesle, and C.A. van Blitterswijk, Design of porous scaffolds for cartilage tissue engineering using a three-dimensional fiber-deposition technique. Biomaterials, 2004. 25(18): p. 41494161.

41. Duan, B., M. Wang, W.Y. Zhou, W.L. Cheung, Z.Y. Li, and W.W. Lu, Threedimensional nanocomposite scaffolds fabricated via selective laser sintering for bone tissue engineering. Acta Biomater, 2010. 6(12): p. 4495-4505.

42. Zhou, W.Y., S.H. Lee, M. Wang, W.L. Cheung, and W.Y. Ip, Selective laser sintering of porous tissue engineering scaffolds from poly(L)/carbonated hydroxyapatite nanocomposite microspheres. Journal of Materials ScienceMaterials in Medicine, 2008. 19(7): p. 2535-2540.

43. Hao, L., M.M. Savalani, Y. Zhang, K.E. Tanner, and R.A. Harris, Selective laser sintering of hydroxyapatite reinforced polyethylene composites for bioactive implants and tissue scaffold development. Proceedings of the Institution of Mechanical Engineers Part H, 2006. 220(4): p. 521-531.

44. Starly, B., W. Lau, T. Bradbury, and W. Sun, Internal architecture design and freeform fabrication of tissue replacement structures. Computer-Aided Design, 2006. 38(2): p. 115-124.

45. Starly, B. and W. Sun, Internal Scaffold Architecture Designs using Lindenmayer Systems. Computer-Aided Design \& Applications, 2007. 4(1-4): p. 395-403. 
46. Rajagopalan, S. and R.A. Robb, Schwarz meets Schwann: design and fabrication of biomorphic and durataxic tissue engineering scaffolds. Med Image Anal, 2006. 10(5): p. 693-712.

47. Giannitelli, S.M., D. Accoto, M. Trombetta, and A. Rainer, Current trends in the design of scaffolds for computer-aided tissue engineering. Acta Biomater, 2014. 10(2): p. 580-594.

48. Yoo, D.J., Computer-aided Porous Scaffold Design for Tissue Engineering Using Triply Periodic Minimal Surfaces. International Journal of Precision Engineering and Manufacturing, 2011. 12(1): p. 61-71.

49. Yoo, D.J., New paradigms in internal architecture design and freeform fabrication of tissue engineering porous scaffolds. Med Eng Phys, 2012. 34(6): p. 762-776.

50. Narra, N., S.B. Blanquer, S.P. Haimi, D.W. Grijpma, and J. Hyttinen, mCT based assessment of mechanical deformation of designed PTMC scaffolds. Clinical Hemorheology \& Microcirculation, 2015. 60(1): p. 99-108.

51. Blanquer, S.B.G., M. Werner, M. Hannula, S. Sharifi, G.P.R. Lajoinie, D. Eglin, J. Hyttinen, A.A. Poot, and D.W. Grijpma, Surface curvature in triply-periodic minimal surface architectures as a distinct design parameter in preparing advanced tissue engineering scaffolds. Biofabrication, 2017. 9(2): p. 025001.

52. Melchels, F.P., B. Tonnarelli, A.L. Olivares, I. Martin, D. Lacroix, J. Feijen, D.J. Wendt, and D.W. Grijpma, The influence of the scaffold design on the distribution of adhering cells after perfusion cell seeding. Biomaterials, 2011. 32(11): p. 28782884.

53. Melchels, F.P., J. Feijen, and D.W. Grijpma, A poly(D,L-lactide) resin for the preparation of tissue engineering scaffolds by stereolithography. Biomaterials, 2009. 30(23-24): p. 3801-3809.

54. Melchels, F.P., A.M. Barradas, C.A. van Blitterswijk, J. de Boer, J. Feijen, and D.W. Grijpma, Effects of the architecture of tissue engineering scaffolds on cell seeding and culturing. Acta Biomater, 2010. 6(11): p. 4208-17.

55. Elomaa, L., A. Kokkari, T. Narhi, and J.V. Seppala, Porous 3D modeled scaffolds of bioactive glass and photocrosslinkable poly(epsilon-caprolactone) by stereolithography. Composites Science and Technology, 2013. 74: p. 99-106.

56. Ronca, A., L. Ambrosio, and D.W. Grijpma, Preparation of designed poly $(D, L-$ lactide)/nanosized hydroxyapatite composite structures by stereolithography. Acta Biomater, 2013. 9(4): p. 5989-5996.

57. Seck, T.M., F.P. Melchels, J. Feijen, and D.W. Grijpma, Designed biodegradable hydrogel structures prepared by stereolithography using poly(ethylene glycol)/poly(D,L-lactide)-based resins. Journal of Controlled Release, 2010. 148(1): p. 34-41.

58. Schuller-Ravoo, S., S.M. Teixeira, J. Feijen, D.W. Grijpma, and A.A. Poot, Flexible and elastic scaffolds for cartilage tissue engineering prepared by 
stereolithography using poly(trimethylene carbonate)-based resins. Macromolecular bioscience, 2013. 13(12): p. 1711-1719.

59. Jansen, J., F.P. Melchels, D.W. Grijpma, and J. Feijen, Fumaric acid monoethyl ester-functionalized poly(D,L-lactide)/N-vinyl-2-pyrrolidone resins for the preparation of tissue engineering scaffolds by stereolithography. Biomacromolecules, 2009. 10(2): p. 214-220.

60. Blanquer, S.B., S. Sharifi, and D.W. Grijpma, Development of poly(trimethylene carbonate) network implants for annulus fibrosus tissue engineering. Journal of Applied Biomaterials \& Functional Materials, 2012. 10(3): p. 177-184.

61. Blanquer, S.B.G., S.P. Haimi, A.A. Poot, and D.W. Grijpma, Effect of Pore Characteristics on Mechanical Properties and Annulus Fibrosus Cell Seeding and Proliferation in Designed PTMC Tissue Engineering Scaffolds. Macromolecular Symposia, 2013. 334(1): p. 75-81.

62. van Bochove, B., G. Hannink, P. Buma, and D.W. Grijpma, Preparation of Designed Poly(trimethylene carbonate) Meniscus Implants by Stereolithography: Challenges in Stereolithography. Macromolecular bioscience, 2016. 16(12): p. 1853-1863.

63. Przeradzka, M.A., B. van Bochove, T.C. Bor, and D.W. Grijpma, Phase-separated mixed-macromer hydrogel networks and scaffolds prepared by stereolithography. Polymers for Advanced Technologies, 2017. 28(10): p. 1212-1218.

64. Sharifi, S., S. Blanquer, and D.W. Grijpma, Polymeric microstructures with shape-memory properties for biomedical use built by stereolithography. Journal of Applied Biomaterials \& Functional Materials, 2012. 10(3): p. 280-286.

65. Fu, Q., E. Saiz, M.N. Rahaman, and A.P. Tomsia, Toward Strong and Tough Glass and Ceramic Scaffolds for Bone Repair. Advanced Functional Materials, 2013. 23(44): p. 5461-5476.

66. Chim, H., D.W. Hutmacher, A.M. Chou, A.L. Oliveira, R.L. Reis, T.C. Lim, and J.T. Schantz, A comparative analysis of scaffold material modifications for loadbearing applications in bone tissue engineering. Interational Journal of Oral \& Maxillofacial Surgery, 2006. 35(10): p. 928-934.

67. Poh, P.S.P., D.W. Hutmacher, B.M. Holzapfel, A.K. Solanki, M.M. Stevens, and M.A. Woodruff, In vitro and in vivo bone formation potential of surface calcium phosphate-coated polycaprolactone and polycaprolactone/bioactive glass composite scaffolds. Acta Biomater, 2016. 30: p. 319-333.

68. Hasegawa, S., M. Neo, J. Tamura, S. Fujibayashi, M. Takemoto, Y. Shikinami, K. Okazaki, and T. Nakamura, In vivo evaluation of a porous hydroxyapatite/polyDL-lactide composite for bone tissue engineering. Journal of Biomedical Materials Research Part A, 2007. 81(4): p. 930-938.

69. Wei, G. and P.X. Ma, Structure and properties of nano-hydroxyapatite/polymer composite scaffolds for bone tissue engineering. Biomaterials, 2004. 25(19): p. 4749-4757. 
70. Chatterjee, K., S. Lin-Gibson, W.E. Wallace, S.H. Parekh, Y.J. Lee, M.T. Cicerone, M.F. Young, and C.G. Simon, Jr., The effect of 3D hydrogel scaffold modulus on osteoblast differentiation and mineralization revealed by combinatorial screening. Biomaterials, 2010. 31(19): p. 5051-5062.

71. Nam, J., J. Johnson, J.J. Lannutti, and S. Agarwal, Modulation of embryonic mesenchymal progenitor cell differentiation via control over pure mechanical modulus in electrospun nanofibers. Acta Biomater, 2011. 7(4): p. 1516-1524.

72. Gomez-Cerezo, N., S. Sanchez-Salcedo, I. Izquierdo-Barba, D. Arcos, and M. Vallet-Regi, In vitro colonization of stratified bioactive scaffolds by pre-osteoblast cells. Acta Biomater, 2016. 44: p. 73-84.

73. Zhou, Y., D.W. Hutmacher, S.-L. Varawan, and T.M. Lim, In vitro bone engineering based on polycaprolactone and polycaprolactone-tricalcium phosphate composites. Polymer International, 2007. 56(3): p. 333-342.

74. Poh, P.S.P., D.W. Hutmacher, M.M. Stevens, and M.A. Woodruff, Fabrication and in vitro characterization of bioactive glass composite scaffolds for bone regeneration. Biofabrication, 2013. 5(4): p. 045005.

75. Tan, K.H., C.K. Chua, K.F. Leong, C.M. Cheah, P. Cheang, M.S. Abu Bakar, and S.W. Cha, Scaffold development using selective laser sintering of polyetheretherketone-hydroxyapatite biocomposite blends. Biomaterials, 2003. 24(18): p. 3115-3123.

76. Chua, C.K., K.F. Leong, K.H. Tan, F.E. Wiria, and C.M. Cheah, Development of tissue scaffolds using selective laser sintering of polyvinyl alcohol/hydroxyapatite biocomposite for craniofacial and joint defects. Journal of Materials Science: Materials in Medicine, 2004. 15(10): p. 1113-1121.

77. Wiria, F.E., K.F. Leong, C.K. Chua, and Y. Liu, Poly-epsiloncaprolactone/hydroxyapatite for tissue engineering scaffold fabrication via selective laser sintering. Acta Biomater, 2007. 3(1): p. 1-12.

78. Tanodekaew, S., S. Channasanon, P. Kaewkong, and P. Uppanan, PLA-HA scaffolds: preparation and bioactivity. 3rd International Conference on Tissue Engineering, 2013. 59: p. 144-149.

79. Chapter 6 of this thesis; Guillaume, O., M.A. Geven, D.W. Grijpma, T.T. Tang, L. Qin, Y.X. Lai, H. Yuan, R.G. Richards, and D. Eglin, Poly(trimethylene carbonate) and nano-hydroxyapatite porous scaffolds manufactured by stereolithography. Polymers for Advanced Technologies, 2017. 28(10): p. 12191225.

80. Chapter 7 of this thesis; Guillaume, O., M.A. Geven, C.M. Sprecher, V.A. Stadelmann, D.W. Grijpma, T.T. Tang, L. Qin, Y. Lai, M. Alini, J.D. de Bruijn, H. Yuan, R.G. Richards, and D. Eglin, Surface-enrichment with hydroxyapatite nanoparticles in stereolithography-fabricated composite polymer scaffolds promotes bone repair. Acta Biomater, 2017. 54: p. 386-398. 
81. Huang, J., J. Xiong, J. Liu, W. Zhu, J. Chen, L. Duan, J. Zhang, and D. Wang, Evaluation of the novel three-dimensional porous poly (L-lactic acid)/nanohydroxyapatite composite scaffold. Biomedical Materials Engineering, 2015. 26 Suppl 1: p. S197-5205.

82. Zhao, S., M. Zhu, J. Zhang, Y. Zhang, Z. Liu, Y. Zhu, and C. Zhang, Three dimensionally printed mesoporous bioactive glass and poly(3-hydroxybutyrate-co3-hydroxyhexanoate) composite scaffolds for bone regeneration. Journal of Materials Chemistry B, 2014. 2(36): p. 6106-6118.

83. Yeo, M., H. Lee, and G. Kim, Three-dimensional hierarchical composite scaffolds consisting of polycaprolactone, beta-tricalcium phosphate, and collagen nanofibers: fabrication, physical properties, and in vitro cell activity for bone tissue regeneration. Biomacromolecules, 2011. 12(2): p. 502-510.

84. Kutikov, A.B., K.A. Reyer, and J. Song, Shape Memory Performance of Thermoplastic Amphiphilic Triblock Copolymer poly(D,L-lactic acid-co-ethylene glycol-co-D,L-lactic acid) (PELA)/Hydroxyapatite Composites. Macromolecular Chemistry \& Physics, 2014. 215(24): p. 2482-2490.

85. Kutikov, A.B., A. Gurijala, and J. Song, Rapid prototyping amphiphilic polymer/hydroxyapatite composite scaffolds with hydration-induced self-fixation behavior. Tissue Engineering Part C Methods, 2015. 21(3): p. 229-241.

86. Simpson, R.L., F.E. Wiria, A.A. Amis, C.K. Chua, K.F. Leong, U.N. Hansen, M. Chandrasekaran, and M.W. Lee, Development of a 95/5 poly(L-lactide-coglycolide)/hydroxylapatite and beta-tricalcium phosphate scaffold as bone replacement material via selective laser sintering. Journal of Biomedical Materials Research Part B Applied Biomaterials, 2008. 84(1): p. 17-25.

87. Wiria, F.E., C.K. Chua, K.F. Leong, Z.Y. Quah, M. Chandrasekaran, and M.W. Lee, Improved biocomposite development of poly(vinyl alcohol) and hydroxyapatite for tissue engineering scaffold fabrication using selective laser sintering. Journal of Materials Science: Materials in Medicine, 2008. 19(3): p. 989-996.

88. Eosoly, S., D. Brabazon, S. Lohfeld, and L. Looney, Selective laser sintering of hydroxyapatite/poly-epsilon-caprolactone scaffolds. Acta Biomater, 2010. 6(7): p. 2511-2517.

89. Inzana, J.A., D. Olvera, S.M. Fuller, J.P. Kelly, O.A. Graeve, E.M. Schwarz, S.L. Kates, and H.A. Awad, 3D printing of composite calcium phosphate and collagen scaffolds for bone regeneration. Biomaterials, 2014. 35(13): p. 4026-4034.

90. Popov, V.K., A.V. Evseev, A.L. Ivanov, V.V. Roginski, A.I. Volozhin, and S.M. Howdle, Laser stereolithography and supercritical fluid processing for customdesigned implant fabrication. Journal of materials science. Materials in medicine, 2004. 15(2): p. 123-128.

91. Farkas, B., M. Rodio, I. Romano, A. Diaspro, R. Intartaglia, and S. Beke, Fabrication of hybrid nanocomposite scaffolds by incorporating ligand-free 
hydroxyapatite nanoparticles into biodegradable polymer scaffolds and release studies. Beilstein Journal of Nanotechnology, 2015. 6: p. 2217-2223.

92. Martínez-Vázquez, F.J., M.V. Cabañas, J.L. Paris, D. Lozano, and M. Vallet-Regí, Fabrication of novel Si-doped hydroxyapatite/gelatine scaffolds by rapid prototyping for drug delivery and bone regeneration. Acta Biomater, 2015. 15: p. 200-209.

93. Kalita, S.J., S. Bose, H.L. Hosick, and A. Bandyopadhyay, Development of controlled porosity polymer-ceramic composite scaffolds via fused deposition modeling. Materials Science \& Engineering C-Biomimetic and Supramolecular Systems, 2003. 23(5): p. 611-620.

94. Korpela, J., A. Kokkari, H. Korhonen, M. Malin, T. Narhi, and J. Seppala, Biodegradable and bioactive porous scaffold structures prepared using fused deposition modeling. Journal Biomedical Materials Research Part B Applied Biomaterials, 2013. 101(4): p. 610-619.

95. Channasanon, S., P. Udomkusonsri, S. Chantaweroad, P. Tesavibul, and S. Tanodekaew, Gentamicin Released from Porous Scaffolds Fabricated by Stereolithography. Journal of Healthcare Engineering, 2017. 2017: p. 9547896.

96. Kim, J.Y., J.J. Yoon, E.K. Park, D.S. Kim, S.Y. Kim, and D.W. Cho, Cell adhesion and proliferation evaluation of SFF-based biodegradable scaffolds fabricated using a multi-head deposition system. Biofabrication, 2009. 1(1): p. 015002.

97. Moroni, L., R. Licht, J. de Boer, J.R. de Wijn, and C.A. van Blitterswijk, Fiber diameter and texture of electrospun PEOT/PBT scaffolds influence human mesenchymal stem cell proliferation and morphology, and the release of incorporated compounds. Biomaterials, 2006. 27(28): p. 4911-4922.

98. Habibovic, P. and K. de Groot, Osteoinductive biomaterials--properties and relevance in bone repair. Journal of Tissue Engineering \& Regenerative Medicine, 2007. 1(1): p. 25-32.

99. Tarafder, S., V.K. Balla, N.M. Davies, A. Bandyopadhyay, and S. Bose, Microwave-sintered 3D printed tricalcium phosphate scaffolds for bone tissue engineering. Journal of Tissue Engineering \& Regenerative Medicine, 2013. 7(8): p. 631-641.

100. Liu, L., Z. Xiong, R. Zhang, L. Jin, and Y. Yan, A Novel Osteochondral Scaffold Fabricated via Multi-nozzle Low-temperature Deposition Manufacturing. Journal of Bioactive and Compatible Polymers, 2009. 24(1_suppl): p. 18-30.

101. Serra, T., J.A. Planell, and M. Navarro, High-resolution PLA-based composite scaffolds via 3-D printing technology. Acta Biomater, 2013. 9(3): p. 5521-5530.

102. Roh, H.S., S.W. Myung, S.-C. Jung, and B.-H. Kim, Fabrication of 3D Scaffolds with Nano-Hydroxyapatite for Improving the Preosteoblast Cell-Biological Performance. Journal of Nanoscience and Nanotechnology, 2015. 15(8): p. 55855588. 
103. Roh, H.-S., S.-C. Jung, M.-S. Kook, and B.-H. Kim, In vitro study of $3 D$ PLGA/nHAp/b-TCP composite scaffolds with etched oxygen plasma surface modification in bone tissue engineering. Applied Surface Science, 2016. 388(Part A): p. 321330 .

104. Zhou, Y., F. Chen, S.T. Ho, M.A. Woodruff, T.M. Lim, and D.W. Hutmacher, Combined marrow stromal cell-sheet techniques and high-strength biodegradable composite scaffolds for engineered functional bone grafts. Biomaterials, 2007. 28(5): p. 814-824.

105. Schantz, J.T., A. Brandwood, D.W. Hutmacher, H.L. Khor, and K. Bittner, Osteogenic differentiation of mesenchymal progenitor cells in computer designed fibrin-polymer-ceramic scaffolds manufactured by fused deposition modeling. Journal of Materials Science: Materials in Medicine, 2005. 16(9): p. 807-819.

106. Abbah, S.A., C.X. Lam, D.W. Hutmacher, J.C. Goh, and H.K. Wong, Biological performance of a polycaprolactone-based scaffold used as fusion cage device in a large animal model of spinal reconstructive surgery. Biomaterials, 2009. 30(28): p. 5086-5093.

107. Rath, S.N., G. Pryymachuk, O.A. Bleiziffer, C.X. Lam, A. Arkudas, S.T. Ho, J.P. Beier, R.E. Horch, D.W. Hutmacher, and U. Kneser, Hyaluronan-based heparinincorporated hydrogels for generation of axially vascularized bioartificial bone tissues: in vitro and in vivo evaluation in a PLDLLA-TCP-PCL-composite system. Journal of Materials Science: Materials in Medicine, 2011. 22(5): p. 1279-1291.

108. Reichert, J.C., A. Cipitria, D.R. Epari, S. Saifzadeh, P. Krishnakanth, A. Berner, M.A. Woodruff, H. Schell, M. Mehta, M.A. Schuetz, G.N. Duda, and D.W. Hutmacher, A tissue engineering solution for segmental defect regeneration in load-bearing long bones. Science Translational Medicine, 2012. 4(141): p. 141 ra93.

109. Pang, L., Y. Hu, Y. Yan, L. Liu, Z. Xiong, Y. Wei, and J. Bai, Surface modification of PLGA/ $\beta$-TCP scaffold for bone tissue engineering: Hybridization with collagen and apatite. Surface and Coatings Technology, 2007. 201(24): p. 9549-9557.

110. Pang, L., W. Hao, M. Jiang, J. Huang, Y. Yan, and Y. Hu, Bony defect repair in rabbit using hybrid rapid prototyping polylactic-co-glycolic acid/betatricalciumphosphate collagen I/apatite scaffold and bone marrow mesenchymal stem cells. Indian Journal of Orthopeadics, 2013. 47(4): p. 388-394.

111. Hao, W., Y.Y. Hu, Y.Y. Wei, L. Pang, R. Lv, J.P. Bai, Z. Xiong, and M. Jiang, Collagen I gel can facilitate homogenous bone formation of adipose-derived stem cells in PLGA-beta-TCP scaffold. Cells Tissues Organs, 2008. 187(2): p. 89-102.

112. Hao, W., L. Pang, M. Jiang, R. Lv, Z. Xiong, and Y.Y. Hu, Skeletal repair in rabbits using a novel biomimetic composite based on adipose-derived stem cells encapsulated in collagen I gel with PLGA-beta-TCP scaffold. Journal of Orthopeadic Research, 2010. 28(2): p. 252-257. 
113. Ma, X., Y.Y. Hu, X.M. Wu, J. Liu, Z. Xiong, Y.N. Yan, R. Lv, and J. Wang, Fabrication of a RP-based Biomimetic Grafting Material for Bone Tissue Engineering. Materials Science Forum, 2009. 626-627: p. 6.

114. Ma, X., X. Wu, Y. Wu, J. Liu, Z. Xiong, R. Lv, Y. Yan, J. Wang, and D. Li, Posterolateral Spinal Fusion in Rabbits Using a RP-based PLGA/ TCP/Col/BMSCs-OB Biomimetic Grafting Material. Journal of Bioactive and Compatible Polymers, 2009. 24(5): p. 457-472.

115. Wang, C., G. Meng, L. Zhang, Z. Xiong, and J. Liu, Physical properties and biocompatibility of a core-sheath structure composite scaffold for bone tissue engineering in vitro. Journal of Biomedicine \& Biotechnology, 2012. 2012: p. 579141.

116. Ma, R., Y.X. Lai, L. Li, H.L. Tan, J.L. Wang, Y. Li, T.T. Tang, and L. Qin, Bacterial inhibition potential of $3 D$ rapid-prototyped magnesium-based porous composite scaffolds--an in vitro efficacy study. Scientific Reports, 2015. 5: p. 13775.

117. Correia, T.R., D.R. Figueira, K.D. de Sa, S.P. Miguel, R.G. Fradique, A.G. Mendonca, and I.J. Correia, 3D Printed scaffolds with bactericidal activity aimed for bone tissue regeneration. International Journal of Biological Macromolecules, 2016. 93(Pt B): p. 1432-1445.

118. Ma, X., Y. Hu, R. Lv, J. Wang, X. Wu, and Y. Yan, Multilevel Posterior Lumbar Interlaminar Fusion in Rabbits Using Bovine Bone Protein Extract Delivered by a $R P$-synthesized $3 D$ Biopolymer Construct. Journal of Bioactive and Compatible Polymers, 2010. 25(5): p. 513-526.

119. Chen, S.H., X.L. Wang, X.H. Xie, L.Z. Zheng, D. Yao, D.P. Wang, Y. Leng, G. Zhang, and L. Qin, Comparative study of osteogenic potential of a composite scaffold incorporating either endogenous bone morphogenetic protein-2 or exogenous phytomolecule icaritin: an in vitro efficacy study. Acta Biomater, 2012. 8(8): p. 3128-3137.

120. Wang, X.L., X.H. Xie, G. Zhang, S.H. Chen, D. Yao, K. He, X.H. Wang, X.S. Yao, Y. Leng, K.P. Fung, K.S. Leung, and L. Qin, Exogenous phytoestrogenic molecule icaritin incorporated into a porous scaffold for enhancing bone defect repair. Journal of Orthopeadic Research, 2013. 31(1): p. 164-172.

121. Xie, X.H., X.L. Wang, G. Zhang, Y.X. He, Y. Leng, T.T. Tang, X. Pan, and L. Qin, Biofabrication of a PLGA-TCP-based porous bioactive bone substitute with sustained release of icaritin. Journal of Tissue Engineering \& Regenerative Medicine, 2015. 9(8): p. 961-972.

122. Qin, L., D. Yao, L. Zheng, W.C. Liu, Z. Liu, M. Lei, L. Huang, X. Xie, X. Wang, Y. Chen, X. Yao, J. Peng, H. Gong, J.F. Griffith, Y. Huang, Y. Zheng, J.Q. Feng, Y. Liu, S. Chen, D. Xiao, D. Wang, J. Xiong, D. Pei, P. Zhang, X. Pan, X. Wang, K.M. Lee, and C.Y. Cheng, Phytomolecule icaritin incorporated PLGA/TCP scaffold for steroid-associated osteonecrosis: Proof-of-concept for prevention of 
hip joint collapse in bipedal emus and mechanistic study in quadrupedal rabbits. Biomaterials, 2015. 59: p. 125-143.

123. Zhang, J., S. Zhao, Y. Zhu, Y. Huang, M. Zhu, C. Tao, and C. Zhang, Threedimensional printing of strontium-containing mesoporous bioactive glass scaffolds for bone regeneration. Acta Biomater, 2014. 10(5): p. 2269-2281.

124. Probst, F.A., D.W. Hutmacher, D.F. Muller, H.G. Machens, and J.T. Schantz, [Calvarial reconstruction by customized bioactive implant]. Handchirurgie, Mikrochirurgie, plastische Chirurgie, 2010. 42(6): p. 369-373.

125. Esposito Corcione, C., F. Gervaso, F. Scalera, F. Montagna, T. Maiullaro, A. Sannino, and A. Maffezzoli, 3D printing of hydroxyapatite polymer-based composites for bone tissue engineering, in Journal of Polymer Engineering. 2017. p. 741-746. 


\title{
Chapter 3 - Preparation and mechanical properties of photo- crosslinked poly(trimethylene carbonate) and nano- hydroxyapatite composites
}

Mike A. Geven ${ }^{\mathrm{a}}$, Davide Barbieri ${ }^{\mathrm{b}}$, Huipin Yuan ${ }^{\mathrm{b}}$, Joost D. de Bruijn ${ }^{\mathrm{a}, \mathrm{b}}$ and Dirk W. Grijpma $^{\mathrm{a}}$

${ }^{a}$ Department of Biomaterials Science and Technology, University of Twente, Enschede, The Netherlands

${ }^{\mathrm{b}}$ Kuros BioSciences BV, Bilthoven, The Netherlands

\begin{abstract}
Composite materials of photo-crosslinked poly(trimethylene carbonate) and nanoscale hydroxyapatite were prepared and their mechanical characteristics for application as orbital floor implants were assessed. The composites were prepared by solvent casting poly(trimethylene carbonate) macromers with varying amounts of nano-hydroxyapatite and subsequent photo-crosslinking. The incorporation of the nano-hydroxyapatite into the composites was examined by thermogravimetric analysis, scanning electron microscopy and gel content measurements. The mechanical properties were investigated by tensile testing and trouser tearing experiments. Our results show that nano-hydroxyapatite particles can readily be incorporated into photo-crosslinked poly(trimethylene carbonate) networks. Compared to the networks without nano-hydroxyapatite, incorporation of $36.3 \mathrm{wt} \%$ of the apatite resulted in an increase of the E modulus, yield strength and tensile strength from 2.2 $\mathrm{MPa}$ to $51 \mathrm{MPa}, 0.5$ to $1.4 \mathrm{~N} / \mathrm{mm}^{2}$ and from 1.3 to $3.9 \mathrm{~N} / \mathrm{mm}^{2}$, respectively. We found that composites containing $12.4 \mathrm{wt} . \%$ nano-hydroxyapatite had the highest values of strain at break, toughness and average tear propagation strength $\left(376 \%, 777 \mathrm{~N} / \mathrm{mm}^{2}\right.$ and $3.1 \mathrm{~N} / \mathrm{mm}^{2}$, respectively).
\end{abstract}




\section{Introduction}

Blow-out fractures of the orbital floor are common injuries in traffic accidents or assaults, caused by excessive force applied to the infraorbital rim[1]. Restoring the original volume of the orbit and repositioning the globe are very important. In practice, this is done by covering the fracture site with an implant[1]. The current clinically used implants consist of autografts and allografts as well as of alloplastic materials such as hydroxyapatite ceramics, titanium, silicone rubber, ultra high density polyethylene, poly(lactic acid) and hydroxyapatite/polyethylene composites[2]. Ideally, an implant material is biocompatible, mechanically stable enough to support the orbital contents, osteoinductive and bioresorbable with a minimal foreign body response.

The limited availability and the possibility for disease transmittance associated with autografts and allografts decrease their appeal as implant materials[2]. Drawbacks of several of the alloplastic materials are sub-optimal mechanical properties, limited potential for bone ingrowth or their non-degradability. For these reasons, composites of synthetic, degradable and biocompatible polymers and calcium phosphates have been investigated for bone tissue engineering and orbital floor repair. Encouraging results have been achieved with composites of poly(L-lactide), poly(L-lactide-co-glycolide) and poly( $\varepsilon$-caprolactone) with several calcium phosphates such as hydroxyapatite, $\beta$-tricalcium phosphate $(\beta$-TCP) and biphasic calcium phosphate[2-12].

Although these polyester-based composites have shown good results, problems may arise from the significant amounts of acidic degradation products that can be formed. Furthermore, the average molar mass of polyesters will decrease during degradation resulting in decreasing mechanical properties[13]. Recently, composites of linear noncrosslinked poly(trimethylene carbonate) (PTMC) and $\beta$-TCP have been investigated as potential orbital floor implants. PTMC is a flexible, amorphous polymer that degrades enzymatically through surface erosion in presence of macrophages. This surface erosion mechanism produces non-acidic degradation products and the mechanical properties of PTMC are maintained over time as the average molar mass remains constant[14]. It has been shown that composites of PTMC with $\beta$-TCP had sufficient mechanical properties for the reconstruction of large orbital floor defects $\left(5.5 \mathrm{~cm}^{2}\right)[15]$. Furthermore, implantation of composites of PTMC and biphasic calcium phosphate in sheep orbital floor defects resulted in newly formed bone and excellent osseous integration with the surrounding bone[16]. Although these PTMC composites were shapeable, an exact anatomical fit to the orbital floor defect would be most desirable for the optimal orbital floor reconstruction and repositioning of the orbital contents[2].

Microstereolithography is an additive manufacturing process based on photopolymerization. It can reach precisions of $5 \mu \mathrm{m}$ and may allow for the fabrication of such precisely fitting implants[17]. For this we have prepared photo-curable three-armed PTMC macromers with methacrylate end-groups (PTMC-MA). PTMC-MA based resins with varying amounts of nano-hydroxyapatite were then prepared and photo-crosslinked to obtain PTMC network composites that may have osteoinductive properties[18]. 


\section{Materials and methods}

\subsection{Materials}

All materials were used as received unless stated otherwise. ForYou Medical Devices Ltd. provided the trimethylene carbonate (TMC) used for this work. The nano-hydroxyapatite powder consisted of 5 to $25 \mu \mathrm{m}$ sized agglomerates of needle-like hydroxyapatite crystals of 200 to $400 \mathrm{~nm}$ long and 20 to $50 \mathrm{~nm}$ wide, and was prepared by Kuros BioSciences BV as previously reported[19]. Figure 1 shows an SEM image of the nano-hydroxyapatite as received, and the size distribution as determined using ImageJ. Tin(II) 2-ethylhexanoate $\left(\mathrm{Sn}(\mathrm{Oct})_{2}\right)$, hydroquinone, triethylamine, methacrylic anhydride and deuterated chloroform were obtained from Sigma-Aldrich. 1,1,1-tris(hydroxymethyl)propane (trimethylolpropane) was from Fluka analytical and Lucirin ${ }^{\circledR}$ TPO-L was from BASF. Chloroform, propylene carbonate and calcium hydride were from Merck Millipore. Dichloromethane, from VWR chemicals, was dried over calcium hydride and subsequently distilled under dry $\mathrm{N}_{2}$.
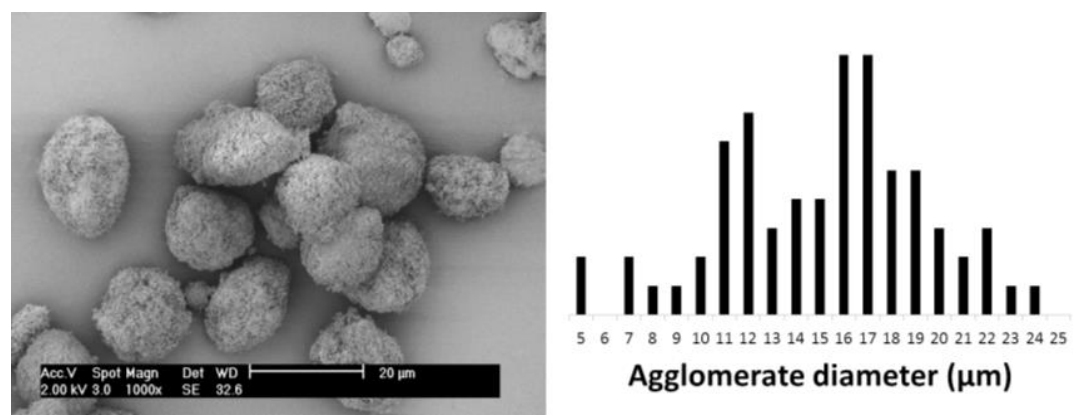

Figure 1. SEM image (left) and size distribution (right) of the nano-hydroxyapatite particles as were manufactured and received (scale bar: $20 \mu \mathrm{m}$ ).

\subsection{Methods}

2.2.1 Synthesis of PTMC-MA

3-armed PTMC was synthesized by ring-opening polymerization of TMC (figure 2). Under dry $\mathrm{N}_{2}$ atmosphere, a three-necked round-bottomed flask was charged with $49.8 \mathrm{~g}(0.49$ $\mathrm{mol}) \mathrm{TMC}$ and $0.67 \mathrm{~g}(5.0 \mathrm{mmol})$ trimethylolpropane as initiator. The polymerization was conducted at $140{ }^{\circ} \mathrm{C}$ for 19 hours, using $\mathrm{Sn}(\mathrm{Oct})_{2}$ as catalyst at a concentration of 0.13 wt.\%. After cooling to room temperature, the PTMC oligomer was dissolved in dried dichloromethane. Under dry $\mathrm{N}_{2}, 52.7 \mathrm{mg}$ of hydroquinone, $4.5 \mathrm{ml}(32 \mathrm{mmol})$ triethylamine and $4.6 \mathrm{ml}(31 \mathrm{mmol})$ methacrylic anhydride were added and the resulting solution was stirred in the dark for 5 days at room temperature. The solution was then extracted with demineralized water 3 times and the organic phase was precipitated in cold methanol. The precipitate was dried in the dark at ambient conditions overnight and for another 5 days at room temperature in vacuo.

The non-functionalized PTMC oligomer and the functionalized precipitated macromer were analyzed by ${ }^{1} \mathrm{H}-\mathrm{NMR}$ (Bruker Ascend 400/Avance III $400 \mathrm{MHz}$ NMR spectrometer) 
to determine the TMC conversion, the number average molecular mass $\left(M_{n}\right)$ and the degree of functionalization of PTMC-MA. Deuterated chloroform was used as the solvent.

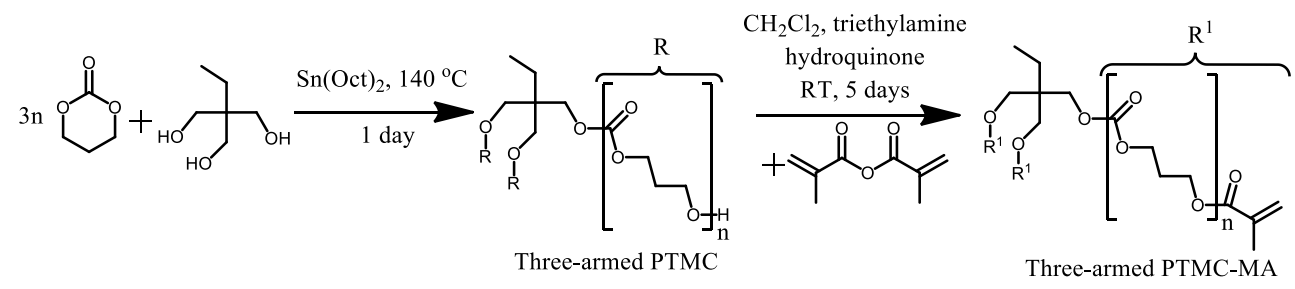

Figure 2. Synthesis of 3-armed PTMC and subsequent functionalization using methacrylic anhydride.

\subsubsection{Preparation of photo-crosslinked PTMC networks and composites}

Photo-crosslinked PTMC networks and composites were prepared with nanohydroxyapatite contents of $0,10,20$ and $35 \mathrm{wt} \%$. An amount of nano-hydroxyapatite powder was dispersed by vigorous stirring in chloroform. PTMC-MA (approximately 0.6 $\mathrm{g} / \mathrm{ml}$ chloroform) and hydroquinone (0.2 wt.\% relative to PTMC-MA) were then dissolved in the dispersion, forming a viscous solution. No sedimentation of nano-hydroxyapatite particles was observed after halting the stirring. Nonetheless, the dispersion was sonicated for 15 minutes to ensure homogeneity, and Lucirin ${ }^{\circledR}$ TPO-L photoinitiator (4 wt.\% relative to PTMC-MA) was mixed in. Immediately thereafter, the dispersion was cast on glass plates using a $1 \mathrm{~mm}$ casting knife. The formed films were dried to constant weight and subsequently photo-crosslinked under $\mathrm{N}_{2}$ in a UV-crosslinker (Ultra-Lum Electronic Ultraviolet Crosslinker) at $365 \mathrm{~nm}$ and $11 \mathrm{~mW} / \mathrm{cm}^{2}$ for 30 minutes. The photo-crosslinked films were extracted with a mixture of propylene carbonate/chloroform $(7 / 3 \mathrm{v} / \mathrm{v})$ twice and once with ethanol (no nano-hydroxyapatite particles were seen in the extraction media). The extracted composites were dried at ambient conditions overnight and consequently in vacuo until constant weight was achieved. The resulting films were 300 to $400 \mu \mathrm{m}$ thick.

\subsubsection{Characterization of photo-crosslinked PTMC and nano-hydroxyapatite composites}

The nano-hydroxyapatite content of the composites was determined by thermogravimetric analysis using a TGA 7 from Perkin Elmer. Samples were heated under $\mathrm{N}_{2}$-flow from 50 ${ }^{\circ} \mathrm{C}$ to $600{ }^{\circ} \mathrm{C}$ at a heating rate of $20{ }^{\circ} \mathrm{C} / \mathrm{min}$. All measurements were performed in threefold. The morphology of the PTMC networks and nano-hydroxyapatite composites was observed by scanning electron microscopy on an FEI/Philips XL-30 environmental Scanning Electron Microscope. The films were freeze-fractured and the cross-sections were imaged after gold-sputtering.

The gel content of the PTMC networks and the unreacted PTMC-MA sol fraction of the composites as synthesized were determined gravimetrically. The gel contents $G$ were determined in triplicate (correcting for the nano-hydroxyapatite content) according to equation 1 . 


$$
G=\frac{(1-x) m_{d}}{(1-x) m_{i}} * 100 \%
$$

The weight fraction of nano-hydroxyapatite in the PTMC network composite is $x$, the mass of the composite after extraction and drying is $m_{d}$, the mass of the cast films right before extraction is $m_{i}$.

\subsubsection{Mechanical characterization of photo-crosslinked networks and composites}

For tensile experiments, samples of $100 \times 5 \mathrm{~mm}^{2}$ were punched out of the extracted and dried films. A Zwick Z020 tensile tester with a $500 \mathrm{~N}$ load cell was used. The initial gripto-grip separation was $50 \mathrm{~mm}$ and the crosshead speed was $50 \mathrm{~mm} / \mathrm{min}$. All measurements were conducted in fivefold. From the stress-strain curves, the E modulus, the stress and elongation at break and the yield stress and elongation at yield were determined. The toughness of the materials was calculated from the area under the stress-strain curve.

Tear propagation experiments were conducted in analogy to ASTM 1938. Samples measuring $37.5 \times 12.5 \mathrm{~mm}^{2}$ with a $25 \mathrm{~mm}$ long slit were used. The tearing experiments were conducted at a crosshead speed of $250 \mathrm{~mm} / \mathrm{min}$. The average tear propagation strength and the maximum tear propagation strength values were determined in fivefold.

\section{Results and Discussion}

\subsection{Synthesis of PTMC-MA}

Three-armed PTMC was obtained by ring-opening polymerization of TMC using trimethylolpropane as initiator. The product was subsequently reacted with methacrylic anhydride to yield PTMC-MA. From the ${ }^{1} \mathrm{H}-\mathrm{NMR}$ spectrum of non-functionalized PTMC, figure 3, TMC monomer conversion was calculated by comparing the area of the TMC CO-O- $\mathbf{C H}_{2}-\mathrm{CH}_{2}-\mathbf{C H}_{2}-\mathrm{O}$ - peak at $4.46 \mathrm{ppm}$ with that of the PTMC -CO-O-C $\mathbf{C H}_{2}-\mathrm{CH}_{2}-\mathbf{C H}_{2}$ O- peak at $4.24 \mathrm{ppm}$. A value of approximately $98 \%$ was obtained. By comparing the area of the trimethylolpropane initiator $-\mathbf{C H}_{3}$ peak at $0.91 \mathrm{ppm}$ with the area of the PTMC methylene peak at $4.24 \mathrm{ppm}$, an $\mathrm{M}_{\mathrm{n}}$ value of $10.4 \mathrm{~kg} / \mathrm{mol}$ could be determined.

End-group functionalization of PTMC with methacrylic anhydride was confirmed by the appearance of the double bond proton peaks at $5.57 \mathrm{ppm}$ and $6.11 \mathrm{ppm}$ (figure 3). The degree of functionalization was determined from the area of these peaks and that of the trimethylolpropane-initiated PTMC $-\mathbf{C H}_{3}$ group. An average degree of functionalization of $80 \%$ could be calculated. 

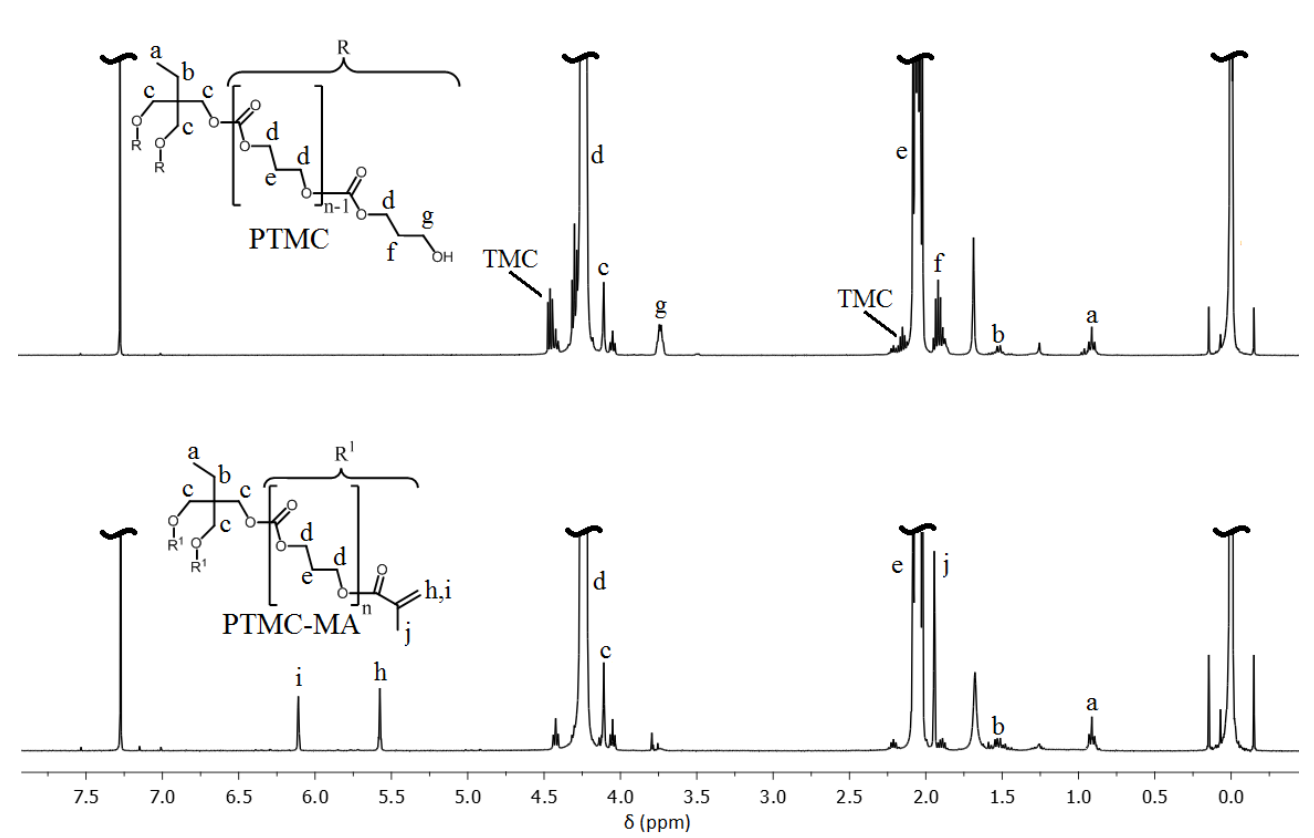

Figure 3. ${ }^{I} H$-NMR spectra of three-armed PTMC (top) and methacrylate end-group functionalized PTMC-MA (bottom).

\subsection{Composites of photo-crosslinked PTMC and nano-hydroxyapatite}

A series of photo-crosslinked PTMC and nano-hydroxyapatite composites were prepared by photo-crosslinking mixtures of PTMC macromers and nano-hydroxyapatite. The amount of nano-hydroxyapatite incorporated into the composites was determined by thermogravimetric analysis (TGA). The PTMC network part thermally decomposed at temperatures from 250 to $450{ }^{\circ} \mathrm{C}$ and the hydroxyapatite part remained unchanged at temperatures up to $600{ }^{\circ} \mathrm{C}$. TGA showed that composites with nano-hydroxyapatite contents of $12.4 \pm 0.1,20.8 \pm 0.1$ and $36.3 \pm 0.3$ wt.\% were prepared. This corresponds well with the amounts added to the macromer resin.

To assess the efficiency of the photo-crosslinking process, the gel content of the PTMC composites was determined. The gel content of photo-crosslinked PTMC was $77.0 \pm 0.8 \%$. The composites with 12.4, 20.8 and 36.3 wt.\% nano-hydroxyapatite had gel contents of $74.6 \pm 3.6,76.2 \pm 2.8$ and $84.6 \pm 0.7 \%$, respectively. This indicates that the presence of large amounts of nano-hydroxyapatite particles does not negatively affect the network formation by photo-crosslinking.

Cross-sections of the composite films were imaged using SEM (figure 4). The images showed that the cross-sections of the photo-crosslinked PTMC networks that did not contain nano-hydroxyapatite were quite smooth. Images of the composites with apatite showed that the nano-hydroxyapatite particles were well-dispersed. Across the crosssections of the films no gradient in particle loading could be detected in SEM images (data not shown). This indicates that sedimentation after film casting was minimal. The 
roughness of the cross-sections increases with increasing nano-hydroxyapatite content. The feature sizes of the rough surfaces are much smaller than the nano-hydroxyapatite particles used. It is likely that the mixing and sonication process in the PTMC macromer solutions leads to disintegration of these nano-hydroxyapatite agglomerates.

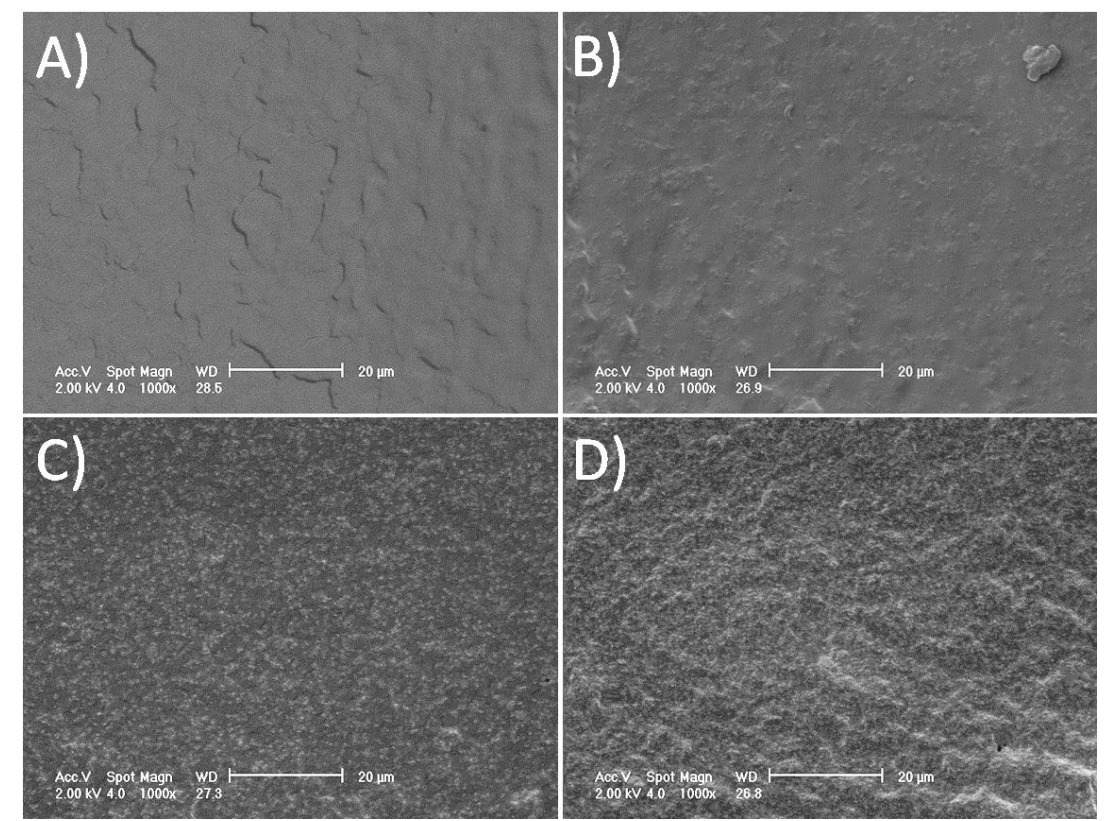

Figure 4. SEM images of cross-sections of photo-crosslinked PTMC (A) and composites containing 12.4 wt. $\%$ (B), $20.8 \mathrm{wt} \%$ (C), $36.3 \mathrm{wt} . \%$ (D) nano-hydroxyapatite (scale bar: $20 \mu \mathrm{m}$ ) (the impurity that can be discerned in figure $4 B$, is likely not a nano-hydroxyapatite agglomerate, given the morphology of the nano-hydroxyapatite particles shown in figure 1).

\subsection{Mechanical properties of photo-crosslinked PTMC and nano-hydroxyapatite composites}

Tensile experiments were conducted on composite network films to assess the effect of incorporating nano-hydroxyapatite into photo-crosslinked PTMC networks on their mechanical properties.

The data presented in table 1 shows that adding nano-hydroxyapatite to the PTMC network matrix has a significant effect on the mechanical properties of the composites. Compared to the PTMC network that does not contain nano-hydroxyapatite, incorporation of up to 36.3 wt.\% in general increases the E modulus, the yield strength and the tensile strength. The E modulus increased from 2.2 $\mathrm{MPa}$ to $51 \mathrm{MPa}$, the yield strength increased from 0.5 to 1.4 $\mathrm{N} / \mathrm{mm}^{2}$ and the strain at yield decreased from 30 to $4.4 \%$. The maximum tensile strength of the composites was increased upon nano-hydroxyapatite incorporation from 1.3 to 3.9 MPa. 
Table 1. Mechanical properties of photo-crosslinked PTMC networks and nano-hydroxyapatite composites as determined by tensile testing and trouser tearing experiments.

\begin{tabular}{|c|c|c|c|c|}
\hline & \multicolumn{4}{|c|}{ Nano-hydroxyapatite content (approximate) } \\
\hline & 0 wt.\% & 10 wt. $\%$ & 20 wt. $\%$ & 35 wt.\% \\
\hline E modulus (MPa) & $2.2 \pm 0.1$ & $4.9 \pm 0.2$ & $9.0 \pm 1.2$ & $51 \pm 7.4$ \\
\hline Yield strength $\left(\mathrm{N} / \mathrm{mm}^{2}\right)$ & $0.5 \pm 0.04$ & $0.7 \pm 0.03$ & $0.7 \pm 0.1$ & $1.4 \pm 0.2$ \\
\hline Strain at yield $(\%)$ & $30 \pm 1.7$ & $20 \pm 0.5$ & $11 \pm 0.4$ & $4.4 \pm 0.2$ \\
\hline Tensile strength $\left(\mathrm{N} / \mathrm{mm}^{2}\right)$ & $1.3 \pm 0.1$ & $3.2 \pm 0.4$ & $2.5 \pm 0.5$ & $3.9 \pm 0.3$ \\
\hline Strain at break $(\%)$ & $172 \pm 20$ & $376 \pm 38$ & $151 \pm 24$ & $100 \pm 8.9$ \\
\hline Toughness $\left(\mathrm{N} / \mathrm{mm}^{2}\right)$ & $141 \pm 14$ & $777 \pm 68$ & $204 \pm 34$ & $280 \pm 37$ \\
\hline $\operatorname{TPS}_{\mathrm{av}}(\mathrm{N} / \mathrm{mm})^{\mathrm{a}}$ & $1.2 \pm 0.04$ & $3.1 \pm 0.2$ & $1.6 \pm 0.2$ & $1.8 \pm 0.2$ \\
\hline $\mathrm{TPS}_{\max }(\mathrm{N} / \mathrm{mm})^{\mathrm{b}}$ & $1.4 \pm 0.04$ & $3.5 \pm 0.3$ & $1.8 \pm 0.2$ & $2.1 \pm 0.2$ \\
\hline
\end{tabular}

${ }^{\mathrm{a}}$ Average tear propagation strength

${ }^{\mathrm{b}}$ Maximum tear propagation strength

Interestingly, the table shows that PTMC networks with a nano-hydroxyapatite content of 12.4 wt.\% showed extraordinary behavior. Very high values of maximum tensile strength and strain at break of respectively $3.2 \mathrm{MPa}$ and $376 \%$ were found. Also the toughness (777 $\mathrm{N} / \mathrm{mm}^{2}$ ) of the specimens was exceptionally high.

This can be the result of the well-dispersed morphology of the small nano-hydroxyapatite agglomerates in the crosslinked PTMC matrix at this concentration, see figure 4B. In addition to their effect on the E modulus, the strain at break and toughness of crosslinked polymer/particle composites can increase significantly if the micro- and nano-sized particles are well-dispersed[20, 21].

Tear propagation experiments were conducted as well. The results in Table 1 show that the tear propagation strength (TPS) was much increased by incorporation of nanohydroxyapatite into the photo-crosslinked PTMC. Here too the highest values were found for the composite with a nano-hydroxyapatite content of $12.4 \mathrm{wt} . \%$. The average and maximum TPS increased from 1.2 to $3.1 \mathrm{~N} / \mathrm{mm}$ and from 1.4 to $3.5 \mathrm{~N} / \mathrm{mm}$, respectively. Currently we are investigating the behavior of photo-crosslinked PTMC and nanohydroxyapatite composites in biological environments.

\section{Conclusions}

We have prepared photo-crosslinked PTMC and composites thereof with nanohydroxyapatite, intended for orbital floor reconstruction. The nano-hydroxyapatite particles could readily be incorporated into a PTMC network matrix by mixing the nanoparticles with the photo-reactive PTMC macromer and subsequent photo-crosslinking. The nanohydroxyapatite particles did not have an adverse effect on the photo-crosslinking.

Tensile testing and tear propagation experiments demonstrated that the stiffness, strength and toughness of the composites are much increased by the incorporation of nano- 
hydroxyapatite. Also the resistance to tearing is considerably increased. Especially the composites with 12.4 wt.\% nano-hydroxyapatite had outstanding mechanical properties.

The attractive mechanical properties of photo-crosslinked PTMC and nano-hydroxyapatite composites make them interesting materials for use in the preparation of orbital floor implants. Precisely fitting implants can then be manufactured by microstereolithography.

\section{Acknowledgements}

This work was funded by the EU-China grant of the Seventh Framework Program of the European Union, Rapidos project number 604517.

\section{References}

1. Betz, M.W., J.F. Caccamese, D.P. Coletti, J.J. Sauk, and J.P. Fisher, Challenges associated with regeneration of orbital floor bone. Tissue Engineering Part B: Reviews, 2010. 16(5): p. 541-550.

2. Baino, F., Biomaterials and implants for orbital floor repair. Acta Biomaterialia, 2011. 7(9): p. 3248-3266.

3. McManus, A.J., R.H. Doremus, R.W. Siegel, and R. Bizios, Evaluation of cytocompatibility and bending modulus of nanoceramic/polymer composites. Journal of Biomedical Materials Research Part A, 2005. 72(1): p. 98-106.

4. Lickorish, D., L. Guan, and J.E. Davies, A three-phase, fully resorbable, polyester/calcium phosphate scaffold for bone tissue engineering: Evolution of scaffold design. Biomaterials, 2007. 28(8): p. 1495-502.

5. Ma, P.X., R.Y. Zhang, G.Z. Xiao, and R. Franceschi, Engineering new bone tissue in vitro on highly porous poly(alpha-hydroxyl acids)/hydroxyapatite composite scaffolds. Journal of Biomedical Materials Research, 2001. 54(2): p. 284-293.

6. Mathieu, L.M., T.L. Mueller, P.E. Bourban, D.P. Pioletti, R. Muller, and J.A. Manson, Architecture and properties of anisotropic polymer composite scaffolds for bone tissue engineering. Biomaterials, 2006. 27(6): p. 905-916.

7. Zhang, J.C., H.Y. Lu, G.Y. Lv, A.C. Mo, Y.G. Yan, and C. Huang, The repair of critical-size defects with porous hydroxyapatite/polyamide nanocomposite: an experimental study in rabbit mandibles. International Journal of Oral and Maxillofacial Surgery, 2010. 39(5): p. 469-477.

8. Davies, J.E., R. Matta, V.C. Mendes, and P.S. Perri de Carvalho, Development, characterization and clinical use of a biodegradable composite scaffold for bone engineering in oro-maxillo-facial surgery. Organogenesis, 2010. 6(3): p. 161-166.

9. Ciapetti, G., L. Ambrosio, L. Savarino, D. Granchi, E. Cenni, N. Baldini, S. Pagani, S. Guizzardi, F. Causa, and A. Giunti, Osteoblast growth and function in porous poly epsilon -caprolactone matrices for bone repair: a preliminary study. Biomaterials, 2003. 24(21): p. 3815-3824.

10. Hutmacher, D.W., J.T. Schantz, C.X. Lam, K.C. Tan, and T.C. Lim, State of the art and future directions of scaffold-based bone engineering from a biomaterials 
perspective. Journal of tissue engineering and regenerative medicine, 2007. 1(4): p. 245-260.

11. Kolk, A., J. Handschel, W. Drescher, D. Rothamel, F. Kloss, M. Blessmann, M. Heiland, K.D. Wolff, and R. Smeets, Current trends and future perspectives of bone substitute materials - from space holders to innovative biomaterials. Journal of Cranio-Maxillo-Facial Surgery, 2012. 40(8): p. 706-718.

12. Landes, C., A. Ballon, S. Ghanaati, A. Tran, and R. Sader, Treatment of Malar and Midfacial Fractures With Osteoconductive Forged Unsintered Hydroxyapatite and Poly-L-Lactide Composite Internal Fixation Devices. Journal of Oral and Maxillofacial Surgery, 2014. 72(7): p. 1328-1338.

13. $\mathrm{Wu}, \mathrm{L}$. and J. Ding, In vitro degradation of three-dimensional porous poly $(D, L-$ lactide-co-glycolide) scaffolds for tissue engineering. Biomaterials, 2004. 25(27): p. 5821-5830.

14. Zhang, Z., R. Kuijer, S.K. Bulstra, D.W. Grijpma, and J. Feijen, The in vivo and in vitro degradation behavior of poly(trimethylene carbonate). Biomaterials, 2006. 27(9): p. 1741-1748.

15. Van Leeuwen, A.C., R.R. Bos, and D.W. Grijpma, Composite materials based on poly(trimethylene carbonate) and beta-tricalcium phosphate for orbital floor and wall reconstruction. Journal of Biomedical Materials Research Part B: Applied Biomaterials, 2012. 100(6): p. 1610-1620.

16. van Leeuwen, A.C., H. Yuan, G. Passanisi, J.W. van der Meer, J.D. de Bruijn, T.G. van Kooten, D.W. Grijpma, and R.R. Bos, Poly(trimethylene carbonate) and biphasic calcium phosphate composites for orbital floor reconstruction: a feasibility study in sheep. European Cells \& Materials, 2014. 27: p. 81-96.

17. Wendel, B., D. Rietzel, F. Kuhnlein, R. Feulner, G. Hulder, and E. Schmachtenberg, Additive Processing of Polymers. Macromolecular Materials and Engineering, 2008. 293(10): p. 799-809.

18. Barbieri, D., A.J. Renard, J.D. de Bruijn, and H. Yuan, Heterotopic bone formation by nano-apatite containing poly(D,L-lactide) composites. European Cells \& Materials, 2010. 19: p. 252-261.

19. Barbieri, D., H.P. Yuan, X.M. Luo, S. Fare, D.W. Grijpma, and J.D. de Bruijn, Influence of polymer molecular weight in osteoinductive composites for bone tissue regeneration. Acta Biomaterialia, 2013. 9(12): p. 9401-9413.

20. Camargo, P.H.C., K.G. Satyanarayana, and F. Wypych, Nanocomposites: Synthesis, Structure, Properties and New Application Opportunities. Materials Research - Ibero-American Journal of Materials, 2009. 12(1): p. 1-39.

21. Zhang, J., B. Han, N.L. Zhou, J. Fang, J.A. Wu, Z.M. Ma, H. Mo, and J.A. Shen, Preparation and Characterization of Nano/Micro-Calcium Carbonate Particles/Polypropylene Composites. Journal of Applied Polymer Science, 2011. 119(6): p. 3560-3565. 


\title{
Chapter 4 - Fabrication of patient specific composite orbital floor implants by stereolithography
}

Mike A. Geven ${ }^{\mathrm{a}}$, Viktor Varjas ${ }^{\mathrm{b}}$, Lukas Kamer ${ }^{\mathrm{b}}$, Xinjiang Wang ${ }^{\mathrm{c}}$, Jian Peng ${ }^{\mathrm{c}}$, David Eglin ${ }^{\mathrm{b}}$ and Dirk W. Grijpma ${ }^{a}$

${ }^{a}$ Department of Biomaterials Science and Technology, University of Twente, Enschede, The Netherlands

${ }^{\mathrm{b}}$ AO Research Institute, Davos, Switzerland

${ }^{c}$ The Institute of Orthopaedics of Chinese People's Liberation Army General Hospital (301 Military Hospital), Beijing, People's Republic of China

\begin{abstract}
Fractures of the orbital floor are common in traffic accidents and assaults, and inadequate treatment can result in serious complications. Accurate anatomical reconstruction of the orbit using implants is the preferred treatment. Implants require degradability, adequate mechanical properties to support the orbital contents and osteoinductivity or -conductivity so that the implant is replaced by de novo bone over time.

Here we report on a semi-automatic process for the generation of virtual models of patient specific implants for orbital floor reconstruction. These models were generated using clinical computed tomography images of five clinical cases of orbital fracture. To fabricate accurately shaped implants based on the models, we utilized stereolithography, a high resolution additive manufacturing technique.

We prepared resins from bioresorbable, photo-curable functionalized poly(trimethylene carbonate) oligomers and osteoinductive nano-hydroxyapatite to manufacture composite implants. Incorporation of 40 wt.\% nano-hydroxyapatite into photo-crosslinked poly(trimethylene carbonate) lead to an increase of the E modulus, ultimate strength and toughness from 2.8 to $60 \mathrm{MPa}, 2.4$ to $7.1 \mathrm{~N} / \mathrm{mm}^{2}$ and 330 to $1671 \mathrm{~N} / \mathrm{mm}^{2}$ respectively. Additionally, water uptake increased from 0.8 to $7.3 \%$ and water contact angle decreased from 80 to $68^{\circ}$. Patient specific, homogeneous and mechanically stable implants can readily be prepared using these composite resins.
\end{abstract}




\section{Introduction}

Orbital fractures frequently occur in traffic accidents or assaults. Most often single wall fractures of the floor or two wall fractures of the floor combined with the medial wall occur. Such fractured orbital walls leave a void inferior or lateral to the ocular globe and are unlikely to provide sufficient support to the orbital contents[1]. Due to the limited thickness of the orbital floor, fracture results in thin bony fragments and contact between the bony edges of the fracture is limited. These factors provide a poor environment for selfhealing. Therefore, leaving such fractures untreated may result in serious complications such as diplopia and extra-ocular muscle dysfunction due to herniation of the orbital contents.

In order to reconstruct a fractured orbital floor, it is important to provide adequate support to the orbital contents and to accurately maintain the orbital volume[1, 2]. Especially in large fractures $\left(>1 \mathrm{~cm}^{2}\right)$ involving the floor and medial wall, reduced globe mobility and functional disorders may result from inadequate support and reconstruction. Autografts are considered the clinical gold standard for orbital reconstruction, although allografts and alloplastic materials are applied as well[3-7]. Since autografts and allografts are poorly shapeable, available in limited quantities and characterized by uncontrolled degradation, these are not ideal for orbital reconstruction. Use of degradable, osteoinductive or conductive and anatomically shaped alloplastic materials is preferred so that de novo bone may replace the implant and the orbital volume is restored.

Use of additive manufacturing (AM) for the fabrication of bone regenerating implants has gained much attention in literature over the past years. For an accurate anatomical reconstruction of the orbital floor and wall, use of AM for fabrication of implants based on computed tomography (CT) images of patient defects offers considerable advantages[8]. However, the virtual models of the orbits prepared using clinical CT images generally contain pseudo holes. These arise from the limited resolution of clinical CT images and are incompatible with AM. We have developed a computational workflow for the preparation of orbital floor implants from CT imaging data. We show that virtual models of patient specific implants (PSI) can accurately be produced using this workflow and AM.

For fabrication of bone regenerating implants, fused deposition modelling has often been applied as an AM technique. Composite materials with calcium phosphate are frequently utilized due to the bone forming properties of the ceramic component. Positive results have been achieved with composites of poly(L-lactide), poly(D,L-lactide), poly(L-lactide-coglycolide) or poly( $\varepsilon$-caprolactone) with hydroxyapatite or $\beta$-tricalcium phosphate[9-13]. Poly(trimethylene carbonate) (PTMC) is a polymer that degrades enzymatically through surface erosion, without acidic degradation products. Lack of acidic degradation products may be beneficial for bone formation and the surface erosion mechanism results in mechanical properties which are maintained over time[14]. Additionally, composites of PTMC with $\beta$-tricalcium phosphate showed promising results for orbital floor reconstruction in the past $[15,16]$. 
Stereolithography is a high resolution AM technique based on layer-by-layer photopolymerization or -crosslinking. It has been exploited for fabrication of tissue engineering scaffolds[17-22]. We prepared a resin for stereolithography based on photo-crosslinkable functionalized PTMC oligomers (macromers) and nano-hydroxyapatite (nHA). The nHA particles with nanometer scale features can be readily dispersed in polymer matrices, forming osteoinductive composite materials[23, 24].

By combining our workflow for generation of virtual models of PSI and fabrication of implants by stereolithography using the photo-crosslinkable PTMC/nHA composite resin, we aim to prepare orbital floor implants that optimally restore the orbital volume, adequately support the orbital contents and induce bone growth.

\section{Experimental}

\subsection{Materials}

Trimethylene carbonate (TMC) was obtained from Hiuzhou Foryou Medical Devices Co. Ltd.. nHa consisted of aggregates $(\varnothing=15 \mu \mathrm{m})$ of needle-like hydroxyapatite crystals of 200 to $400 \mathrm{~nm}$ long and 20 to $50 \mathrm{~nm}$ wide. It was provided by Kuros BioSciences BV and was prepared as previously reported[24]. 1,1,1-tris(hydroxymethyl)propane (trimethylolpropane) was from Fluka analytical. Tin(II) ethylhexanoate $\left(\mathrm{Sn}(\mathrm{Oct})_{2}\right)_{\text {, }}$ hydroquinone, triethylamine, methacrylic anhydride and deuterated chloroform were from Sigma-Aldrich. Lucirin ${ }^{\circledR}$ TPO-L photoinitiator was from BASF. Orasol Orange G dye was obtained from Ciba Specialty Chemicals. Propylene carbonate and calcium hydride were from Merck Millipore. Water used for this work was demineralized water purified by a MilliQ Advantage A10 system equipped with an $0.22 \mu \mathrm{m}$ Millipak ${ }^{\circledR}-40$ Express filter. Ethanol (99.8\%) was acquired from Atlas \& Assink Chemie BV. Dichloromethane and methanol were from VWR Chemicals. Dichloromethane was dried over calcium hydride and subsequently distilled under a dry $\mathrm{N}_{2}$ atmosphere. A non-degradable acrylate resin (PIC100) for stereolithography was acquired from Envisiontec. Materials were used as received unless stated otherwise.

\subsection{Generation of PSI models}

PSI computer models compatible with AM were generated using a semi-automatic workflow which merely required the user to define some landmarks describing the fracture shape and location. Five retrospective, routine preoperative CT scans of patients (2 females, 3 males, age between 10-54 years, AVG: 32, STD: 15) affected by unilateral orbital defects (3 orbital floor, 2 orbital floor/medial wall fractures) were obtained after approval from the board of ethics of the General Hospital of the People's Liberation Army in Beijing, P.R. China (06/05/2014). An orbital mean model was derived from 3D statistical modeling. The data was processed using Amira (FEI Visualization Sciences Group, Bordeaux, France) extended by custom modules using TCL scripting and C++ programing. Generated PSI computer models were eventually exported as .STL-files. 


\subsection{Synthesis of methacrylate end-group functionalized PTMC and formulation of resins}

Three-armed PTMC was synthesized by ring-opening polymerization of TMC initiated by trimethylolpropane. In a molar ratio of 96.4/1 (TMC/trimethylolpropane) these were charged in a flask under dry $\mathrm{N}_{2}$ atmosphere. Using 0.13 wt.\% $\mathrm{Sn}(\mathrm{Oct})_{2}$ as a catalyst, the polymerization was conducted at $130{ }^{\circ} \mathrm{C}$ for 2 days. The formed PTMC was thereafter dissolved in dichloromethane under a dry $\mathrm{N}_{2}$ atmosphere. A PTMC macromer (PTMCMA) with methacrylate end-groups was subsequently prepared by reaction with the hydroxyl end-groups. Under vigorous stirring $0.11 \mathrm{wt} . \%$ hydroquinone, triethylamine (9 $\mathrm{mol} / \mathrm{mol} \mathrm{PTMC}$ ) and methacrylic anhydride (9 mol/mol PTMC) were added to the PTMC solution. The functionalization reaction was conducted over 5 days after which the solution was precipitated in cold methanol. The precipitate was extracted in fresh, cold methanol to further purify the product. This was thereafter dried at room temperature in vacuo over 7 days.

The synthesized PTMC oligomer was analyzed by ${ }^{1} \mathrm{H}-\mathrm{NMR}$ (Bruker Ascend 400/Avance III $400 \mathrm{MHz}$ NMR spectrometer) to determine the number average molecular weight $\left(\mathrm{M}_{\mathrm{n}}\right)$ and the conversion of TMC. The PTMC-MA was also analyzed by ${ }^{1} \mathrm{H}-\mathrm{NMR}$ to determine the degree of functionalization (DF). All ${ }^{1} \mathrm{H}-\mathrm{NMR}$ analysis was performed in deuterated chloroform.

PTMC-MA and PTMC-MA/nHA resins with 5.2 wt.\% nHA (PTMC-MA-10) and 16.7 wt.\% nHA (PTMC-MA-40) were prepared in order to fabricate structures with a nHA content of 0,10 and 40 wt.\%, respectively. Table 1 gives an overview of the resin compositions. Resins were formed by dispersing calculated amounts of nHA in chloroform after which PTMC-MA $(0.1 \mathrm{~g} / \mathrm{ml})$ was dissolved in the dispersion. The resulting solution was mechanically stirred and precipitated in cold methanol to form a homogeneous composite. This was dried in vacuo at room temperature to constant weight. A non-reactive diluent for stereolithography, propylene carbonate, was then added and the resulting viscous mixture was heated to $60{ }^{\circ} \mathrm{C}$ and mixed in the dark over 3 days. The mixture was subsequently sonicated for $30 \mathrm{~min}$. at $60{ }^{\circ} \mathrm{C}$ and Lucirin ${ }^{\circledR}$ TPO-L and Orasol Orange G was mixed in.

The viscosity of the PTMC-MA and PTMC-MA/nHA resins was determined using a Brookfield DV-E rotating spindle viscometer equipped with a small sample adapter. Viscosities were determined in triplicate at $0.6 \mathrm{rpm}$ and room temperature. 
Fabrication of patient specific composite orbital floor implants by stereolithography

Table 1. Composition of the PTMC-MA resin and the PTMC-MA/nHA resins.

\begin{tabular}{lccc}
\hline & \multicolumn{3}{c}{ Resin } \\
\cline { 2 - 4 } Component & PTMC-MA & PTMC-MA/nHA-10 & PTMC-MA/nHA-40 \\
\hline PTMC-MA (wt.\%) & 0 & 47.4 & 25.1 \\
nHA (wt.\%) & 40 & 5.2 & 16.7 \\
Propylene carbonate (wt.\%) & 5 & 47.4 & 58.2 \\
Lucirin ${ }^{\circledR}$ TPO-L (wt.\%*) & 0.15 & 5 & 5 \\
Orasol Orange G (wt.\%*) & & 0.12 & 0.08 \\
\hline
\end{tabular}

*: relative to PTMC-MA

\subsection{Stereolithography fabrication of implants}

All .STL-files of the PSI computer models were modified with a supporting structure to ensure complete attachment to the build platform in the stereolithography process. A commercially available NURBS surface modelling software (Rhinoceros 4, Robert McNeel $\&$ Associates) was used for these modifications. The supporting structures consisted of a rectangular base of $1 \mathrm{~mm}$ thick connected to the PSI computer model via evenly distributed pillars.

Implants were prepared using an Envisiontec Perfactory ${ }^{3}{ }^{\circledR} \mathrm{SXGA}^{+}$Standard UV system. Irradiation times of 9 seconds per layer were utilized at $180 \mathrm{~mW} / \mathrm{dm}^{2}$. Using the PIC100 resin, implants were fabricated using the 5 virtual models of the PSI. These structures were extracted and washed with acetone and supporting parts were thereafter removed.

Using PTMC-MA and PTMC-MA/nHA composite resins, a PSI model as well as thin films (300-450 $\mu \mathrm{m}$ thick) were produced by stereolithography. To remove the non-reactive diluent, the prepared implants and thin films were extracted in propylene carbonate/ethanol $(1 / 1 \mathrm{v} / \mathrm{v})$ which was refreshed twice in 2 days. Thereafter the extraction medium was refreshed daily, decreasing the propylene carbonate content by $10 \mathrm{vol} \%$ in each refreshing. After extraction in ethanol, the structures and thin films were dried under ambient conditions until constant weight.

\subsection{Characterization of implants and photo-crosslinked thin films prepared by stereolithography}

The homogeneity of PTMC-MA and PTMC-MA/nHA structures fabricated by stereolithography was investigated by high resolution SEM. Freeze-fractured cross-sections were imaged using a Zeiss Merlin scanning electron microscope with high efficiency secondary electron detector at 0.85 to $1.4 \mathrm{kV}$.

The mechanical properties of photo-crosslinked PTMC-MA and PTMC-MA/nHA composites were assessed by tensile experiments. Samples measuring $75 \times 5 \mathrm{~mm}^{2}(\mathrm{n}=5)$ were punched out of the thin films and tested using a Zwick Z020 tensile tester equipped with a $500 \mathrm{~N}$ load cell. The initial grip-to-grip separation was $50 \mathrm{~mm}$ and the crosshead speed was $50 \mathrm{~mm} / \mathrm{min}$. From the engineering stress-strain curves, the E modulus, ultimate tensile strength, elongation at break, yield strength and elongation at yield were 
determined. The toughness of the materials was calculated from the area under the stressstrain curves.

For water uptake (WU) experiments, disk shaped samples $(n=3)$ with an $8 \mathrm{~mm}$ diameter were punched out of the thin films. These were immersed in water for 48 hours at room temperature. WU was determined by measuring the dry weight of the samples $\left(\mathrm{m}_{\mathrm{dry}}\right)$ before immersion and by weighing samples after immersion and dabbing $\left(\mathrm{m}_{\text {wet }}\right)$. WU was calculated using equation 1 .

$$
W U=\frac{\left(m_{w e t}-m_{d r y}\right)}{m_{d r y}} * 100 \%
$$

Contact angle measurements were carried out using a Dataphysics contact angle measurement system OCA 20 equipped with an electronic syringe module. Drops of $2 \mu 1$ were deposited on thin films $(n=3)$ at room temperature.

\section{Results \& discussion}

\subsection{Implant generation}

An overview of the entire generation procedure of PSI models is given in figure 1. The procedure began with the pre-processing of the CT images by applying noise-reduction, thresholding of the bone and the filtering of small floating components which might remain after the thresholding from which a 3D orbital model was generated (figure 1A). This preliminary 3D model is affected by pseudo holes, which occur due to the limited resolution of the $\mathrm{CT}$ imaging system (partial volume averaging). These pseudo holes were successfully eliminated using a complex automatic reconstruction procedure by adapting the orbital mean model using a series of rigid, affine and non-linear registrations (figure 1B). Then the reconstructed intact side was automatically mirrored and registered to the fractured side allowing rigid and isotropic scaling motion in order to model the potential asymmetry of the skull, thus volume difference, of the orbits. Landmarks were manually placed on the 3D model of the affected side describing the properties of the fracture (figure 1C). Relying on the mirrored and reconstructed intact side and on the landmarks provided, a PSI was automatically generated covering the defect and shaped as the corresponding intact region (figure 1D). In all five cases, virtual models of PSIs were generated and shaped based on manually defined fracture descriptor landmarks. The required thickness of the implant can automatically be calculated as a function of the defect size and the material properties of the implant, which can be set manually by the user. 

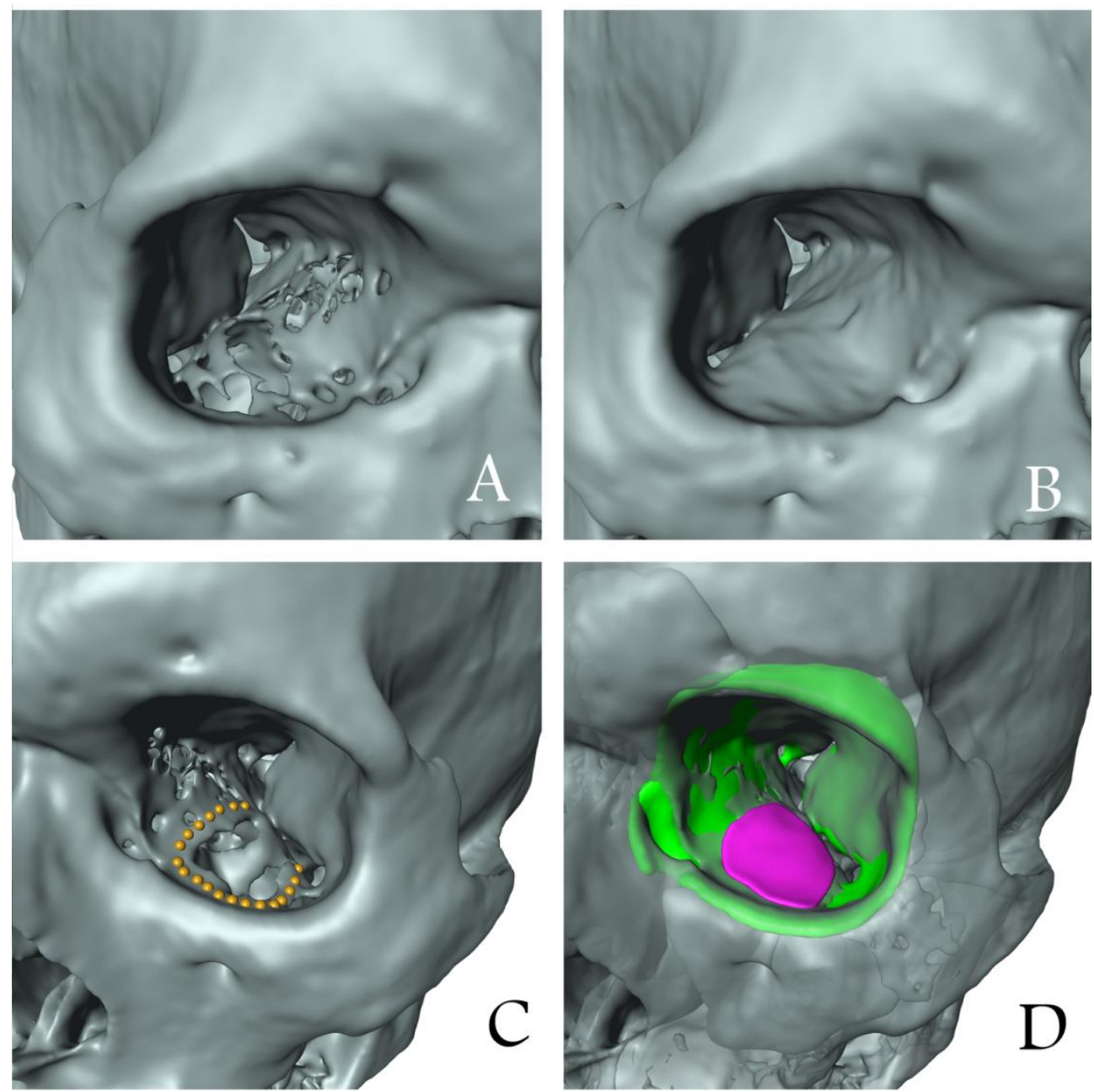

Figure 1. The preliminary $3 D$ model of the orbit $(A)$ and the result of the automatic pseudo-hole elimination procedure $(B)$. Landmarks describing the fracture pattern and location $(C)$ and the magenta colored result of the automatic implant generation $(D)$.

\subsection{Orbital floor PSI fabricated by stereolithography}

Using the PIC100 resin, PSI could be accurately fabricated. During layer-by-layer fabrication by stereolithography a voxel depth of $50 \mu \mathrm{m}$ was achieved. PSI models were therefore fabricated with a layer thickness of $50 \mu \mathrm{m}$. Figure 2 depicts the PSI models and the respective implant structures prepared with the PIC100 resin after removal of the supports. It can be seen that in all cases, the implant shape corresponds well with that of the model. 


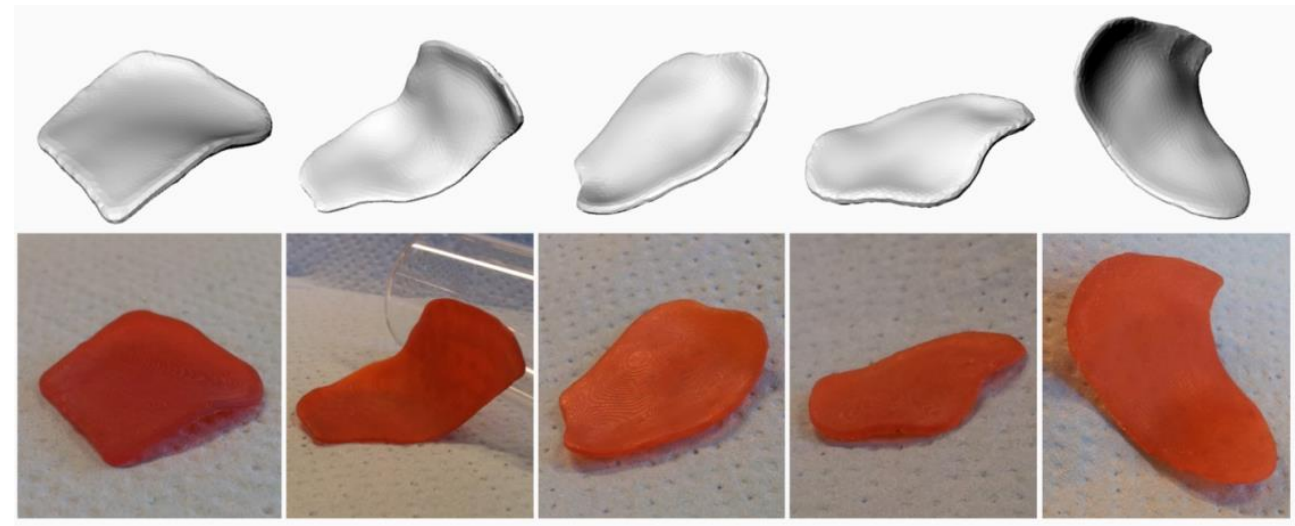

Figure 2. PSI models (top row) and corresponding implant structures (bottom row) fabricated by stereolithography using the PIC100 resin.

\subsection{Characterization of PTMC-MA and composite stereolithography resins}

Three-armed PTMC was prepared by ring-opening polymerization of TMC initiated by trimethylolpropane. The conversion of TMC and the $\mathrm{M}_{\mathrm{n}}$ of PTMC was determined by ${ }^{1} \mathrm{H}$ NMR as previously reported[25]. The TMC conversion was $99 \%$ and 3-armed PTMC with an $\mathrm{M}_{\mathrm{n}}$ of $9800 \mathrm{~g} / \mathrm{mol}$ was formed. The PTMC end-groups were functionalized with methacrylic anhydride to form PTMC-MA and the DF was determined from ${ }^{1} \mathrm{H}-\mathrm{NMR}$ spectra as previously reported as well[25]. A DF higher than $99 \%$ was determined.

The viscosity of PTMC-MA and PTMC-MA/nHA composite resins were determined to assess their processability. The viscosity was $60.8 \pm 0.9,57.5 \pm 1.0$ and $71.9 \pm 2.2 \mathrm{~Pa} \cdot \mathrm{s}$ for the PTMC-MA, PTMC-MA/nHA-10 and PTMC-MA/nHA-40 resin respectively. Inclusion of much nHA in the resins increased the viscosity, even though increasing amounts of diluent are added to control it. At the highest nHA content, implants could nonetheless be readily prepared.

\subsection{PTMC-MA and PTMC-MA/nHA composite implants prepared by stereolithography}

Using the PTMC-MA and PTMC-MA/nHA composite resins one of the PSI models was fabricated. Fabrication with a layer thickness of $50 \mu \mathrm{m}$ was again achieved.

Figure 3 shows the virtual PSI model and the implants fabricated with the PTMC-MA and PTMC-MA/nHA composite resins after support removal. The implant shape matched that of the virtual PSI model well. Only when PTMC-MA/nHA-40 resin was used, small defects could be discerned on the top surface of the implants. This is likely caused by dislodging of larger nHA aggregates. 

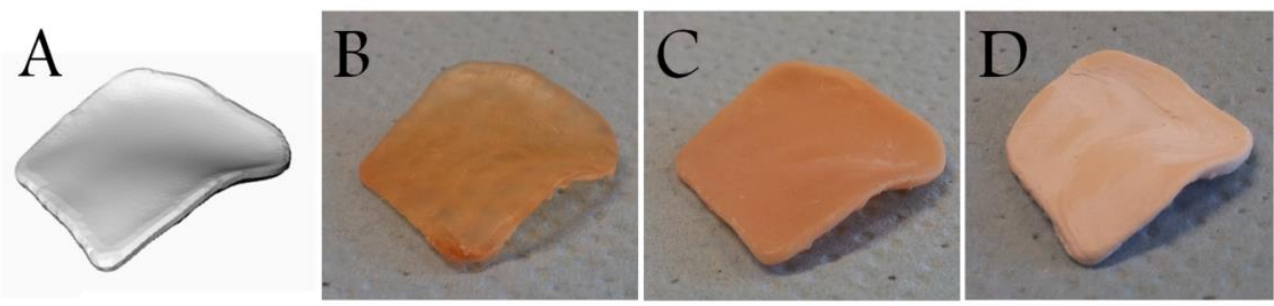

Figure 3. PSI model (A) and respective implant structures of PTMC-MA (B), PTMC-MA/nHa composite with 10 wt.\% $n H A(C)$ and 40 wt.\% $n H A(D)$.

\subsection{Characterization of photo-crosslinked PTMC-MA and PTMC-MA/nHA composites}

Using the PTMC-MA, PTMC-MA/nHA-10 and PTMC-MA/nHA-40 resins, thin films containing 0,10 and $40 \mathrm{wt} . \% \mathrm{nHA}$ were prepared by stereolithography. Homogeneity of photo-crosslinked PTMC-MA and PTMC-MA/nHA composites was investigated by imaging freeze-broken cross-sections of implant structures by high resolution SEM as shown in figure 4.

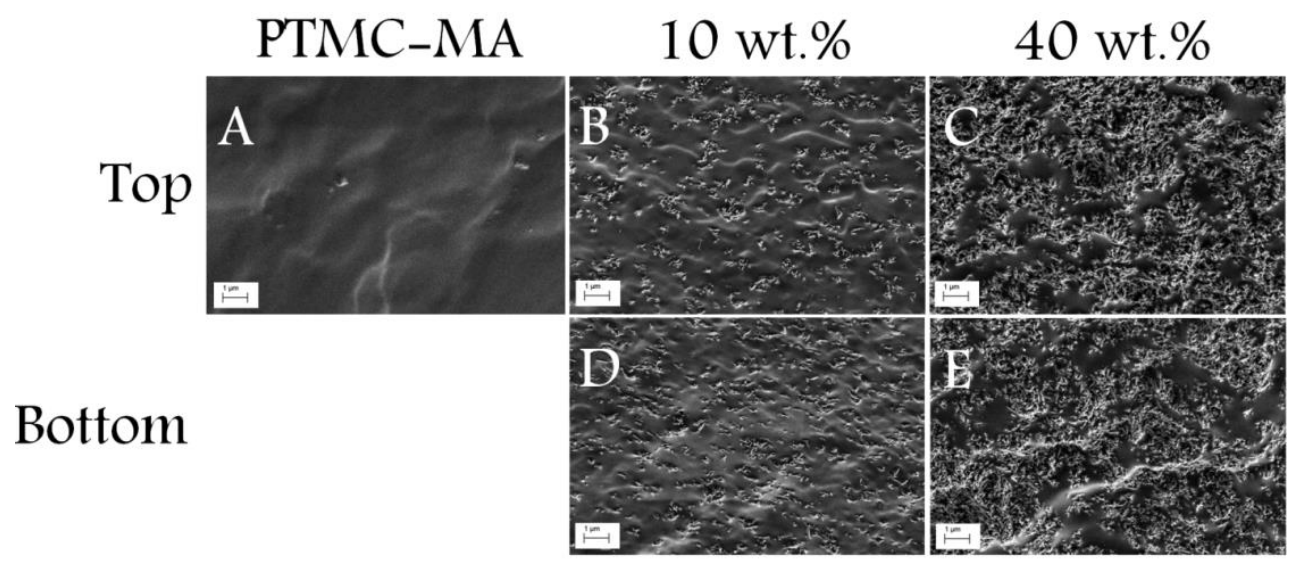

Figure 4. SEM images of cross-sections of implant materials of photo-crosslinked PTMC-MA (A), PTMCMA/nHA with 10 wt.\% nHA (B and D) and PTMC-MA/nHA with 40 wt.\% nHA (C and E). Scale bar is $1 \mu \mathrm{m}$.

The cross-section of photo-crosslinked PTMC-MA is smooth with only a few defects caused by the freeze-fracturing process. The cross-sections of PTMC-MA/nHA with 10 wt.\% nHA clearly showed the presence of the inorganic particles. The needle-like hydroxyapatite crystals were present in agglomerates of generally less than $1 \mu \mathrm{m}$ in size. These aggregates were homogeneously distributed and even separate needle-like crystals could be discerned in the polymer network matrix. A few larger aggregates measuring maximally $2 \mu \mathrm{m}$ in diameter at most could also be discerned. The cross-sections of PTMC$\mathrm{MA} / \mathrm{nHA}$ with $40 \mathrm{wt} \%$ nHA content were characterized by large patches of adjacent nHA particles, marbled with crosslinked PTMC-MA patches. This is distinctive for the 
increasing connectivity of the inorganic phase with an increasing nHA content. In a few areas rounded aggregates with a diameter between 5 and $30 \mu \mathrm{m}$ were also present in this composite. In both PTMC-MA/nHA composites, no significant differences were detected between the homogeneity at the top and bottom of the sample (see figure 4), indicating that the resins form a stable nHA dispersion without sedimentation over a prolonged period of time. Even when leaving the resin for more than a week before fabricating the PSI model, the cross-sections of the structures were equally homogeneous as the ones in figure 4 (data not shown).

The mechanical properties of photo-crosslinked PTMC-MA and PTMC-MA/nHA composite materials were investigated by tensile experiments; the results are given in table 2. By varying the nHA content from 0 to $40 \mathrm{wt} . \%$ the $\mathrm{E}$ modulus, yield strength and ultimate tensile strength of the implant materials increases. The E-modulus increases from 2.8 to $60 \mathrm{MPa}$, the yield strength from 0.6 to $1.7 \mathrm{~N} / \mathrm{mm}^{2}$ and the ultimate tensile strength from 2.4 to $7.1 \mathrm{~N} / \mathrm{mm}^{2}$. From the data in table 2, it is clear that the photo-crosslinked PTMC-MA and PTMC-MA/nHA composites formed by stereolithography become tougher with increasing nHA content. This is not necessarily an effect of the nHA content only, but can also be caused by the increased amount of diluent present during the photocrosslinking[26-28]. The strain at break increases from 239 to $410 \%$ which is accompanied by a large increase in toughness from 330 to $1671 \mathrm{~N} / \mathrm{mm}^{2}$ when the diluent content of the resin is increased from 40 to $58.2 \mathrm{wt} . \%$.

Table 2. Properties of photo-crosslinked PTMC-MA and PTMC-MA/nHA composite materials

\begin{tabular}{lccc}
\hline & \multicolumn{3}{c}{ nHA content } \\
\cline { 2 - 4 } Property & 0 wt.\% & $10 \mathrm{wt} \%$ & $40 \mathrm{wt} . \%$ \\
\hline E modulus $(\mathrm{MPa})$ & $2.8 \pm 0.1$ & $4.8 \pm 0.3$ & $60 \pm 6.6$ \\
Yield strength $\left(\mathrm{N} / \mathrm{mm}^{2}\right)$ & $0.6 \pm 0.03$ & $0.7 \pm 0.04$ & $1.7 \pm 0.1$ \\
Strain at yield $(\%)$ & $26 \pm 0.3$ & $19 \pm 0.9$ & $3.8 \pm 0.3$ \\
Ultimate tensile strength $\left(\mathrm{N} / \mathrm{mm}^{2}\right)$ & $2.4 \pm 0.3$ & $4.8 \pm 1.0$ & $7.1 \pm 0.6$ \\
Strain at break $(\%)$ & $239 \pm 33$ & $315 \pm 33$ & $410 \pm 35$ \\
Toughness $\left(\mathrm{N} / \mathrm{mm}^{2}\right)$ & $330 \pm 71$ & $722 \pm 156$ & $1671 \pm 199$ \\
Water uptake $(\%)$ & $0.8 \pm 0.0$ & $0.7 \pm 0.4$ & $7.3 \pm 0.7$ \\
Water contact angle $\left({ }^{\circ}\right)$ & $80 \pm 3.9$ & $87 \pm 5.5$ & $68 \pm 4.4$ \\
\hline
\end{tabular}

To assess the hydrophilicity of photo-crosslinked PTMC-MA and PTMC-MA/nHA composites, the water uptake of the implant materials and water contact angle on their surface was determined. The water uptake and contact angle data is also presented in table 2. Both photo-crosslinked PTMC-MA and PTMC-MA/nHA with $10 \mathrm{wt} \%$ nHA content are quite hydrophobic and therefore have a low water uptake $(0.8$ and $0.7 \%$ respectively) and relatively high water contact angles ( 80 and $87^{\circ}$ respectively). It seems that a low nHA content does not significantly affect the interaction of the implant material with water. 
When higher amounts of nHA are incorporated however, an increase of the water uptake is seen to $7.3 \%$ for photo-crosslinked PTMC-MA/nHA with 40 wt.\% nHA. Also a slight decrease of the water contact angle to $68^{\circ}$ is seen. At such high nHA contents, the hydrophilic inorganic particles may be exposed at the implant material surface as well as being linked within the polymer matrix (see also figure 4 ). This may facilitate enhanced interaction with water and swelling and therefore improved interaction with biological fluids. Adjoined particles are not present in photo-crosslinked composites of PTMC$\mathrm{MA} / \mathrm{nHA}$ with $10 \mathrm{wt} \% \mathrm{nHA}$ content and therefore there is no enhanced interaction with water.

\section{Conclusions}

Virtual models of PSI for the reconstruction of orbital fractures were generated using a semi-automated process, and implants were fabricated accordingly using stereolithography. The shapes of the fabricated implants corresponded well to the shape of models. Additionally, resins of PTMC-MA and PTMC-MA/nHA composite resins were prepared. Using these resins, implants were fabricated from a PSI model. The shape of these fabricated implants also matches the PSI model very well. Physical properties of photocrosslinked PTMC-MA and PTMC-MA/nHA composites were determined. It was shown that the stereolithography process typically allowed for fabrication of homogeneous photocrosslinked composites. The stiffness, ultimate tensile strength and toughness of photocrosslinked PTMC-MA and PTMC-MA/nHA composites increased significantly with increasing nHA content. The interaction of these materials with water was increased at a high nHA contents.

\section{Acknowledgements}

The authors acknowledge the funding by the Chinese NSFC-DG-RTD Joint Scheme (Project No. 51361130034) and the EU FP7 RAPIDOS project (Grant No. NMP3-SL2013-604517).

\section{References}

1. Betz, M.W., J.F. Caccamese, D.P. Coletti, J.J. Sauk, and J.P. Fisher, Challenges associated with regeneration of orbital floor bone. Tissue Engineering Part B: Reviews, 2010. 16(5): p. 541-550.

2. Jaquiery, C., C. Aeppli, P. Cornelius, A. Palmowsky, C. Kunz, and B. Hammer, Reconstruction of orbital wall defects: critical review of 72 patients. International Journal of Oral \& Maxillofacial Surgery, 2007. 36(3): p. 193-199.

3. Baino, F., Biomaterials and implants for orbital floor repair. Acta Biomaterialia, 2011. 7(9): p. 3248-3266.

4. Landes, C., A. Ballon, S. Ghanaati, A. Tran, and R. Sader, Treatment of malar and midfacial fractures with osteoconductive forged unsintered hydroxyapatite and 
poly-L-lactide composite internal fixation devices. Journal of oral and maxillofacial surgery, 2014. 72(7): p. 1328-1338.

5. Potter, J.K. and E. Ellis, Biomaterials for reconstruction of the internal orbit. Journal of oral and maxillofacial surgery, 2004. 62(10): p. 1280-1297.

6. Ellis, E., 3rd and E. Messo, Use of nonresorbable alloplastic implants for internal orbital reconstruction. Journal of oral and maxillofacial surgery, 2004. 62(7): p. 873-881.

7. Prowse, S.J., P.M. Hold, R.F. Gilmour, U. Pratap, E. Mah, and F.W. Kimble, Orbital floor reconstruction: a case for silicone. A 12 year experience. Journal of plastic, reconstructive \& aesthetic surgery, 2010. 63(7): p. 1105-1109.

8. Metzger, M.C., R. Schon, C. Zizelmann, N. Weyer, R. Gutwald, and R. Schmelzeisen, Semiautomatic procedure for individual preforming of titanium meshes for orbital fractures. Plastic and reconstructive surgery, 2007. 119(3): p. 969-976.

9. Sa, M.W., S.E. Kim, Y.P. Yun, H.R. Song, and J.Y. Kim, Fabrication of Hybrid Scaffolds by Polymer Deposition System and Its In-vivo Evaluation with a Rat Tibial Defect Model. Tissue Engineering and Regenerative Medicine, 2014. 11(6): p. 439-445.

10. Probst, F.A., D.W. Hutmacher, D.F. Muller, H.G. Machens, and J.T. Schantz, [Calvarial reconstruction by customized bioactive implant]. Handchirurgie, Mikrochirurgie, plastische Chirurgie, 2010. 42(6): p. 369-273.

11. Shor, L., J. Gordon, Y. An, S. Güçeri, and W. Sun, Precision Extruding Deposition of Polycaprolactone and Composite Polycaprolactone/Hydroxyapatite Scaffolds for Tissue Engineering. Proceedings of the IEEE 32nd Annual Northeast Bioengineering Conference, 2006. 1: p. 73-74.

12. Pang, L., W. Hao, M. Jiang, J. Huang, Y. Yan, and Y. Hu, Bony defect repair in rabbit using hybrid rapid prototyping polylactic-co-glycolic acid/betatricalciumphosphate collagen I/apatite scaffold and bone marrow mesenchymal stem cells. Indian journal of orthopaedics, 2013. 47(4): p. 388-394.

13. Roh, H.S., S.W. Myung, S.-C. Jung, and B.-H. Kim, Fabrication of 3D Scaffolds with Nano-Hydroxyapatite for Improving the Preosteoblast Cell-Biological Performance. Journal of Nanoscience and Nanotechnology, 2015. 15(8): p. 55855588.

14. Zhang, Z., R. Kuijer, S.K. Bulstra, D.W. Grijpma, and J. Feijen, The in vivo and in vitro degradation behavior of poly(trimethylene carbonate). Biomaterials, 2006. 27(9): p. 1741-1748.

15. Van Leeuwen, A.C., R.R. Bos, and D.W. Grijpma, Composite materials based on poly(trimethylene carbonate) and beta-tricalcium phosphate for orbital floor and wall reconstruction. Journal of Biomedical Materials Research Part B: Applied Biomaterials, 2012. 100(6): p. 1610-1620. 
16. Van Leeuwen, A.C., H. Yuan, G. Passanisi, J.W. van der Meer, J.D. de Bruijn, T.G. van Kooten, D.W. Grijpma, and R.R. Bos, Poly(trimethylene carbonate) and biphasic calcium phosphate composites for orbital floor reconstruction: a feasibility study in sheep. European Cells \& Materials, 2014. 27: p. 81-96.

17. Melchels, F.P., A.M. Barradas, C.A. van Blitterswijk, J. de Boer, J. Feijen, and D.W. Grijpma, Effects of the architecture of tissue engineering scaffolds on cell seeding and culturing. Acta Biomaterialia, 2010. 6(11): p. 4208-4217.

18. Elomaa, L., A. Kokkari, T. Narhi, and J.V. Seppala, Porous 3D modeled scaffolds of bioactive glass and photocrosslinkable poly(epsilon-caprolactone) by stereolithography. Composites Science and Technology, 2013. 74: p. 99-106.

19. Schuller-Ravoo, S., S.M. Teixeira, J. Feijen, D.W. Grijpma, and A.A. Poot, Flexible and elastic scaffolds for cartilage tissue engineering prepared by stereolithography using poly(trimethylene carbonate)-based resins. Macromolecular bioscience, 2013. 13(12): p. 1711-1719.

20. Lee, J.W., G. Ahn, D.S. Kim, and D.W. Cho, Development of nano- and microscale composite $3 D$ scaffolds using PPF/DEF-HA and microstereolithography. Microelectronic Engineering, 2009. 86(4-6): p. 1465-1467.

21. Tanodekaew, S., S. Channasanon, P. Kaewkong, and P. Uppanan, PLA-HA scaffolds: preparation and bioactivity. 3rd International Conference on Tissue Engineering, 2013. 59: p. 144-149.

22. Popov, V.K., A.V. Evseev, A.L. Ivanov, V.V. Roginski, A.I. Volozhin, and S.M. Howdle, Laser stereolithography and supercritical fluid processing for customdesigned implant fabrication. Journal of materials science. Materials in medicine, 2004. 15(2): p. 123-128.

23. Barbieri, D., A.J. Renard, J.D. de Bruijn, and H. Yuan, Heterotopic bone formation by nano-apatite containing poly(D,L-lactide) composites. European Cells \& Materials, 2010. 19: p. 252-261.

24. Barbieri, D., H.P. Yuan, X.M. Luo, S. Fare, D.W. Grijpma, and J.D. de Bruijn, Influence of polymer molecular weight in osteoinductive composites for bone tissue regeneration. Acta Biomaterialia, 2013. 9(12): p. 9401-9413.

25. Chapter 3 of this thesis; Geven, M.A., D. Barbieri, H. Yuan, J.D. de Bruijn, and D.W. Grijpma, Preparation and mechanical properties of photo-crosslinked poly(trimethylene carbonate) and nano-hydroxyapatite composites. Clinical hemorheology and microcirculation, 2015. 60(1): p. 3-11.

26. Premachandra, J.K. and J.E. Mark, Effects of dilution during crosslinking on strain-induced crystallization in cis-1,4-polyisoprene networks. I. Experimental results. Journal of Macromolecular Science-Pure and Applied Chemistry, 2002. 39(4): p. 287-300.

27. Vasiliev, V.G., L.Z. Rogovina, and G.L. Slonimsky, Dependence of Properties of Swollen and Dry Polymer Networks on the Conditions of Their Formation in Solution. Polymer, 1985. 26(11): p. 1667-1676. 


\section{Chapter 4}

28. Urayama, K. and S. Kohjiya, Uniaxial elongation of deswollen polydimethylsiloxane networks with supercoiled structure. Polymer, 1997. 38(4): p. $955-962$. 


\title{
Chapter 5 - The in vitro degradation of photo-crosslinked poly(trimethylene carbonate) and nano-hydroxyapatite composites
}

Mike A. Geven, Zhengchao Guo and Dirk W. Grijpma

Department of Biomaterials Science and Technology, University of Twente, Enschede, The Netherlands

\begin{abstract}
Composites of photo-crosslinked poly(trimethylene carbonate) and nano-hydroxyapatite are effective materials for the restoration of bone defects. The degradation characteristics of these composites have not yet been reported, however. We have prepared photocrosslinked poly(trimethylene carbonate) and composites containing 20.8 and $42.3 \mathrm{wt} . \%$ of nano-hydroxyapatite. To investigate the effect of the medium and nano-hydroxyapatite content on the in vitro degradation of the composites, these materials were incubated in aqueous media with neutral- or acidic $\mathrm{pH}$, or in solutions containing enzymes (cholesterol esterase or lipase). Changes in apparent mechanical properties, surface morphology, water uptake, mass loss and composition were followed using scanning electron microscopy, gravimetry and thermogravimetry. Even though photo-crosslinked poly(trimethylene carbonate) without nano-hydroxyapatite was nearly unaffected by all degradation media, the composites were found to have degraded in the enzyme solutions and in the acidic medium. The media were nearly selective for either of the two composite phases however, resulting in changes in the composition during their incubation. After 52 weeks in lipase solution, the composites with an initial nano-hydroxyapatite content of 20.8 and $42.3 \mathrm{wt} \%$ lost $88 \%$ and $87 \%$ of their photo-crosslinked poly(trimethylene carbonate) and only $8 \%$ and $12 \%$ of their nano-hydroxyapatite, respectively. This resulted in very brittle composites which were enriched with nano-hydroxyapatite. In contrast, composites with an initial nano-hydroxyapatite content of 20.8 and $42.3 \mathrm{wt} . \%$ incubated in acidic medium lost only $2 \%$ and $13 \%$ of their photo-crosslinked poly(trimethylene carbonate) and $19 \%$ and $66 \%$ of their nano-hydroxyapatite respectively. This resulted in more flexible composites with surfaces depleted of nano-hydroxyapatite. The results show that the nano-hydroxyapatite component significantly enhances the degradation of the composites and that they may be completely resorbable in vivo in a bony defect site.
\end{abstract}




\section{Chapter 5}

\section{Introduction}

Implants for bone tissue regeneration, based on composites of polymers and inorganics such as calcium phosphates or bioactive glass have gained increasing attention over the past decades. The use of composites increasingly gained preference over neat polymeric materials, as their preparation is an attractive method to produce materials with suitable mechanical properties as well as to imbue osteoconductive or-inductive properties[1-3].

Composites for use in bone tissue restoration frequently consist of biodegradable poly(lactic acids), poly(glycolic acid) or poly( $\varepsilon$-caprolactone) (PCL) combined with calcium phosphates or bioactive glass. The use of such biodegradable components allows for replacement of the implanted composite by newly formed bone over time without the need for additional surgical intervention. The characteristics of their degradation such as the type (bulk degradation or surface erosion), rate and compositional changes are important for successful reconstruction of a bony defect[2].

Reports that specifically discuss the degradation of composites of biodegradable polymers and inorganic fillers have focused on abovementioned polyester-based composites. It has been shown that poly(L-lactic acid), poly(D,L-lactic acid) (PDLLA), poly(lactic-coglycolic acid) (PLGA) and PCL composites with hydroxyapatite (HA), bioactive glass or akermite $\left(\mathrm{Ca}_{2} \mathrm{MgSi}_{2} \mathrm{O}_{7}\right)$ degrade more rapidly with increasing inorganic filler content[414]. This is generally explained by an enhanced water permeation into the composites and the enlarged polymer/filler interface, which promotes hydrolytic chain scission of the polymer[4, 5]. However, in the case of PDLLA and PLGA composites, it has been shown that the autocatalysis of the degradation reaction by the degradation products is reduced due to the buffering effect of the dissolution products of the inorganic filler[6, 8,9]. Due to the slow degradation rate of PCL in aqueous medium, the enhanced degradation rate of its composites with increasing filler content was also ascribed to an enhanced dissolution rate of the filler[11-14]. For other polyester-based composites containing poly(hydroxybutyrate), poly( $\varepsilon$-caprolactone-co-D,L-lactide) copolymers or poly(propylene fumarate) it was also found that an increasing filler content enhances degradation rates[1517].

Although polyester-based composites and their degradation have been investigated extensively, composites of biodegradable polycarbonates have been overlooked. A biodegradable polycarbonate that shows promising results for the use in biomedical applications like bone tissue regeneration is poly(trimethylene carbonate) (PTMC). In our previous work, we have shown that composites of photo-crosslinked PTMC and nano-HA (nHA) are suitable materials for bone reconstruction and promising results were obtained in vitro and in vivo in small animal models[18-20]. The degradation of PTMC has been investigated before and it was shown that it degrades via an enzymatic and oxidative pathway and is stable against non-enzymatic hydrolysis[21-24]. Furthermore, PTMC is stable over a wide $\mathrm{pH}$ range (1 to 13) and mainly PTMC oligomers along with 1,3-propane diol and trimethylene carbonate are formed during enzymatic degradation[21]. Degradation of photo-crosslinked PTMC networks has been investigated as well and was determined to 
proceed slowly in vivo and in vitro in enzyme solutions, with a dependence on the crosslinking density[22, 25]. The degradation of photo-crosslinked composite materials based on PTMC was not yet investigated, however. In this work we report on the in vitro degradation of composites consisting of photo-crosslinked PTMC and nHA. We have investigated degradation under neutral- and acidic conditions and in enzyme mediated degradation processes. We show the mass loss of the composites over time in combination with compositional, thermal and topographical changes. This gives insight into the fate of such composites during long-term in vivo application. Additionally it will yield insights on the effect of hydrophilic filler content on degradation of PTMC-based composites in general.

\section{Experimental}

\subsection{Materials}

Huizhou ForYou Medical Devices Ltd. provided trimethylene carbonate (TMC) monomer. A nHA powder with needle-like HA particles of 200 to $400 \mathrm{~nm}$ long and 20 to $50 \mathrm{~nm}$ wide was kindly provided by Kuros BioSciences BV. Tin(II) 2-ethylhexanoate ( $\left.\operatorname{Sn}(\mathrm{Oct})_{2}\right), 1,1,1$ tris(hydroxymethyl)propane (TMP), hydroquinone, methacrylic anhydride, triethylamine, deuterated chloroform, phosphate buffered saline (PBS) tablets ( $\mathrm{pH} 7.4$ ), cholesterol esterase powder ( $\mathrm{CE}$, from porcine pancreas, $35 \mathrm{U} / \mathrm{mg}$ ), lipase solution (from Themomyces lanuginosus, $\geq 100,000 \mathrm{U} / \mathrm{g})$, monobasic potassium phosphate $\left(\mathrm{KH}_{2} \mathrm{PO}_{4}\right)$ and sodium azide were acquired from Sigma Aldrich. Propylene carbonate and calcium hydride were from Merck Millipore. Omnirad TPO-L photo-initiator was provided by IMG Resins BV. Orasol Orange $G$ dye was acquired from Ciba Specialty Chemicals. Dichloromethane (DCM), methanol and chloroform were from VWR chemicals. The DCM was dried over calcium hydride and subsequently distilled under $\mathrm{N}_{2}$ atmosphere directly before use. Other chemicals were used as received unless stated otherwise.

\subsection{Methods}

\subsubsection{Synthesis of methacrylate end-group functionalized three-armed PTMC}

Methacrylate end-group functionalized three-armed PTMC (PTMC-MA) was synthesized as previously described[18]. Briefly, a three-armed PTMC oligomer was synthesized by ring-opening polymerization of TMC initiated by TMP in the presence of $\mathrm{Sn}(\mathrm{Oct})_{2}(0.13$ wt.\%) as catalyst. The ratio of TMC to TMP was 96.4 ( $\mathrm{mol} / \mathrm{mol})$. The ring-opening was performed at $130{ }^{\circ} \mathrm{C}$ for three days. The resulting PTMC oligomer was thereafter dissolved in dried DCM and $0.1 \mathrm{wt} . \%$ hydroquinone, triethylamine $(3 \mathrm{~mol} / \mathrm{mol}$ of PTMC hydroxyl end-groups) and methacrylic anhydride ( $3 \mathrm{~mol} / \mathrm{mol}$ of PTMC hydroxyl end-groups) were subsequently added while stirring. This functionalization reaction was conducted at room temperature for 5 days in the dark. The reaction mixture was then precipitated in ice-cooled methanol and the precipitate was additionally purified by extraction in ice-cooled methanol for 2 hours. It was thereafter dried in vacuo until constant weight. 
The molar mass and degree of functionalization of the PTMC-MA was determined by ${ }^{1} \mathrm{H}$ NMR in deuterated chloroform as was previously described[26]. A number averaged molar mass of $9.5 \mathrm{~kg} / \mathrm{mol}$ and a degree of functionalization of $93 \%$ was determined.

\subsubsection{Preparation of photo-crosslinked PTMC and composite films}

Resins containing 0, 9.1 and $16.7 \mathrm{wt} \% \mathrm{nHA}$ were produced for the preparation of composite films with 0,20 and 40 wt. $\%$ nHA respectively. A calculated amount of nHA was initially dispersed in chloroform and PTMC-MA was dissolved into the dispersion at $0.1 \mathrm{~g} / \mathrm{ml}$. Thereafter it was mechanically stirred while precipitating in ice-cooled methanol. The precipitate was dried in vacuo until constant weight and propylene carbonate was subsequently added while stirring at $60{ }^{\circ} \mathrm{C}$ in the dark. After three days the resulting resin was sonicated for 30 minutes at $60{ }^{\circ} \mathrm{C}$ to obtain a homogeneous dispersion. Afterwards, Orasol Orange G and Omnirad TPO-L were immediately dissolved in the dispersion to form the final resin. The composition of the resins is given in table 1.

Photo-crosslinked films with a designed thickness of $500 \mu \mathrm{m}$ were prepared in a layer-bylayer fashion by stereolithography using an EnvisionTec Perfactory3® SXGA ${ }^{+}$Standard UV system. The films were built up from 10 layers of $50 \mu \mathrm{m}$ each, with irradiation times of 9 seconds at $180 \mathrm{~mW} / \mathrm{dm}^{2}$ per layer. The resulting films were extracted in $1 / 1(\mathrm{v} / \mathrm{v})$ propylene carbonate/ethanol for three days, refreshing the extraction medium daily. The propylene carbonate diluent was subsequently removed by a stepwise exchange of the extraction medium to pure ethanol. Extracted films were dried at ambient conditions until constant weight.

Table 1. Composition of resins for photo-crosslinked PTMC and composite preparation.

\begin{tabular}{lccc}
\hline & \multicolumn{3}{c}{ Resins } \\
\cline { 2 - 4 } & PTMC-MA & PTMC-MA/nHA20 & PTMC-MA/nHA40 \\
\hline PTMC-MA (wt.\%) & 60 & 36.4 & 25 \\
nHA (wt.\%) & 0 & 9.1 & 16.7 \\
Propylene carbonate(wt.\%) & 40 & 54.5 & 58.3 \\
Orasol Orange G (wt.\%*) & 0.15 & 0.1 & 0.08 \\
Omnirad TPO-L (wt.\%*) & 5 & 5 & 5 \\
\hline
\end{tabular}

*Relative to PTMC-MA.

\subsubsection{In vitro degradation and sample analysis}

Samples for in vitro degradation were die-cut from the photo-crosslinked PTMC and composite films $(\varnothing=1 \mathrm{~cm})$. Their initial weight $\left(\mathrm{m}_{\mathrm{i}}\right)$ was recorded and they were thereafter submersed separately in $1 \mathrm{ml}$ of degradation medium. As degradation media, PBS, cholesterol esterase dissolved in PBS at $1 \mathrm{U} / \mathrm{ml}$, lipase solution and a $\mathrm{KH}_{2} \mathrm{PO}_{4}$ buffer solution in demineralized water at $\mathrm{pH} 4.5 \pm 0.05$ were used. To all degradation media, $0.02 \%$ $(w / v)$ of sodium azide was added to prevent bacterial growth during the degradation experiment. After submersion in degradation media, the samples were kept at $37^{\circ} \mathrm{C}$ and the 
media were refreshed twice per week. After 2, 4, 8, 16, 32 and 52 weeks, samples $(\mathrm{n}=3)$ were removed from the media and washed with demineralized water three times. The wet weight of the samples after blotting their surface $\left(\mathrm{m}_{\mathrm{w}}\right)$ was subsequently recorded. The samples were thereafter dried at $37^{\circ} \mathrm{C}$ for 3 weeks and afterwards lyophilized for 3 days. The dry weight of the samples after degradation $\left(\mathrm{m}_{\mathrm{d}}\right)$ was then recorded. The water uptake (WU in \%) of the samples and the percentage of mass remaining $\left(\mathrm{p}_{\mathrm{mr}}\right)$ were calculated according to equations 1 and 2 .

$$
\begin{gathered}
W U=\frac{m_{w}-m_{d}}{m_{d}} * 100 \% \\
p_{m r}=\frac{m_{d}}{m_{i}} * 100 \%
\end{gathered}
$$

The initial water uptake of samples before degradation was determined after incubation of samples $(n=3)$ of each film in demineralized water for 48 hours at $37{ }^{\circ} \mathrm{C}$. The $\mathrm{m}_{\mathrm{w}}$ and $\mathrm{m}_{\mathrm{d}}$ of these samples was thereafter determined and WU was calculated according to equation 1.

Mechanical properties of the samples were assessed qualitatively by manually bending the samples after incubation and drying. Apparent flexibility or brittleness of the samples was then compared to their initial state.

After drying, the surfaces of samples were imaged by SEM following sputter coating with chromium. A Zeiss Merlin scanning electron microscope with high efficiency secondary electron detector at 1.4 to $3 \mathrm{kV}$ was used. Samples for the determination of the initial water uptake before degradation were also used to obtain images of the sample surface before subjection to degradation media.

Thermogravimetrical analysis (TGA) was performed using a TGA7 from Perkin-Elmer to determine the nHA content and onset of thermal decomposition of samples. These were heated under $\mathrm{N}_{2}$-flow from 50 to $600{ }^{\circ} \mathrm{C}$ at a heating rate of $20{ }^{\circ} \mathrm{C} /$ minute. The percentage of each phase that was removed from the composite samples during in vitro degradation at a given time point ( $\mathrm{t}$ ) was calculated from the weight loss data and nHA content of samples as was determined by TGA. Equation 3 and 4 were used for these calculations.

$$
\begin{array}{r}
\Delta \varphi_{n H A}=\frac{f_{n H A, t} * p_{m r}}{f_{n H A, t=0}}-100 \% \\
\Delta \varphi_{P T M C}=\frac{\left(1-f_{n H A, t}\right) * p_{m r}}{1-f_{n H A, t=0}}-100 \%
\end{array}
$$

In equation 3 and $4, \Delta \varphi_{\mathrm{nHA}}$ is the percentage of nHA removed from the composite and $\Delta \varphi_{\text {PTMC }}$ is the percentage of photo-crosslinked PTMC removed from the composite, $\mathrm{f}_{\mathrm{nHA}, \mathrm{t}=0}$ is the weight fraction of nHA in the composites before incubation in degradation media and $\mathrm{f}_{\mathrm{nH}, \mathrm{t}}$ is the weight fraction of $\mathrm{nHA}$ at week $\mathrm{t}$ of degradation. 


\section{Results and Discussion}

\subsection{Initial sample characteristics}

The initial nHA content of the composites and water uptake of the photo-crosslinked PTMC and the composites was investigated before subjection to degradation media. From TGA it was determined that films prepared with PTMC-MA/nHA20 and PTMC$\mathrm{MA} / \mathrm{nHA} 40$ resins possessed an nHA content of $20.8 \pm 0.2$ and $42.3 \pm 0.5 \mathrm{wt}$. $\%$ respectively. These values are close to the intended nHA contents of the photo-crosslinked films. The water uptake of the photo-crosslinked PTMC and its composites with 20.8 and 42.3 wt.\% nHA was $1.3 \pm 0.6,3.7 \pm 0.2$ and $10.3 \pm 0.9 \%$ respectively. Thus, the composites became more hydrophilic with increasing nHA content as was expected from the hydrophilicity of nHA.

\subsection{Mechanical properties and surface morphology}

During the in vitro degradation of the photo-crosslinked PTMC and its composites with nHA, changes in the mechanical properties of the films could be observed qualitatively by manual bending of the specimens. These changes are correlated to the degradation of the samples along with compositional changes as we will show later in this work. An overview of the qualitative changes of the mechanical properties of the samples is offered in table 2. Samples that were incubated in PBS did not show any noticeable changes up to 52 weeks of incubation. Composites incubated in the enzyme solutions became brittle. This was more noticeable when using lipase compared to cholesterol esterase. Additionally, for samples with 42.3 wt. $\%$ nHA this was noticeable earlier than for samples with 20.8 wt. $\%$ nHA. In the case of samples with 42.3 wt.\% nHA incubated in lipase solution, samples fractured after 16 weeks. Photo-crosslinked PTMC without nHA was not greatly affected by the enzymes, although in lipase solution the stiffness was found to have been reduced after 4 weeks. When incubated in acidic $\mathrm{KH}_{2} \mathrm{PO}_{4}$ buffer solution, the stiffness of all samples was reduced. This was slightly delayed when $42.3 \mathrm{wt}$.\% nHA was incorporated compared to the other sample types. 


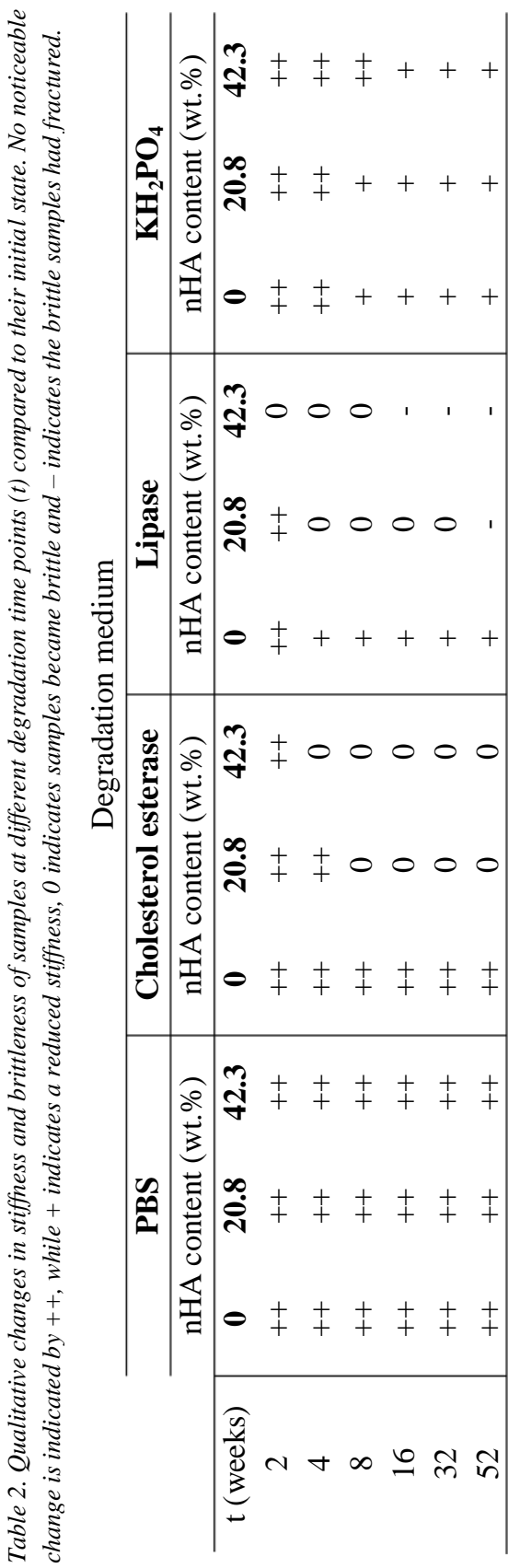


The surfaces of the photo-crosslinked PTMC and the composites were monitored using SEM and are shown in figure 1.

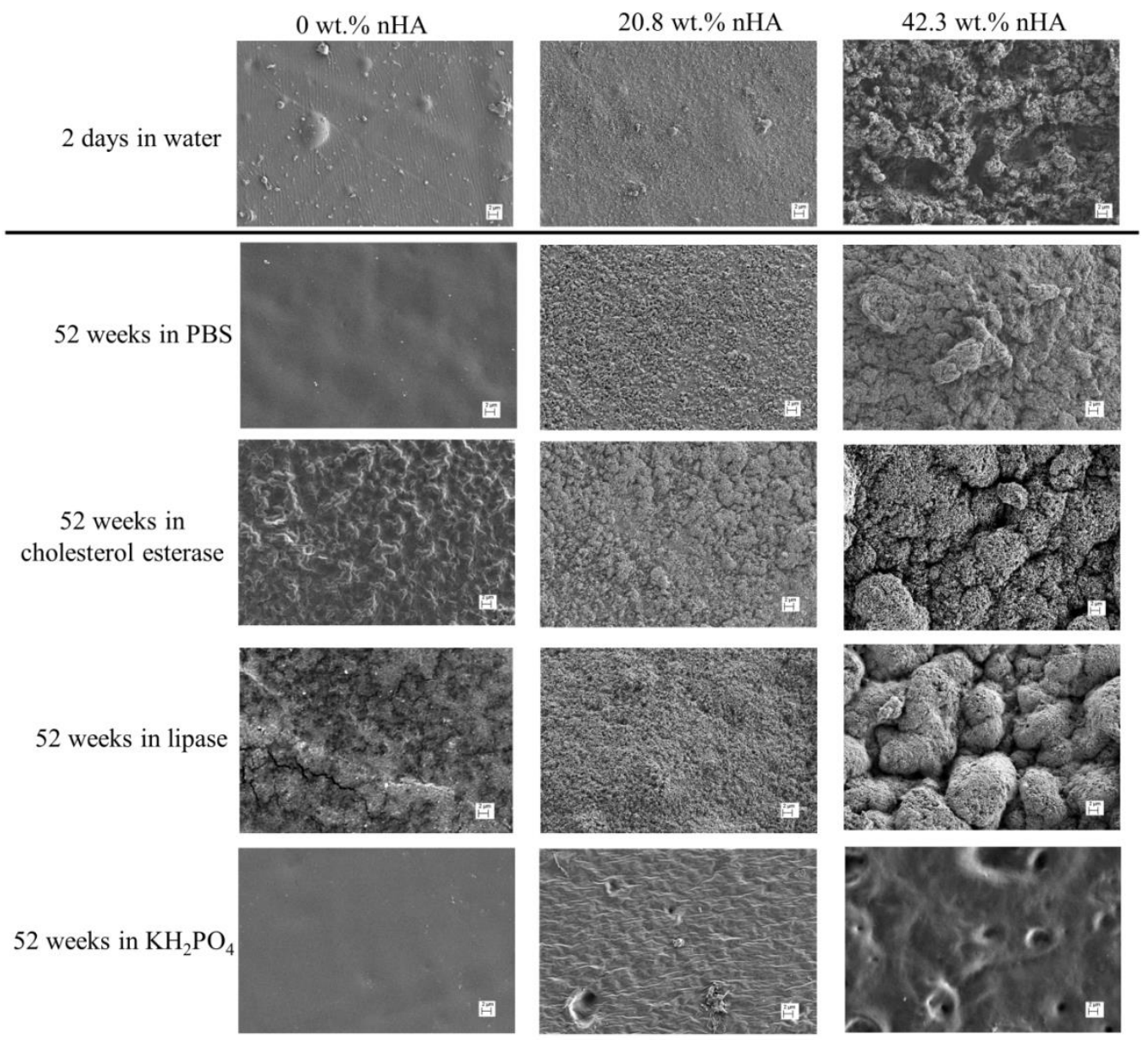

Figure 1. SEM images of composite surfaces after incubation in water as control before degradation and after 52 weeks of incubation in the different degradation media. Scale bars are $2 \mu \mathrm{m}$.

Figure 1 shows the surface structure of photo-crosslinked PTMC and composites before degradation and after 52 weeks in the different degradation media. Changes of the surfaces of photo-crosslinked PTMC were observed only after incubation in the enzyme solutions in which the surfaces became rougher. In PBS or $\mathrm{KH}_{2} \mathrm{PO}_{4}$ buffer, photo-crosslinked PTMC surfaces remained relatively smooth. The composites with an initial nHA content of 20.8 wt.\% were rougher with nHA particles protruding from the surface of the composite. The composites with initially 42.3 wt.\% nHA possessed a highly rough surface morphology with several nHA agglomerates amidst photo-crosslinked PTMC. After incubation in PBS, 
no significant difference between the composites before and after incubation was observed. Following incubation in both enzyme solutions, the surfaces of both composites were lacking photo-crosslinked PTMC in between the nHA particles. This was most clear for the composites with initially 20.8 wt. $\%$ nHA due to the higher initial content of polymer network. Following incubation in $\mathrm{KH}_{2} \mathrm{PO}_{4}$ buffer solution the composite surfaces were deprived of nHA. On the composites with initially 20.8 wt.\% nHA only few particles were observed on the surface of the composite. Some agglomerates of nHA were visible on the composites with initially $42.3 \mathrm{wt} . \% \mathrm{nHA}$, alongside several pits where agglomerates were initially present.

\subsection{Changes in water uptake and remaining mass}

The water uptake and remaining mass of photo-crosslinked PTMC and composites after incubation in the different degradation media was determined gravimetrically. Both are depicted in figure 2.

Photo-crosslinked PTMC films without nHA take up small amounts of water. Only after incubation in lipase solution the water uptake increased, up to 7\%. Similarly, the remaining mass of the photo-crosslinked PTMC samples only changed significantly in lipase solution. In this medium a continuous mass loss was observed and the remaining mass was $94.7 \%$ after 52 weeks of incubation.

The composites with an initial nHA content of $20.8 \mathrm{wt} . \%$ were more strongly affected by the degradation media. The water uptake was enhanced in all media and most notably increased to $18 \%$ and $96 \%$ in cholesterol esterase and lipase solution respectively. For this composite a slight mass loss was additionally observed in all media and in cholesterol esterase and lipase solution the remaining mass after 52 weeks was $87 \%$ and $28 \%$ respectively.

Composites with an initial nHA content of 42.3 wt.\% showed similar behavior in the different degradation media, although enhanced compared to composites with an initial nHA content of $20.8 \mathrm{wt} . \%$. In all media the water uptake initially increased up to 4 or 8 weeks incubation and thereafter decreased. Water uptake after 52 weeks incubation in cholesterol esterase and lipase solution most notably increased to $47 \%$ and $76 \%$ respectively. The mass loss of the samples with an initial nHA content of $42.3 \mathrm{wt} . \%$ was substantial in all degradation media. After 52 weeks incubation in PBS, cholesterol esterase solution, lipase solution and $\mathrm{KH}_{2} \mathrm{PO}_{4}$ buffer the remaining mass was $92 \%, 75 \%, 45 \%$ and $64 \%$ respectively. Only in lipase and $\mathrm{KH}_{2} \mathrm{PO}_{4}$ buffer solution, the mass loss was continuous however. 

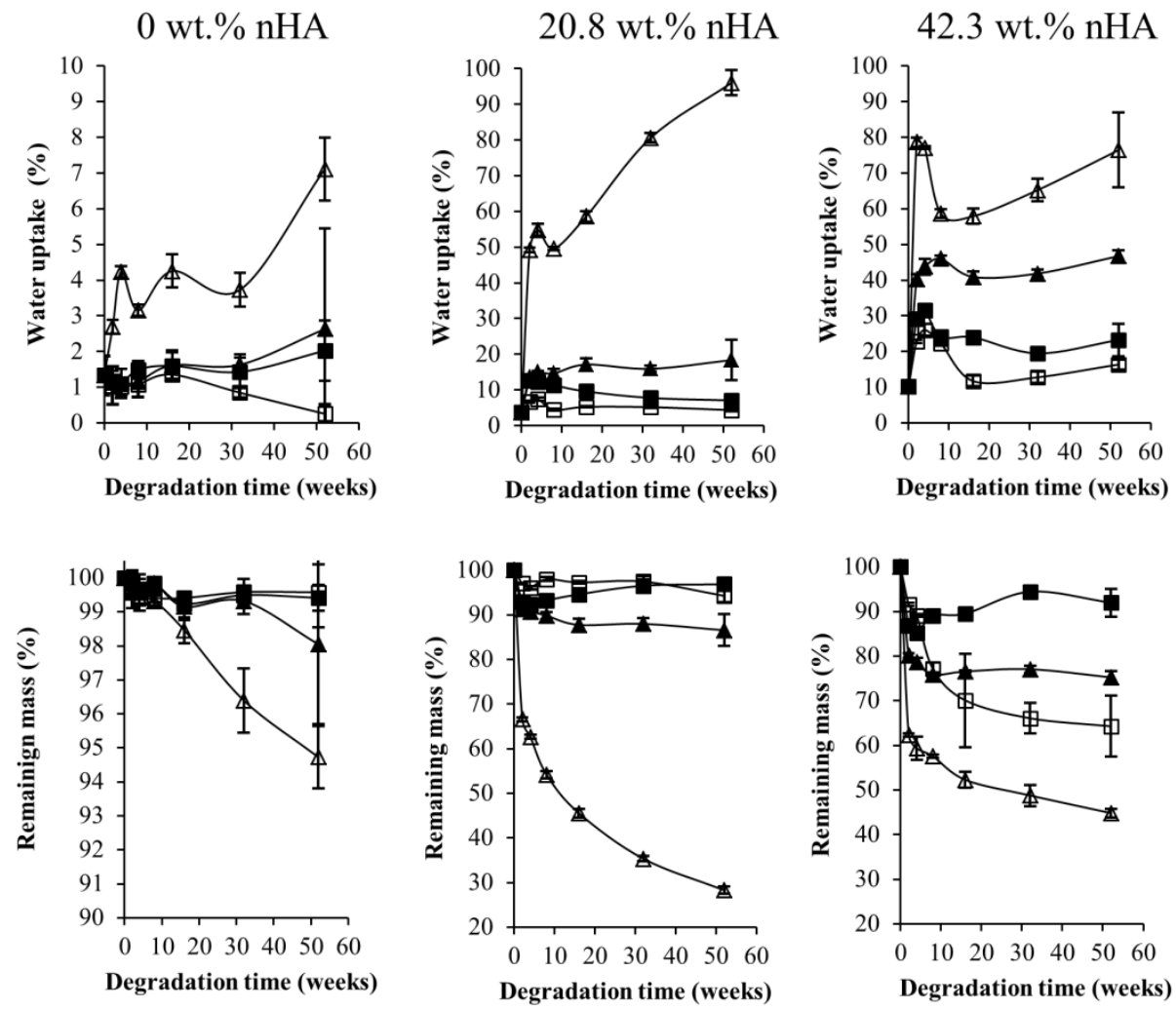

Figure 2. Water uptake and remaining mass of degradation samples after incubation in PBS (closed squares), cholesterol esterase solution (closed triangles), lipase solution (open triangles) and $\mathrm{KH}_{2} \mathrm{PO}_{4}$ buffer solution (open squares) (lines are drawn for the purpose of guiding the eye).

\subsection{Changes in composition}

The mass loss of the composites observed during the degradation experiment may be accompanied by compositional changes. These were investigated by TGA. Figure 3 shows the nHA content of the composites and the decomposition temperature of all materials throughout the degradation experiment.

As is shown in figure 3, the decomposition temperature of the photo-crosslinked PTMC was initially $312 \pm 5{ }^{\circ} \mathrm{C}$. During incubation in any of the degradation media, no significant change of the decomposition temperature was observed up to 16 weeks. Between 16 and 52 weeks of incubation, a decrease was observed which may be due to formation of dangling PTMC chains in the photo-crosslinked network during incubation in aqueous medium[27]. For composites with an initial nHA content of $20.8 \mathrm{wt} \%$ a slight increase in the nHA content was observed after incubation in cholesterol esterase, whereas a small decrease was observed in $\mathrm{KH}_{2} \mathrm{PO}_{4}$ buffer solution. In lipase solution, a strong increase of the nHA content was observed which was continuous up to 66 wt.\% after 52 weeks. This clearly 
causes these composites to become more brittle after degradation in the enzyme solutions. The degradation temperature of this composite was initially $273 \pm 4{ }^{\circ} \mathrm{C}$. For polyester-based composites with HA, the reduction of the decomposition temperature of polymer matrix in composites compared to the pristine polymer matrix has been described in literature before. This effect was ascribed to the catalysis of thermal decomposition of the ester bonds by HA[28]. Similarly, nHA may catalyze the decomposition of the carbonate bonds in the photo-crosslinked PTMC in the composites. By infrared spectroscopy we were able to show that there was indeed a close interaction between the photo-crosslinked PTMC and nHA in the composites before incubation in the degradation media. The infrared spectroscopy results are given in the supplementary information of this work. No significant changes of the decomposition temperature of composites with an initial nHA content 20.8 wt.\% were observed after incubation in PBS, cholesterol esterase solution or $\mathrm{KH}_{2} \mathrm{PO}_{4}$ buffer solution apart from a decrease between 16 and 52 weeks. After 2 weeks incubation in lipase solution, the decomposition temperature increased to a level close to that of photo-crosslinked PTMC however.

$$
0 \text { wt. } \% \text { nHA }
$$
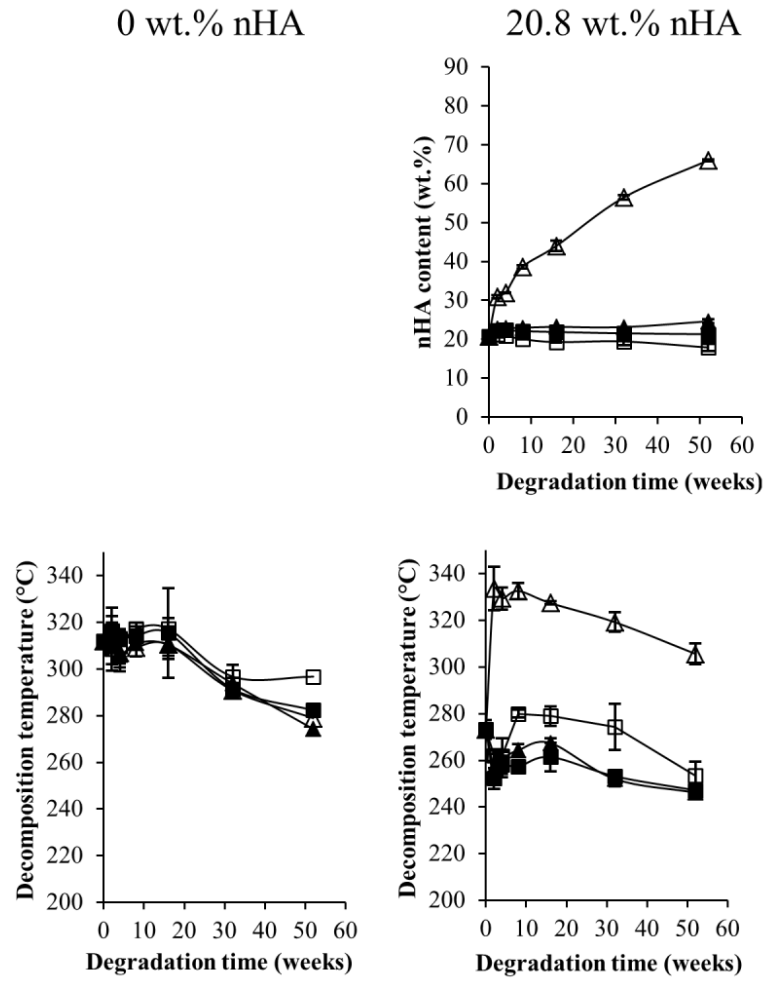

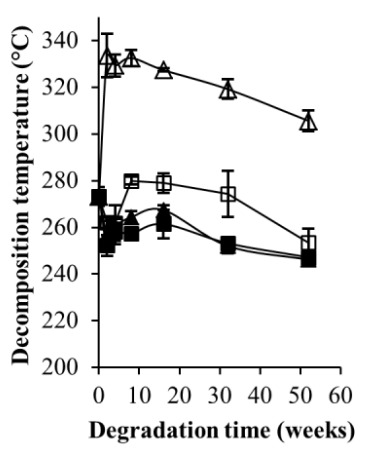

42.3 wt. $\%$ nHA
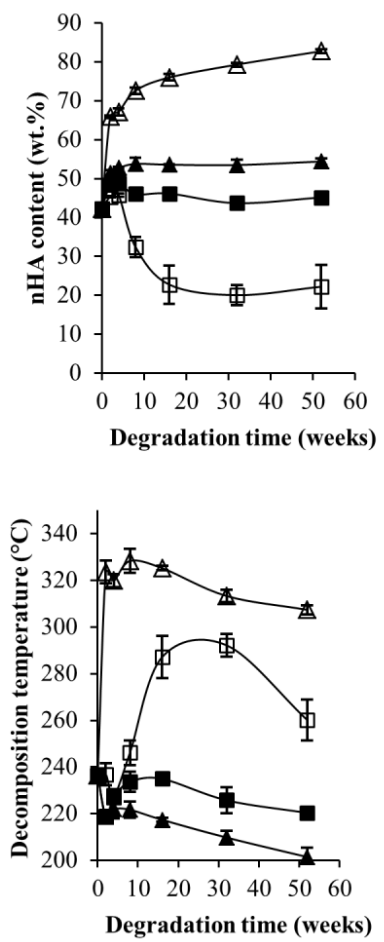

Figure 3. The nHA content and decomposition temperature of samples incubated in PBS (closed squares), cholesterol esterase solution (closed triangles), lipase solution (open triangles) and $\mathrm{KH}_{2} \mathrm{PO}_{4}$ buffer solution (open squares) (lines are depicted to guide the eye). 
Composites with an initial nHA content of 42.3 wt.\% all showed an initial increase of the nHA content. After 52 weeks of incubation in PBS no significant difference was found compared to the composite before incubation however. In cholesterol esterase solution the nHA content reaches a plateau value of 54 wt.\% after 8 weeks. In lipase solution the filler content continuously increased over time, reaching a value of 83 wt. $\%$ after 52 weeks. The filler content decreased in the acidic $\mathrm{KH}_{2} \mathrm{PO}_{4}$ buffer and was 22 wt.\% after 52 weeks. The initial decomposition temperature of this composite was $237 \pm 2{ }^{\circ} \mathrm{C}$. It was not significantly affected after incubation in PBS or cholesterol esterase solution apart from a decrease between 16 and 52 weeks. After incubation in lipase solution an increase of the decomposition temperature was observed after 2 weeks and a gradual increase of the decomposition temperature was observed after incubation in $\mathrm{KH}_{2} \mathrm{PO}_{4}$ buffer solution over 32 weeks. A subsequent decrease was observed, which may be due to formation of dangling chain ends. The initial increase of decomposition temperature of the composite indicates a reduced catalytic effect of the nHA during thermal decomposition. This could be due to a reduction in the contact area between the photo-crosslinked PTMC phase and the nHA phase over time as either is degraded or dissolved.

By combining mass loss and nHA content determined by gravimetry and thermogravimetry, the removal of each phase from the composites over time was calculated. The results of these calculations are depicted in figure 4, where the percentual changes of the polymer- and the nHA phases present in the composite are shown.

Figure 4 shows that the different degradation media are near specific for either of the two phases in the composites. In case of the composite with an initial nHA content of 20.8 wt.\%, the photo-crosslinked PTMC phase is significantly affected by the enzyme solutions. A gradual loss of this phase up to $18 \%$ is observed after 52 weeks of incubation in cholesterol esterase. In lipase solution up to $88 \%$ loss of the photo-crosslinked PTMC phase is observed. Concurrently, slight losses of nHA are observed in the enzyme solutions $(\leq 10 \%)$ as nHA is stable at neutral $\mathrm{pH}[29]$. In the acidic $\mathrm{KH}_{2} \mathrm{PO}_{4}$ buffer solution no loss of the photo-crosslinked PTMC phase was observed whereas a loss of nHA was observed up to $19 \%$ over 52 weeks. In PBS, the loss of both phases was minor $(\leq 4 \%)$.

Changes in the content of either phases in the composite with an initial nHA content of 42.3 wt.\% were enhanced compared to the composite with an initial nHA content of 20.3 wt. $\%$. After 52 weeks of incubation the loss of photo-crosslinked PTMC was $41 \%$ and $87 \%$ in cholesterol esterase and lipase solution respectively. In lipase solution a loss of nHA was also observed, which was $12 \%$ after 52 weeks. This may be due to dislodging of nHA aggregates as the loss of photo-crosslinked PTMC from this composite is high. In $\mathrm{KH}_{2} \mathrm{PO}_{4}$ buffer solution, the loss of nHA reached $66 \%$ and a loss of photo-crosslinked PTMC (13\%) was additionally observed after 52 weeks of incubation in this buffer. The large loss of nHA was expected from the $\mathrm{pH}$ susceptibility of this calcium phosphate. It is known that nHA is solubilized in vivo in the osteoclastic micro-environment at a $\mathrm{pH}$ of $4.5[29,30]$. 

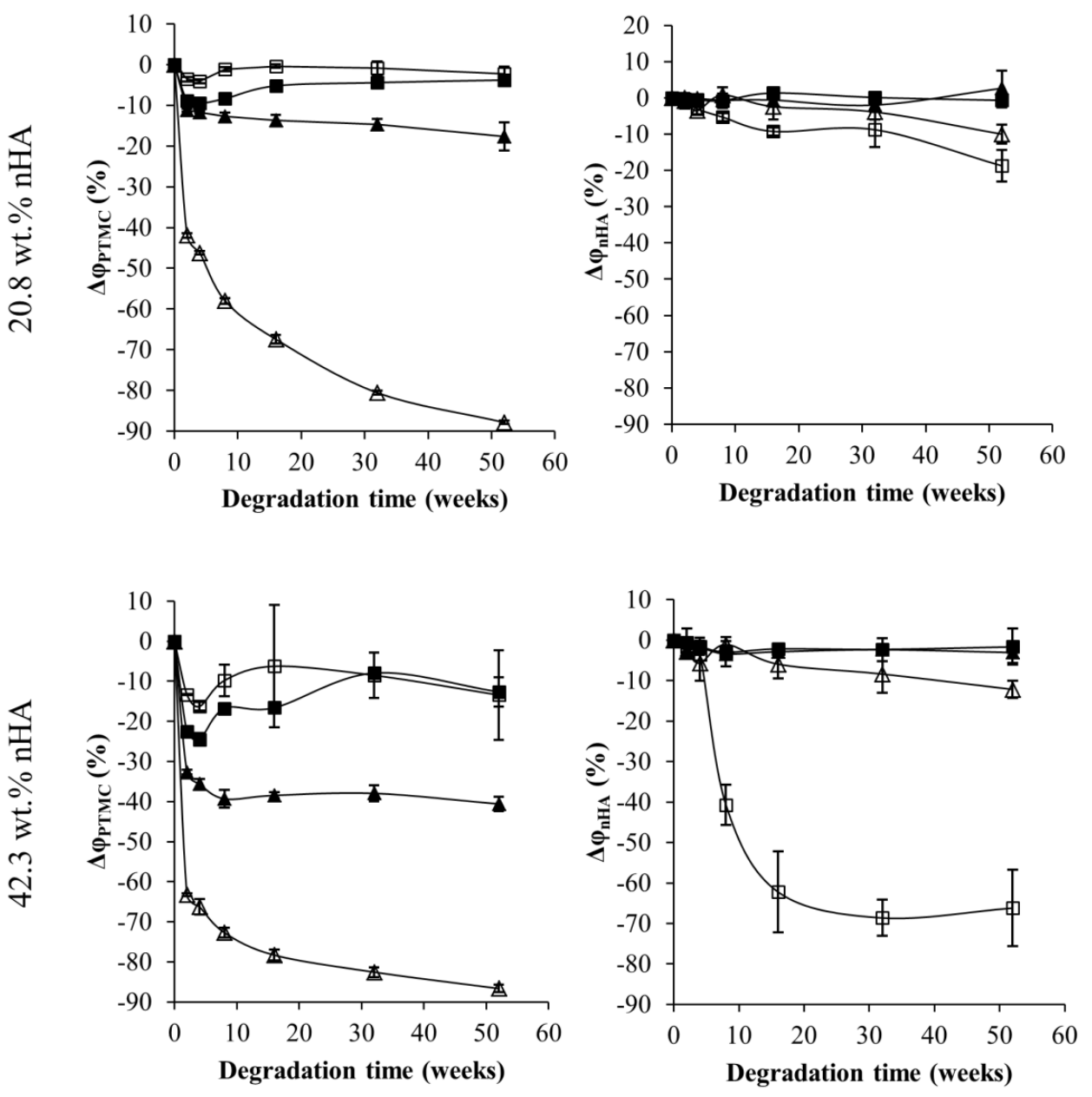

Figure 4. The percentages of photo-crosslinked PTMC or $n H A$ removed from composites incubated in PBS (closed squares), cholesterol esterase solution (closed triangles), lipase solution (open triangles) and $\mathrm{KH}_{2} \mathrm{PO}_{4}$ buffer solution (open squares). Photo-crosslinked PTMC removed from the composites is shown in the graphs on the left. The nHA removed from the composites is shown in the graphs on the right (lines are drawn to guide the eye).

The large losses of photo-crosslinked PTMC from composites in the enzyme solutions may be a result of the enhanced water uptake of the composites compared to photo-crosslinked PTMC without nHA. Due to the presence of nHA in photo-crosslinked PTMC, the enzyme solutions are able to penetrate into the bulk of the composites. It is likely that the enzymes consequently act at the interface of the nHA and photo-crosslinked PTMC. Hence, the nHA particles effectively enhance the surface area of photo-crosslinked PTMC that is accessible by cholesterol esterase or lipase in the composites compared to photo-crosslinked PTMC without nHA. As the photo-crosslinked PTMC is degraded, water uptake of the composites 
remains high (figure 2), and the degradation proceeds although the rate decreases over time. The enhanced dissolution of nHA with increasing nHA content in the composites in $\mathrm{KH}_{2} \mathrm{PO}_{4}$ buffer is a result of the higher initial water uptake as well as an improved interconnectivity between the nHA particles[18].

Taking into account the degradation and dissolution of both phases in the composites presented here, it is clear that incorporation of nHA into photo-crosslinked PTMC can control the degradation behavior of the resulting composite. Inclusion of nHA results in enhanced susceptibility of the photo-crosslinked PTMC to enzymes that are naturally present in macrophages[31]. Increasing the nHA content of the composites thereupon augments the dissolution rate of nHA in the acidic osteoclast environment that is naturally present in osseous sites. The nHA content can therefore also be used to control the degradation of the photo-crosslinked PTMC and nHA composites in vivo, and the composites will thus likely be completely resorbable.

\section{Conclusions}

Photo-crosslinked PTMC and its composites with nHA were subjected to different degradation media in vitro to investigate the effect of the degradation media and $\mathrm{nHA}$ particle content on degradation of the composite. In aqueous medium at neutral $\mathrm{pH}$, neither the photo-crosslinked PTMC nor its composites were strongly degraded. In enzyme solutions, a clear effect was observed on the composites, however. The composites became more brittle over time as a result of the degradation of the photo-crosslinked PTMC phase. The degradation of this phase became more prominent due to the enhanced water permeation into the composite and an enhanced surface area of the polymer phase that is available to enzymatic degradation. The nHA phase in the composites was mainly affected by acidic $\mathrm{KH}_{2} \mathrm{PO}_{4}$ buffer solution whereas the polymer network phase was relatively unaffected. This resulted in composites with lower stiffness. In both enzyme solutions and the $\mathrm{KH}_{2} \mathrm{PO}_{4}$ buffer solution, the degradation of the composites is enhanced with increasing nHA content. These results show that the nHA content may indeed be used to control the degradation of photo-crosslinked PTMC and nHA composites. Furthermore, enzymes and acidic conditions may completely degrade these composites in an in vivo situation as a result of the action of inflammatory cells as well as osteoclasts.

\section{References}

1. Kolk, A., J. Handschel, W. Drescher, D. Rothamel, F. Kloss, M. Blessmann, M. Heiland, K.D. Wolff, and R. Smeets, Current trends and future perspectives of bone substitute materials - from space holders to innovative biomaterials. Journal of Craniomaxillofacial Surgery, 2012. 40(8): p. 706-718.

2. Bose, S., M. Roy, and A. Bandyopadhyay, Recent advances in bone tissue engineering scaffolds. Trends in Biotechnology, 2012. 30(10): p. 546-554. 
3. Kinoshita, Y. and H. Maeda, Recent developments of functional scaffolds for craniomaxillofacial bone tissue engineering applications. ScientificWorldJournal, 2013. 2013: p. 863157.

4. Shikinami, Y. and M. Okuno, Bioresorbable devices made of forged composites of hydroxyapatite (HA) particles and poly-L-lactide (PLLA): Part I. Basic characteristics. Biomaterials, 1999. 20(9): p. 859-877.

5. Lin, P.L., H.W. Fang, T. Tseng, and W.H. Lee, Effects of hydroxyapatite dosage on mechanical and biological behaviors of polylactic acid composite materials. Materials Letters, 2007. 61(14-15): p. 3009-3013.

6. Barbieri, D., J.D. de Bruijn, X. Luo, S. Fare, D.W. Grijpma, and H. Yuan, Controlling dynamic mechanical properties and degradation of composites for bone regeneration by means of filler content. Journal of Mechanical Behavior of Biomedical Materials, 2013. 20: p. 162-172.

7. Russias, J., E. Saiz, R.K. Nalla, K. Gryn, R.O. Ritchie, and A.P. Tomsia, Fabrication and mechanical properties of PLA/HA composites: A study of in vitro degradation. Materials Science Engineering Part C - Biomimetic Supramolecular Systems, 2006. 26(8): p. 1289-1295.

8. Maquet, V., A.R. Boccaccini, L. Pravata, I. Nottingher, and R. Jérôme, Preparation, characterization, and in vitro degradation of bioresorbable and bioactive composites based on Bioglass ${ }^{\circledR}$-filled polylactide foams. Journal of Biomedical Materials Research, 2003. 66A: p. 335-346.

9. Maquet, V., A.R. Boccaccini, L. Pravata, I. Notingher, and R. Jerome, Porous poly(alpha-hydroxyacid)/Bioglass composite scaffolds for bone tissue engineering. I: Preparation and in vitro characterisation. Biomaterials, 2004. 25(18): p. 41854194.

10. Deng, Y., M. Zhang, X. Chen, X. Pu, X. Liao, Z. Huang, and G. Yin, A novel akermanite/poly (lactic-co-glycolic acid) porous composite scaffold fabricated via a solvent casting-particulate leaching method improved by solvent selfproliferating process. Regen Biomater, 2017. 4(4): p. 233-242.

11. Kim, H.W., J.C. Knowles, and H.E. Kim, Hydroxyapatite/poly(epsiloncaprolactone) composite coatings on hydroxyapatite porous bone scaffold for drug delivery. Biomaterials, 2004. 25(7-8): p. 1279-1287.

12. Ang, K.C., K.F. Leong, C.K. Chua, and M. Chandrasekaran, Compressive properties and degradability of poly(E-caprolatone)/hydroxyapatite composites under accelerated hydrolytic degradation. Journal of Biomedical Materials Research Part A, 2007. 80A(3): p. 655-660.

13. Wang, Y.Y., L. Liu, and S.R. Guo, Characterization of biodegradable and cytocompatible nano-hydroxyapatite/polycaprolactone porous scaffolds in degradation in vitro. Polymer Degradation and Stability, 2010. 95(2): p. 207-213.

14. Kouhi, M., M. Morshed, J. Varshosaz, and M.H. Fathi, Poly (epsiloncaprolactone) incorporated bioactive glass nanoparticles and simvastatin 
nanocomposite nanofibers: Preparation, characterization and in vitro drug release for bone regeneration applications. Chemical Engineering Journal, 2013. 228: p. 1057-1065.

15. Doyle, C., E.T. Tanner, and W. Bonfield, In vitro and in vivo evaluation of polyhydroxybutyrate and of polyhydroxybutyrate reinforced with hydroxyapatite. Biomaterials, 1991. 12(9): p. 841-847.

16. Rich, J., T. Jaakkola, T. Tirri, T. Narhi, A. Yli-Urpo, and J. Seppala, In vitro evaluation of poly(epsilon-caprolactone-co-DL-lactide)/ bioactive glass composites. Biomaterials, 2002. 23(10): p. 2143-50.

17. Jayabalan, M., K.T. Shalumon, M.K. Mitha, K. Ganesan, and M. Epple, Effect of hydroxyapatite on the biodegradation and biomechanical stability of polyester nanocomposites for orthopaedic applications. Acta Biomater, 2010. 6(3): p. 763775.

18. Chapter 4 of this thesis; Geven, M.A., V. Varjas, L. Kamer, X. Wang, J. Peng, D. Eglin, and D.W. Grijpma, Fabrication of patient specific composite orbital floor implants by stereolithography. Polymers for Advanced Technologies, 2015. 26(12): p. 1433-1438.

19. Chapter 6 of this thesis; Guillaume, O., M.A. Geven, D.W. Grijpma, T.T. Tang, L. Qin, Y.X. Lai, H. Yuan, R.G. Richards, and D. Eglin, Poly(trimethylene carbonate) and nano-hydroxyapatite porous scaffolds manufactured by stereolithography. Polymers for Advanced Technologies, 2017. 28(10): p. 12191225.

20. Chapter 7 of this thesis; Guillaume, O., M.A. Geven, C.M. Sprecher, V.A. Stadelmann, D.W. Grijpma, T.T. Tang, L. Qin, Y. Lai, M. Alini, J.D. de Bruijn, H. Yuan, R.G. Richards, and D. Eglin, Surface-enrichment with hydroxyapatite nanoparticles in stereolithography-fabricated composite polymer scaffolds promotes bone repair. Acta Biomaterialia, 2017. 54: p. 386-398.

21. Zhang, Z., R. Kuijer, S.K. Bulstra, D.W. Grijpma, and J. Feijen, The in vivo and in vitro degradation behavior of poly(trimethylene carbonate). Biomaterials, 2006. 27(9): p. 1741-1748.

22. Chapanian, R., M.Y. Tse, S.C. Pang, and B.G. Amsden, The role of oxidation and enzymatic hydrolysis on the in vivo degradation of trimethylene carbonate based photocrosslinkable elastomers. Biomaterials, 2009. 30(3): p. 295-306.

23. Jansen, J., S.A. Koopmans, L.I. Los, R.J. van der Worp, J.G. Podt, J.M. Hooymans, J. Feijen, and D.W. Grijpma, Intraocular degradation behavior of crosslinked and linear poly(trimethylene carbonate) and poly(D,L-lactic acid). Biomaterials, 2011. 32(22): p. 4994-5002.

24. Yang, L.Q., J.X. Li, W. Zhang, Y. Jin, J.Z. Zhang, Y. Liu, D.X. Yi, M. Li, J. Guo, and Z.W. Gu, The degradation of poly(trimethylene carbonate) implants: The role of molecular weight and enzymes. Polymer Degradation and Stability, 2015. 122: p. 77-87. 
25. Rongen, J.J., B. van Bochove, G. Hannink, D.W. Grijpma, and P. Buma, Degradation behavior of, and tissue response to photo-crosslinked poly(trimethylene carbonate) networks. Journal of Biomedical Materials Research Part A, 2016. 104(11): p. 2823-2832.

26. Chapter 3 of this thesis; Geven, M.A., D. Barbieri, H. Yuan, J.D. de Bruijn, and D.W. Grijpma, Preparation and mechanical properties of photo-crosslinked poly(trimethylene carbonate) and nano-hydroxyapatite composites. Clin Hemorheol Microcirc, 2015. 60(1): p. 3-11.

27. Yang, L.-Q., J. Li, W. Zhang, Y. Jin, J. Zhang, Y. Liu, D. Yi, M. Li, J. Guo, and $\mathrm{Z} . \mathrm{Gu}$, The degradation of poly(trimethylene carbonate) implants: The role of molecular weight and enzymes. Polymer Degradation and Stability, 2015. 122: p. 77-87.

28. Chen, D.Z., C.Y. Tang, K.C. Chan, C.P. Tsui, P.H.F. Yu, M.C.P. Leung, and P.S. Uskokovic, Dynamic mechanical properties and in vitro bioactivity of PHBHV/HA nanocomposite. Composites Science and Technology, 2007. 67(7): p. 1617-1626.

29. Silver, I.A., R.J. Murrills, and D.J. Etherington, Microelectrode studies on the acid microenvironment beneath adherent macrophages and osteoclasts. Experimental Cell Research, 1988. 175(2): p. 266-276.

30. Teitelbaum, S.L., Bone resorption by osteoclasts. Science, 2000. 289(5484): p. 1504-1508.

31. Azevedo, H. and R.L. Reis, Understanding the Enzymatic Degradation of Biodegradable Polymers and Strategies to Control Their Degradation Rate. 2005, Boca Raton, Fla, USA: CRC Press.

32. Nejati, E., H. Mirzadeh, and M. Zandi, Synthesis and Characterization of Nanohydroxyapatite Rods/poly (L-lactide acid) Composite Scaffolds for Bone Tissue Engineering. Vol. 39. 2008. 1589-1596. 


\section{Chapter 5 - Supplementary information}

Infrared spectroscopy was performed at the surface of the samples using attenuated total reflectance infrared (ATR-IR) spectroscopy. A Perkin-Elmer Spectrum Two was used between 450 and $4000 \mathrm{~cm}^{-1}$ with a spectral resolution of $0.5 \mathrm{~cm}^{-1}$.

The spectra of photo-crosslinked PTMC, nHA and both composite samples before incubation in media are given in figure S1. In table S1, relevant IR-peaks are given.

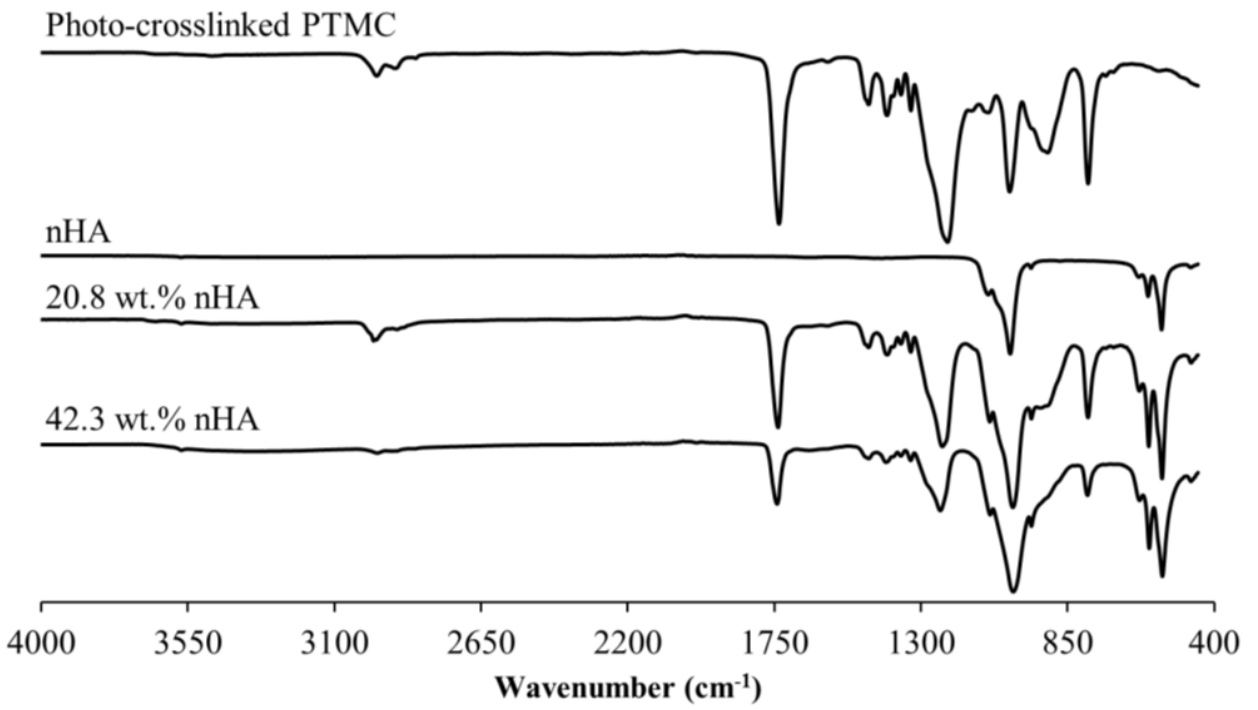

Figure S1. ATR-IR spectra of photo-crosslinked PTMC, nHA and composites with 20.8 and 42.3 wt. $\%$ nHA. 
Table S1. Relevant IR-peaks ( $\left.\mathrm{cm}^{-1}\right)$ of photo-crosslinked PTMC, $n$ HA and its composites with 20.8 and 42.3 wt.\% $n H A$.

\begin{tabular}{|c|c|c|c|c|}
\hline Material & $\begin{array}{c}\text { Photo- } \\
\text { crosslinked } \\
\text { PTMC }\end{array}$ & nHA & $\begin{array}{c}20.8 \text { wt. } \% \\
\text { nHA }\end{array}$ & $\begin{array}{c}42.3 \text { wt. } \% \\
\text { nHA }\end{array}$ \\
\hline $\mathrm{C}=\mathrm{O}$ & 1736 & - & 1739 & 1743 \\
\hline C-O & 1219 & - & 1235 & 1243 \\
\hline $\mathrm{CH}_{2}$ bending & 1404 & - & 1404 & 1408 \\
\hline $\mathrm{PO}_{4}{ }^{3-}$ & $\begin{array}{l}- \\
- \\
- \\
- \\
-\end{array}$ & $\begin{array}{c}562 \\
603 \\
962 \\
1026 \\
1094\end{array}$ & $\begin{array}{c}560 \\
601 \\
961 \\
1018 \\
1089\end{array}$ & $\begin{array}{c}560 \\
600 \\
961 \\
1022 \\
1089\end{array}$ \\
\hline $\mathbf{O H}$ & $\begin{array}{l}- \\
-\end{array}$ & $\begin{array}{c}632 \\
3572\end{array}$ & $\begin{array}{c}630 \\
3570\end{array}$ & $\begin{array}{c}630 \\
3571\end{array}$ \\
\hline
\end{tabular}

Figure $\mathrm{S} 1$ and table $\mathrm{S} 1$ show that the $\mathrm{C}=\mathrm{O}, \mathrm{C}-\mathrm{O}$ and $\mathrm{CH}_{2}$ bending peaks of photocrosslinked PTMC shift to higher wavenumbers with an increasing nHA content in the composites. The $\mathrm{PO}_{4}{ }^{3-}$ and $\mathrm{OH}$ peaks of nHA shift to lower wavenumbers in the composites. This is an indication of a close interaction between the PTMC oligomer chains in the photo-crosslinked network and the nHA particles, which is likely a dipole interaction between the PTMC carbonate groups and the phosphate groups of the nHA[32]. 


\title{
Chapter 6 - Poly(trimethylene carbonate) and nano- hydroxyapatite porous scaffolds manufactured by stereolithography
}

Olivier Guillaume ${ }^{\mathrm{a}}$, Mike A. Geven ${ }^{\mathrm{b}}$, Dirk W. Grijpma ${ }^{\mathrm{b}}$, Ting-Ting Tang ${ }^{\mathrm{c}}$, Ling Qin ${ }^{\mathrm{d}}$, Huipin Yuan ${ }^{\mathrm{e}}$, Robert G. Richards ${ }^{\mathrm{a}}$ and David Eglin ${ }^{\mathrm{a}}$

${ }^{\text {a }}$ AO Research Institute, Davos, Switzerland.

${ }^{\mathrm{b}}$ Department of Biomaterials Science and Technology, University of Twente, Enschede, The Netherlands.

${ }^{c}$ Shanghai Key Laboratory of Orthopaedic Implants, Department of Orthopaedic Surgery, Shanghai Ninth People's, Hospital, Shanghai Jiao Tong University School of Medicine, Shanghai, People's Republic of China.

${ }^{\mathrm{d}}$ Translational Medicine Research and Development Centre, Institute of Biomedical and Health Engineering, Shenzhen Institute of Advanced Technology, The Chinese Academy of Sciences, Shenzhen, People's Republic of China.

${ }^{\mathrm{e}}$ Kuros BioSciences BV, Bilthoven, The Netherlands.

\begin{abstract}
Designing calcium phosphate loaded polymeric porous scaffolds with controlled architecture using stereolithography (SLA) has great potential in the field of bone tissue engineering. However, use of poly(ester)s with suboptimal degradation property have mainly been reported. In the present work, we introduced a poly(trimethylene carbonate) (PTMC) and nano-hydroxyapatite (HA) resin suitable for SLA-manufacturing, and created cytocompatible 3D porous structure.

First, the resin formulation and HA content were optimized for photo-crosslink-based stereolithography fabrication process. Subsequently, we evaluated the influence of the resin composition on physico-chemical characteristics of fabricated films and scaffolds. Then, the influence of the biomaterial composition on hBMSCs viability, adhesion and proliferation was assessed.

Films and macro-porous scaffolds were successfully produced by photo-crosslinking with up to $40 \%$ weight of HA (relative to PTMC). We demonstrated that addition of HA in PTMC matrices induced a direct effect on the surface properties, in terms of wettability and topography. Additionally, mechanical tests revealed a correlation between the amounts of HA loaded in PTMC and the stiffness of the SLA-fabricated scaffolds. Importantly, the different photo-crosslinked biomaterials exhibited in vitro cytocompatibility, similar to tissue culture polystyrene. Those data suggest the possibility to fabricate highly tuneable HA loaded PTMC 3D porous structures for bone tissue engineering application.
\end{abstract}




\section{Introduction}

Medical application represents a fertile market for 3D printed devices, which is expected to reach a total business of $\$ 2$ billion in the next decade[1]. Additive manufacturing technology has appealing potential in the biomedical field, including bone tissue engineering[2]. Indeed, the driving force of these advanced manufacturing technologies, such as stereolithography (SLA), relies in the possibility to create customized implants and scaffolds with an unprecedented degree of resolution. This was recently illustrated by Schüller-Ravoo et al., who reproduced an anatomically-relevant microvasculature network, using SLA-approach[3]. Such complex and controlled architectures would be extremely challenging to achieve with other available techniques and machineries (e.g. gas forming, salt leaching, freeze-drying, etc.).

The utilization of SLA for the fabrication of biodegradable composite scaffolds combined with bioactive compounds is a relevant strategy in bone regenerative medicine[4]. Biological activity is commonly endowed to the light reactive based-polymeric resin by loading ceramic, proteins or stem cells either prior, for the former, or post fabrication for the latter's[5]. Biodegradable SLA-fabricated scaffolds have been produced using acrylate or methacrylate functional poly(ester) prepolymers, such as poly(propylene fumarate)[6], poly(e-caprolactone)[7] or poly(D,L-lactide)[8, 9] based-resins associated with various calcium phosphate $(\mathrm{CaP})$ ceramic particles to confer osteoconductivity to the fabricated scaffolds. Previous work related on SLA-fabricated PTMC scaffolds has shown that the fine control over their internal architecture, their compliancy and flexibility are advantageous for cells loading and infiltration, and for their stability in dynamic physiological conditions[10].

PTMC membrane has also been successfully used as a membrane guide for bone regeneration in rat mandibular defects. Although the amount of new bone formed was not significantly different compared to collagen and e-PTFE membranes, indicating the lack of significant osteoconductivity[11]. More recently, dense PTMC membranes were endowed with bioactivity by addition of $\beta$-tricalcium phosphate into PTMC matrices either by compression molding[12, 13] or by SLA[14, 15]. However, for bone tissue engineering approach, a certain degree of internal and interconnected porosity is mandatory to allow construct vascularization and osseointegration.

In consequence, the aim of this study was to manufacture macro-porous and composite scaffolds based on a poly(trimethylene carbonate) (PTMC) resin containing a high load of nano-hydroxyapatite (HA). Composite resin formulations based on methacrylated-PTMC were first optimized and two loads of HA used for the preparation of films and macroporous scaffolds by photo-crosslinking reaction and SLA respectively. The surface wettability and topography of the composites were characterized and compared to crosslinked PTMC film. Scaffolds were characterized for their shape retention, surface topography, HA content and elastic modulus. Finally, cytocompatibility and proliferation experiments using human bone marrow stromal cells were performed on surfaces of PTMC-HA compared to PTMC and optimized culture plastic surfaces. 


\section{Experimental section}

\subsection{Materials fabrication}

\subsubsection{Preparation of photo-crosslinkable PTMC}

Three-armed poly(trimethylene carbonate) (PTMC) was synthesized by ring-opening polymerization of trimethylene carbonate (TMC, received from Huizhou Foryou Medical Devices Co. Ltd., China) initiated by trimethylolpropane (from Fluka analytical). Briefly, in a molar ratio of 96.8/1, TMC/trimethylolpropane was loaded in a flask under dry $\mathrm{N}_{2}$ atmosphere. Using $0.13 \%$ wt. of Tin(II) ethylhexanoate ( $\mathrm{Sn}(\mathrm{Oct})_{2}$, from Sigma-Aldrich) as a catalyst, the polymerization was conducted at $130^{\circ} \mathrm{C}$ for 3 days. The formed PTMC with number average molecular weight of $10 \mathrm{~kg} / \mathrm{mol}$, as determined by ${ }^{1} \mathrm{H}-\mathrm{NMR}$ [14], was thereafter dissolved in dichloromethane under dry $\mathrm{N}_{2}$ atmosphere. In order to obtain a photo-crosslinkable compound, PTMC macromer with methacrylate end-groups (PTMCMA) was subsequently prepared by reacting PTMC hydroxyl groups with methacrylic anhydride (from Sigma-Aldrich), as recently described, in order to obtain a degree of functionalization of $99 \%$ [15].

\subsubsection{Fabrication of photo-crosslinked PTMC composite structures}

Resin formulations were prepared with gradual increase of nano-hydroxyapatite (HA, supplied by Kuros BioSciences BV, Bilthoven, The Netherlands) content in relation to PTMC-MA. Briefly, PTMC-MA and HA were dispersed in chloroform overnight under stirring, precipitated in cold methanol and dried in order to obtain homogenous powder. Subsequently, PTMC-MA HA powder was mixed with propylene carbonate diluent (Merck Millipore), Lucirin ${ }^{\circledR}$ TPO-L photo-initiator (BASF) and Orasol Orange G dye (from Ciba Speciality Chemicals).

Thin films were prepared by solvent casting of the resins on glass plates and subsequent photo-crosslinking in a UV-crosslinking box at $365 \mathrm{~nm}$ and $1100 \mathrm{~mW} / \mathrm{dm}^{2}$ for $5 \mathrm{~min}$ under $\mathrm{N}_{2}$. Following fabrication, propylene carbonate diluent was removed by extraction in propylene carbonate/ethanol (1/1 v/v), gradually exchanged to $100 \%$ ethanol and then the structures were dried under ambient conditions until constant weight. Obtained films were sterilized using cold ethylene oxide gas procedure and finally kept under vacuum for 5 days before use.

Scaffold (6 x $3.5 \mathrm{~mm}$, diameter $\mathrm{x}$ height) with gyroid porosity were fabricated using an Envisiontec Perfactory ${ }^{3} \circledR \mathrm{SXGA}^{+}$Standard UV stereolithography system. A light intensity of $180 \mathrm{~mW} / \mathrm{dm}^{2}$ was used with an irradiation time of 9 seconds per layer and the platform was elevated by $50 \mu \mathrm{m}$ between each layer. Following materials fabrication, the samples were processed as described for the films.

\subsection{Materials characterization}

The wettability of the different films was characterized using contact angle method (Drop Shape Analysis System, DSA10 Kruss), on samples sterilized (using EO) and dried beforehand (at $40^{\circ} \mathrm{C}$ under vacuum $200 \mathrm{mbar}$ for $48 \mathrm{hrs}$ ) (10 measurements per group). 
Additionally, the surface of the films were analysed by SEM (refer to section 2.3.2. for SEM samples preparation).

The amount of HA experimentally incorporated into the scaffolds was evaluated using thermogravimetric analyses (TGA 7, Perkin Elmer, USA). Samples were heated from $50^{\circ} \mathrm{C}$ up to $600^{\circ} \mathrm{C}$ at a constant temperature ramp of $10^{\circ} \mathrm{C} / \mathrm{min}$. The final residual masses correspond to the quantities of HA in the scaffolds ( $n=3$ per group).

Additionally, the compressive elastic moduli of the fabricated scaffolds were determined (Z020 tensile and compression tester, Zwick Roell Group, Germany) by recording the stress $(\mathrm{MPa})$ during the mechanical compression of the material between 0 and $10 \%$ strain at a rate of $1 \mathrm{~mm} / \mathrm{min}(28.5 \%$ per $\mathrm{min}$ ) up to $65 \%$. The compressive modulus was calculated between 0 and $10 \%$ strain ( $n=5$ per group).

\subsection{In vitro investigation}

\subsubsection{Primary human BMSCs isolation and cells expansion}

Human bone marrow mesenchymal stem cells (hBMSCs) were isolated from vertebral body bone marrow aspirates (obtained from two donors undergoing spinal fusion with informed consent obtained and full ethical approval (KEK Bern 126/03)). hBMSCs were isolated by means of density centrifugation with the use of Histopaque-1077 (SigmaAldrich). A gated cell count above $8 \mu \mathrm{m}$ (to gate out red blood cells) was performed with the use of a cell Scepter 2.0 Automated Cell Counter (Millipore). Cells were expanded in monolayer at $37^{\circ} \mathrm{C}$ and $5 \% \mathrm{CO}_{2}$ and received media changes 3 times per week, until passage 2 to 3 . The hBMSCs of two donors were expanded individually and used separately for the following experiments.

\subsubsection{Cytocompatibility study}

Following the sterilization, the films were incubated overnight in serum-supplemented media prior to cell seeding. hBMSCs were seeded at 20000 cells $/ \mathrm{cm}^{2}$ and incubated in complete osteogenic medium (OM) based on basic low glucose DMEM (GIBCO) supplemented with $10 \%$ serum (SeraPlus), $50 \mu \mathrm{g} / \mathrm{mL}$ ascorbic acid, $10 \mathrm{nM}$ dexamethasone and 5mM glycerol-2-phosphate (all from Sigma-Aldrich). Tissue Culture Polystyrene (TCPS) and Thermanox coverslips (ThermoFisher, New-York, USA) were used as control surfaces.

Influence of the films composition on cellular adhesion was estimated $24 \mathrm{hrs}$ post-seeding by microscopic observation using SEM and by cells number determination using DNA quantification. SEM analyses required the fixation of the samples overnight in buffered paraformaldehyde at $4 \%$, the dehydration with gradual concentration of ethanol up to $100 \%$ followed by immersion in hexamethyldisilazane (Sigma-Aldrich). After complete drying, the samples were sputter-coated with Pd-Au and investigated using a Hitachi S4700 FESEM instrument. The spreading area of the adherent cells was measured using Image ${ }^{\circledR}$ software, from at least ten different cells per surface and per donor. 
DNA quantification required first the incubation of the cell-seeded films in $200 \mu \mathrm{L}$ of Triton $\mathrm{X}-100$ buffer at $0.1 \%$ in $10 \mathrm{mM}$ of Tris- $\mathrm{HCl}, \mathrm{pH}=7.4$ (all from Sigma-Aldrich). Following 3 hrs of incubation, the supernatant was collected and frozen at $-80^{\circ} \mathrm{C}$ until quantification. After thawing, the amount of DNA in samples was estimated using fluorescent CyQuant ${ }^{\circledR}$ GR Dye assay, according to the supplier's recommendation (Invitrogen), ( $n=3$ per group).

The cytocompatibility of the different photo-crosslinked films on hBMSCs was evaluated using CellTiter Blue assay (Promega, Dübendorf, Switzerland) at 1, 7, 14, 21 and 28 days ( $n=6$ per group), following the supplier's recommendation. For both assays, the resulting fluorescence intensity was read with a multi-plate reader (Viktor ${ }^{3}, 1420$ Multilabel Count, Perkin-Elmer).

\subsection{Statistical analyses}

Statistical analysis of data was performed using Prism software (GraphPad Software, La Jolla, CA, USA). We assumed normal distribution of data. One way ANOVA with Tukey's multiple comparison test was applied to test for significant differences between experimental groups. Data presented are means \pm standard deviation unless stated otherwise.

\section{Results and discussion}

In the field of bioactive scaffolds dedicated to bone tissue engineering, mixing bioactive ceramic-based compounds with bioresorbable polymeric matrices as temporary supportive biomaterials for tissue ingrowth is often considered[16]. Several SLA-fabricated composite structures have already been applied for bone application. However, all the reported biomaterials were based on polyesters macromeres with reactive acrylate/methacrylate (such as PLA, PLGA, PPF...). It is important to note that macromer precursor, reactive moieties or even degradation products released from these scaffolds can cause cytotoxicity and inflammatory reaction. This can result in exacerbated fibrotic reaction and bone resorption[17], which could at the end threaten the fate of the implanted tissue engineered scaffold[4].

The originality of this work is to introduce SLA-fabricated PTMC/HA composite scaffold in the field of bone tissue engineering. Using PTMC as porous matrix could potentially bring advantages compared to polyesters, as no acid by-product is release upon PTMC degradation[18].

\subsection{Resin formulations and influence of HA on PTMC film properties}

PTMC resin formulations with 0, 20 and 40 wt.\% of HA were prepared and given the following abbreviations, PTMC, PTMC 20 and PTMC 40 respectively (table 1). PTMC resins with higher load of HA were also formulated, but they were non-homogeneous, and, in consequence, scaffold SLA-manufacturing using those resins was not possible. Lower concentration of HA was not explored as a high amount of calcium phosphate was deemed necessary to achieve significant biological activity[7]. 
In order to overcome the problem of increased light absorption due to the presence of HA nanoparticles dispersed into the liquid phase, the amount of dye (i.e. Orasol Orange G dye) relative to the concentration of PTMC-MA decreased with increasing amounts of HA, while the concentration of photo-initiator was kept constant at $5 \mathrm{wt} \%$ (table 1). This was first adjusted by assessing the crosslinking and full hardening of thin films of constant thickness and then transposed to 3D macro-porous scaffolds.

Following films fabrication and extraction of the residual solvent, the different surfaces were characterized in terms of hydrophilicity and topography. The addition of HA into the PTMC resulted in a slight, but significant, augmentation of the surface wettability, from 63 $\pm 2^{\circ}$ to $60^{\circ} \pm 1$ for PTMC compared to PTMC 40 (figure 1a). The SEM analysis revealed that the surface of PTMC film was completely smooth, whereas PTMC 20, and more importantly, PTMC 40 films exhibited micro-rough surfaces (figure 1b). HA particles or aggregates of a dimension of approximately $1 \mu \mathrm{m}$ were exposed on the surfaces of the films, with a homogenous repartition.

Table 1. Presentation of the resin formulations used to fabricate films and porous scaffolds by UV crosslinking technique.

\begin{tabular}{lccc}
\hline & \multicolumn{3}{c}{ Resin } \\
\cline { 2 - 4 } Component & PTMC & PTMC 20 & PTMC 40 \\
\hline PTMC-MA (wt.\%) & 60 & 36.4 & 25.1 \\
HA (wt.\%) & 0 & 9.1 & 16.7 \\
Propylene carbonate (wt.\%) & 40.0 & 54.5 & 58.2 \\
Lucirin® TPO-L photo-initiator (wt.\%*) & 5 & 5 & 5 \\
Orasol Orange G dye (wt.\%*) & 0.15 & 0.10 & 0.08 \\
Intended amount of HA in scaffold (wt.\%) & 0 & 20 & 40 \\
\hline
\end{tabular}

*Relative to PTMC-MA.

\subsection{Fabrication of macro-porous PTMC/HA scaffolds using SLA}

Subsequently to the fabrication of films, we evaluated the suitability of the described formulations to be used as photo-crosslinkable resin to fabricate scaffold by stereolithography technique. Three dimensional scaffolds of a dimension of $6 \times 3.5 \mathrm{~mm}$ (diameter $\mathrm{x}$ height) with gyroid porosity were successfully fabricated using a UV stereolithography system (figure 2). Macroscopically, the different scaffolds displayed a high degree of resemblance and shape fidelity in terms of dimension and pore structure independently of the resins' nature (figure $2 \mathrm{a}$ ). 


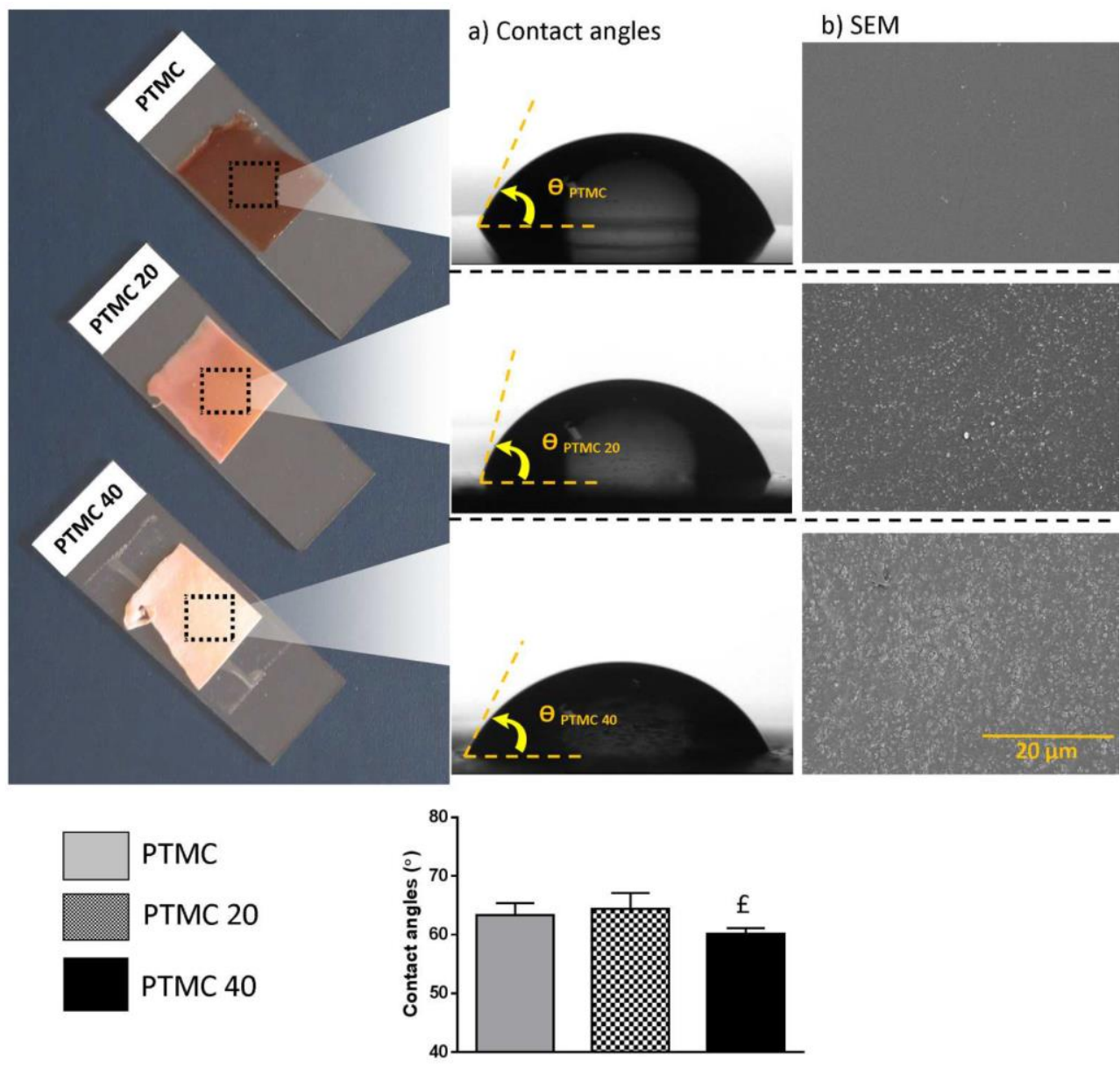

Figure 1. Characterization of the films obtained by photo-crosslinking. Influence of HA loaded in PTMC films on material wettability (measured using direct contact angle (a) and surface roughness investigated using SEM analysis (b)).

The examination of the scaffolds surface using higher magnification correlated with the observations resulting from the SEM analysis of films. Rougher surfaces and agglomerated particles of HA were visible on scaffolds manufactured with PTMC/HA resins in comparison to the one manufactured with PTMC resin (figure $2 b$ ). This has been previously described by Ronca et al. for SLA composite scaffolds using PDLLA matrix[8]. In the mentioned study, the formation of a HA layer covering the surface of SLA-fabricated PDLLA scaffold was observed and attributed to a phase separation between the hydrophilic HA and hydrophobic polymer occurring during the photo-crosslinking process. The presence of HA particles exposed at the surface of the SLA scaffolds could potentially elicit a positive effect on bone healing and material osseointegration. Indeed, coating orthopaedic prostheses with ceramic particles has been widely employed to enhance the 
bioactivity of material implants[19]. However, further in vitro and in vivo experiments will be required to confirm this hypothesis

\section{PTMC}
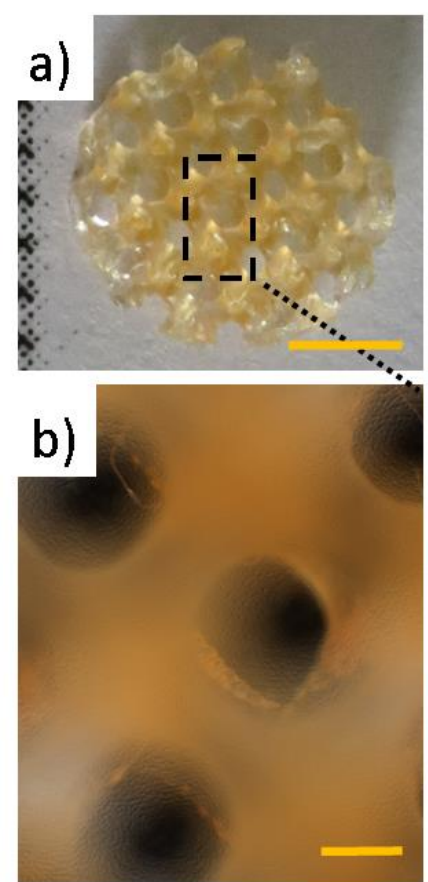

PTMC 20
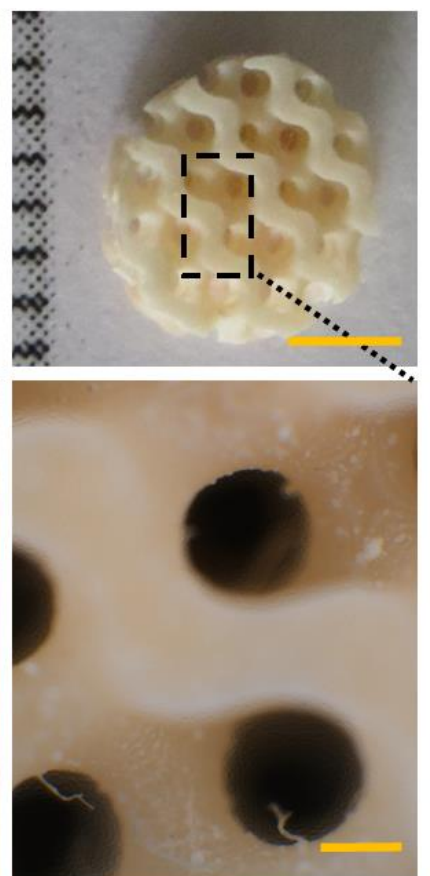

PTMC 40

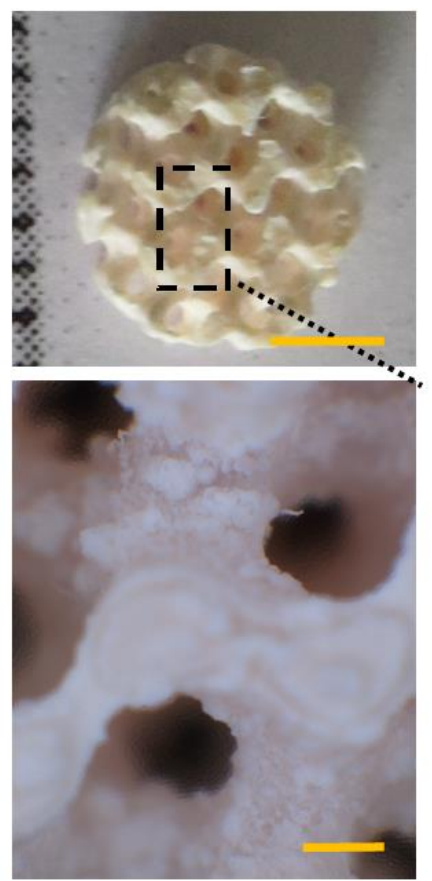

Figure 2. Fabrication of macro-porous scaffolds using SLA. Macroscopic observation of PTMC and PTMC/HA scaffolds (a) with high magnification of the surface of the scaffolds $(b)$.

Thermogravimetric analysis was conducted in order to determine the absolute amount of HA incorporated in the different scaffolds. By recording the residual masses, we observed that PTMC based scaffolds with theoretical HA amount of 0, 20 and $40 \%$ wt. incorporated (table 1) contained experimentally $0.5,23.8$ and $46.5 \%$ wt. of inorganic matters (figure $3 \mathrm{a}$ and $b$ ). The release of un-crosslinked oligomers of PTMC during the extraction process after stereolithography is likely the cause for the slight discrepancies between the experimental and theoretical values.

Subsequently, mechanical compression test was performed on the different groups of scaffold. Data presented figure $3 \mathrm{c}$ and d revealed that incorporation of HA into the PTMC increased the compressive elastic moduli (stiffness of the scaffolds) by up to a factor 9 . From an initial modulus of $0.1 \mathrm{MPa}$ for PTMC scaffolds, the incorporation of $40 \%$ of HA in PTMC resulted in scaffolds with elastic moduli of 0.9 MPa. This increase was mainly due to a reinforcing effect of the dispersed particles in the PTMC matrix. Changes in SLA scaffold porosity and micro-structure could also have influenced the compressive moduli, 
although probably to a less extend because of the relatively good shape fidelity between the different groups.

Finally, the flexibility and the trabecular-like structure of the crosslinked PTMC was conserved in all the composite scaffolds. This advantageous property could allow to dynamically load the scaffolds for in vitro cell culture in bioreactor or to use such scaffolds as press-fit porous filler for bone defects[20], as alternative to CaP-based granules.

a)

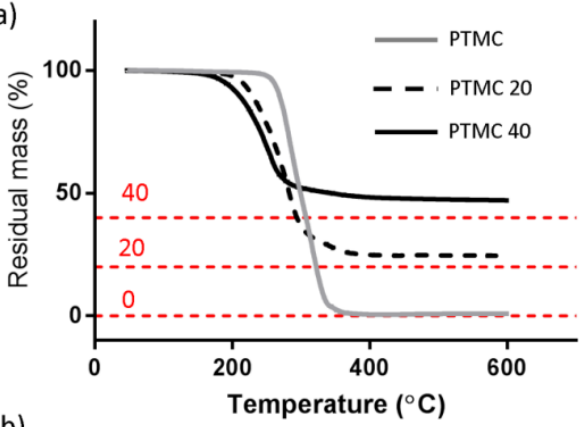

b)

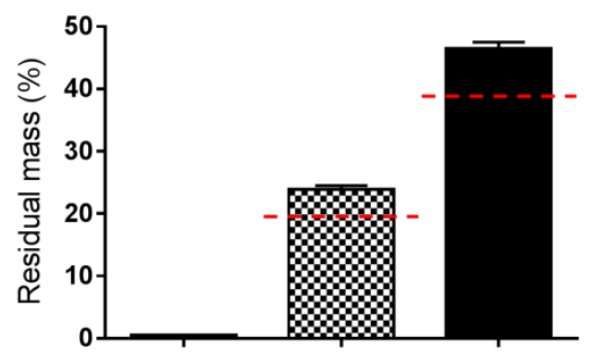

c)

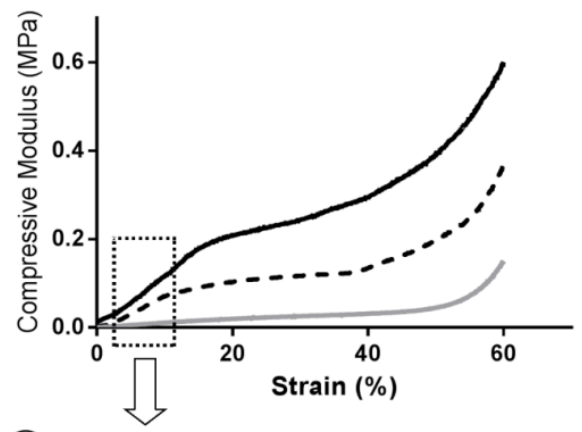

d)

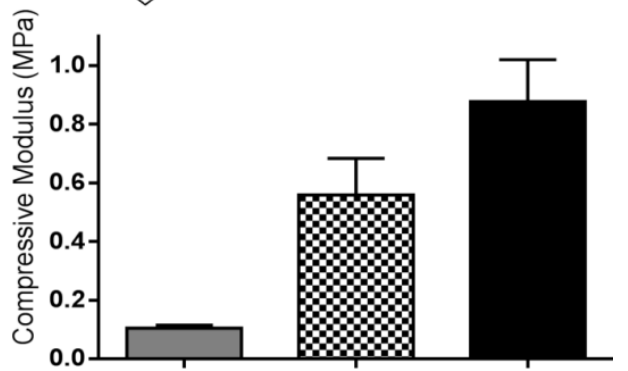

PTMC 20
PTMC 40

Figure 3. Characterization of the SLA-fabricated scaffolds. Quantification of the amount of HA incorporated into the scaffolds using thermal analysis (TGA, estimated by the residual mass (\%), with the red dashed lines representing the targeted values ( $a$ and $b)$ ). Investigation of the influence of HA particles incorporated into PTMC scaffolds on the mechanical properties (c) and on the compressive elastic moduli of the scaffolds (d).

\subsection{Cytocompatibility of PTMC/HA biomaterials}

hBMSCs were seeded onto photo-crosslinked PTMC, PTMC 20 and PTMC 40 surfaces and attachment, cells morphology and proliferation assessed against optimised cell culture surfaces, tissue culture polystyrene (TCPS) and Thermanox ${ }^{\mathrm{TM}}$ (figure 4). At 24 hours, the DNA content measured on the surface of PTMC, PTMC 20 and PTMC 40 was similar to TCPS (figure $4 \mathrm{a}$ ). The presence of HA in PTMC did not influence the number of adherent cells at 24 hours. No major difference could be detected between the different produced surfaces and Thermanox ${ }^{\mathrm{TM}}$ regarding the morphology of the adhering hBMSCs, although 
hBMSCs seeded on PTMC 40 exhibited a trend toward smaller surface area (figures $4 \mathrm{~b}$ and c). All the cells exhibited elongated filopodia and flattened cytoplasm.

a)

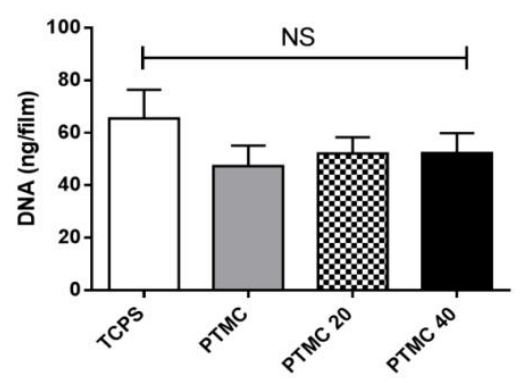

c)

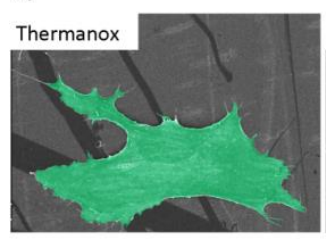

b)

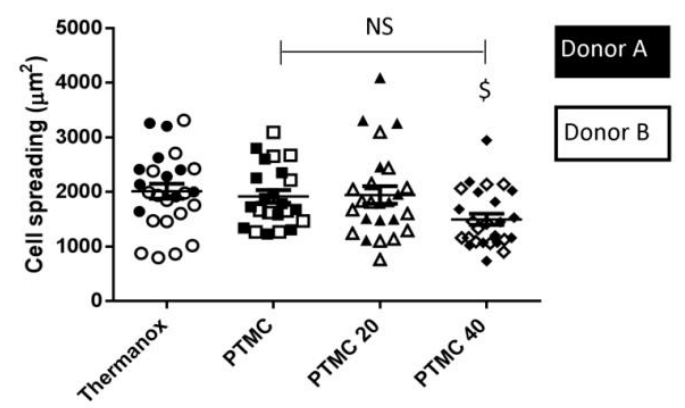

Figure 4. In vitro adhesion study. Ability of hBMSCs to adhere on the surface of the biomaterials, studied 24 hrs post-seeding by quantification of the amount of DNA per group (a), by measuring cell spreading area using image analysis ( $b$, \$ indicates significance compared to control surface TCPS for donor A (no difference for donor $B$ was noted), mean +/- standard error of the mean) and using SEM (c, illustration donor A, with cells highlighted by artificial green colouring using Photoshop ${ }^{\circledast}$ ).

Over a period of 28 days post-seeding, all the tested surfaces were prone to cell colonization (figure 5a). A dense monolayer of cells could be observed, independently to the nature of the surfaces. Additionally, a metabolic activity assay performed weekly revealed that the proliferation kinetic was similar for positive control (TCPS) and for the surfaces of PTMC without or with HA for both donors (figure $5 \mathrm{~b}$ and c). Within 14 to 21 days, the cells became confluence and covered the entire surface of the different films.

This study confirms that the chemical nature of the photo-crosslinked composite PTMC/HA is cytocompatible. Secondly, it demonstrates that the cleansing process performed after stereolithography is efficient to clear toxic level of residual solvent and photo-reagent products present initially in the resin formulations. Interestingly, adding HA into the PTMC did not influence significantly the cytocompatibility and behaviour of hBMSCs, although the surface topography was significantly modified.

Further in vitro experiments are required to investigate the impact of HA load into photocrosslink PTMC resin on hBMSCs osteogenic differentiation. 
a)
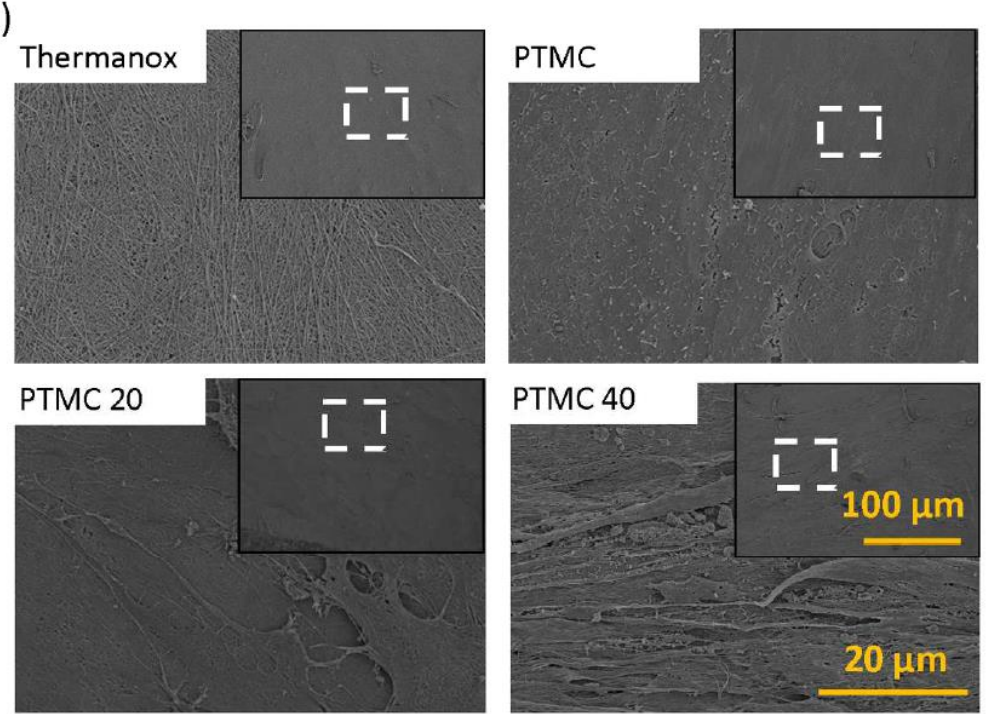

b)
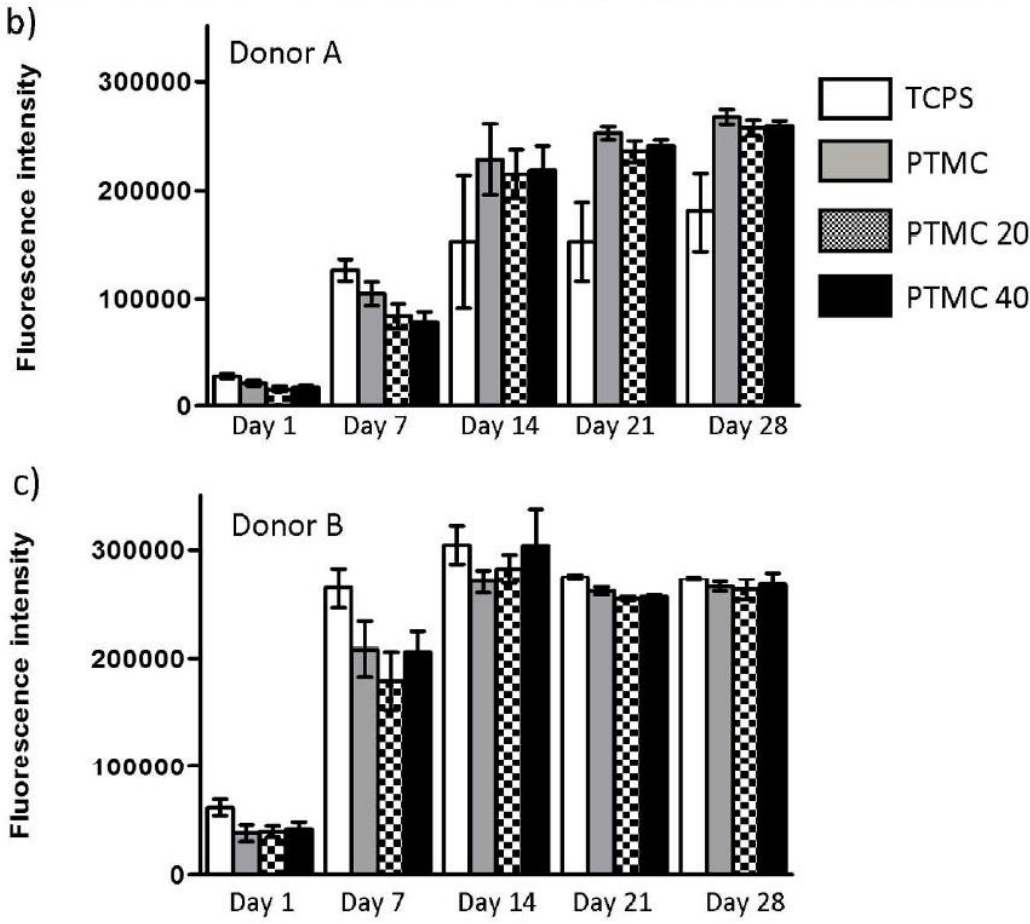

Figure 5. In vitro cytocompatibility study. Cell colonization of the different biomaterial surfaces, illustrated by SEM observation (a, following 28 days of incubation, donor A) and was quantified using metabolic assay for donors $A$ and $B$ ( $b$ and $c$ respectively). 


\section{Conclusions}

In this feasibility study, we prepared various composite resin formulations based on poly(trimethylene carbonate) PTMC and nano-hydroxyapatite (HA) in order to create macro-porous scaffolds using stereolithography. Up to $40 \%$ of HA compared to PTMC (in weight \%) could be loaded in films and in macro-porous scaffolds. This resulted in an increased hydrophilicity, surface roughness and mechanical property, compared to PTMC only. Subsequently, we demonstrated in vitro that SLA-fabricated PTMC/HA exhibited satisfying cytocompatibility on hBMSCs. To summarize, this work is of substantial importance in bone engineering application, as it opens the field of PTMC-based scaffolds to stereolithography manufacturing technology.

\section{Acknowledgements}

Authors would like to acknowledge NSFC-DG-RTD Joint Scheme (Project No. 51361130034) and the European Union's 7th Framework Program under grant agreement $\mathrm{n}^{\circ}$ NMP3-SL-2013-604517, Project RAPIDOS. Additionally, we are grateful to Eberli U., Dr. Stadelmann V., Dr. Nehrbass, D. and Dr. Sprecher C. for their technical assistance.

\section{References}

1. Ventola, C.L., Medical Applications for 3D Printing: Current and Projected Uses. P T, 2014. 39(10): p. 704-711.

2. Bose, S., M. Roy, and A. Bandyopadhyay, Recent advances in bone tissue engineering scaffolds. Trends in Biotechnology, 2012. 30(10): p. 546-554.

3. Schuller-Ravoo, S., E. Zant, J. Feijen, and D.W. Grijpma, Preparation of a designed poly(trimethylene carbonate) microvascular network by stereolithography. Advanced Healthcare Materials, 2014. 3(12): p. 2004-2011.

4. Do, A.V., B. Khorsand, S.M. Geary, and A.K. Salem, 3D Printing of Scaffolds for Tissue Regeneration Applications. Advanced Healthcare Materials, 2015. 4(12): p. 1742-1762.

5. Goodman, S.B., Z. Yao, M. Keeney, and F. Yang, The future of biologic coatings for orthopaedic implants. Biomaterials, 2013. 34(13): p. 3174-3183.

6. Lee, K.W., S. Wang, B.C. Fox, E.L. Ritman, M.J. Yaszemski, and L. Lu, Poly(propylene fumarate) bone tissue engineering scaffold fabrication using stereolithography: effects of resin formulations and laser parameters. Biomacromolecules, 2007. 8(4): p. 1077-1084.

7. Elomaa, L., A. Kokkari, T. Närhi, and J.V. Seppälä, Porous 3D modeled scaffolds of bioactive glass and photocrosslinkable poly(e-caprolactone) by stereolithography. Composites Science and Technology, 2013. 74: p. 99-106.

8. Ronca, A., L. Ambrosio, and D.W. Grijpma, Design of porous three-dimensional PDLLA/nano-hap composite scaffolds using stereolithography. J Appl Biomater Funct Mater, 2012. 10(3): p. 249-258. 
9. Ronca, A., L. Ambrosio, and D.W. Grijpma, Preparation of designed poly(D,Llactide)/nanosized hydroxyapatite composite structures by stereolithography. Acta Biomater, 2013. 9(4): p. 5989-5996.

10. Song, Y., M.M.J. Kamphuis, Z. Zhang, L.M.T. Sterk, I. Vermes, A.A. Poot, J. Feijen, and D.W. Grijpma, Flexible and elastic porous poly(trimethylene carbonate) structures for use in vascular tissue engineering. Acta Biomater, 2010. 6(4): p. 1269-1277.

11. van Leeuwen, A.C., J.J.R. Huddleston Slater, P.F.M. Gielkens, J.R. de Jong, D.W. Grijpma, and R.R.M. Bos, Guided bone regeneration in rat mandibular defects using resorbable poly(trimethylene carbonate) barrier membranes. Acta Biomater, 2012. 8(4): p. 1422-1429.

12. van Leeuwen, A.C., R.R. Bos, and D.W. Grijpma, Composite materials based on poly(trimethylene carbonate) and beta-tricalcium phosphate for orbital floor and wall reconstruction. Journal of Biomedical Materials Research Part B - Applied Biomaterials, 2012. 100(6): p. 1610-1620.

13. van Leeuwen, A.C., H. Yuan, G. Passanisi, J.W. van der Meer, J.D. de Bruijn, T.G. van Kooten, D.W. Grijpma, and R.R. Bos, Poly(trimethylene carbonate) and biphasic calcium phosphate composites for orbital floor reconstruction: a feasibility study in sheep. European Cells \& Materials, 2014. 27: p. 81-96 ; discussion 96-7.

14. Chapter 3 of this thesis; Geven, M.A., D. Barbieri, H. Yuan, J.D. de Bruijn, and D.W. Grijpma, Preparation and mechanical properties of photo-crosslinked poly(trimethylene carbonate) and nano-hydroxyapatite composites. Clinical Hemorheology and Microcirculation, 2015. 60(1): p. 3-11.

15. Chapter 4 of this thesis; Geven, M.A., V. Varjas, L. Kamer, X. Wang, J. Peng, D. Eglin, and D.W. Grijpma, Fabrication of patient specific composite orbital floor implants by stereolithography. Polymers for Advanced Technologies, 2015. 26(12): p. 1433-1438.

16. Bose, S., S. Vahabzadeh, and A. Bandyopadhyay, Bone tissue engineering using $3 D$ printing. Materials Today, 2013. 16(12): p. 496-504.

17. Taylor, M.S., A.U. Daniels, K.P. Andriano, and J. Heller, Six bioabsorbable polymers: in vitro acute toxicity of accumulated degradation products. Journal of Applied Biomaterials, 1994. 5(2): p. 151-157.

18. Bat, E., T.G. van Kooten, J. Feijen, and D.W. Grijpma, Resorbable elastomeric networks prepared by photocrosslinking of high-molecular-weight poly(trimethylene carbonate) with photoinitiators and poly(trimethylene carbonate) macromers as crosslinking aids. Acta Biomater, 2011. 7(5): p. 19391948.

19. Yang, Y., K.-H. Kim, and J.L. Ong, A review on calcium phosphate coatings produced using a sputtering process--an alternative to plasma spraying. Biomaterials, 2005. 26(3): p. 327-337. 


\section{Chapter 6}

20. Peroglio, M., L. Gremillard, D. Eglin, P. Lezuo, M. Alini, and J. Chevalier, Evaluation of a new press-fit in situ setting composite porous scaffold for cancellous bone repair: towards a "surgeon-friendly" bone filler? Acta Biomater, 2010. 6(9): p. 3808-3812. 


\title{
Chapter 7 - Surface-enrichment with hydroxyapatite nanoparticles in stereolithography-fabricated composite polymer scaffolds promotes bone repair
}

Olivier Guillaume ${ }^{\mathrm{a}}$, Mike A. Geven ${ }^{\mathrm{a}}$, Christoph M. Sprecher ${ }^{\mathrm{a}}$, Vincent A. Stadelmann ${ }^{\mathrm{a}}$, Dirk W. Grijpma ${ }^{b}$, Ting-Ting Tang ${ }^{c}$, Ling Qin ${ }^{d}$, Yuxiao Lai ${ }^{d}$, Mauro Alini ${ }^{\mathrm{a}}$, Joost D. de Bruijn $^{\mathrm{e}}$, Huipin Yuan ${ }^{\mathrm{e}}$, Robert G. Richards ${ }^{\mathrm{a}}$ and David Eglin ${ }^{\mathrm{a}}$

${ }^{a}$ AO Research Institute, Davos, Switzerland

${ }^{\mathrm{b}}$ Department of Biomaterials Science and Technology, University of Twente, Enschede, The Netherlands

${ }^{c}$ Shanghai Key Laboratory of Orthopaedic Implants, Department of Orthopaedic Surgery, Shanghai Ninth People's Hospital, Shanghai Jiao Tong University School of Medicine, Shanghai, People's Republic of China

${ }^{\mathrm{d}}$ Translational Medicine Research and Development Centre, Institute of Biomedical and Health Engineering, Shenzhen Institute of Advanced Technology, The Chinese Academy of Sciences, Shenzhen, People's Republic of China

${ }^{\mathrm{e}}$ Kuros BioSciences BV, Bilthoven, The Netherlands

\begin{abstract}
:
Fabrication of composite scaffolds using stereolithography (SLA) for bone tissue engineering has shown great promises. However, in order to trigger effective bone formation and implant integration, exogenous growth factors are commonly combined to scaffold materials. In this study, we fabricated biodegradable composite scaffolds using SLA and endowed them with osteopromotive properties in the absence of biologics. First we prepared photo-crosslinkable poly(trimethylene carbonate) (PTMC) resins containing 20 and 40 wt. \% of hydroxyapatite (HA) nanoparticles and fabricated scaffolds with controlled macro-architecture. Then, we conducted experiments to investigate how the incorporation of HA in photo-crosslinked PTMC matrices improved human bone marrow stem cells osteogenic differentiation in vitro and kinetic of bone healing in vivo. We observed that bone regeneration was significantly improved using composite scaffolds containing as low as $20 \mathrm{wt}$. \% of HA, along with difference in terms of osteogenesis and degree of implant osseointegration. Further investigations revealed that SLA process was responsible for the formation of a rich microscale layer of HA corralling scaffolds. To summarize, this work is of substantial importance as it shows how the fabrication of hierarchical biomaterials via surface-enrichment of functional HA nanoparticles in composite polymer stereolithographic structures could impact in vitro and in vivo osteogenesis.
\end{abstract}

Published:

Acta Biomaterialia, 2017, 54, 386-398 


\section{Introduction}

Bone is the most frequently transplanted tissue after blood, and the need for effective and patient specific bone graft substitute materials, supplanting autografts and allografts, remains enormous[1]. Additive manufacturing (AM) technologies have emerged as promising alternatives to classical fabrication processes for bone repair materials based on patient's specific information[2]. Among AM techniques, stereolithography (SLA) was one of the first introduced, three decades ago by Chuck Hull, as a system "able to generate $3 D$ objects from a fluid medium by step-wise and successive alteration through specific stimulation"[3] and is still nowadays nourishing major innovations. Indeed, SLA has shown great interest in the field of tissue repair due to the high geometric resolution achieved[4], which remains extremely challenging to approach with other AM technologies, especially when considering the layer-by-layer fabrication of calcium phosphate $(\mathrm{CaP})$-containing biomaterials.

SLA technique brings tremendous advantages in terms of control of the internal macro/micro-architecture, of the degree of porosity and organization of the pores and, more importantly, in terms of freedom regarding the customized composition, design and shape of the fabricated biomaterial implants[2]. More specifically for bone repair purposes, an overview of the polymer/CaP composite structures fabricated so far using SLA indicated that, mostly biodegradable polyesters resins with a dispersion of micro- and nanoparticles of $\mathrm{CaP}$ ceramic (as high as $60 \mathrm{wt} . \%$ ) have been proposed (supplementary table 1). From the presented list, it is clear that the most widely employed biodegradable polymers are represented by the family of the polyesters. Nevertheless, a new biodegradable and biocompatible polycarbonate polymer (i.e. poly(trimethylene carbonate), PTMC) has just been introduced to this specific biomedical field of application[5, 6]. Exploratory investigations revealed that PTMC-based biomaterials exhibit unique and versatile features, regarding numerous parameters (such as the kinetic and the mechanism of resorption, the mechanical properties, the chemical functionalizations, etc.) compared to common alternatives such as biodegradable aliphatic polyesters (including poly(lactic acid) (PLA), poly(glycolic acid) (PGA) or poly( $\varepsilon$-caprolactone) (PCL))[7].

As instance, for all these synthetic biodegradable polyesters, the degradation mechanism is relatively similar, driven by hydrolysis of the ester linkages, releasing acid by-products[8], which can cause inflammatory reaction, upon local acidic oligomers accumulation, and exacerbate fibrotic reaction and bone resorption[9, 10]. In opposition to the hydrolytic degradation of the above-mentioned polyesters, PTMC degradation is in majority driven by enzyme and oxidative species via a surface erosion mechanism[11]. Additionally, the structural mechanical integrity of the PTMC tends to be better maintained overtime attributed to this singular erosion mechanism[7, 12, 13]. Fabrication of PTMC scaffolds using SLA has already been widely investigated, but without focusing on bone tissue repair yet. We hypothesized that SLA-fabricated composite PTMC scaffolds could exhibit advantageous features compared to the available biomaterials presented (supplementary table 1). 
Noteworthy, none of the reported composite microstructures fabricated by stereolithography promoted significant bone formation in vivo in the absence of additive factors, such as BMP-2[14, 15]. One of the reasons is that the main composite scaffolds are constituted of inorganic $\mathrm{CaP}$ particles physically entrapped in photo-crosslinked polymeric matrices. We postulated that the restricted exposition of $\mathrm{CaP}$ to the external environment combined with inadequate degradation kinetic of composite SLA-scaffolds, hinder effective bone healing capacity[16]. Controlling spatial arrangement of CaP particles inside $3 \mathrm{D}$ printed scaffolds could alleviate this limitation. Interestingly, organized nano/microscale assemblies of loaded nanoparticles in polymer composites have been investigated in the field of electronic, optic, photovoltaic and self-healing materials[17, 18]. Such nano/microscale particle arrangements are actually dictated by the size and radius, the shape, the volume fraction and importantly, the surface interaction between the nanoparticles and the polymer matrix[19]. As a result, disparate nanoparticles in a polymer mixture can self-organize at the interface of immiscible solvents and polymer blends that leads to hierarchically structured composites[17]. Controlling this spatial segregation has been achieved in extrusion and solvent casting processes[20], but interestingly not exploited yet in 3D printing and stereolithography manufacturing.

In this study, using in vitro and in vivo experiments, we first deciphered how the developed SLA-PTMC/HA composite structures commanded osteogenesis and drove the bone healing cascade. Topographical analyses performed on the scaffolds revealed that stereolithography processing resulted in a surface-enrichment with agglomerated HA nanoparticles at the surface interface of the disparate PTMC/HA scaffold. This investigation is of great importance as it paves the way for further optimizations regarding the modulation of nanoparticles spatial arrangement in 3D-printed structures.

\section{Experimental section}

\subsection{Preparation of photo-crosslinked PTMC/HA composite macro-porous scaffolds}

Three-armed poly(trimethylene carbonate) (PTMC) was synthesized by ring-opening polymerization of trimethylene carbonate with trimethylolpropane using $\mathrm{Sn}(\mathrm{Oct})_{2}$ as catalyst [5, 6]. A PTMC macromer with methacrylate end-groups (PTMC-MA) was subsequently prepared by reacting PTMC hydroxyl groups with methacrylic anhydride[6]. Different resin formulations were prepared with a varying content of nano-hydroxyapatite (HA). Kuros BioSciences BV (Bilthoven, The Netherlands) kindly provided HA in the form of a powder with aggregates $(\varnothing=15 \mu \mathrm{m})$ of needle-like HA crystals of 200 to $400 \mathrm{~nm}$ long and 20 to $50 \mathrm{~nm}$ wide. Those nano-HA particles were prepared using a wetprecipitation technique[21]. A stoichiometric $\mathrm{Ca} / \mathrm{P}$ ratio of 1.67 was selected to produce the needle-like HA particles, similar to the mineral composition and shape of HA in human bone.

First, PTMC-MA and HA were dispersed in chloroform and precipitated in cold methanol. The resulting dried PTMC-MA/HA powder was mixed with propylene carbonate diluent 
(Merck Millipore), Lucirin ${ }^{\circledR}$ TPO-L photo-initiator (BASF) and Orasol Orange G dye (from Ciba Speciality Chemicals).

From the resins, scaffolds (6 x $3.5 \mathrm{~mm}$, diameter $\mathrm{x}$ height) with gyroid porosity were fabricated using an Envisiontec Perfactory ${ }^{3{ }^{\circledR}}$ SXGA $^{+}$Standard UV stereolithography system. A light intensity of $180 \mathrm{~mW} / \mathrm{dm}^{2}$ was used with an irradiation time of 9 seconds per layer and the platform was elevated by $50 \mu \mathrm{m}$ between each layer. The internal architecture parameters of the manufactured scaffolds were designed suitable to allow for vascularisation and bone ingrowth/formation[22], with minimal pore size of $600 \mu \mathrm{m}$, porosity of $70 \%$ and interconnectivity $100 \%$. Following materials fabrication, propylene carbonate diluent was removed by extraction in propylene carbonate/ethanol $(1 / 1 \mathrm{v} / \mathrm{v})$, gradually exchanged to $100 \%$ ethanol, the scaffolds were then dried and sterilized using cold ethylene oxide gas procedure.

For the experiments performed on 2D surfaces, films were prepared by solvent casting of the resins on glass plates and subsequent photo-crosslinking in a UV-crosslinking box at $365 \mathrm{~nm}$ and $1100 \mathrm{~mW} / \mathrm{dm}^{2}$ for 5 min under $\mathrm{N}_{2}$. Following fabrication, films' purification and sterilization were performed the same way as previously described for porous scaffolds.

\subsection{Materials characterization}

The architecture of the scaffolds was analysed by Scanning Electron Microscopy (SEM, refer to section 2.3.1.) and micro-computed tomography (microCT). Briefly, the volume fraction of porosity, the trabecular thickness and trabecular space were measured (corresponding to the strut and pore diameters respectively) using bone trabecular morphometry script $(n=3$, with a microCT 40; $30 \mu$ m voxel size, SCANCO Medical AG, Bruettisellen, Switzerland). Additionally, HA particles repartition in the trabecular structure of the porous scaffolds was observed using a high-resolution microCT scanner (microCT 100; $3 \mu \mathrm{m}$ voxel size).

Calcium ions released from the scaffolds (weighing $45+/-2 \mathrm{mg}$ ) immersed in phosphate saline buffer (PBS) at $37^{\circ} \mathrm{C}(1 \mathrm{~mL})$ was assessed. At day 1, 3, 4, 7, 12, 19 and 28, the supernatant was removed and replaced by fresh PBS. The quantification of calcium in the supernatant was performed via the formation of chromogenic reagent by complexation of $\mathrm{Ca}^{2+}$ with o-cresolphthalein (Calcium Colorimetric Assay Kit, Sigma-Aldrich) measured at $560 \mathrm{~nm}$ (n=3 per group).

Protein adsorption was investigated by incubating scaffolds in PBS containing $10 \%$ of Serum (SeraPlus, Milan Analytica, Basel, Switzerland), at $37{ }^{\circ} \mathrm{C}$ for 5 days, using Quick Start $^{\mathrm{TM}}$ Bradford protein kit (Bio-Rad Laboratories, Cressier, Switzerland), according to the manufacturer's protocol using bovine serum albumin (BSA) as standard ( $n=3$ per group).

Swelling/shrinkage was estimated by incubating scaffolds for 28 days in sterile PBS and conducting microtomography scans (using microCT 40) of the same scaffold before and after drying (48 hrs at $40{ }^{\circ} \mathrm{C}$ under vacuum at 200 mbar). Swelling quantification was 
expressed in percentage of the trabecular thickness (Tr. Th.) of the scaffold ( $n=3$ per group) and calculated using the equation 1 .

$$
\text { Swelling }(\%)=\operatorname{Tr} . \operatorname{Th} \frac{\text { wet-dry }}{\text { wet }} * 100
$$

Additionally, the wettability of the different surfaces was characterized using the contact angle method on films (Drop Shape Analysis System, DSA10 Krüss, Germany, n=10 per group).

\subsection{In vitro investigation}

Human bone marrow mesenchymal stem cells (hBMSCs) were isolated from vertebral body bone marrow aspirates (obtained from donors undergoing spinal fusion with informed consent and full ethical approval (KEK Bern 126/03)). hBMSCs of two donors were expanded individually[23] and used separately (at passage 3) for the following experiments, but all the values were finally pooled in the presented histograms.

\subsubsection{In vitro investigation of scaffolds colonization and matrix deposition}

SLA-fabricated scaffolds were incubated overnight in complete osteogenic medium (OM) (based on basic low glucose DMEM (GIBCO) supplemented with $10 \%$ serum (SeraPlus), $50 \mu \mathrm{g} / \mathrm{mL}$ ascorbic acid, $10 \mathrm{nM}$ dexamethasone and $5 \mathrm{mM}$ glycerol-2-phosphate (all from Sigma-Aldrich)), before seeding with $150 \mu \mathrm{L}$ of a cell suspension containing $1 \times 10^{6}$ hBMSCs/mL.

After $8 \mathrm{hrs}$, the seeded scaffolds were moved to a new low-adhering 24 well-plate (Nunclon Sphera, ThermoFisher Scientific, Zürich) in order to prevent cells to proliferate on the bottom of the plate, and incubated with OM (1 mL per well, changed three times a week). 24 hrs and 28 days post-seeding, samples were rinsed with PBS and subjected to various analyses.

Cell viability was evaluated with Live/Dead ${ }^{\circledR}$ staining. The samples were incubated with calcein acetoxymethylester and ethidium homodimer, at 10 and $1 \mu \mathrm{g} / \mathrm{mL}$ respectively, washed and imaged using fluorescent confocal microscope (Axiovert 200m microscope equipped with an AxiocamHRc and Axiovision software (Zeiss, Gottingen, Germany)).

SEM analysis was performed after fixation in buffered paraformaldehyde at $4 \%$, followed by gradual dehydration in ethanol and by immersion in hexamethyldisilazane (SigmaAldrich). After drying, the samples were sputter-coated with Au-Pd and investigated by electron microscopy using a Hitachi S4700 FESEM (Hitachi, Japan). Meanwhile, the chemical composition of the scaffolds was determined by energy dispersive $\mathrm{X}$-ray (EDX, Oxford Instruments, UK), following $\mathrm{C}$ coating.

The amount of DNA present in samples was quantified following samples digestion in proteinase $\mathrm{K}$ solution at $0.5 \mathrm{mg} / \mathrm{mL}$ (Roche, Mannheim, Germany) for $16 \mathrm{hrs}$ at $56^{\circ} \mathrm{C}$, and reaction using fluorescent Bisbenzimide Hoechst 33258 at an excitation of $360 \mathrm{~nm}$ and an emission of $465 \mathrm{~nm}$ ( $\mathrm{n}=3$ per group). 
Collagen deposition was evaluated following specimen fixation in methanol $70 \%$, incubation in PBS containing $5 \%$ sucrose (w/v) and immersion in cryo-compound (Tissue freezing Medium from Leica Biosystems, Muttenz, Switzerland) before cryosection (section of $8 \mu \mathrm{m}$, Cryostat-Microtome HM 500 OM from Carl-Zeiss, Zürich, Switzerland ). Safranin-O Fast Green staining (counter-stained with Weigert's Haematoxylin) was realized on cryo-sections ( $n=2$ per group).

\subsection{2. hBMSCs osteogenic differentiation on PTMC/HA}

hBMSCs were seeded on the different material films $(6 \mathrm{~mm} \varnothing)$ at $20 \times 10^{3} \mathrm{cells} / \mathrm{cm}^{2}$ and incubated in complete osteogenic medium (OM) for 4 weeks (in 96 well-plate using $100 \mu \mathrm{L}$ of medium per well, changed three time a week), using Tissue Culture Polystyrene (TCPS) as control. As the films covered firmly the entire bottom surface of the well plate, preventing cells to proliferate on the underlying TCPS, no exchange to new well plate was performed post-seeding as it was reported for 3D scaffolds (section 2.3.1).

For DNA quantification, samples were first incubated in lysis buffer made of Triton X-100 at $0.1 \%$ in $10 \mathrm{mM}$ of Tris- $\mathrm{HCl}, \mathrm{pH}=7.4$ (all from Sigma-Aldrich) and followed by one freezing-thawing cycle. Then, DNA amount was estimated using fluorescent CyQuant ${ }^{\circledR}$ GR Dye assay, according to the supplier's recommendation (Invitrogen), ( $\mathrm{n}=3$ per group). Alkaline phosphatase activity (ALP) from the cell-lysis solution was determined using colorimetric quantification. Briefly, samples along with a set of standard solutions ( $p$ nitrophenol of concentrations from 0 to $1000 \mu \mathrm{M}$ ) were incubated with alkaline buffer solution (2-amino-2-methylpropanol 1.5 M pH=10.3, from Sigma-Aldrich) and then ALP substrate buffer was added (phosphatase substrate dissolved in diethanolamine buffer at 1 $\mathrm{M}$ in $0.5 \mathrm{mM} \mathrm{MgCl}$ adjusted $\mathrm{pH}=9.8$ ). After mixing, and heating (at $37{ }^{\circ} \mathrm{C}$ for exactly 15 min), a solution of $\mathrm{NaOH}$ at $0.1 \mathrm{M}$ was added to each tube in order to stop the reaction. Then, the intensity of $p$-nitrophenol formation was monitored at $405 \mathrm{~nm}$. The total ALP contents were expressed as enzyme activity units in $\mathrm{nmol} / \mathrm{min}$ ( $\mathrm{n}=3$ per group), as a function of total DNA (ng) per well measured using CyQuant ${ }^{\circledR}$ assay.

Presence of mineralization was detected using Alizarin Red Staining assay (ARS, SigmaAldrich), with Thermanox plastic coverslips $\left(\mathrm{NUNC}^{\mathrm{TM}}\right.$ Thermanox $^{\mathrm{TM}}$ Coverslips, from ThermoFisher) used as control films (for macroscopic and SEM observations). The cell monolayer was washed with PBS, fixed with formaldehyde $4 \%$ and further washed with deionised (DI) water. Then, $40 \mathrm{mM}$ ARS solution at $\mathrm{pH}=4.2$ was added to each well for 1 $\mathrm{hr}$ and thoroughly washed with DI water for 5 days. Finally, samples were imaged by light microscopy (Macrofluo ${ }^{\mathrm{TM}}$ from Leica) and a quantification of the ARS was performed by acid extraction thereafter. Briefly, acetic acid (at $10 \%$ ) was added to each well for $30 \mathrm{~min}$, and the loosely attached monolayer of cells was transferred to Eppendorf tubes and heated up to $85^{\circ} \mathrm{C}$ for $10 \mathrm{~min}$, then placed on ice for $5 \mathrm{~min}$. After centrifugation at $20000 \mathrm{~g}$ for 15 min, ammonium hydroxide was added to the supernatant (final $\mathrm{pH}$ of 4.1-4.5) and absorbance was recorded at $405 \mathrm{~nm}$ and compared to ARS standard solutions ranged from 
0 up to $2000 \mu \mathrm{M}$ ( $\mathrm{n}=3$ per group) (ARS intensities in cell-free condition at day 1 and 28 are shown as controls).

Additionally, to analyse the effect of the incorporation of HA into the PTMC matrix on the osteogenic gene expression (collagen type I (h-Col 1) and h-ALP), 3x10 5 hBMSCs were seeded on the different scaffolds and cultivated for 14 days in basal and osteogenic media (BM and OM respectively). BM contained only $\alpha$-MEM supplemented with $10 \%$ of SeraPlus and OM composition was previously described (section 2.3.1). Total RNA was isolated from TRI Reagent (Molecular Research Center, MRC) containing Polyacryl Carrier $^{\mathrm{TM}}$ (at $2 \mu \mathrm{L} / \mathrm{mL}$ ) according to the manufacturer's protocol, and the RNA was controlled qualitatively and quantitatively by spectrophotometry. $1000 \mathrm{ng}$ of RNA was used for cDNA synthesis using TaqMan Reverse Transcription reagents (from Applied Biosystems) and loaded in a Thermocycler (GeneAmp 5700SDS instrument). PCR was performed on a QuantStudio ${ }^{\text {TM }} 6$ Flex Real-Time PCR System using TaqMan universal Master Mix reagents. Fold induction calculated using the $\Delta \mathrm{CT}$ method after normalization with Fast $18 \mathrm{~S}$ as a housekeeping gene and relative to cells grown on PTMC scaffold (without HA) in BM condition ( $n=3$ per condition and per donor).

\subsection{Animal model experiment}

\subsubsection{Operative procedure and surgical implantation}

Calvarial defect on rabbits $(n=8$, specific pathogen free, New Zeeland-White rabbit, female, skeletally mature > 24 weeks, $4.0 \pm 0.3 \mathrm{~kg}$ from Charles River Laboratory) was conducted following the approval of the ethical committee of the canton of Grisons in Switzerland (GR 19A/2015). All procedures were performed in an AAALAC International (Association for Assessment and Accreditation of Laboratory Animal Care) approved facility and according to Swiss animal protection law and regulations. Animals were sedated with 0.2 $\mathrm{mg} / \mathrm{kg}$ Medetomidim (Domitor ${ }^{\circledR}$, Pfizer, Switzerland), $0.2 \mathrm{mg} / \mathrm{kg}$ Midazolam (Dormicum ${ }^{\circledR}$, Roche Pharma AG; Switzerland) and $0.005 \mathrm{mg} / \mathrm{kg}$ Fentanyl (Sintenyl ${ }^{\circledR}$, Sintetica SA, Switzerland) intramuscularly. General anaesthesia was induced with Propofol (Propofol ${ }^{\circledR} 1$ $\%$ Fresenius, Fresenius Kabi AG, Switzerland) intravenously and animals were intubated and maintained at 1.5 to $2 \%$ Isoflurane (Isofluran Baxter ${ }^{\circledR}$, Baxter AG, Switzerland) in oxygen. $4 \mathrm{mg} / \mathrm{kg}$ Carprofene (Rimadyl ${ }^{\circledR}$, Pfizer AG, Switzerland) intravenously was used as pre-emptive analgesia together with $2-3 \mathrm{mg} / \mathrm{kg}$ ropivacaine applied locally.

After incision made from the nasal bone to the occipital crest and reflection of the soft tissue, the parietal bones were exposed. Four evenly distributed $6 \mathrm{~mm}$ diameter defects (non-critical size) were created with a Codman perforator apparatus (commercialized by Depuy-Synthes). The created defects were randomly assigned to 4 different groups, including empty defects (negative controls) and defects press-fitted with different PTMC/HA composites and the skin was closed with a continuous pattern of Monocryl 3.0. Post-operative analgesia was administered to the animals (Carprofen $4 \mathrm{mg} / \mathrm{kg}$ s.c. q $24 \mathrm{hrs}$ for $72 \mathrm{hrs}$; buprenorphine $0.1 \mathrm{mg} / \mathrm{kg}$ i.m. $8 \mathrm{hrs}$ for $24 \mathrm{hrs}$ and Fentanyl transdermal patches $12 \mu \mathrm{g} / \mathrm{h}$ for $72 \mathrm{hrs}$ ) and they were kept single in a cage for the entire study. MicroCT 
scanning of every animal was performed under general anaesthesia (as described above) postoperatively (day 0), after 3 weeks and after euthanasia (6 weeks), using a highresolution peripheral quantitative computed tomography (HR-pQCT) operated at $59 \mathrm{kVp} /$ $900 \mu \mathrm{A}$ with a nominal resolution of $82 \mu \mathrm{m}$ (SCANCO Medical AG). Injection of fluorochromes was performed in order to observe new bone formation, by injecting calcein green and xylenol orange after 2 and 4 weeks of surgery respectively. Euthanasia of the animals was realized after 6 weeks by intravascular injection of $600 \mathrm{mg}$ Pentobarbital.

\subsubsection{Histology and histomorphometric evaluation}

Following euthanasia, the calvaria including the four defects, without soft tissues, was resected from the skull and stored in methanol $70 \%$ for histology analyses. Undecalcified sections were obtained after methylmethacrylate embedding and stained using GiemsaEosin. Histomorphometric evaluation and semi-quantitative analyses were conducted by an anatomopathologist, blinded, taking into consideration the inflammatory response, the osteotomy content (nature and composition of the neo-formed tissue), the vascularization and the osseointegration of the scaffolds.

\subsection{Statistical analyses}

Statistical analysis of data was performed using Prism software (GraphPad Software, La Jolla, CA, USA). We assumed normal distribution of data. One way ANOVA with Tukey's multiple comparison test was applied to detect significant differences between experimental groups (with $\mathrm{p}<0.05$ ). Data presented are means \pm standard deviation $(\mathrm{SD})$ unless stated otherwise.

\section{Results}

\subsection{Fabrication and Characterization of disparate PTMC/HA scaffolds}

Resins (see supplementary table 2 for description of the formulations) were optimized for fabrication of scaffolds of photo-crosslinked PTMC and disparate PTMC/HA using stereolithography technique (SLA). Composite scaffolds of PTMC combined with 0, 20 and 40 wt. \%. HA, labelled respectively as PTMC, PTMC 20 and PTMC 40, were SLAfabricated.

Macroscopically, the SLA-fabricated scaffolds resembled the designed scaffold model, with a highly interconnected and gyroid-like porous structure (Figure 1a and b). Using SEM, Figure 1c, a smooth and regular surface was observed on PTMC scaffolds. However, on the surface of PTMC 20 and more importantly on PTMC 40 scaffolds, agglomerates of HA particles were observed, creating a topographical roughness on the surface of the built structures. The surface topography of PTMC 20 and PTMC 40 implants is relatively complex, with pores and undercuts associated with nano/micro-roughness, which are architectural prerequisites for de novo bone formation[24].

This difference of roughness and asperity was also noticeable using microCT (Figure 1d). From the 3D reconstructions, the built internal architecture demonstrated a high degree of 
reproduction and homogeneity for the PTMC and PTMC 20 resins as seen in the cartography of the trabecular thickness (strut thickness) and trabecular space (pore diameter) (supplementary figure $1 \mathrm{a}$ and $\mathrm{b}$ respectively). The incorporation of $20 \mathrm{wt} \%$ of $\mathrm{HA}$ in the PTMC, resulted in a slight increase in the strut thickness (figure 1e and supplementary figure 1a) and a decrease of the average pore size (figure $1 \mathrm{f}$ and supplementary figure $1 \mathrm{~b}$ ) correlated with a decreased volume of porosity (figure $1 \mathrm{~g}$ ). These micro-architectural investigations revealed that the addition of $40 \mathrm{wt}$ \% of HA in PTMC resulted in a more heterogeneous structure with lower internal fidelity compared to the other resins.

a) Designed model
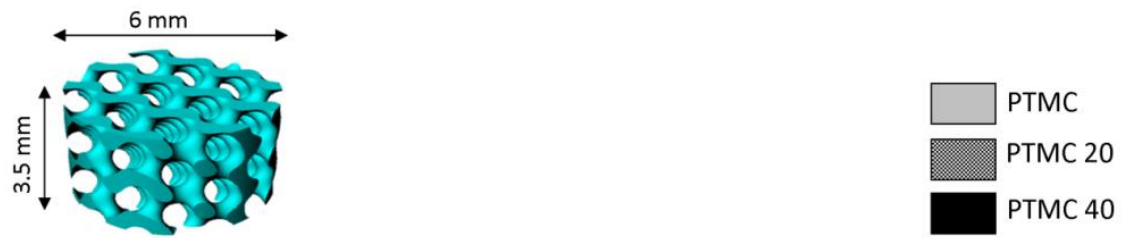

b) Macroscopic

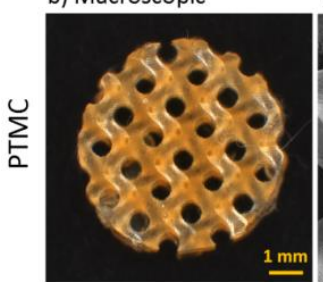

c) SEM
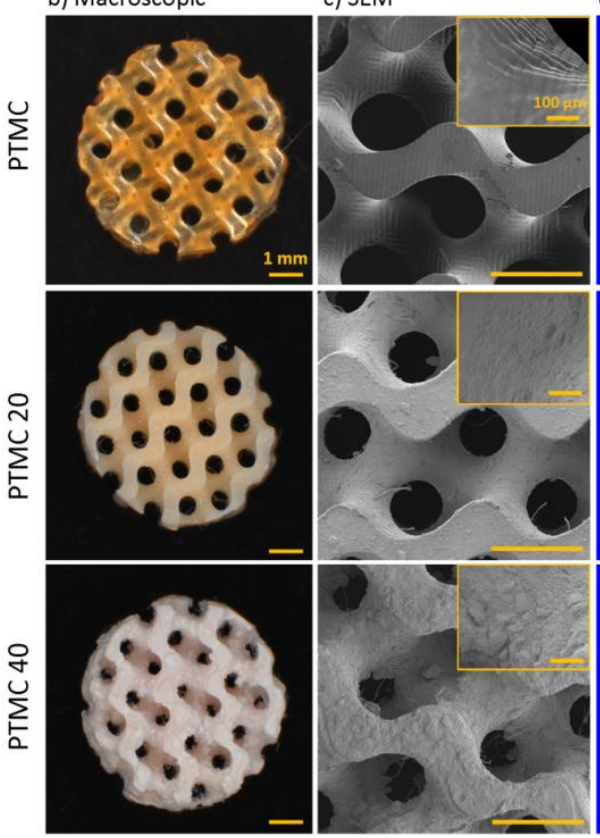

d) 3D Reconstruction
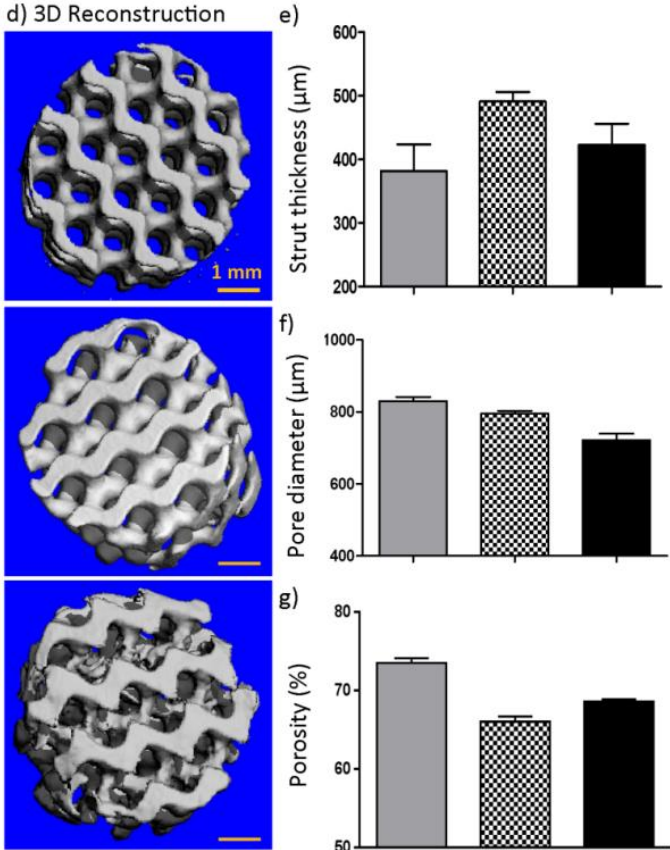
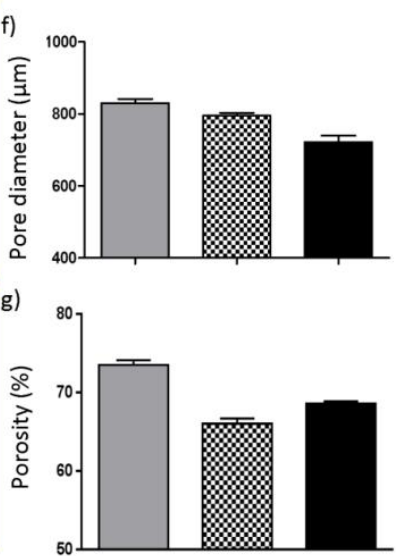

Figure 1. Macro-porous scaffolds of PTMC fabricated by stereolithography with varying amounts of HA. a) Model design processed using k3dsurf and Rhinoceros_software (exported as .STL-file, Standard tessellation language) and b) macroscopic and c) microscopic SEM observation of the different SLA-fabricated scaffolds (insets represent a magnification of the respective surfaces). CT monitoring of $d$ ) the $3 D$ architecture and determination of $e$ ) the strut diameter, $f$ ) the pore diameter and $g$ ) the volume of porosity for PTMC, PTMC 20 and PTMC 40 scaffolds. 
To conclude, by selecting an appropriate resin formulation and printing process, we were able to manufacture scaffolds with various amount of HA loaded into the PTMC, which exhibit reproducible macro- and microscopic features suitable for bone tissue engineering application[22].

SEM analyses performed on the surfaces of the SLA-fabricated scaffolds clearly showed a dense HA layer covering PTMC 20 (Figure 2a and b) and PTMC 40 (data not shown). The needle-like nano-HA particles exhibit a random spatial alignment and agglomeration, responsible for the singular topography previously described figure 1c and supplementary figure 2. The spatial repartition of the HA particles was investigated on SEM using backscattered electrons (BSE) mode, revealing a clear surface enrichment of $\mathrm{Ca}$ and $\mathrm{P}$ elements on PTMC 20 and PTMC 40 (Figure 2c and d), with approximate thicknesses of 10 and $30 \mu \mathrm{m}$ respectively. The quantitative analyses, Figure $2 \mathrm{e}$, demonstrated the global increase of Ca and P from PTMC 20 to PTMC 40 scaffolds, on both the bulk materials and the surfaces. However, significant enrichment of $\mathrm{Ca}$ and $\mathrm{P}$ elements was noted cortically, on both groups, in comparison to the bulk portion. Importantly, the presence of $\mathrm{C}$ element (originating from polymeric matrix) apposed to $\mathrm{Ca}$ and $\mathrm{P}$ in the corralling layer suggests that HA nanoparticles are physically embedded in PTMC matrix and are not only loosely agglomerated.

Additionally, high resolution tomography, figure $2 \mathrm{f}$, illustrated the formation of a thin and almost continuous microscale layer of tightly agglomerated HA nanoparticles on the disparate PTMC/HA scaffolds (which could not be observed using microCT 40 (supplementary figure 1) due to limitation of its resolution).

Subsequently, other physico-chemical characterizations were performed on PTMC/HA scaffolds in order to evaluate the additional changes triggered by HA incorporation. We noted that PTMC scaffolds tend to shrink following incubation in PBS, while PTMC 40 swells to $120 \%$ of its original size (figure 3a). This significant difference can be partly explained by the increased wettability of the structures containing high amount of HA, as shown by the contact angle measurement. An increasing load of HA was also correlated to an increasing protein surface adsorption (figure $3 \mathrm{~b}$ ), with dramatic augmentation for PTMC 40 compared to PTMC and PTMC 20. The presence of HA loaded in PTMC 20 and 40 allowed free $\mathrm{Ca}^{2+}$ to be continuously released in vitro. The main differences comparing both groups is the higher initial burst release of $\mathrm{Ca}^{2+}$ observed for PTMC 40 (figure 3c). 

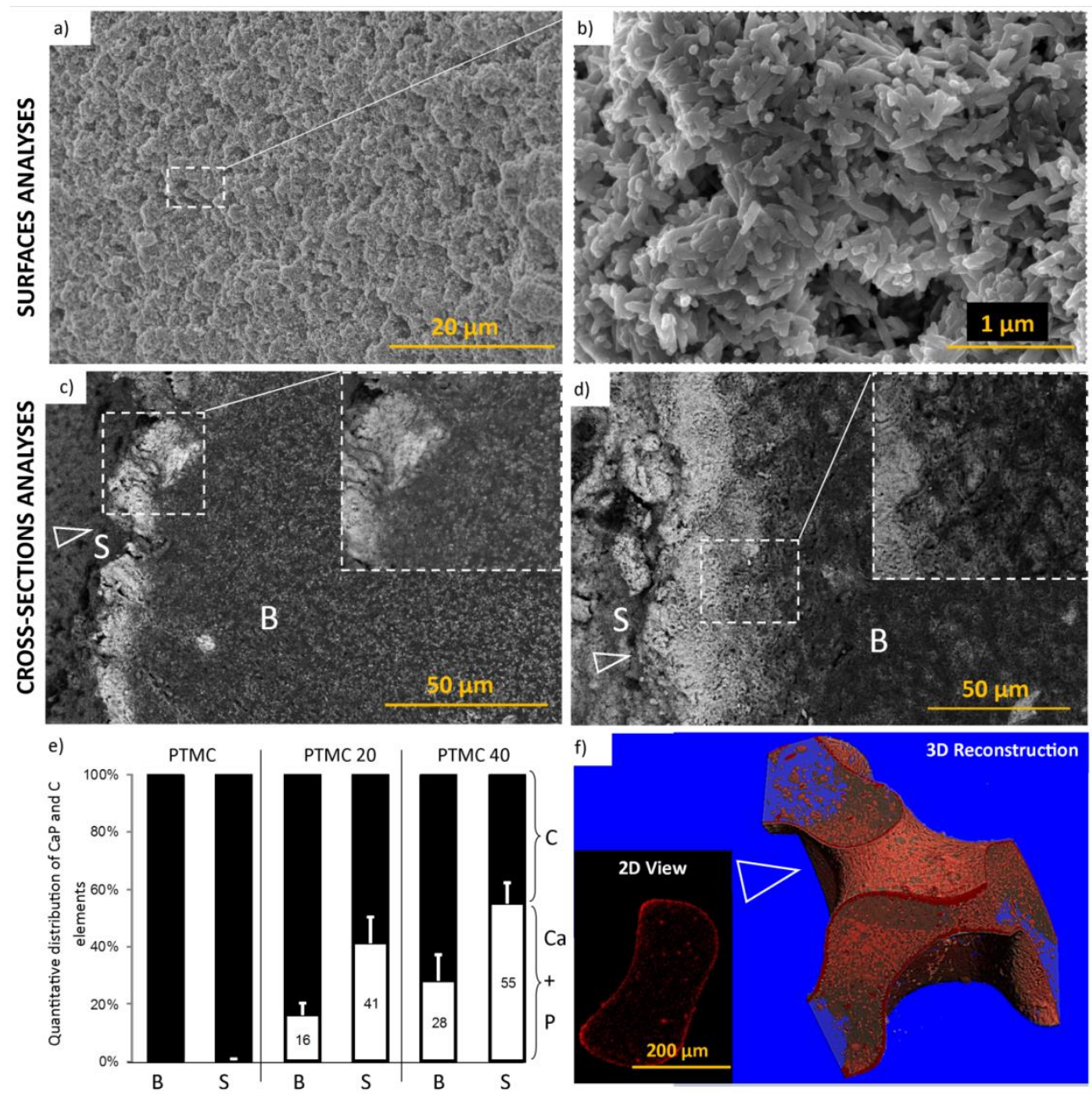

Figure 2. Surface-enrichment of HA in PTMC scaffolds occurring during stereolithography processing. Illustration of the layer of agglomerated HA particles formed during SLA process covering the surface of the composite scaffolds, by SEM analysis of the surface ( $a$ and $b$, illustrating the random spatial organization of the needle-like shape HA nanoparticles on PTMC 20) and by BSE-SEM analysis performed on c) PTMC 20 and d) $P T M C 40$ (cross sections illustrating the presence of $\mathrm{Ca}+P$ (shown in light grey) dispersed in PTMC (C element is represented in dark grey)).e) Quantification of the $C a+P$ and $C$ elements at the surface or in the bulk ( $S$ and $B$ respectively) for the different scaffolds (measured on at least 6 different spectra) and f) high resolution microtomography reconstruction (illustrated only for PTMC 20), with high-contrasted area coloured in red (revealing HA-agglomerates repartition). White triangles point the dense HA-rich domains covering the scaffolds' surface. 

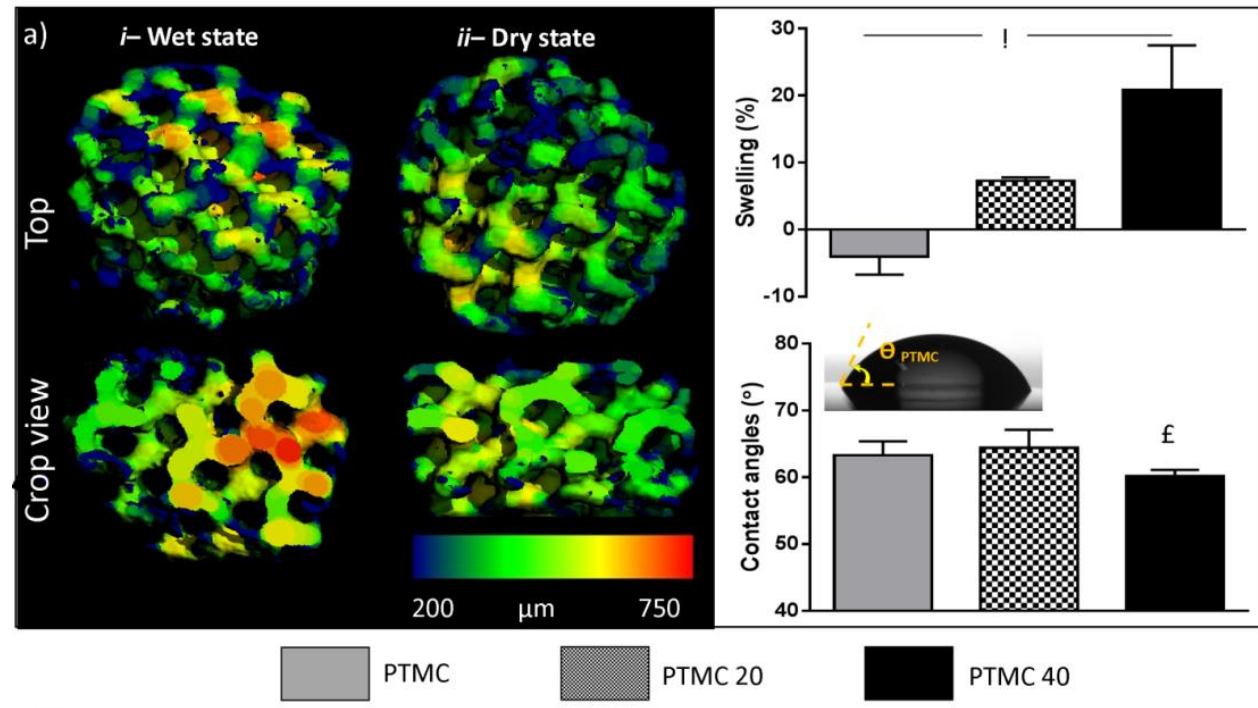

b)

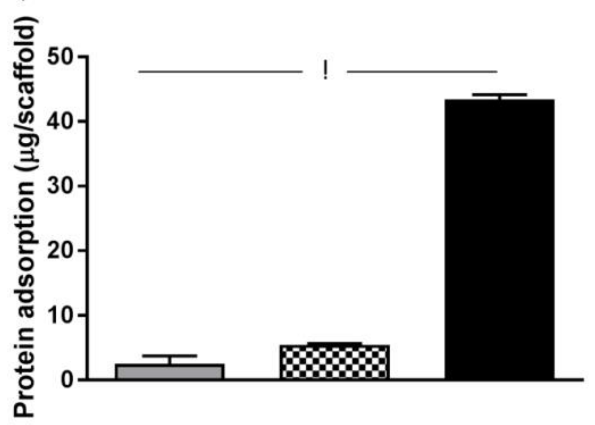

c)

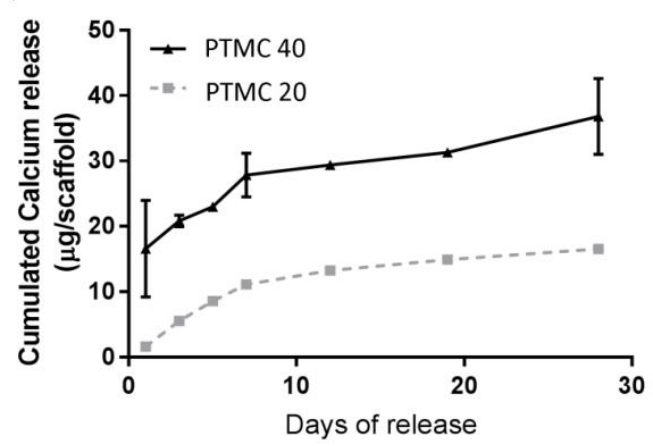

Figure 3. Incorporating HA to PTMC scaffolds triggers fundamental physico-chemical alterations essential for promoting bone healing. a) Swelling behaviour illustrated by the cartography of the trabecular thickness distribution following 28 days of incubation in PBS i- before drying and ii- after drying (top view and crop view represented for PTMC 40), quantification of the swelling propensity for the different scaffolds correlated with the wettability (contact angle measurement) (! and £ indicate significance for the three groups or only for PTMC 40 respectively). b) Determination of protein absorption on the different scaffolds following 5 days of incubation in PBS-containing serum estimated using Bradford Protein assay (! indicates significance for the three groups). c) Analysis of the calcium release profile from the different scaffolds in PBS at $37^{\circ} \mathrm{C}$.

\subsection{Cell proliferation and matrix deposition}

The estimated number of cells per scaffold 1 day post-seeding, figure 4a, suggested that the addition of HA into the PTMC positively impacted the cells attachment to the scaffolds, as the DNA values significantly and gradually increased from pure PTMC to PTMC 40. Within 28 days of incubation all of the scaffolds permitted significant cell growth, as shown figure $4 \mathrm{a}$ and cells colonized the entire porous network of the scaffolds (figure $4 \mathrm{c}$ and d). However, considering the proliferation rate (taking into account the variation in the 
cell adhesion, figure 4a), we did observe that combining HA to PTMC resulted in a decelerated cell division (figure $4 b$ ).
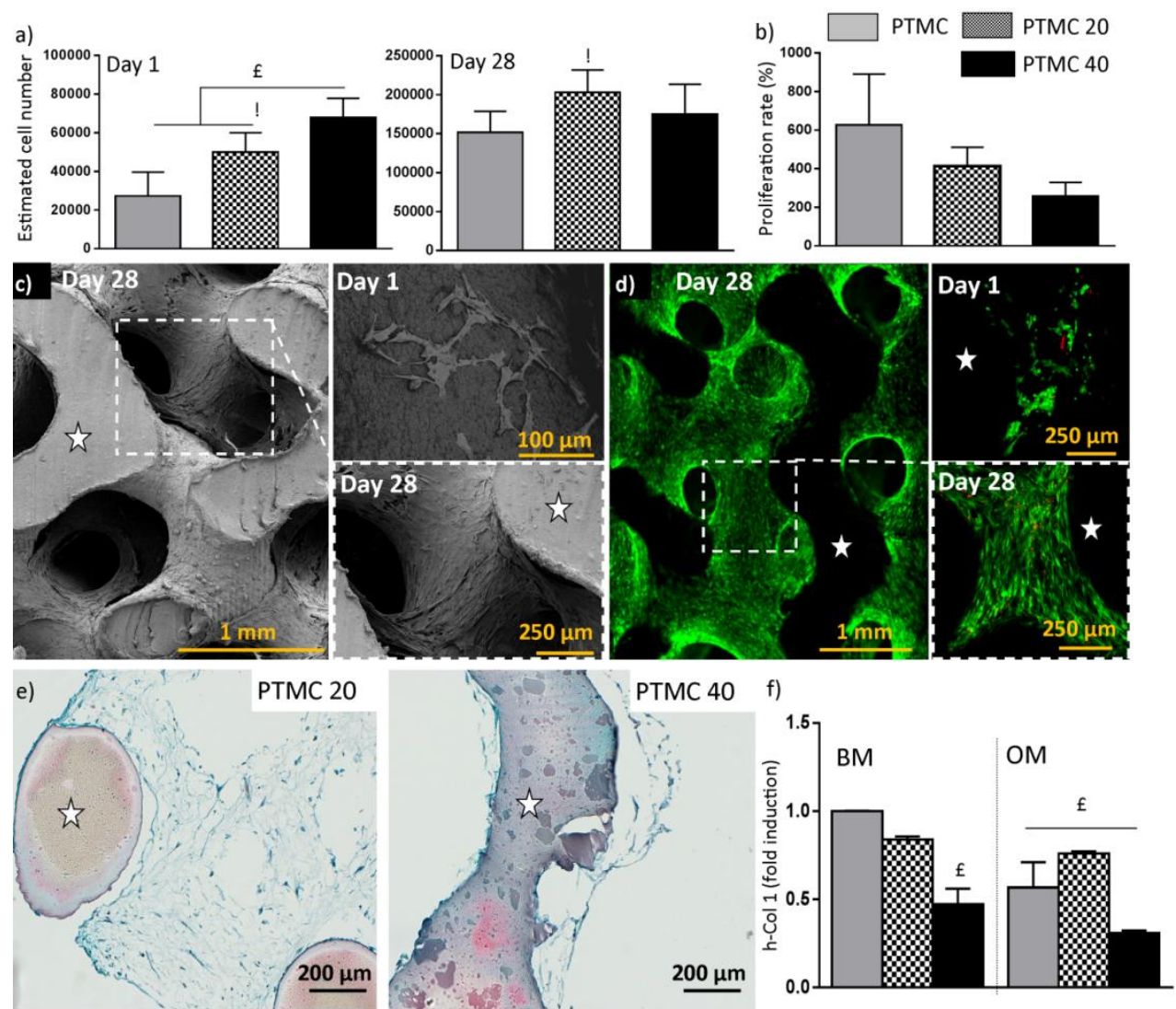

Figure 4. Loading HA in PTMC scaffold impacts in vitro cell adhesion, proliferation and matrix deposition. a) Estimation of scaffolds colonization by hBMSCs from day 1 to day 28 (estimated by DNA assay quantification, hBMSCs having approximately $10.5 \mathrm{pg}$ of DNA per cell, ! and £ reported significance for PTMC 20 and PTMC 40 respectively compared to PTMC) and b) representation of the proliferation rate for the different scaffolds. c) SEM observation and d) Live/Dead ${ }^{\otimes}$ staining of scaffold's cross-section at 1 and 28 days post-seeding (illustration for PTMC 20, from z-stack acquisitions at $2.5 \mathrm{X}$ and $10 \mathrm{X}$ ). e) Histological Safranin-O Fast Green staining illustrating in blue the collagen fibres present in the ECM deposited by hBMSCs after 28 days of cultivation in OM (showed for PTMC 20 and PTMC 40, at 10 X.). f) Type I human collagen gene expression analysed after 14 days of hBMSCs cultivation on the different scaffolds in basal and osteogenic media (BM and OM respectively, $£$ reported significance compared to PTMC in BM). White stars present in $c, d$ and e denote scaffold's crosssection.

hBMSCs secreted a collagenous-rich matrix through the porosity of PTMC (data not shown) and PTMC 20 scaffolds (figure 4e). Histological observation revealed that cells proliferating in PTMC 40 scaffolds produced collagen as well, but in a reduced extent compared to PTMC and PTMC 20. Gene expression analysis corroborated this observation, 
as h-Col I gene was also found to be down-regulated following 14 days of cultivation, in both conditions, for PTMC 40 compared to PTMC and PTMC 20 (figure 4f).

\section{3. hBMSCs osteogenic differentiation}

The ALP activity was significantly increased for both PTMC 20 and PTMC 40 compared to PTMC surface from day 14 to day 21 in OM media (figure 5a). The presence of HA did influence the synthesis and the activity of ALP protein. Different tendencies could be observed from the investigation of the gene expression encoding for ALP. At day 14, hALP was significantly up-regulated only for PTMC 40 and not for PTMC 20. Indeed, hALP expression for PTMC 20 was similar to the control (PTMC scaffold without HA in BM condition, figure 5b), whereas ALP activity (corresponding to the activity of the protein synthesised) was shown to be significantly increased compared to PTMC only (figure 5a). Such discrepancy could reveal that ALP gene expression (which is an early marker for osteogenic differentiation) at day 14 for cell proliferating on PTMC 20 has already declined, as its peak, in terms of intensity of gene expression, precedes chronologically the peak of the encoded protein synthesis.

Calcium deposition was subsequently investigated using alizarin red staining (ARS) and quantification (figure $5 \mathrm{c}$ and $\mathrm{d}$ ). The positive red staining revealed that the deposition of calcium increased over time in all surfaces, but more strongly on PTMC 20 and PTMC 40 after 28 days of incubation. The staining quantification confirmed the enhanced mineralisation on the films containing HA compared to the TCPS and PTMC surfaces at 28 days. Interestingly, higher ARS values were obtained on the PTMC 20 resin compared to the PTMC 40 for the two donors. Additionally, SEM observations, figure 5d, corroborated that hBMSCs mineralization was triggered by HA in PTMC, whereas a limited number of inorganic clusters (artificially coloured in red) could be distinguished on cell monolayers grown on PTMC and TCPS control surfaces. 


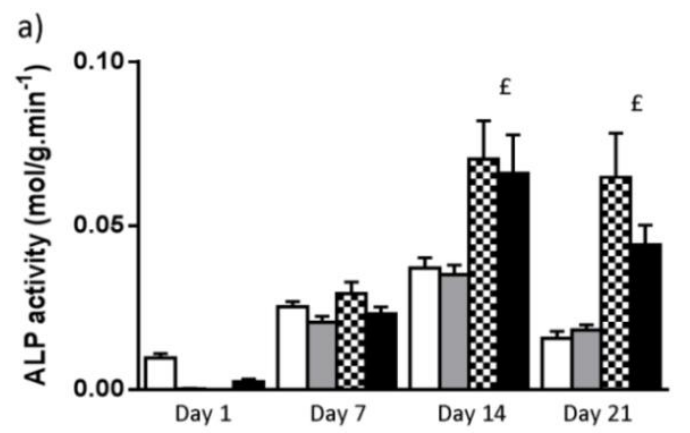

b)
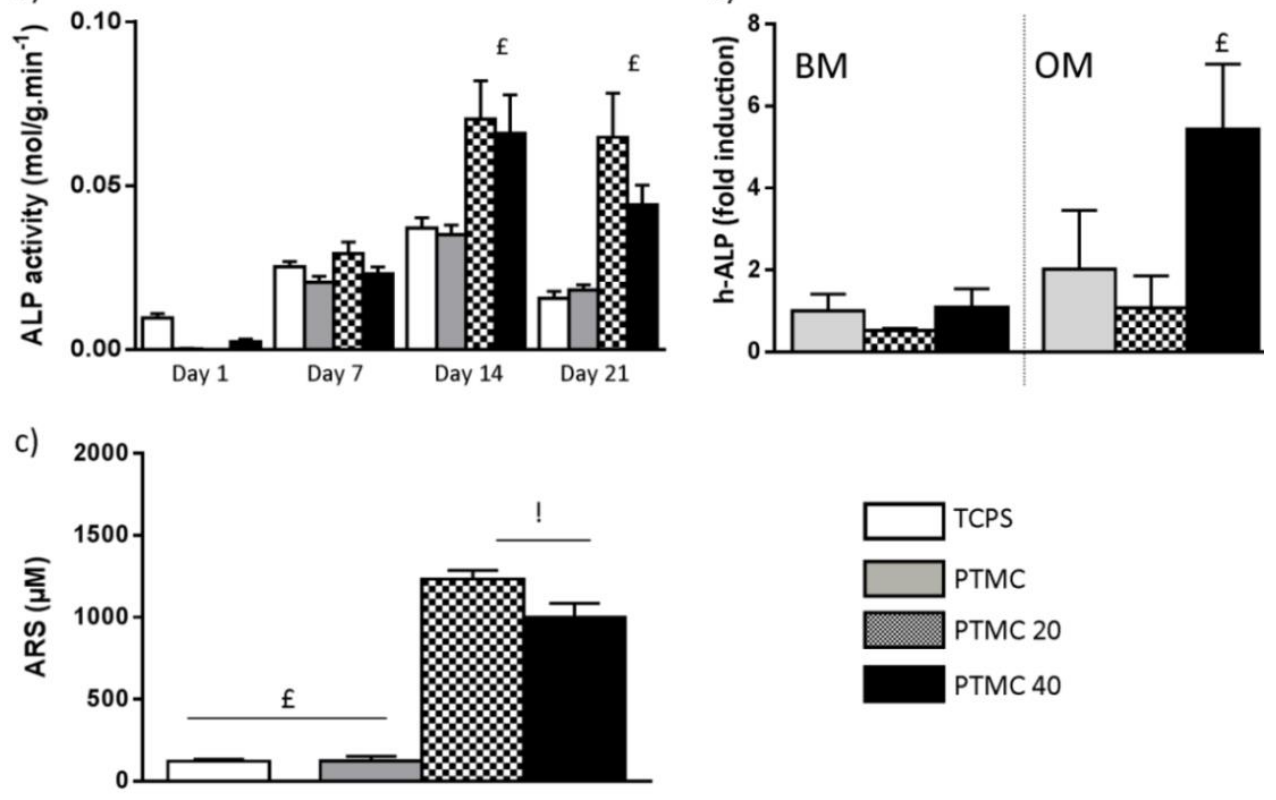

d)
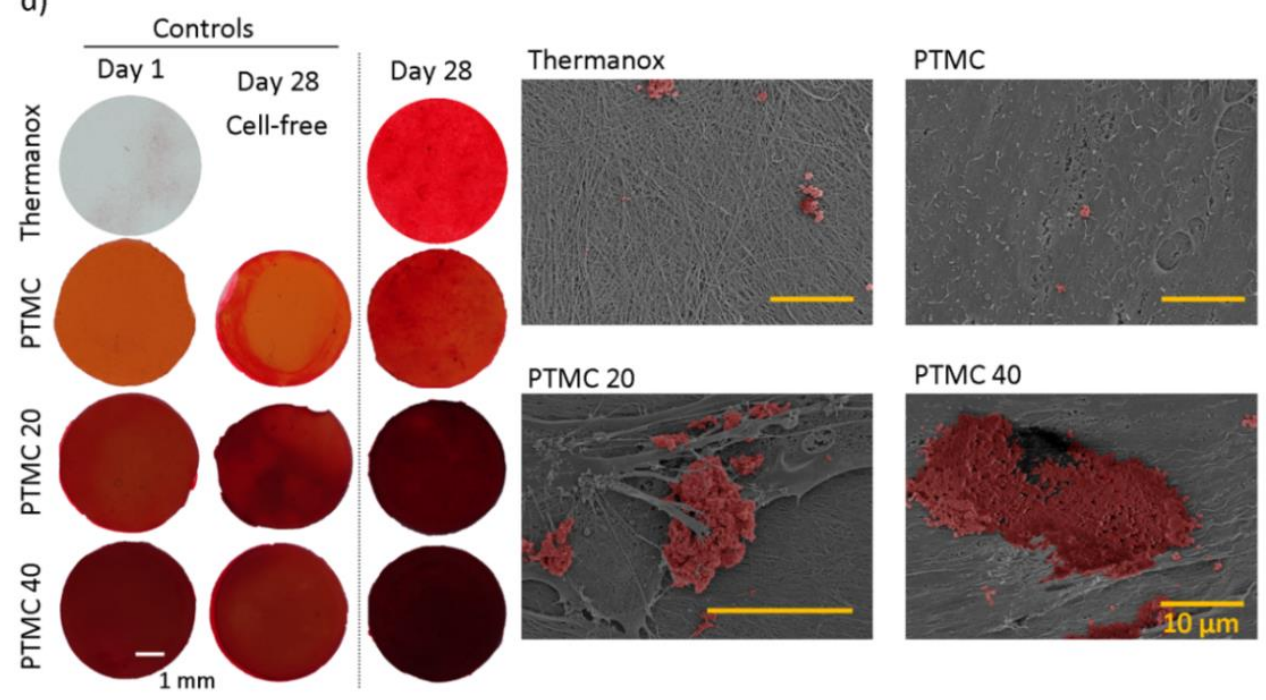

\section{PTMC 20}
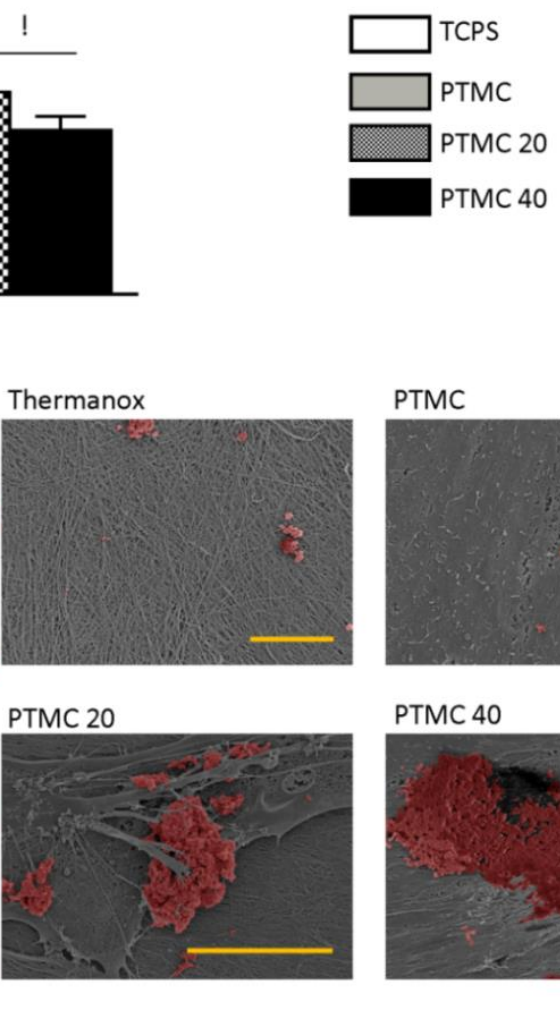

\section{PTMC 40}

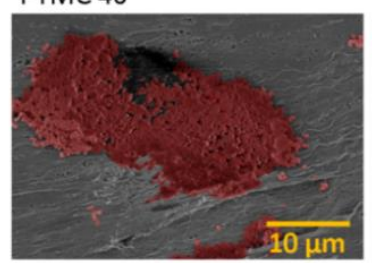

Figure 5. Combining HA to PTMC exacerbates osteogenic differentiation of hBMSCs under OM condition. Influence of PTMC scaffold composition on a) ALP activity, b) $h$-ALP gene expression (analysed after 14 days of hBMSCs cultivation on the different scaffolds in basal and osteogenic media (BM and OM respectively), $£$ reported significance compared to PTMC) and c) Alizarin Red Staining (ARS) quantification at day 28 (with $£$ indicating significance between PTMC/HA and controls and! indicating significance between PTMC 20 and PTMC 40) (ARS corrected by the background O.D. values due to the composition of the films at day 1) (a and c represent average values \pm standard error of the mean). d) Illustration of the intensity of ARS for the different controls and film materials and of calcium deposition on the cell monolayer following 28 days of cultivation on the different surfaces (SEM images with mineralization highlighted by artificial red colouring using Photoshop ${ }^{\circledR}$ ). 


\subsection{In vivo bone formation}

The SLA scaffolds were assessed using a calvarial defect model and bone ingrowth monitored using in vivo microCT at 0,3 and 6 weeks with empty defects as control group. Selected scaffold architecture with a high degree of porosity (volume of porosity $70 \%$ and pore diameter of $700+/-100 \mu \mathrm{m}$ ) showed to enable fast angiogenesis following implantation (supplementary figure 3 ).

Bone ingrowth was observed after three weeks for all the groups and continued until week 6, with clear improvement for defects filled with PTMC 20 and 40 (figure 6a). As $6 \mathrm{~mm}$ large defect is considered to be non-critical size on rabbit calvarium model, small amount of new bone was formed for the empty groups but without reaching a full bridge over the 6 weeks of the experiment (figure $6 a$ and $b$ ).

The quantification of new bone formation, figure $6 \mathrm{~b}$, showed additionally that scaffolds based on PTMC 40 significantly enhanced early bone tissue formation in the defect at week 3 and maintained higher bone content at week 6 compared to all the other groups. Noteworthy, PTMC 20 exhibited also a positive and significant effect on bone ingrowth, but only at 6 weeks compared to the empty defects.
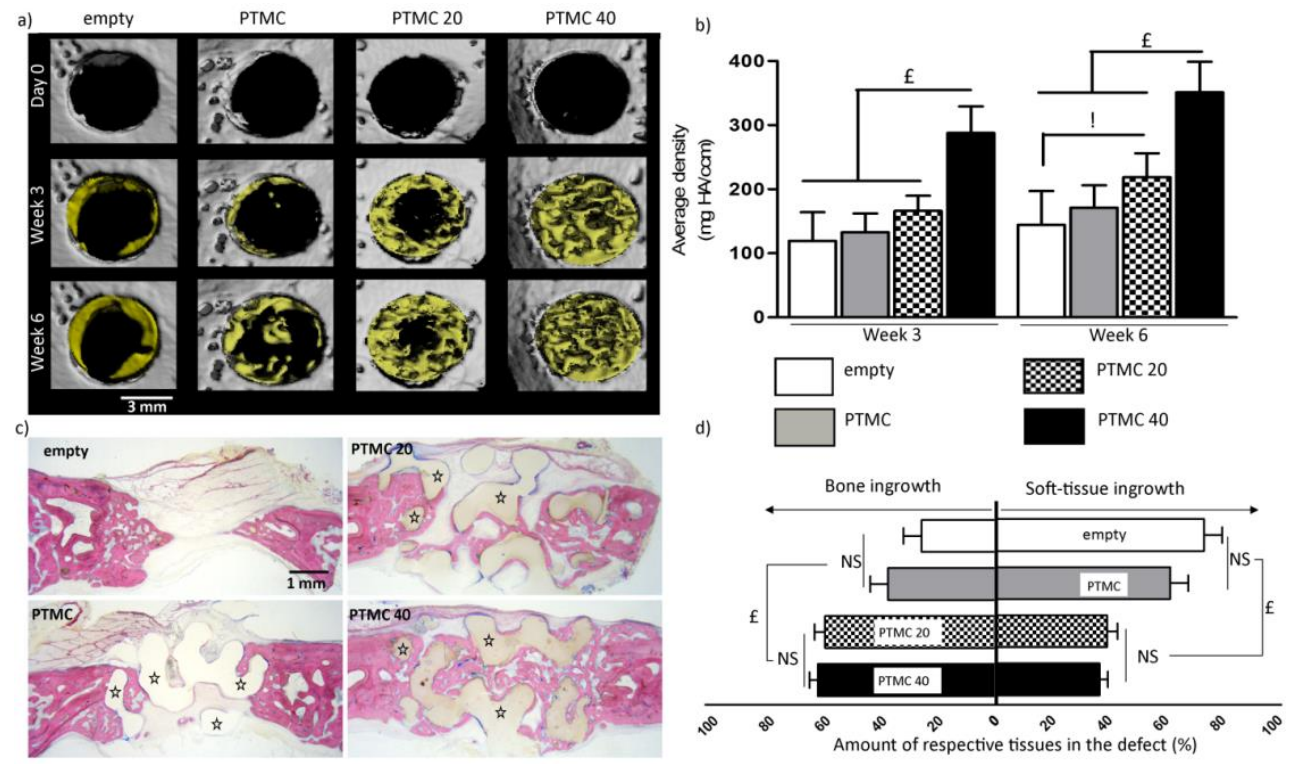

Figure 6. PTMC/HA stimulates in vivo mineralization and bone formation. a) Representative $3 D$ reconstructions from the microCT images showing the native calvarial bone (in grey) and the new bone (in yellow) (the intrinsic signals of the scaffolds were subtracted for all the time points). b) Quantification of the new bone formation at week 3 and 6 estimated by the average density of the defects (corrected by the postoperative values due to the intrinsic signal of the scaffolds (day 0), with! and $£$ indicating significance compared to PTMC 20 and 40 respectively). c) Giemsa-Eosin staining revealing the new bone formation after 6 weeks of implantation (bone is stained in pink and soft tissue appeared in slight brown, stars denote scaffolds' cross-section) and d) histomorphometric semi-quantification of bone versus soft tissue in the defect for the different groups ( $£$ indicated significance compared to PTMC 20 and 40). 
Histological analysis, figure $6 \mathrm{c}$ and $\mathrm{d}$, showed that the majority of the tissue filling the defects was composed of soft tissue for the empty group and PTMC, with an estimated bone volume in the defect of only 26 and $38 \%$ respectively at 6 weeks. In comparison, around $60 \%$ of the PTMC 20 and PTMC 40 scaffold porosities were filled by bone with no significant difference between both groups. By comparing both histological and microCT techniques, we can conclude that the amount of bone growing in PTMC 20 is not significantly different from PTMC 40 (figure 6d), but the bone seems more mineralized and mature in PTMC 40 (higher density values in microCT, figure 6b).

Injection of calcein green and xylenol orange fluorochromes after 2 and 4 weeks of implantation brought additional insight regarding the bone healing kinetic. Indeed, from the fluorescence staining, figure 7 , two different bone ingrowth mechanisms are discernible depending on the scaffolds' composition. For the empty and PTMC groups, bone ingrowth started from the calvarial edges of the defect toward the center of the defect. In this study, bone deposition distribution was materialized by the sequential positioning of the fluorochromes, with a first green-stained front localized close to the edges of the defect, representing new bone formed at 2 weeks, and then a second red-stained front present in a more central area of the defect or of the PTMC scaffold for the new bone created at week 4. This phenomenon, corresponding to a distance osteogenesis[25], was observed in $100 \%$ of the histological sections (8 out of 8) for the PTMC group.

In opposition, adding HA into PTMC matrices directly influenced the bone healing cascade. Indeed, we observed a contact osteogenesis mechanism for PTMC 20 and PTMC 40 , characterized by a direct bone apposition to the surface of the scaffolds growing toward the porous space compartment regardless the distance of the scaffold surface to the host bone bed (figure $7 \mathrm{~b}$ and c)[25]. This pattern of new bone formation was observed in 5 (63 $\%)$ and $8(100 \%)$ out of 8 histological sections for PTMC 20 and PTMC 40 respectively. New bone was already formed at week 2 in the central part of PTMC 20 and, more importantly, of PTMC 40 scaffolds (depicted figure $7 \mathrm{~d}$ by calcein green area quantification). PTMC scaffolds without HA allowed bone colonization, but mainly visible from the peripheral areas, following 4 weeks of implantation, figure $7 \mathrm{~b}, \mathrm{c}$ and $\mathrm{d}$.

When looking closely at the interface between the surface of the biomaterials and the neoformed tissue (bone-bonding), we observed unequivocally a significant improvement regarding the osseointegration of the scaffolds containing $40 \mathrm{wt} \%$ HA compared to PTMC with 20 wt.\% HA (figure 7e). Indeed, the degree of bone anchorage to the scaffolds was enhanced by a factor 1.4 for PTMC 40 compared to PTMC 20, meaning that an average of 60 to $80 \%$ of the surface of the PTMC 40 scaffolds were in direct contact with newly formed bone. Finally, a higher degree of bone tissue maturity was achieved in the porous network of PTMC 20 and PTMC 40, with marrow spaces and an intense vascularization, in comparison to PTMC scaffolds (supplementary figure 3). 


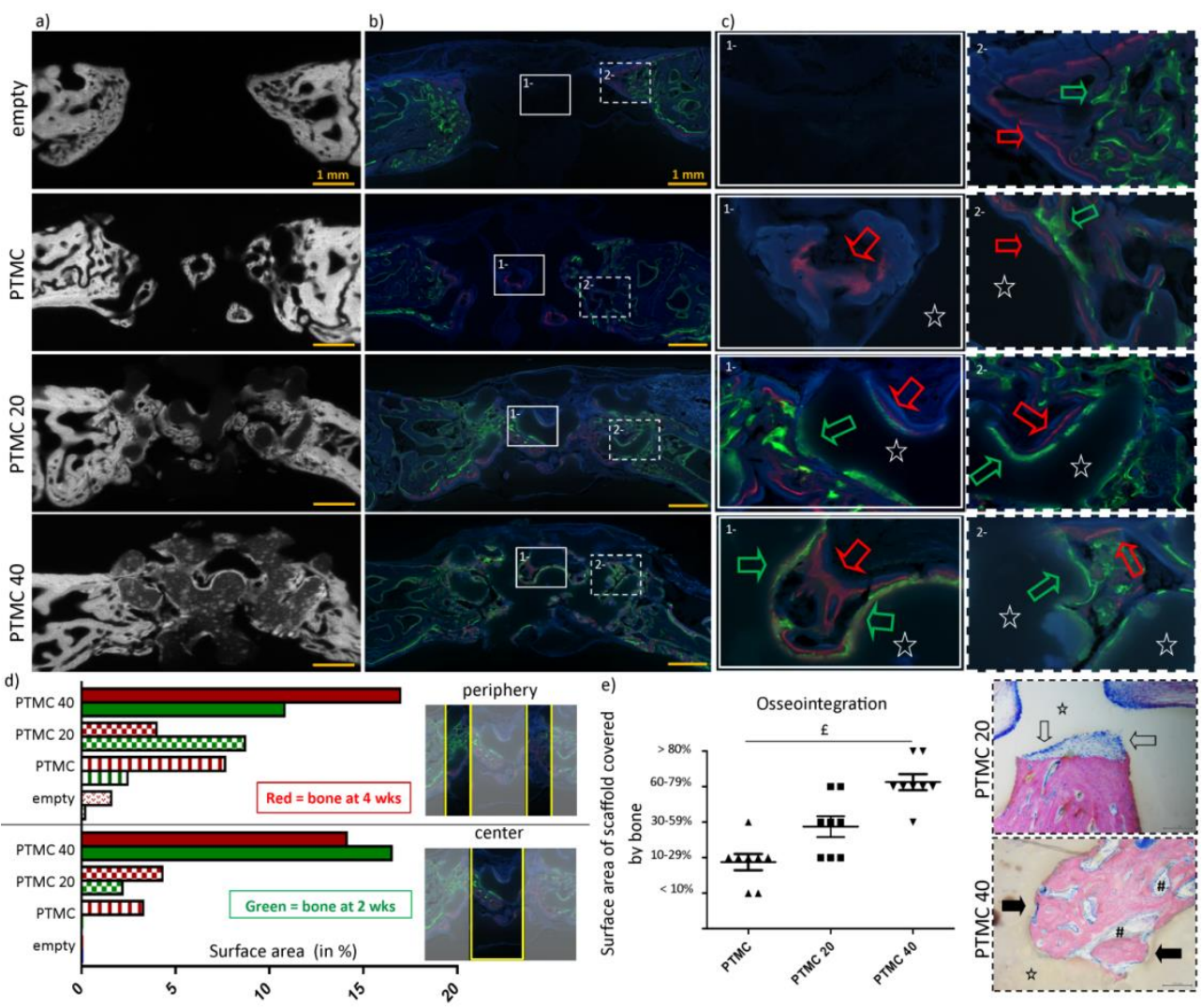

Figure 7. Osteoconductive HA directly governs PTMC composite implants osseointegration by contact osteogenesis and de novo bone formation. a) Contact radiographs of the defects correlated with b) fluorescence imaging of new bone formed following 2 weeks (in green) and 4 weeks (in red) of implantation. c) High magnification micrographs of 1 - central and 2-peripheral regions of the defect highlighting the different osteogenesis mechanisms (i.e. distance osteogenesis versus contact osteogenesis observed for PTMC and PTMC/HA respectively). Stars denote the biomaterial cross-sections, green and red arrows represented the bone formed after calcein green and xylenol orange injection respectively. d) Zonal histomorphometric illustration of bone healing kinetic by quantification of green-labelled and red/orange-labelled areas (representing new bone formed at 2 and 4 weeks respectively in the central and peripheral area (each of $7.7 \mathrm{~mm} 2$, expressed in percentage relative to the available porous space) of the images represented figure 6b, using Photoshop ${ }^{\circledR}, n=$ 1/group). e) Representation of different degree of bone-bonding scaffolds with semi-quantitative histomorphometric estimation of the degree of scaffold anchorage to the newly formed bone ( $£$ indicated significance between the three groups, $n=8 /$ group). Stars denote scaffold biomaterials, empty arrows show surface biomaterial in contact with soft tissue whereas black arrows depict direct bone integration, hashtags point out the marrow tissue.

\section{Discussion}

\subsection{Biological response of the disparate PTMC/HA scaffolds}

Naturally, bone healing cascade is initiated by the migration and then the differentiation of progenitor osteogenic cell population[26]. Therefore, it is essential to investigate the influence of scaffold's properties on those earliest biological events. In order to mimic such 
in vivo phenomenon, human primary stem cells isolated from bone marrow compartment were employed for the in vitro adhesion, proliferation and osteogenic differentiation investigations.

The in vitro attachment study showed that PTMC 40 allowed more robust cell adhesion compared to PTMC and PTMC 20 (figure 4). The modification of the cell adhesion parameter is likely to result from the topographical and chemical surface alteration observed on PTMC 40 surfaces (supplementary figure 2). Being able to modulate this parameter by varying HA content could have triggered the positive bone healing outcomes observed in PTMC/HA, as several authors demonstrated that osteoprogenitor cells start to attach and to colonize biomaterial scaffolds within one day of implantation[27, 28]. Interestingly, the proliferation rate over 28 days of culture was the lowest in this same PTMC 40 group. In fact, it was largely reported that surface roughness negatively impacts cell proliferation, which correlates to a more differentiated osteogenic cell phenotype[28]. Our findings corroborate this hypothesis, as the in vitro osteogenic markers (ALP and mineralization) were increased in PTMC containing HA compared to pure PTMC (figure 5). Surprisingly, both collagen deposition along with type I collagen gene regulation were decreased in PTMC 40 compared to PTMC and PTMC 20. However, this is not an isolated finding, as other publications have observed that osteogenic differentiation of MSCs in ceramics did not always result in Col-I gene upregulation[29]. Chronologically, the matrix secreted by osteogenic cells during de novo endosseous implant healing is composed of non-collagenous proteins (called cement line[24]), which could explain the difference observed in vitro between PTMC and PTMC 40 (figure 4).

Following implantation of composite PTMC scaffolds in rabbit calvarial defect model, a significant amount of new bone ingrowth through the entire porosity of the PTMC 20 and PTMC 40 scaffolds was observed. In comparison, mainly soft tissue was detected in the control PTMC group. In terms of quantification of new bone formation, PTMC 20 performed as well as PTMC 40, but the osseointegration quality of the implants (looking at the focal interaction between the biomaterials and the tissue) correlated with the highest amount of HA.

Noteworthy, the kinetic of new bone ingrowth in vivo investigated using fluorochromes staining revealed that the amount of HA into the PTMC matrix directly governed the osteogenesis mechanism (figure 7).

De novo bone formation was detected on the surface of PTMC 20 and more importantly, on PTMC 40, due to the osteoconductivity of the composite scaffolds.

In comparison, bone formation for PTMC only resulted exclusively from distance osteogenesis, starting from the edges of the calvarial defect toward the center. In implantology, contact osteogenesis is the result of an implant allowing for "osteoconduction", meaning that its surface favours osteogenic cells to migrate and to attach to its surface (importance of fibrin clot and implant micro-topography as previously mentioned). Next in order, a new mineralized tissue similar to a cement line (occurring naturally in bone fracture healing) is formed at the interface implant/tissue, which is called 


\section{Chapter 7}

"de novo bone" formation. To summarize, the main differences between contact osteogenesis and distance osteogenesis is the formation of the cement line ("de novo bone formation") covering directly the surface of the implant in the first scenario.

Those different osteogenesis mechanisms, extensively described by Davies et al.[25, 30, 31], have a direct impact on the quality of implant endosseous integration (as seen figure 7e).

Indeed, as observed by Puelo et al., bone growing rate occurring through contact osteogenesis is $30 \%$ faster than in distance osteogenesis[32]. Our findings corroborate this phenomenon, as we clearly noted, figure $7 \mathrm{~d}$, more bone formed at 2 and 4 weeks in PTMC 40 (either in the periphery or in the center of the defect).

\subsection{Correlation between PTMC/HA fabrication process and biological properties}

In order to unravel underlying mechanisms toward biofunctionality of the scaffolds, several physico-chemical characterizations of the scaffolds have been undertaken. Importantly, increasing the volume fraction of HA particles loaded into the PTMC resin resulted in a dense $\mathrm{CaP}$ cortical layer, observed on the surface of the composite stereolithography scaffolds (figure 2). Indeed, it is known that the stability and segregation of nanoparticles in a polymer/solvent mixture is governed by the nature of the interactions between the blend and the particles (Van Der Waals, electrostatic, hydrophobic-hydrophilic, etc.).

In the selected stereolithography fabrication process, the nature of the polymeric phase evolved from soluble macromers of PTMC-MA to photo-crosslinked chains of PTMC. This chemical modification directly impacts on the polymer's radius of gyration and its chain stretchability. Taking into consideration that spatial distribution of nanoparticles can be controlled depending on the size of the nanoparticles relative to the radius of gyration of the polymer[17], we hypothesise that a fraction of HA particles are expelled to the surfaces during/after the photo-crosslinking reaction. Additionally, as more crosslinks are formed in the polymer network during photoreaction, conformational entropy in the system naturally decreases. To regain a certain degree of entropy (and in this way polymer chains do not have to stretch around nanoparticles), the flexible polymer chains can expulse larger particles at the surface, which is one of the thermodynamic mechanisms behind some of the self-healing[33], solar cells[34] or optically-advanced nano-composite materials[35]. This "depletion attraction" phenomenon allows for manipulation of particles to self-localize in specific regions of the material interfaces[33]. The time interval between each layer formation employed in the stereolithography process (i.e. 9 seconds) might not be long enough to allow for HA segregation at the interface between every layer. This could be the reason why HA enrichment was only observed at the surface of the SLA-fabricated scaffolds but not between every layer of the photo-crosslinked material.

Additionally, during the purification process, the SLA-fabricated scaffolds (initially soaked in propylene carbonate) undergo a solvent exchange using ethanol (as non-solvent) followed by a drying step. This solvent exchange is responsible for a swelling and then a deswelling of the materials, which could potentially trigger particles migration from the 
polymer network at the non-solvent/polymer interface, resulting in a corral-like spatial enrichment of the particles[17].

$\mathrm{CaP}$ mineral deposition is commonly performed to endow 3D-printed structures with osteogenic properties[14, 36, 37]. Nevertheless, the reported processes exhibit intrinsically several limitations, regarding for example the numerous post-printing steps required to create this mineral deposition. In addition, there is a limited physical integrity of the $\mathrm{CaP}$ deposited on the printed materials with risk of layer delamination and particle loosening. In this study, the HA enrichment observed on the SLA-fabricated PTMC/HA scaffolds did not require additional steps compared to the fabrication of PTMC only. Of importance, the HA particles are fully integrated in the polymer matrix (as shown figure 2 with the high amount of " $\mathrm{C}$ " element at the surface, originating from the PTMC matrix) conferring physical stability without risk of layer delamination and particle loosening.

Additionally, we observed different behaviour in terms of swelling of PTMC/HA which could only be partly attributed to the increased wettability of the structures containing high amount of HA (figure 3a). However, other parameters, such as $i$ - the density of crosslinking into the PTMC matrices which can be altered by the presence of HA, or $i i$ - the presence of HA in the bulk of the materials, can also influence the propensity of the photo-crosslinked scaffolds to swell, without having a direct impact on its wettability. For the protein adsorption study, we selected a medium with $10 \%$ of serum that naturally contains a mixture of proteins/growth factors as a model. We observed that PTMC 40 propensity to swell and also to adsorb proteins was significantly increased compared to the other groups. Those physico-chemical characteristics have a tremendous impact on bone healing, as several proteins have been shown to trigger cells recruitment, binding and differentiation[38], such as growth factors (e.g. BMPs, PDGF, TGF-b, etc.) but also platelet releasate and fibrinogen[25]. A surface that can bind more circulating proteins and formed quickly a fibrin clot will favour osteogenic cells (and platelets) to migrate through this transitory matrix, releasing in turn other cytokines and bone growth factors. By reaching the surface, bone cells will be able to synthetise de novo bone on the implant surface itself. This finding explains as well the significant enhancement of hBMSCs attachment witnessed on scaffolds containing $40 \mathrm{wt}$ \% HA (figure 4a).

Additionally, the increased bioactivity of the CaP loaded PTMC could be also attributed to the higher amount of $\mathrm{Ca}^{2+}$ released, figure $3 \mathrm{c}$. A major criterion of osteoconductive $\mathrm{CaP}$ based biomaterials relies in their ability to release free calcium in their microenvironment[21, 39]. Under normal condition, extracellular fluids are already supersaturated, but peri-prosthetic acidification happening during bone formation and remodelling (due to macrophages and osteoclasts activity) permits $\mathrm{Ca}^{2+}$ and phosphate ions dissolution and leads to the precipitation of carbonate hydroxyapatite layer (CHA) at the surface of the implant[38]. Additionally, $\mathrm{Ca}^{2+}$ released influences also the fate of osteoprogenitor cells in terms of adhesion, proliferation and differentiation[40, 41], which finally triggers the biomineralization cascade. 
In this study, we purposely selected un-sintered HA (containing more soluble calcium phosphate ions and an increased surface area), as sintered HA has been reported to resorb too slowly, which can provoke long-term complications[41]. Secondly, the surfaceenrichment of HA particles observed in PTMC 20 and PTMC 40 may facilitate $\mathrm{Ca}^{2+}$ dissolution and diffusion compared to homologues where $\mathrm{CaP}$ would be physically entrapped in crosslinked hydrophobic polymer matrices.

Several investigations have shown that the amount of HA exposed at the surface of composite scaffolds is of critical importance for neo-bone formation. As instance, Zhao et al. developed composite scaffolds (bioactive glass with polymers poly(3-hydroxybutyrateco-3-hydroxyhexanoate) (PHBHHx)) using 3D Bioplotter system. In vivo experiments revealed that more bone was formed in Bioglass/polymer composites, but that even in the group containing high amount of Bioglass (polymer/bioactive glass ratio of 1/7 w/w), the early bone deposition (at 3 weeks) was not in direct contact with the material surface, but was following a distance osteogenesis mechanism, similarly to the Bioglass-free scaffolds. They mentioned that the evaporation step included in the fabrication process was responsible to the creation of a thin polymeric layer covering the surface and the bioactive particles of the composite scaffold, which can have a direct impact on bone formation[42]. Similar new bone formation kinetic "extending from the edges of the defects towards the center of the defects" was mentioned by another recent work from Zeng et al. using PTMC doped with TCP ( $\beta$-tricalcium phosphate) or BCP (biphasic calcium phosphate), produced by salt-leaching technique[43]. Finally, using similar needle-shaped HA nanoparticles, Barbieri et al. demonstrated that only PDLLA composite scaffold with $40 \%$ weight HA (made by salt-leaching) could trigger ectopic bone formation, due to the highest amount of HA exposed at the surface of the scaffolds (impacting $\mathrm{Ca}^{2+}$ release, surface topography, protein adsorption, etc.), compared to scaffolds containing 10 or $20 \%$ of $\mathrm{CaP}$ [21]. Those are few examples showing that exposing HA to the surface of 3D printed constructs could favour osseointegration of the biomaterials. Nevertheless, no report on similar selfcorralling HA composite scaffolds has been presented so far, which opens great avenues in the field of bioactive scaffolds. Additionally, the influence of the nanoparticle shape on this segregation behaviour would definitely deserve more investigation, as other parameters such as particles concentration and size have been shown to influence polymer/nanoparticle blends' behaviour[44].

Finally, one of the singularities of the PTMC matrix degradation in opposition to the hydrolytic degradation of the polyesters, is the surface erosion mechanism[11]. The longterm success of any biomaterial-based tissue engineering strategy depends greatly on an appropriate degradation kinetic. Frequent failure attempts have been reported due to unsuitable scaffold resorption rates[45]. In vitro investigations have already demonstrated that photo-crosslinked PTMC is erodible, but at a rate significantly reduced compared to non-crosslinked PTMC (e.g. in cholesterol esterase solution, \pm 2200 versus $\pm 20 \mu \mathrm{g} / \mathrm{cm}^{2} \mathrm{x}$ day for PTMC and for photo-crosslinked PTMC respectively[46]). In our 6 weeks in vivo study, no evident sign of biomaterial degradation was observed and longer-term 
implantation period is required to specifically investigate PTMC degradation. Alternative copolymers, based on reported poly(TMC-co-CL) [47] or poly(TMC-co-DLLA)[48] could help in accelerating the biodegradation of the construct.

\section{Conclusions}

Material-directed endosseous integration is commonly obtained by combining macroporous scaffolds with osteogenic growth factors or with stem cells. Alternatively, emerging trends in the field of bone repair have paid more attention to the hierarchical fabrication of macro/microstructural designs of composite ceramic-polymer matrices, allowing to render those biomaterials osteopromotive.

We report on the fabrication of a scaffold made of poly(trimethylene carbonate) doped with hydroxyapatite nanoparticles for bone tissue engineering using stereolithography. Of interest, the SLA-process leads to a surface-enrichment of the HA in the composite PTMC/HA scaffolds. In vitro and in vivo experiments revealed the importance of microscale-structured PTMC/HA scaffolds for bone tissue engineering and how phase enrichment can potentially be used advantageously.

As perspective, optimisation of the interactions between nanoparticles and polymers in composite structures in combination with the stereolithography process could permit to engineer hierarchically ordered biomaterials with decreased loading of bioactive cues (i.e. nanoscopic HA) that exhibit tailored architecture and functionalities.

More generally, the possibility to control multiple length scales and dimensions could have remarkable applications in the burgeoning field of additive manufacturing systems.

\section{Acknowledgements}

Authors would like to acknowledge NSFC-DG-RTD Joint Scheme (Project No. 51361130034) and the European Union's 7th Framework Program under grant agreement $\mathrm{n}^{\circ}$ NMP3-SL-2013-604517, Project RAPIDOS. Additionally, we are grateful to the members of the preclinical facility (Zeiter S., Kluge K., Eberli, U. and Schmidt T.) and to Nehrbass D. for technical and histological assistance. Furthermore the authors express their gratitude to Dr. Davide Barbieri and Mr. Rongquan Duan in Kuros BioSciences BV for supplying hydroxyapatite nanoparticles.

\section{References:}

1. Giannoudis, P.V., H. Dinopoulos, and E. Tsiridis, Bone substitutes: an update. Injury, 2005. 36 Suppl 3: p. S20-27.

2. Bose, S., S. Vahabzadeh, and A. Bandyopadhyay, Bone tissue engineering using $3 D$ printing. Materials Today, 2013. 16(12): p. 496-504.

3. Hull, C.W., Apparatus for production of three-dimensional objects by stereolithography. 1986. 
4. Schuller-Ravoo, S., E. Zant, J. Feijen, and D.W. Grijpma, Preparation of a designed poly(trimethylene carbonate) microvascular network by stereolithography. Advanced Healthcare Materials, 2014. 3(12): p. 2004-2011.

5. Chapter 3 of this thesis; Geven, M.A., D. Barbieri, H. Yuan, J.D. de Bruijn, and D.W. Grijpma, Preparation and mechanical properties of photo-crosslinked poly(trimethylene carbonate) and nano-hydroxyapatite composites. Clinical Hemorheology \& Microcirculation, 2015. 60(1): p. 3-11.

6. Chapter 4 of this thesis; Geven, M.A., V. Varjas, L. Kamer, X. Wang, J. Peng, D. Eglin, and D.W. Grijpma, Fabrication of patient specific composite orbital floor implants by stereolithography. Polymers for Advanced Technologies, 2015. 26(12): p. 1433-1438.

7. Fukushima, K., Poly(trimethylene carbonate)-based polymers engineered for biodegradable functional biomaterials. Biomaterials Science, 2015. 4(1): p. 9-24.

8. Gentile, P., V. Chiono, I. Carmagnola, and P.V. Hatton, An overview of poly(lactic-co-glycolic) acid (PLGA)-based biomaterials for bone tissue engineering. International Journal of Molecular Science, 2014. 15(3): p. 36403659.

9. Taylor, M.S., A.U. Daniels, K.P. Andriano, and J. Heller, Six bioabsorbable polymers: in vitro acute toxicity of accumulated degradation products. Journal of Applied Biomaterials, 1994. 5(2): p. 151-157.

10. Ignatius, A.A., O. Betz, P. Augat, and L.E. Claes, In vivo investigations on composites made of resorbable ceramics and poly(lactide) used as bone graft substitutes. Journal of Biomedical Materials Research, 2001. 58(6): p. 701-709.

11. Zhang, Z., R. Kuijer, S.K. Bulstra, D.W. Grijpma, and J. Feijen, The in vivo and in vitro degradation behavior of poly(trimethylene carbonate). Biomaterials, 2006. 27(9): p. 1741-1748.

12. Pêgo, A.P., A.A. Poot, D.W. Grijpma, and J. Feijen, In Vitro Degradation of Trimethylene Carbonate Based (Co)polymers. Macromolecular Bioscience, 2002. 2(9): p. 411-419.

13. Bat, E., Z. Zhang, J. Feijen, D.W. Grijpma, and A.A. Poot, Biodegradable elastomers for biomedical applications and regenerative medicine. Regenerative Medicine, 2014. 9(3): p. 385-398.

14. Dadsetan, M., T. Guda, M.B. Runge, D. Mijares, R.Z. LeGeros, J.P. LeGeros, D.T. Silliman, L. Lu, J.C. Wenke, P.R. Brown Baer, and M.J. Yaszemski, Effect of calcium phosphate coating and rhBMP-2 on bone regeneration in rabbit calvaria using poly(propylene fumarate) scaffolds. Acta Biomater, 2015. 18: p. 9-20.

15. Lee, J.W., K.S. Kang, S.H. Lee, J.Y. Kim, B.K. Lee, and D.W. Cho, Bone regeneration using a microstereolithography-produced customized poly(propylene fumarate)/diethyl fumarate photopolymer $3 D$ scaffold incorporating BMP-2 loaded PLGA microspheres. Biomaterials, 2011. 32(3): p. 744-752. 
16. Danoux, C.B., D. Barbieri, H. Yuan, J.D. de Bruijn, C.A. van Blitterswijk, and P. Habibovic, In vitro and in vivo bioactivity assessment of a polylactic acid/hydroxyapatite composite for bone regeneration. Biomatter, 2014. 4: p. e27664.

17. Balazs, A.C., T. Emrick, and T.P. Russell, Nanoparticle polymer composites: where two small worlds meet. Science, 2006. 314(5802): p. 1107-1110.

18. Renna, L.A., C.J. Boyle, T.S. Gehan, and D. Venkataraman, Polymer Nanoparticle Assemblies: A Versatile Route to Functional Mesostructures. Macromolecules, 2015. 48(18): p. 6353-6368.

19. Mackay, M.E., A. Tuteja, P.M. Duxbury, C.J. Hawker, B. Van Horn, Z. Guan, G. Chen, and R.S. Krishnan, General strategies for nanoparticle dispersion. Science, 2006. 311(5768): p. 1740-1743.

20. Jouault, N., D. Zhao, and S.K. Kumar, Role of casting solvent on nanoparticle dispersion in polymer nanocomposites. Macromolecules, 2014. 47(15): p. 52465255.

21. Barbieri, D., A.J. Renard, J.D. de Bruijn, and H. Yuan, Heterotopic bone formation by nano-apatite containing poly(D,L-lactide) composites. Eur Cell Mater, 2010. 19: p. 252-261.

22. Karageorgiou, V. and D. Kaplan, Porosity of $3 D$ biomaterial scaffolds and osteogenesis. Biomaterials, 2005. 26(27): p. 5474-5491.

23. Herrmann, M., J.J. Bara, C.M. Sprecher, U. Menzel, J.M. Jalowiec, R. Osinga, A. Scherberich, M. Alini, and S. Verrier, Pericyte plasticity - comparative investigation of the angiogenic and multilineage potential of pericytes from different human tissues. Europen Cells \& Materials, 2016. 31: p. 236-249.

24. Kuzyk, P.R. and E.H. Schemitsch, The basic science of peri-implant bone healing. Indian Journal of Orthopeadics, 2011. 45(2): p. 108-115.

25. Davies, J.E., Understanding peri-implant endosseous healing. Journal of Dental Education, 2003. 67(8): p. 932-949.

26. Davies, J.E., In vitro modeling of the bone/implant interface. The Anatomical Record, 1996. 245(2): p. 426-445.

27. Meyer, U., U. Joos, J. Mythili, T. Stamm, A. Hohoff, T. Fillies, U. Stratmann, and H.P. Wiesmann, Ultrastructural characterization of the implant/bone interface of immediately loaded dental implants. Biomaterials, 2004. 25(10): p. 1959-1967.

28. Ramazanoglu, M. and Y. Oshida, Osseointegration and Bioscience of Implant Surfaces - Current Concepts at Bone-Implant Interface in Implant Dentistry - A Rapidly Evolving Practice, P.I. Turkyilmaz, Editor. 2011, INTECH.

29. Yuan, H., H. Fernandes, P. Habibovic, J. de Boer, A.M. Barradas, A. de Ruiter, W.R. Walsh, C.A. van Blitterswijk, and J.D. de Bruijn, Osteoinductive ceramics as a synthetic alternative to autologous bone grafting. PNAS, 2010. 107(31): p. 13614-13619. 
30. Davies, J.E., Mechanisms of endosseous integration. International Journal of Prosthodontics, 1998. 11(5): p. 391-401.

31. Davies, J.E., Bone bonding at natural and biomaterial surfaces. Biomaterials, 2007. 28(34): p. 5058-5067.

32. Puleo, D.A. and A. Nanci, Understanding and controlling the bone-implant interface. Biomaterials, 1999. 20(23-24): p. 2311-2321.

33. Lee, J.Y., G.A. Buxton, and A.C. Balazs, Using nanoparticles to create selfhealing composites. The Journal of chemical physics, 2004. 121(11): p. 55315540 .

34. Coakley, K.M. and M.D. McGehee, Conjugated Polymer Photovoltaic Cells. Chemistry of Materials, 2004. 16(23): p. 4533-4542.

35. Lin, Y., A. Boker, J. He, K. Sill, H. Xiang, C. Abetz, X. Li, J. Wang, T. Emrick, S. Long, Q. Wang, A. Balazs, and T.P. Russell, Self-directed self-assembly of nanoparticle/copolymer mixtures. Nature, 2005. 434(7029): p. 55-59.

36. Lan, P.X., J.W. Lee, Y.J. Seol, and D.W. Cho, Development of 3D PPF/DEF scaffolds using micro-stereolithography and surface modification. Journal of Materials Science: Materials in Medicine, 2009. 20(1): p. 271-279.

37. Luangphakdy, V., E. Walker, K. Shinohara, H. Pan, T. Hefferan, T.W. Bauer, L. Stockdale, S. Saini, M. Dadsetan, M.B. Runge, A. Vasanji, L. Griffith, M. Yaszemski, and G.F. Muschler, Evaluation of osteoconductive scaffolds in the canine femoral multi-defect model. Tissue Engineering Part A, 2013. 19(5-6): p. 634-648.

38. LeGeros, R.Z., Calcium phosphate-based osteoinductive materials. Chemical Reviews, 2008. 108(11): p. 4742-4753.

39. Metsger, D.S., R.M. DePhilip, and T.G. Hayes, An autoradiographic study of calcium phosphate ceramic bone implants in turkeys. Clinical Orthopeadics Related Research, 1993(291): p. 283-294.

40. Ducheyne, P. and Q. Qiu, Bioactive ceramics: the effect of surface reactivity on bone formation and bone cell function. Biomaterials, 1999. 20(23-24): p. 22872303.

41. Bohner, M., L. Galea, and N. Doebelin, Calcium phosphate bone graft substitutes: Failures and hopes. Journal of the European Ceramic Society, 2012. 32(11): p. 2663-2671.

42. Zhao, S., M. Zhu, J. Zhang, Y. Zhang, Z. Liu, Y. Zhu, and C. Zhang, Three dimensionally printed mesoporous bioactive glass and poly(3-hydroxybutyrate-co3-hydroxyhexanoate) composite scaffolds for bone regeneration. Journal of Materials Chemistry B, 2014. 2(36): p. 6106-6118.

43. Zeng, N., A.C. van Leeuwen, D.W. Grijpma, R.R. Bos, and R. Kuijer, Poly(trimethylene carbonate)-based composite materials for reconstruction of critical-sized cranial bone defects in sheep. Journal of Craniomaxillofacial Surgery, 2016. 45(2): p. 338-346. 
44. McGarrity, E.S., A.L. Frischknecht, and M.E. Mackay, Phase behavior of polymer/nanoparticle blends near a substrate. Journal of Chemistry \& Physics, 2008. 128(15): p. 154904.

45. Ulery, B.D., L.S. Nair, and C.T. Laurencin, Biomedical Applications of Biodegradable Polymers. Journal of Polymer Science Part B - Polymer Physics, 2011. 49(12): p. 832-864.

46. Bat, E., T.G. van Kooten, J. Feijen, and D.W. Grijpma, Resorbable elastomeric networks prepared by photocrosslinking of high-molecular-weight poly(trimethylene carbonate) with photoinitiators and poly(trimethylene carbonate) macromers as crosslinking aids. Acta Biomater, 2011. 7(5): p. 19391948.

47. Grijpma, D.W., Q. Hou, and J. Feijen, Preparation of biodegradable networks by photo-crosslinking lactide, epsilon-caprolactone and trimethylene carbonatebased oligomers functionalized with fumaric acid monoethyl ester. Biomaterials, 2005. 26(16): p. 2795-2802.

48. Sharifi, S. and D.W. Grijpma, Resilient amorphous networks prepared by photocrosslinking high-molecular-weight D,L-lactide and trimethylene carbonate macromers: mechanical properties and shape-memory behavior. Macromolecular Bioscience, 2012. 12(10): p. 1423-1435.

49. Barry, J.J., A.V. Evseev, M.A. Markov, C.E. Upton, C.A. Scotchford, V.K. Popov, and S.M. Howdle, In vitro study of hydroxyapatite-based photocurable polymer composites prepared by laser stereolithography and supercritical fluid extraction. Acta Biomater, 2008. 4(6): p. 1603-1610.

50. Scalera, F.C., E. Corcione, F. Montagna, A. Sannino, and A. Maffezzoli, Development and characterization of UV curable epoxy/hydroxyapatite suspensions for stereolithography applied to bone tissueengineering. Ceramics International, 2014. 40: p. 15455-15462.

51. Cooke, M.N., J.P. Fisher, D. Dean, C. Rimnac, and A.G. Mikos, Use of stereolithography to manufacture critical-sized $3 D$ biodegradable scaffolds for bone ingrowth. Journal of Biomedical Materials Research Part B - Applied Biomaterials, 2003. 64(2): p. 65-69.

52. Lee, K.W., S. Wang, B.C. Fox, E.L. Ritman, M.J. Yaszemski, and L. Lu, Poly(propylene fumarate) bone tissue engineering scaffold fabrication using stereolithography: effects of resin formulations and laser parameters. Biomacromolecules, 2007. 8(4): p. 1077-1084.

53. Kim, K., D. Dean, J. Wallace, R. Breithaupt, A.G. Mikos, and J.P. Fisher, The influence of stereolithographic scaffold architecture and composition on osteogenic signal expression with rat bone marrow stromal cells. Biomaterials, 2011. 32(15): p. 3750-3763. 
54. Elomaa, L., A. Kokkari, T. Närhi, and J.V. Seppälä, Porous 3D modeled scaffolds of bioactive glass and photocrosslinkable poly(e-caprolactone) by stereolithography. Composites Science and Technology, 2013. 74: p. 99-106.

55. Tanodekaew, S., S. Channasanon, and P. Uppanan, Preparation and degradation study of photocurable oligolactide-HA composite: a potential resin for stereolithography application. Journal of Biomedical Materials Research Part B Applied Biomaterials, 2014. 102(3): p. 604-611.

56. Ronca, A., L. Ambrosio, and D.W. Grijpma, Design of porous three-dimensional PDLLA/nano-hap composite scaffolds using stereolithography. J Appl Biomater Funct Mater, 2012. 10(3): p. 249-258.

57. Ronca, A., L. Ambrosio, and D.W. Grijpma, Preparation of designed poly $(D, L-$ lactide)/nanosized hydroxyapatite composite structures by stereolithography. Acta Biomater, 2013. 9(4): p. 5989-5996. 


\section{Chapter 7 - Supplementary information}

\section{Supplementary tables}

Supplementary table 1. Overview of the SLA-fabricated scaffolds for bone tissue engineering.

\begin{tabular}{|c|c|c|c|}
\hline \multicolumn{2}{|c|}{ Nature of the polymer } & \multirow{2}{*}{$\begin{array}{l}\text { Addition of osteopromotive cues } \\
\text { (expressed in \% weight in the final engineered } \\
\text { scaffold) } \\
\text { Hydroxyapatite }\left(\mathrm{Hydroxypol}^{\mathrm{TM}} \text { ) at } 30 \mathrm{wt} \%{ }^{*}\right. \\
\underline{\text { In vitro: Post-SLA treatment with supercritical }} \\
\mathrm{CO}_{2} \text { benefited human osteoblasts proliferation }\end{array}$} & \multirow{2}{*}{$\begin{array}{l}\text { Ref. } \\
\text { [49] }\end{array}$} \\
\hline \multirow[b]{2}{*}{$\frac{0}{\frac{0}{\frac{\pi}{\pi}}}$} & Poly(acrylic acid) & & \\
\hline & $\begin{array}{l}\text { Poly }(\text { Epoxy }) \\
\left.\text { (Cyracure }^{\circledR}\right)\end{array}$ & $\begin{array}{l}\text { Hydroxyapatite at up to } 60 \mathrm{wt} \text { \%* } \\
\text { Physico-chemical characterization: The } \\
\text { polymeric resin was used only as temporary } \\
\text { support and burned-out during HA sintering } \\
\text { process }\end{array}$ & {$[50]$} \\
\hline \multirow{4}{*}{ 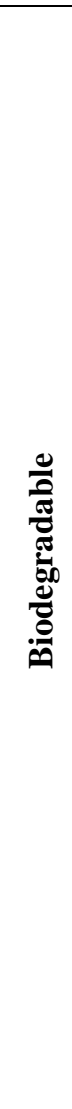 } & \multirow{4}{*}{$\begin{array}{l}\text { Poly(propylene } \\
\text { fumarate) }\end{array}$} & $\begin{array}{l}\text { No } \\
\text { Feasibility study: Investigation of the possibility } \\
\text { to build PPF-based structure using SLA }\end{array}$ & {$[51,52]$} \\
\hline & & $\begin{array}{l}\text { Coating of calcium-based minerals (either } \beta \text { - } \\
\text { tricalcium phosphate ( } \beta \text { TPC), carbonate } \\
\text { hydroxyapatite (synthetic bone mineral: SBM) or } \\
\text { biphasic calcium phosphate (BCP)) on the } \\
\text { surface of the scaffold post-SLA } \\
\text { In addition, rhBMP-2 was loaded on the surface } \\
\text { at three dosages ( } 0,50 \text { and } 100 \mu \mathrm{g} / \text { scaffold) } \\
\text { Critical-size calvarial model: Bone ingrowth was } \\
\text { relatively limited in calcium-based SLA } \\
\text { scaffolds, and only by combining with rhBMP-2 } \\
\text { an enhancement of the bone healing effect was } \\
\text { noted }\end{array}$ & [14] \\
\hline & & $\begin{array}{l}\text { No } \\
\text { In vitro: micro-architecture of SLA-scaffolds } \\
\text { influenced osteogenic response on rat BMSCs }\end{array}$ & {$[53]$} \\
\hline & & $\begin{array}{l}\text { Immersion of the scaffold in concentred } \\
\text { inorganic ions solution to form an apatite coating } \\
\text { layer combined with RGD peptide } \\
\text { In vitro: growth kinetic of pre-osteoblast on } \\
\text { coated scaffolds was improved compared to non- } \\
\text { coated controls. }\end{array}$ & [36] \\
\hline
\end{tabular}


Supplementary table 1. Continued.

\begin{tabular}{|c|c|c|c|}
\hline Nat & re of the polymer & $\begin{array}{l}\text { Addition of osteopromotive cues } \\
\text { (expressed in \% weight in the final engineered } \\
\text { scaffold) }\end{array}$ & Ref. \\
\hline & Poly(propylene & $\begin{array}{l}\text { BMP-2 loaded-PLGA microspheres }(0.05 \% \text { wt. } \\
\text { of BMP-2)* } \\
\text { Calvarial defect model: BMP-2 loaded scaffold } \\
\text { significantly increased bone formation compared } \\
\text { to non-loaded group ( } 80 \% \text { compared to } 25 \% \text { of } \\
\text { the area respectively covered with new bone after } \\
11 \text { weeks) }\end{array}$ & {$[15]$} \\
\hline$\cong$ & & $\begin{array}{l}\text { Coating of the scaffold (spray coating or dip } \\
\text { coating) with a dispersion of either } \\
\text { hydroxyapatite or } \beta \text { TPC on PLGA solution } \\
\text { Canine Femoral Multi-Defect Model: presence of } \\
\text { the mineral coating permitted new bone ingrowth } \\
\text { within } 4 \text { weeks (but absence of negative controls) }\end{array}$ & [37] \\
\hline 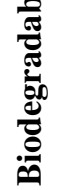 & $\begin{array}{l}\text { Poly }(\varepsilon- \\
\text { caprolactone })\end{array}$ & $\begin{array}{l}\text { Bioactive Glass (S53P4) at up to } 20 \mathrm{wt} . \% * \\
\text { In vitro: increasing BioGlass incorporation into } \\
\text { the PCL matrix enhanced fibroblast cells } \\
\text { proliferation }\end{array}$ & {$[54]$} \\
\hline & Poly(D,L-lactide) & $\begin{array}{l}\text { Hydroxyapatite at up to } 60 \mathrm{wt} . \% * \\
\text { In vitro: direct contact experiments performed on } \\
\text { 2D structures (using preosteoblasts) revealed a } \\
\text { satisfying cytocompatibility over a period of } 12 \\
\text { weeks }\end{array}$ & {$[55]$} \\
\hline & & $\begin{array}{l}\text { Nano-Hydroxyapatite at up to } 20 \mathrm{wt} . \% * \\
\text { In vitro: hBMSCs ALP activity is increased if } \\
\text { cultivated on SLA-scaffolds containing HA }\end{array}$ & {$[56,57]$} \\
\hline & $\begin{array}{l}\text { Poly(trimethylene } \\
\text { carbonate) }\end{array}$ & $\begin{array}{l}\text { Nano-Hydroxyapatite at up to } 40 \text { wt. } \% * \\
\text { Feasibility study: Investigation of the possibility } \\
\text { to build PTMC/HA-based non-porous composites } \\
\text { using SLA }\end{array}$ & {$[5,6]$} \\
\hline
\end{tabular}

Nature of the main composite biomaterials used for bone-tissue engineered scaffolds made by stereolithography, with a description of the main reported observation (* for those formulations, the bioactive compound was dispersed in the resin formulation before SLA processing). 
Surface-enrichment with nHA particles in composite scaffolds promotes bone repair

Supplementary table 2. Presentation of the resin formulations used to fabricate SLA-porous scaffolds by UV crosslinking technique.

\begin{tabular}{lccc}
\hline & \multicolumn{3}{c}{ Composition of the resins } \\
\cline { 2 - 4 } & PTMC & PTMC 20 & PTMC 40 \\
\hline PTMC-MA (wt. \%) & 60 & 36.4 & 25.1 \\
HA (wt. \%) & 0 & 9.1 & 16.7 \\
Propylene carbonate (wt. \%) & 40.0 & 54.5 & 58.2 \\
Lucirin $^{\circledR}$ TPO-L photo-initiator (wt. \%*) & 5 & 5 & 5 \\
Orasol Orange G dye (wt. \%*) & 0.15 & 0.10 & 0.08 \\
Intended amount of HA (wt. \%) & 0 & 20 & 40 \\
Experimental amount of HA (wt. $\%^{\$}$ ) & 0.5 & 23.8 & 46.5 \\
\hline
\end{tabular}

* Relative to PTMC-MA, ${ }^{\$}$ determined using Thermogravimetric analysis (TGA) on dried scaffolds 


\section{Supplementary figures}

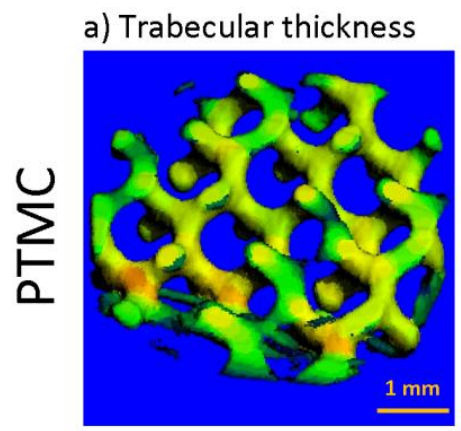

b) Trabecular space

c) Density mapping
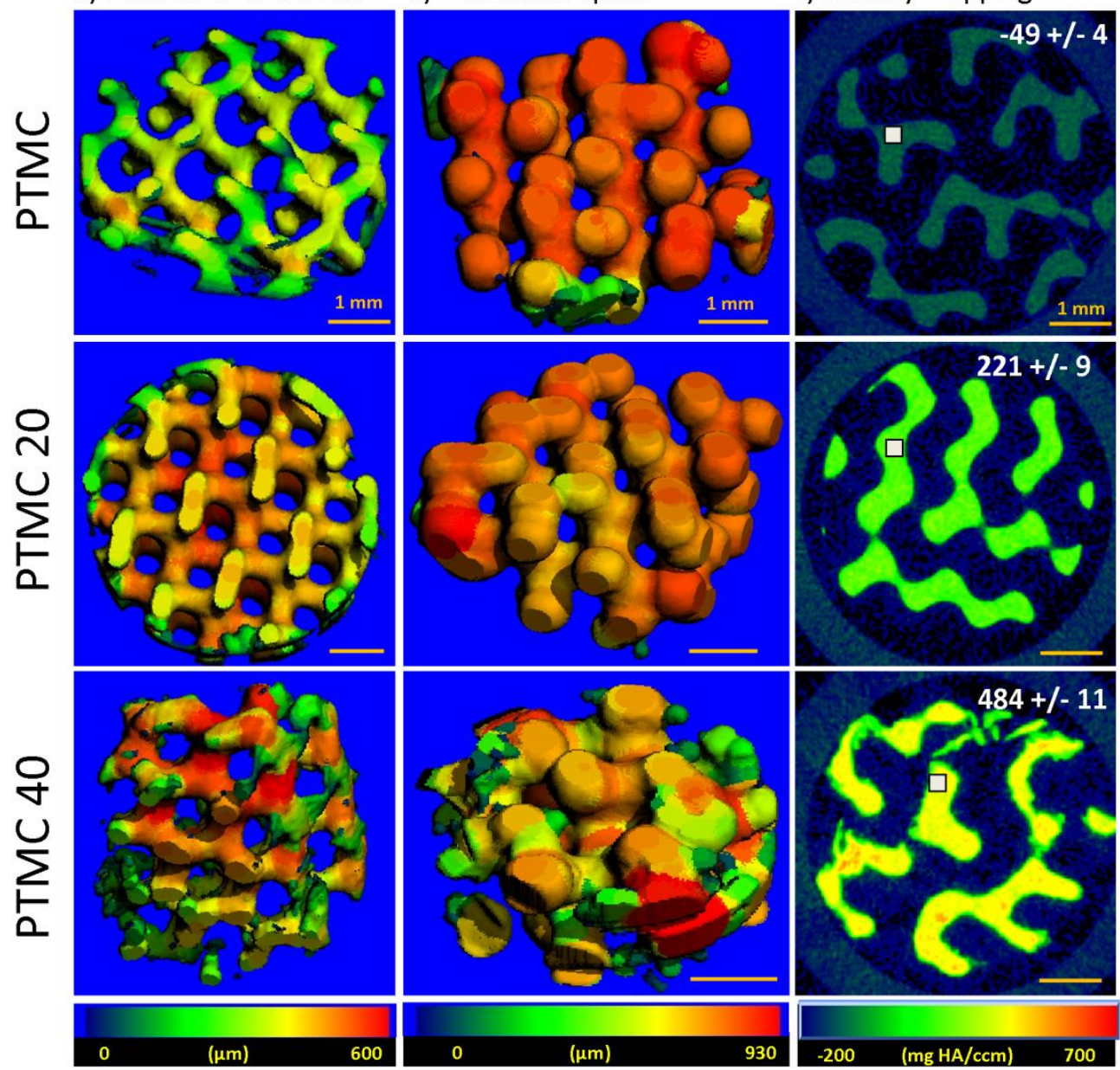

Supplementary figure 1. Architectural characterization of the composite PTMC scaffolds using microtomography. Cartographies of the distribution of a) the trabecular thickness (in $\mu \mathrm{m}$ ), of $b$ ) the trabecular space (in $\mu \mathrm{m}$ ) and of c) the material density (with scale expressed in $\mathrm{mg}$ of $\mathrm{HA} / \mathrm{ccm}$ ). 
a)

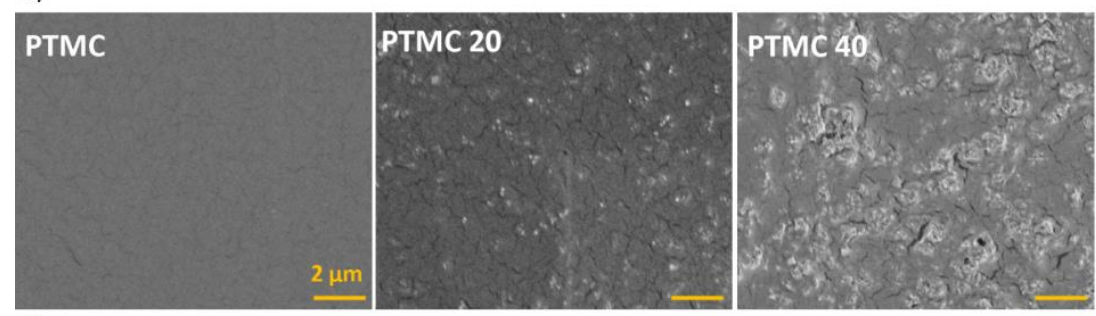

b)

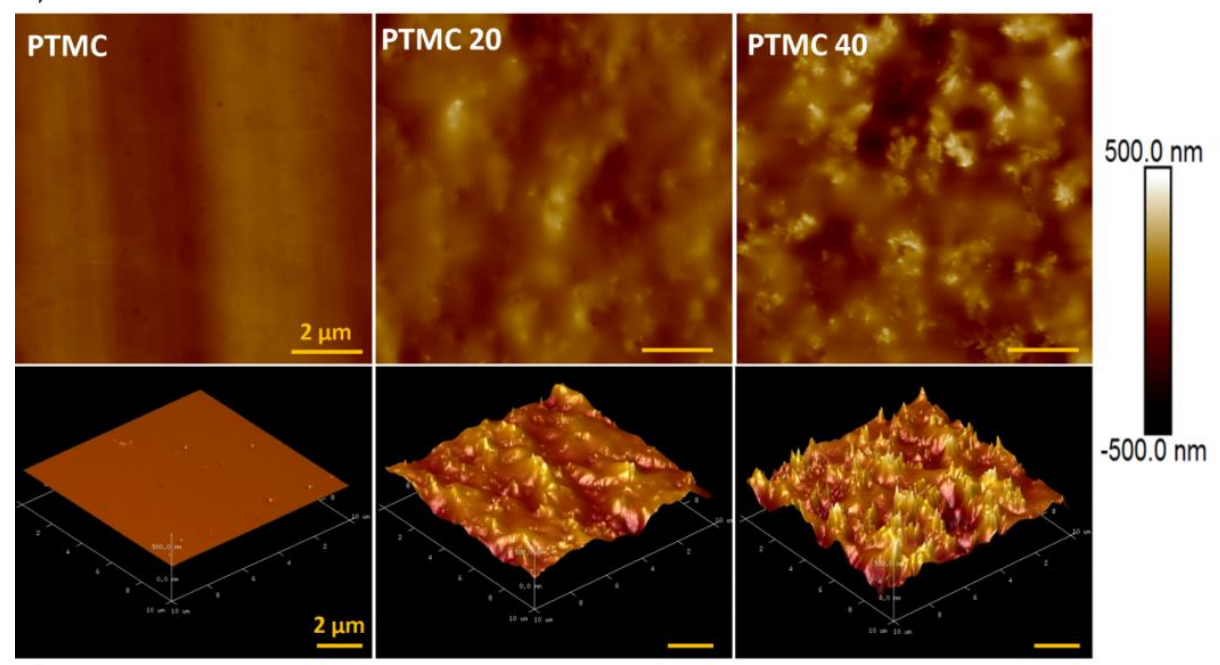

c)

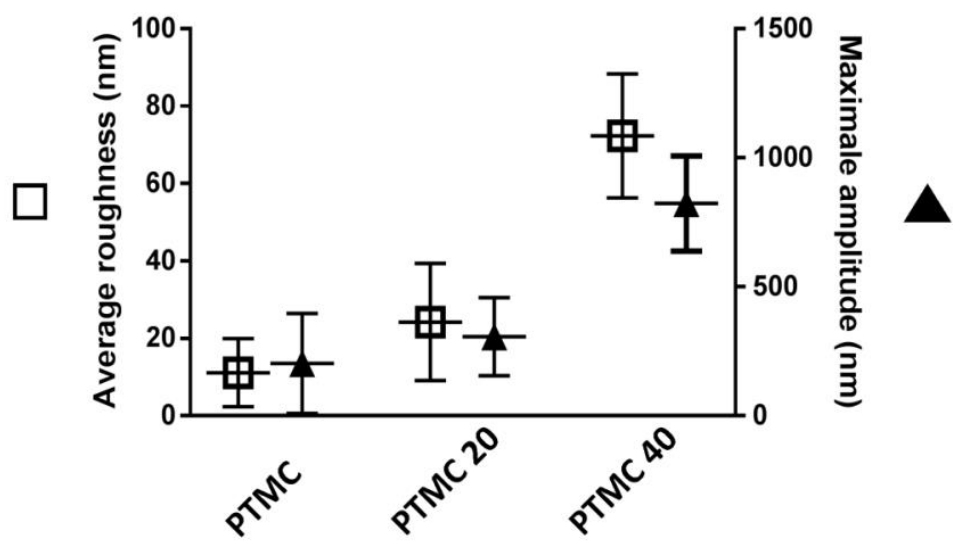

Supplementary figure 2. Topographical characterization of composite PTMC surfaces. a) SEM observation and b) atomic force microscopy (AFM) illustration and c) quantification of PTMC surface roughness influenced by HA incorporation. Material and Methods: SEM was conducted as mentioned Section 2.3.1. AFM analyses were performed on a Bruker Bioscope Catalyst system equipped with ScanAsyst (from Veeco) on 3 different surfaces of $10 \times 10 \mu \mathrm{m}^{2}$ at a scan rate of $0.996 \mathrm{~Hz}$. 


\section{Chapter 7}

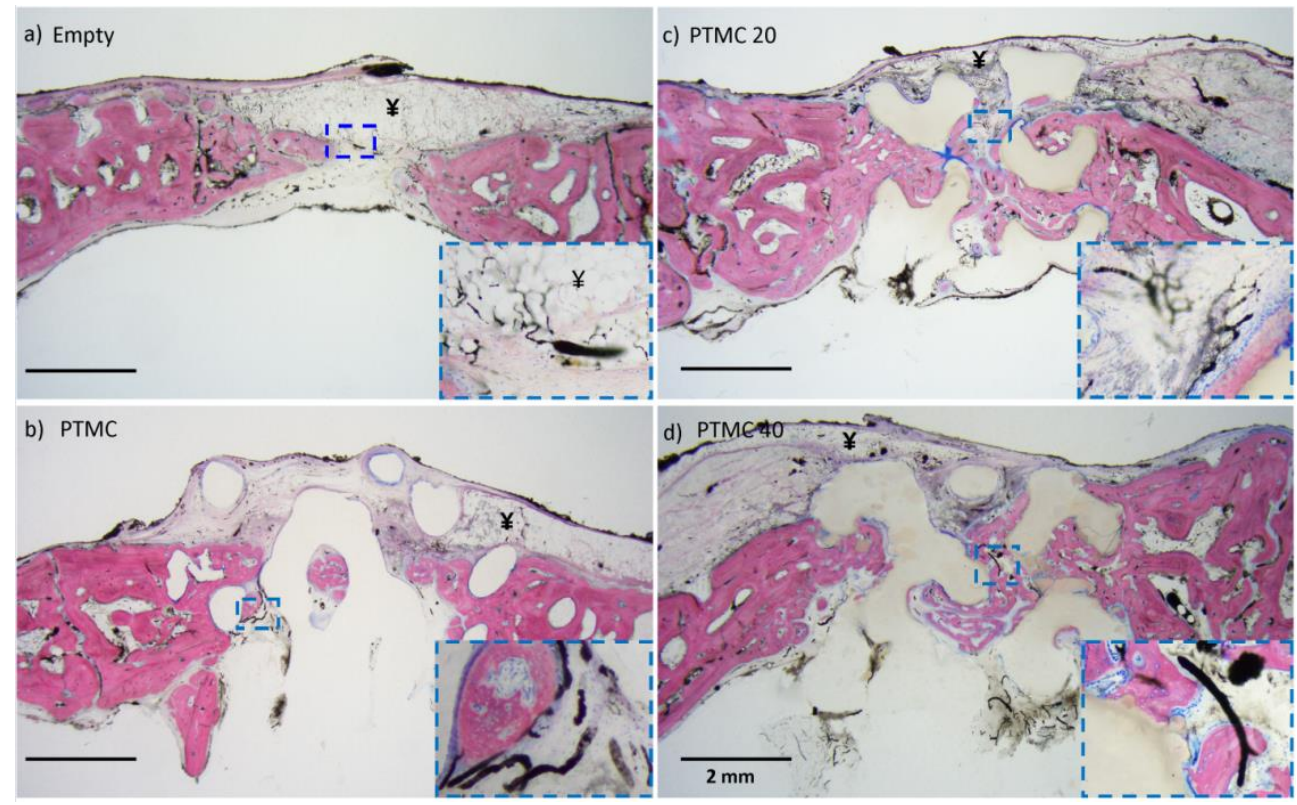

Supplementary figure 3. Histological observation of the successful revascularization of the implanted scaffolds. Histologic cross-sections following systemic perfusion of the euthanized animals using IndiaInk, staining in black the tissue vasculature occurring in the a) empty defect (mainly taking place in adipose tissue), or in the b) PTMC, c) PTMC 20 and d) PTMC 40 porous scaffolds. $¥$ denoted adipose tissue located either into the calvarial defect (for empty group) or subcutaneously (for PTMC and PTMC HA groups). Material and Methods: Vascularization of the defects was monitored by a post-mortal perfusion of IndiaInk (approximately $300 \mathrm{~mL} / \mathrm{animal}, n=4$ ). Euthanasia of the animals was realized after 6 weeks by IV injection of $600 \mathrm{mg}$ Pentobarbital. 


\title{
Chapter 8 - Micro-porous composite scaffolds of photo- crosslinked poly(trimethylene carbonate) and nano- hydroxyapatite prepared by low temperature extrusion- based additive manufacturing
}

Mike A. Geven ${ }^{\mathrm{a}}$, Christoph M. Sprecher ${ }^{\mathrm{b}}$, Olivier Guillaume ${ }^{\mathrm{b}}$, David Eglin ${ }^{\mathrm{b}}$ and Dirk W. Grijpma $^{\mathrm{a}}$

${ }^{a}$ Department of Biomaterials Science and Technology, University of Twente, Enschede, The Netherlands

${ }^{\mathrm{b}}$ AO Research Institute, Davos, Switzerland

\begin{abstract}
Complex bony defects such as those of the orbital floor are challenging to repair. Additive manufacturing techniques open up possibilities for the fabrication of implants with a designed macro-porosity for the reconstruction of such defects. Apart from a designed macro-porosity for tissue ingrowth, a micro-porosity in the implant struts will be beneficial for nutrient diffusion, protein adsorption and drug loading and -release.

In this work we report on a low temperature extrusion-based additive manufacturing method for the preparation of composite photo-crosslinked structures of poly(trimethylene carbonate) with bone-forming nano-hydroxyapatite and noricaritin (derived from bone growth stimulating icariin). In this method we extrude a dispersion of nano-hydroxyapatite and noricaritin particles in a solution of photo-crosslinkable poly(trimethylene carbonate) in ethylene carbonate into defined three dimensional structures. The ethylene carbonate is subsequently crystallized and extracted after photo-crosslinking.

We show that this results in designed macro-porous structures with micro-pores in the struts. The dispersion used to fabricate these structures shows favorable properties for extrusion-based processing, such as a sharp crystallization response and shear thinning. The formed photo-crosslinked materials have a micro-porosity of up to $48 \%$ and the E modulus, ultimate tensile strength and toughness are in excess of $24 \mathrm{MPa}, 2.0 \mathrm{~N} / \mathrm{mm}^{2}$ and 113 $\mathrm{N} / \mathrm{mm}^{2}$ respectively. A sustained release of noricaritin from these materials was also achieved. The results show that the technique described here is promising for the fabrication of micro-porous photo-crosslinked composite structures of poly(trimethylene carbonate) with nano-hydroxyapatite and that these may be applied in the reconstruction of orbital floor defects.
\end{abstract}




\section{Introduction}

Fabrication of scaffolds for the restoration of bony defects by additive manufacturing has gained increased attention in the last decade. A key asset of this type of fabrication technology is the ability to prepare well-defined structures with intricate and interconnected internal macro- porosities. In contrast, this is not possible by conventional techniques for the preparation of macro-porous structures such as salt leaching or gas foaming[1-3]. Additionally, the use of additive manufacturing techniques allows for the fabrication of patient-specific implants derived from computed tomography (CT) data[4-6]. This is especially useful for treatment of complex defects such as defects of the orbital floor and walls that require an accurate fit of the scaffold[7-9].

The majority of reports on the preparation of scaffolds for the repair of bony defects are on fused deposition modelling (FDM) of aliphatic polyesters and composites thereof[10-16]. FDM is a straightforward technique in which strands of molten polymer material are extruded at pre-defined locations and in a layer-by-layer manner to form three dimensional structures. Aliphatic polyesters such as poly(lactic-co-glycolic acid), poly(D,L-lactic acid) and poly( $\varepsilon$-caprolactone) or their mixtures are generally used since they can be meltprocessed and will crystallize after extrusion. Although encouraging biological results have been obtained using these polymers[15-17], polyesters are generally characterized by the formation of acidic compounds and deteriorating mechanical properties during their degradation[18, 19].

Linear, high molecular weight (HMW) poly(trimethylene carbonate) (PTMC) is a flexible amorphous polymer with a glass transition temperature of approximately $-20{ }^{\circ} \mathrm{C}$. It is a biodegradable polymer that degrades by an enzymatically driven surface erosion process. It has been previously determined that this process does not significantly decrease the bulk molecular weight of the polymer chains and that no acidic degradation products are formed[20, 21]. It is therefore a good candidate for the reconstruction of bone defects. However, since linear HMW PTMC is amorphous, it cannot easily be directly applied in additive manufacturing processes such as FDM. Lower molecular weight PTMC is semicrystalline, although it yields a rather waxy solid that crystallizes slowly from the melt and is not permanently form-stable[21, 22]. End-group functionalization of PTMC oligomers with methacrylate groups and subsequent photo-crosslinking has shown to be a good method for the preparation of form-stable PTMC structures [22].

Previously, we have reported on the fabrication of well-fitting implants for orbital floor defects by using three-armed, methacrylate end-group functionalized PTMC oligomers (PTMC-MA)[6]. Using stereolithography and a composite resin based on these macromers and bone-forming nano-hydroxyapatite (nHA) we were able to prepare fitting implants derived from patient CT reconstructions of fractured orbital floors. Although a macroporosity can readily be incorporated into the design of these implants, the struts will be dense. It is known however, that a micro-porosity in scaffolds for bone regeneration is beneficial for the supply of nutrients throughout the scaffold, for protein adsorption and for potential drug loading and release[23, 24]. Recently, Wang et al. showed it was possible to 
prepare micro-porous structures by stereolithography by using water-in-oil emulsions as resins[25]. In this case, scaffolds were produced with micro-pores of up to $50 \mu \mathrm{m}$ and up to 9 wt.\% hydroxyapatite.

Here we report on an alternative approach of preparing micro-porous, photo-crosslinked composite structures of PTMC-MA with 40 wt.\% of incorporated nHA. By extruding a dispersion of nHA in a solution of PTMC-MA in ethylene carbonate (EC) and subsequent crystallization of the solvent followed by photo-crosslinking and extraction, micro-porous structures could be prepared. EC is a good solvent for PTMC-MA, and has a melting point of $36{ }^{\circ} \mathrm{C}$. The obtained micro-porosity may have a positive effect on the reconstructive capabilities of photo-crosslinked PTMC-MA and nHA composite materials. We furthermore show that it allows for incorporation and release of noricaritin, a derivative of osteopromotive icariin which is applied in formulations for the treatment of viral infections and hyperplasia[26, 27].

\section{Experimental}

\subsection{Materials}

Trimethylene carbonate (TMC) was provided by Hiuzhou Foryou Medical Devices Co. Ltd.. Tin(II) ethylhexanoate $\left(\mathrm{Sn}(\mathrm{Oct})_{2}\right)$ was acquired from Spectrum Chemicals MFG. Corp.. Hydroquinone, triethylamine, methacrylic anhydride, ethylene carbonate, 1,1,1tris(hydroxymethyl)propane (TMP), phosphate buffered saline (PBS) tablets (for $0.01 \mathrm{M}$ phosphate buffer, $0.0027 \mathrm{M}$ potassium chloride and $0.137 \mathrm{M}$ sodium chloride, $\mathrm{pH}$ 7.4), Tween ${ }^{\circledR} 80$ and deuterated chloroform were from Sigma-Aldrich. Calcium hydride was obtained from Merck Millipore. Omnirad TPO-L photo-initiator was received from IMG Resins BV and Orasol Orange G from Ciba Specialty Chemicals. Kuros BioSciences BV kindly provided nHA in the form of a powder with aggregates $(\varnothing=15 \mu \mathrm{m})$ of needle-like hydroxyapatite crystals of 200 to $400 \mathrm{~nm}$ long and 20 to $50 \mathrm{~nm}$ wide. Noricaritin, a particulate solid, was acquired from Shanghai Yuanye Bio-Technology Co. Ltd. and sieved to a size of $20 \mu \mathrm{m}$ or smaller. Ethanol was acquired from Atlas \& Assink Chemie BV. Dichloromethane, methanol and chloroform were from VWR Chemicals. Dichloromethane was dried over calcium hydride and subsequently distilled under a dry $\mathrm{N}_{2}$ atmosphere. Materials were used as received unless stated otherwise.

\subsection{Synthesis of PTMC-MA and ink formulation}

A three-armed PTMC oligomer was prepared by ring-opening polymerization of TMC initiated by TMP. As reported previously[6, 28], TMC and TMP were charged in a flask under $\mathrm{N}_{2}$ atmosphere at a molar ratio of 96.7 to 1 (TMC/TMP). Under $\mathrm{N}_{2}$ the polymerization was conducted at $130{ }^{\circ} \mathrm{C}$ for 3 days using $\mathrm{Sn}(\mathrm{Oct})_{2}$ as a catalyst at a concentration of $0.13 \mathrm{wt} . \%$. Conversion of TMC was $98.5 \%$ and the number averaged molecular weight was $10.9 \mathrm{~kg} / \mathrm{mol}$ as was determined by ${ }^{1} \mathrm{H}-\mathrm{NMR}$ as previously reported[28] (Bruker Ascend 400/Avance III $400 \mathrm{MHz}$ NMR spectrometer, performed in deuterated chloroform). 
The prepared PTMC oligomer was functionalized with methacrylic anhydride to obtain PTMC-MA. As previously reported, the PTMC oligomer was dissolved in dichloromethane under $\mathrm{N}_{2}$ atmosphere. While stirring, $0.1 \mathrm{wt} . \%$ hydroquinone, triethylamine $(9 \mathrm{~mol} / \mathrm{mol}$ PTMC oligomer) and methacrylic anhydride (9 mol/mol PTMC oligomer) were added to the reaction mixture. This was stirred in the dark for 4 days, after which it was precipitated in ice-cooled methanol. The precipitate was thereafter extracted in fresh, ice-cooled methanol to additionally purify it. Subsequently it was dried in vacuo in the dark over 7 days. The degree of functionalization was higher than $99 \%$ as determined by ${ }^{1} \mathrm{H}-\mathrm{NMR}[28]$. Two different dispersions of nHA in PTMC-MA solutions in EC, hereafter referred to as 'inks', with and without noricaritin (PTMC-MA/nHA/N and PTMC-MA/nHA respectively) were prepared. For both inks nHA was first dispersed in chloroform and PTMC-MA was thereafter dissolved in this dispersion at $0.1 \mathrm{~g} / \mathrm{ml}$ by stirring overnight. The mass ratio of nHA to PTMC-MA was 0.67 to 1 in order to fabricate structures with 40 wt. $\%$ nHA. Subsequently the dispersion was precipitated in ice-cooled methanol and the precipitate was dried in vacuo in the dark for 3 days. The dried precipitate was mixed with $\mathrm{EC}$ at $50{ }^{\circ} \mathrm{C}$ for a day after which it was sonicated for 30 minutes. Omnirad TPO-L was thereafter added at $50{ }^{\circ} \mathrm{C}$. In PTMC-MA/nHA/N, noricaritin was subsequently mixed in as well. Table 1 provides an overview of the composition of the prepared inks.

Table 1. Formulation of inks used in this work. Values are expressed in wt.\%.

\begin{tabular}{lcc}
\hline & \multicolumn{2}{c}{ Inks } \\
\cline { 2 - 3 } Component & PTMC-MA/nHA & PTMC-MA/nHA/N \\
\hline PTMC-MA & 28.0 & 27.9 \\
nHA & 18.6 & 18.6 \\
Ethylene carbonate & 52.0 & 51.9 \\
Omnirad TPO-L & 1.4 & 1.4 \\
Noricaritin & 0.0 & 0.2 \\
\hline
\end{tabular}

\subsection{Preparation of micro-porous photo-crosslinked films and extrusion-based additive manufacturing}

For low temperature extrusion-based additive manufacturing (LTEAM) experiments, a RegenHU 3D Discovery apparatus was used. PTMC-MA/nHA ink was transferred to $3 \mathrm{ml}$ Nordson EFD black syringe barrels. These were equipped with Nordson EFD precision stainless steel extrusion tips with an internal diameter of $0.33 \mathrm{~mm}$ and RegenHU flat-wall pistons. The syringe barrels were installed in a direct dispenser cartridge heater of the RegenHU 3D Discovery apparatus. During extrusion a pressure of 2 bar and a temperature of $55{ }^{\circ} \mathrm{C}$ was applied. The rate of the movement of the cartridge and extrusion tip was set to $6 \mathrm{~mm} / \mathrm{s}$. The step height in the Z-direction between every layer was set to $0.26 \mathrm{~mm}$. Material was extruded within the space of a $1 \mathrm{~cm}$ high cooled aluminium ring placed on an aluminium block at $7{ }^{\circ} \mathrm{C}$. Structures were extruded using the patterns illustrated in figure 1, subsequently extruding 3 times layer $\mathrm{A}$ and subsequently three times layer B until 10 layers 
were deposited (AAABBBAAAB). Directly after extrusion the structures were transferred to a UV crosslinking box where they were photo-crosslinked for 30 minutes at $365 \mathrm{~nm}, 11$ $\mathrm{mW} / \mathrm{cm}^{2}$ and at room temperature. Thereafter the structures were extracted in demineralized water for 24 hours, refreshing the extraction medium 3 times. The structures were subsequently dried under ambient conditions for 7 days.

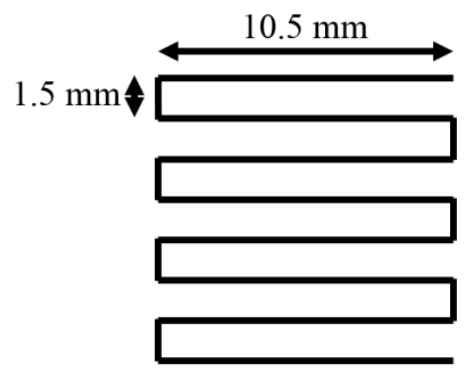

Layer A

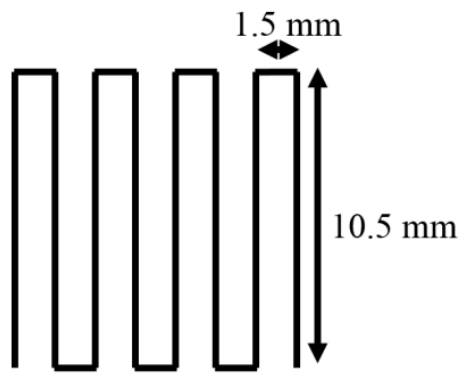

Layer B

Figure 1. Illustration of patterns extruded in LTEAM for fabrication of photo-crosslinked PTMC-MA/nHA microporous composites.

For the characterization of mechanical properties and noricaritin release, thin micro-porous films were prepared in analogy to LTEAM. Both inks were solvent cast at $0.5 \mathrm{~mm}$ thickness on glass plates at $55^{\circ} \mathrm{C}$ and thereafter cooled to $7{ }^{\circ} \mathrm{C}$. Subsequently they were photo-crosslinked, extracted and dried as described above, which yielded films of 0.24 to $0.34 \mathrm{~mm}$ thick.

\subsection{Characterization of inks}

The thermal properties of the inks were characterized by differential scanning calorimetry (DSC) on a Perkin Elmer Pyris 1 . Under $\mathrm{N}_{2}$, samples $(\mathrm{n}=3)$ were heated from -20 to $60{ }^{\circ} \mathrm{C}$ at $5{ }^{\circ} \mathrm{C} /$ minute. The samples were subsequently kept at $60{ }^{\circ} \mathrm{C}$ for 1 minute after which they were cooled to $-20{ }^{\circ} \mathrm{C}$ at $5{ }^{\circ} \mathrm{C} /$ minute. Subsequently the samples were kept at $-20{ }^{\circ} \mathrm{C}$ for 1 minute after which an identical heating and cooling cycle was performed. Melting points of the inks were determined from the peak temperatures in the first heating cycle, whereas the crystallization temperatures were determined from the peak temperatures from the second cooling cycle.

The viscosity of the inks was investigated by rheology. These experiments were performed in threefold on an Anton Paar Physica MCR-201 equipped with a PP25 measurement probe with a plate diameter of $25 \mathrm{~mm}$. All samples were loaded at the center of the measurement probe at $55^{\circ} \mathrm{C}$ and excess sample was carefully removed after setting the measurement gap of $3 \mathrm{~mm}$. Viscosity was monitored at $55{ }^{\circ} \mathrm{C}$ after a pre-shear of $10 \mathrm{~s}^{-1}$ for 5 minutes and $0.01 \mathrm{~s}^{-1}$ for 2 minutes. Shear rate was thereafter increased from 0.01 to $100 \mathrm{~s}^{-1}$ with a slope of 30 measuring points per decade and 6 seconds between measuring points. 


\subsection{Characterization of micro-porous photo-crosslinked films, noricaritin release and fabricated scaffolds}

The porosity of the micro-porous photo-crosslinked films was determined gravimetrically on disk shaped samples with a diameter of $6 \mathrm{~mm}(\mathrm{n}=3)$ which were die-cut from the films. Equation 1 was used for this calculation.

$$
P=\left(1-\frac{m_{S}}{\left(f_{P T M C} \rho_{P T M C}+f_{n H A} \rho_{n H A}\right) V_{S}}\right) * 100 \%
$$

In equation $1, P$ is the porosity in $\%, \mathrm{~m}_{\mathrm{s}}$ is the measured mass of the sample in $\mathrm{g}, V_{s}$ is the volume of the sample in $\mathrm{cm}^{3}, f_{P T M C}$ and $\rho_{P T M C}$ are the mass fraction (0.6) and density (1.31 $\mathrm{g} / \mathrm{cm}^{3}$ ) of PTMC respectively. Furthermore, $f_{n H A}$ and $\rho_{\mathrm{nHA}}$ are the weight fraction $(0.4)$ and the theoretical density $\left(3.162 \mathrm{~g} / \mathrm{cm}^{3}\right)$ of nHA based on its crystal structure respectively.

The degree of swelling of the micro-porous photo-crosslinked films in chloroform was assessed using disk shaped samples with a diameter of $6 \mathrm{~mm}(\mathrm{n}=3)$ that were die-cut from the films. The samples were swollen in chloroform for 24 hours and their mass was determined before immersion $\left(\mathrm{m}_{\mathrm{i}}\right)$ and after immersion and dabbing with tissue paper $\left(\mathrm{m}_{\mathrm{c}}\right)$. The degree of swelling was calculated using equation 2.

$$
S=\frac{\left(m_{c}-m_{i}\right)}{V_{i} \rho_{c}} * 100 \%
$$

In equation 2, $S$ is the degree of swelling in $\%, V_{i}$ is the initial sample volume in $\mathrm{cm}^{3}$ and $\rho_{c}$ is the density of chloroform $\left(1.489 \mathrm{~g} / \mathrm{cm}^{3}\right)$.

The mechanical properties of micro-porous photo-crosslinked films were determined in tensile experiments conducted on a Zwick Z020 tensile tester equipped with a $500 \mathrm{~N}$ load cell. Strips $(\mathrm{n}=5)$ measuring $10 \times 0.5 \mathrm{~cm}^{2}$ were die-cut from the prepared films. An initial grip-to-grip separation of $5 \mathrm{~cm}$ and a crosshead speed of $5 \mathrm{~cm} /$ minute were used. The $\mathrm{E}$ modulus, ultimate tensile strength and elongation at break, yield strength and elongation at yield and toughness of the films was determined from engineering stress-strain curves derived from the tensile experiments.

Drug release experiments were performed on disk-shaped samples $(\mathrm{n}=3)$ with an $8 \mathrm{~mm}$ diameter that were die-cut from thin films. These were placed in $4.5 \mathrm{ml}$ of PBS supplemented with $0.5 \%$ (w/v) Tween ${ }^{\circledR} 80$ (PBST) and subsequently incubated at $37{ }^{\circ} \mathrm{C}$ while shaken on a rotary shaker at $100 \mathrm{rpm}$. At regular time intervals $4 \mathrm{ml}$ of incubated PBST was replaced by fresh PBST. Incubated PBST was analyzed by UV-Vis spectroscopy (Agilent Technologies Cary 300) to determine the concentration of noricaritin in the release medium.

Fabricated scaffolds were characterized by micro computed tomography $(\mu \mathrm{CT})$ and by high resolution scanning electron microscopy (HR-SEM). Scaffold visualization by $\mu \mathrm{CT}$ was performed on a Scanco Medical $\mu \mathrm{CT}$ 40. Three dimensional reconstructions of the 
scaffolds were prepared using a bone trabecular morphometry script with a lower threshold corresponding to $85 \mathrm{mg}$ hydroxyapatite $/ \mathrm{cm}^{3}$. Structures were analyzed using the local thickness method developed by Hildebrand et al.[29]. Cross-sections of freeze-fractured scaffolds were viewed in HR-SEM by a Zeiss Merlin scanning electron microscope equipped with high efficiency scanning electron detector at 2 to $5 \mathrm{kV}$. Micro-pore size was determined using three separate HR-SEM images of cross-sections of scaffold struts. The diameter of at least 100 randomly selected pores per image was analyzed in ImageJ.

\section{Results and discussion}

\subsection{Thermal behavior of inks}

Using DSC, the melting and crystallization of inks was investigated. Figure 2 shows representative DSC thermograms of both inks. PTMC-MA/nHA showed a broad melting transition with a peak at $35 \pm 0.2{ }^{\circ} \mathrm{C}$. This is close to the melting temperature of ethylene carbonate $\left(36{ }^{\circ} \mathrm{C}\right)$ as well as that of PTMC-MA of $10 \mathrm{~kg} / \mathrm{mol}\left(33^{\circ} \mathrm{C}\right)[22]$. The crystallization transition of PTMC-MA/nHA is rather sharp at $13 \pm 3.1{ }^{\circ} \mathrm{C}$. When adding noricaritin, the melting and crystallization temperatures shift to lower values of $32 \pm 0.4{ }^{\circ} \mathrm{C}$ and $7.0 \pm 2.8^{\circ} \mathrm{C}$, respectively. These results indicate that mild processing conditions can be applied during LTEAM since no severe heating is required during extrusion and no severe cooling to crystallize extruded structures. However, it should be noted that the subsequent photo-crosslinking reaction is exothermic and that cooling during this process should be sufficient to ensure form-stability.

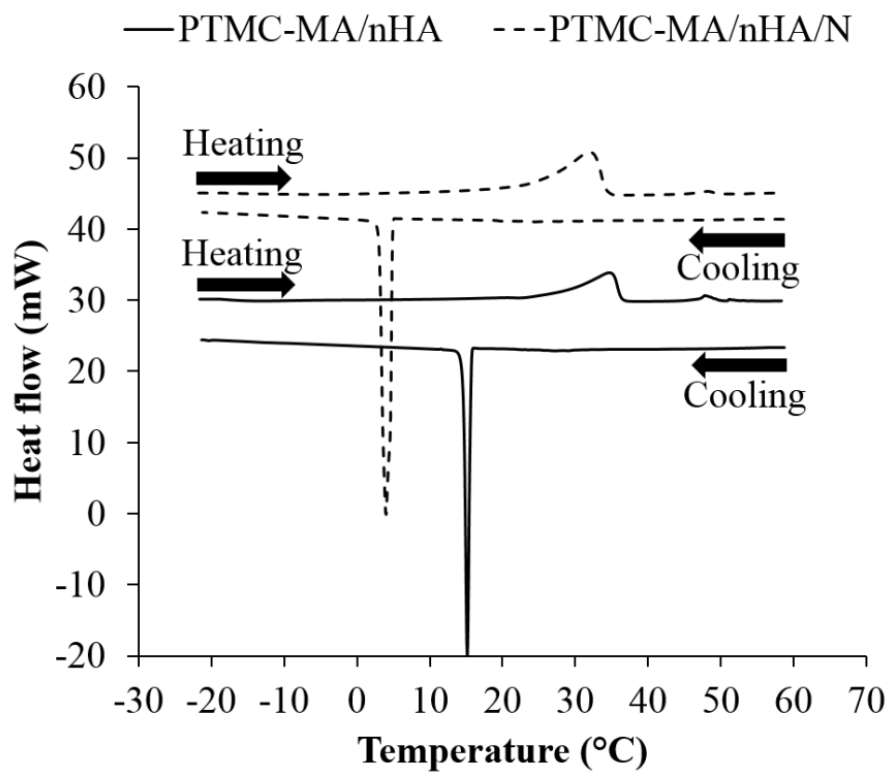

Figure 2. DSC thermograms of the PTMC-MA/nHA and PTMC-MA/nHA/N inks. 


\subsection{Shear behavior of inks}

Rheological experiments showed that the viscosity of the inks were strongly dependent on shear rate. Figure 3 shows representative examples of the viscosity dependence on shear rate of both inks at $55{ }^{\circ} \mathrm{C}$. Both inks are strongly shear thinning. The viscosity ranged from $103 \pm 28 \mathrm{~Pa} \cdot \mathrm{s}$ at $0.01 \mathrm{~s}^{-1}$ to $2.4 \pm 0.5 \mathrm{~Pa} \cdot \mathrm{s}$ at $100 \mathrm{~s}^{-1}$ for PTMC-MA/nHA. For PTMC$\mathrm{MA} / \mathrm{nHA} / \mathrm{N}$ the measured viscosity was higher, although the shear thinning effect was strong as well. The viscosity of this ink ranged between $3.5 \cdot 10^{5} \pm 1.3 \cdot 10^{5} \mathrm{~Pa} \cdot \mathrm{s}$ at $0.01 \mathrm{~s}^{-1}$ and $19 \pm 0.9 \mathrm{~Pa} \cdot \mathrm{s}$ at $100 \mathrm{~s}^{-1}$. This strong shear thinning may be beneficial for extrusion-based additive manufacturing as the lower viscosity under shear during extrusion allows for relatively low pressures to be applied. Once extruded, the increase in viscosity in the absence of shear may prevent excessive flowing of extruded material.

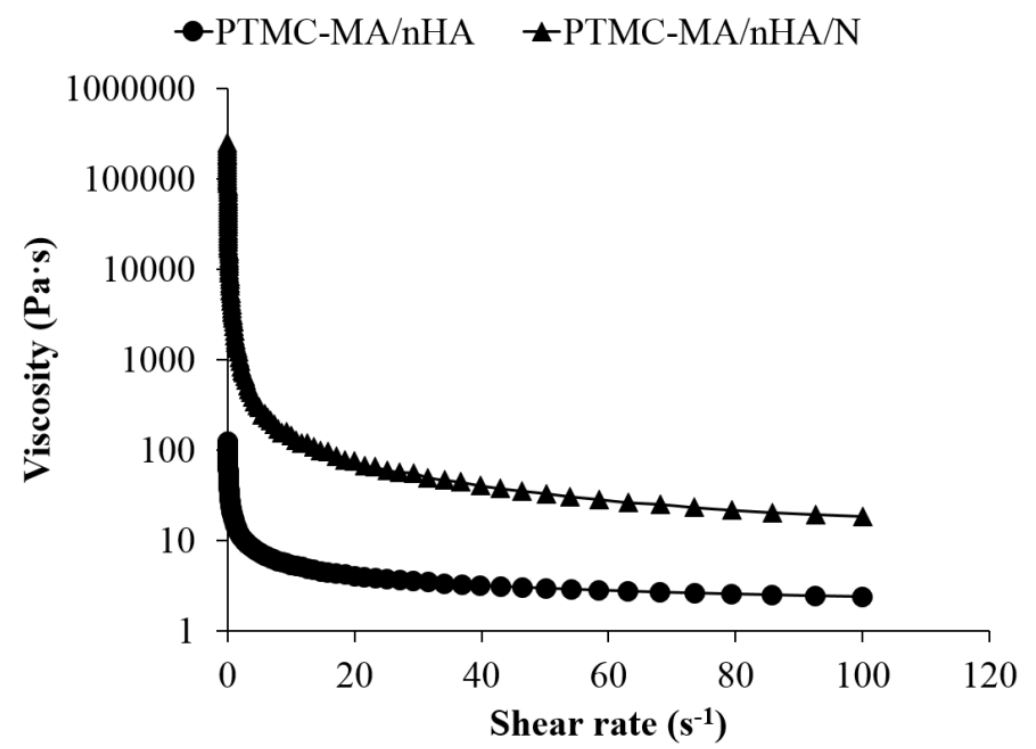

Figure 3. Viscosity as a function of shear rate of the PTMC-MA/nHA and PTMC-MA/nHA/N inks.

\subsection{Mechanical characterization of micro-porous photo-crosslinked films}

Using a gravimetric method, the porosity of the micro-porous photo-crosslinked films was determined. Films prepared using PTMC-MA/nHA had a porosity of $44 \pm 9.2 \%$. Films prepared using PTMC-MA/nHA/N had a porosity of $48 \pm 1.6 \%$. The degree of swelling of the films in chloroform was also determined gravimetrically. The degree of swelling of the photo-crosslinked PTMC-MA/nHA films was $314 \pm 15 \%$ and $291 \pm 23 \%$ of PTMC$\mathrm{MA} / \mathrm{nHA} / \mathrm{N}$ films. Thus, no significant difference between the porosity and the degree of swelling of both types of photo-crosslinked films was found.

To investigate the mechanical properties of micro-porous photo-crosslinked PTMC$\mathrm{MA} / \mathrm{nHA}$ and PTMC-MA/nHA/N composites, thin films were prepared and subjected to 
tensile testing. In table 2 an overview is given of the mechanical properties determined from these experiments together with the values found for the porosity and the degrees of swelling of the films. Incorporation of noricaritin in the photo-crosslinked composites lowers the E modulus, yield strength and ultimate tensile strength compared to the composites without noricaritin. Nonetheless, the toughness of both materials was similar. The E modulus of the photo-crosslinked PTMC-MA/nHA/N was $24 \mathrm{MPa}$, with a yield strength of $0.5 \mathrm{MPa}$. In applications with low loading such as orbital floor reconstruction (where peak stresses on the implant are $4 \mathrm{kPa}[30]$ ) this should be sufficient.

Table 2. Characteristic properties of photo-crosslinked composite films prepared from PTMC-MA/nHA and PTMC-MA/nHA/N inks.

\begin{tabular}{lcc}
\hline & \multicolumn{2}{c}{ Inks } \\
\cline { 2 - 3 } Property & PTMC-MA/nHA & PTMC-MA/nHA/N \\
\hline Porosity $(\%)$ & $44 \pm 9.2$ & $48 \pm 1.6$ \\
Degree of swelling (\%) & $291 \pm 23$ \\
E modulus $(\mathrm{MPa})$ & $314 \pm 15$ & $24 \pm 1.7$ \\
Yield strength $\left(\mathrm{N} / \mathrm{mm}^{2}\right)$ & $32 \pm 1.7$ & $0.5 \pm 0.03$ \\
Strain at yield $(\%)$ & $0.7 \pm 0.03$ & $2.8 \pm 0.1$ \\
Ultimate tensile strength $\left(\mathrm{N} / \mathrm{mm}^{2}\right)$ & $2.7 \pm 0.1$ & $2.0 \pm 0.2$ \\
Strain at break $(\%)$ & $2.4 \pm 0.2$ & $78 \pm 18$ \\
Toughness $\left(\mathrm{N} / \mathrm{mm}^{2}\right)$ & $73 \pm 11$ & $113 \pm 35$ \\
\hline
\end{tabular}

\subsection{Noricaritin release from micro-porous photo-crosslinked films}

The cumulative release of noricaritin was determined by incubation of photo-crosslinked PTMC-MA/nHA/N samples in PBST and determination of the noricaritin concentration by $\mathrm{UV}-\mathrm{V}$ is spectroscopy. The cumulative release profile is shown in figure 4 . The noricaritin release from photo-crosslinked PTMC-MA/nHA/N is characterized by a relatively rapid release in the first five days. Thereafter a sustained noricaritin release was observed for 30 days.

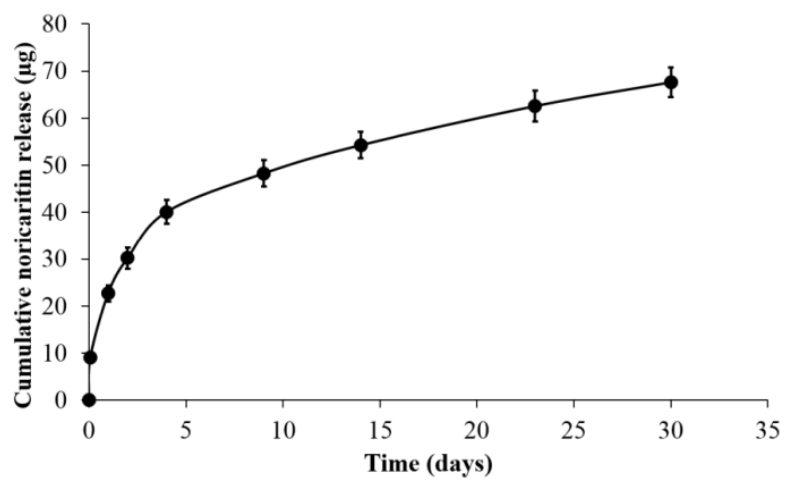

Figure 4. Cumulative noricaritin release from the photo-crosslinked PTMC-MA/nHA/N film. 


\subsection{Fabricated scaffolds}

Scaffolds were fabricated by LTEAM by extrusion of the PTMC-MA/nHA ink at $55^{\circ} \mathrm{C}$ onto a build plate at $7{ }^{\circ} \mathrm{C}$. The extrusion temperature setting was relatively high compared to the melting temperature of the inks due to the extrusion setup used. In this setup only the syringe barrel was heated but not the extrusion tip at its extremity. Even lower extrusion temperatures may be applied in a setup in which the temperature of the tip is also controlled.

Scaffolds fabricated by LTEAM are depicted in figure 5, together with $\mu$ CT reconstructions and HR-SEM images of cross-sections of the scaffolds. Scaffolds were prepared with a spacing of $1.5 \mathrm{~mm}$ between the extruded struts. Using $\mu \mathrm{CT}$ (figure $5 \mathrm{C}$ and D), it was determined that the average spacing between the struts in the photo-crosslinked scaffolds was $0.98 \pm 0.11 \mathrm{~mm}$ with an average strut width of $0.48 \pm 0.06 \mathrm{~mm}$. The average height of the printed layers was $0.36 \pm 0.09 \mathrm{~mm}$. The width of the struts and the height of the printed layers are larger than the diameter of the extrusion tip. This is likely the result of die-swell upon exiting of the ink from the tip and sagging upon deposition of the fibers. Nonetheless, $\mu \mathrm{CT}$ also indicated that both the vertical and horizontal pore channels were homogeneous in size and fully interconnected. Apart from the designed macro pore channels, small isolated pores were present in the structure. These defects likely arose from air bubbles entrapped in the extruded ink. This may be resolved by more rigorous degassing of the inkloaded syringe barrels before application in LTEAM.

In figure 5E a HR-SEM image of the cross-section of a fabricated structure is shown. Here the stacking of printed layers can be observed. In figure $5 \mathrm{~F}$ and $\mathrm{G}$, micro-pores could clearly be observed on the scaffold surface and in the struts of fabricated structures respectively. The internal diameter of these micro-pores was between 1 and $7 \mu \mathrm{m}$ and the average diameter was $3.5 \mu \mathrm{m}$. The orientation of the pores, i.e. EC crystal growth direction, was heterogeneous throughout the scaffold. The presence of these micro-pores indicates that fabricated structures remain sufficiently crystallized during the photo-crosslinking process even though heat is evolved and no active cooling is applied.

At higher magnifications, the polymer matrix in-between micro-pores could be viewed in detail (figure $5 \mathrm{H}$ ). In the image, white needle-like nHA particles were clearly seen and were well separated within the photo-crosslinked PTMC-MA matrix. This shows that a homogeneous dispersion was achieved and subsequently maintained during the extrusion process. 


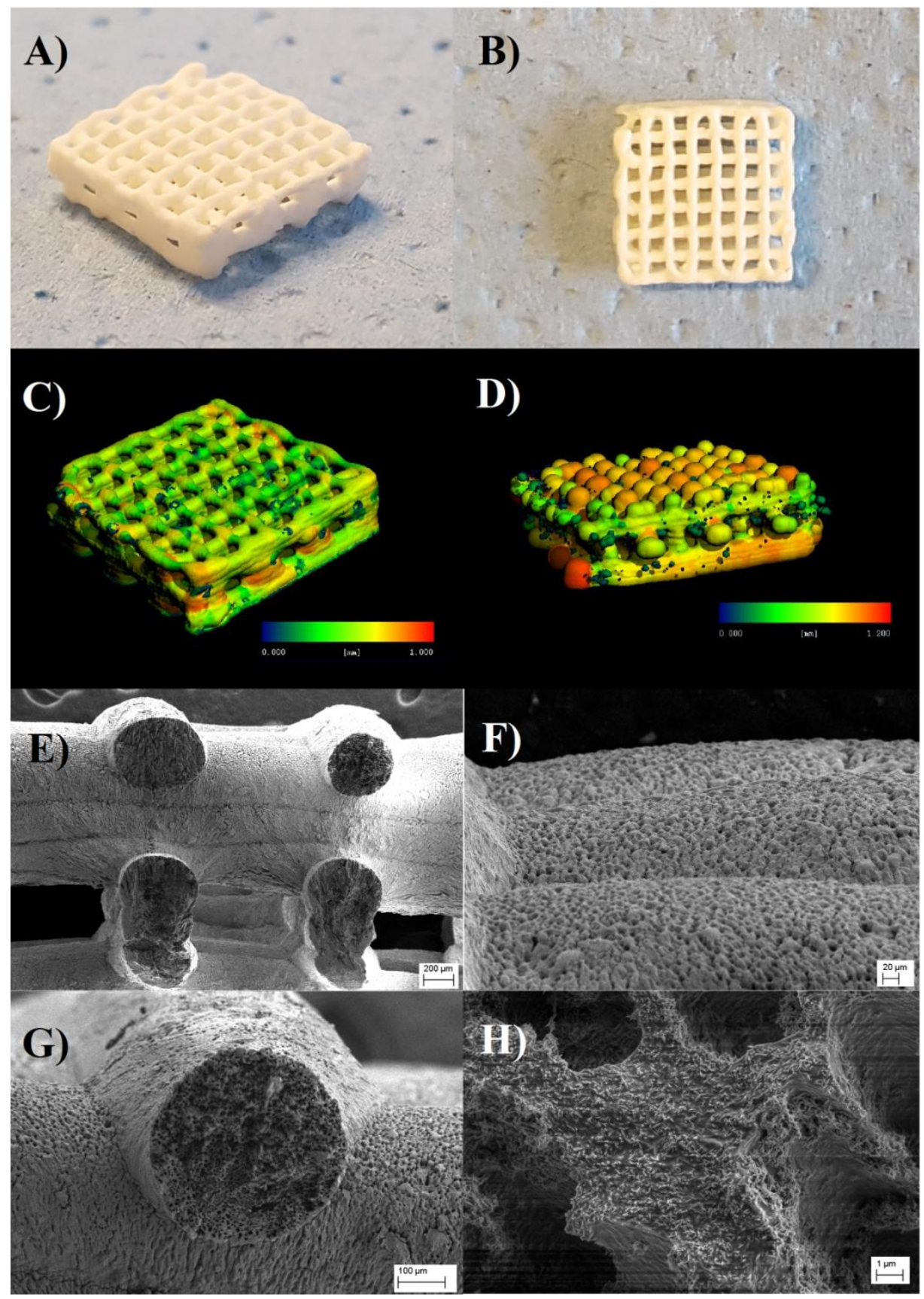

Figure 5. Images of PTMC-MA/nHA scaffolds (A, B), $\mu$ CT reconstructions of the trabecular $(C$, scale bar $=1$ $\mathrm{mm})$ and pore space $(D$, scale bar $=1.2 \mathrm{~mm})$ of the fabricated scaffolds and HR-SEM images of the cross-section of fabricated scaffolds $(E, G, H$, scale bars $=200,100$ and $1 \mu \mathrm{m}$ respectively) and of the surface $(F$, scale bar $=$ $20 \mu \mathrm{m})$. 


\section{Conclusions}

Using LTEAM inks we have prepared composite materials of photo-crosslinked PTMCMA with nHA, possessing a micro-porosity obtained through solvent crystallization and extraction. We have shown that the inks show favorable properties for extrusion-based additive manufacturing, such as shear thinning and a sharp crystallization response. We have furthermore shown that particulate solid drug compounds may be incorporated in the photo-crosslinked composite materials. Although affecting the mechanical properties, mechanically stable materials were formed. Additionally, drug release was sustained over at least four weeks. Using the PTMC-MA/nHA ink we have shown that it is possible to prepare designed structures by LTEAM with a micro-porosity in the struts and a homogeneous distribution of embedded nHA. These results show that inks based on PTMC-MA, nHA and EC are promising for the formation of micro-porous structures by LTEAM and that drugs may be incorporated and released from these structures. Currently we are working on optimization of the LTEAM process in order to fabricate structures for the evaluation of their biological performance.

\section{Acknowledgements}

The authors acknowledge the funding by the EU FP7 RAPIDOS project (Grant No. NMP3SL-2013-604517) and the financial support of the Stiftung für Innovation, Entwicklung und Forschung Graubünden for the purchase of the 3D Discovery system from RegenHU Ltd.. Furthermore the authors express their gratitude to Kuros BioSciences BV for their kind contribution of nano-hydroxyapatite.

\section{References}

1. Sachlos, E. and J.T. Czernuszka, Making tissue engineering scaffolds work. Review: the application of solid freeform fabrication technology to the production of tissue engineering scaffolds. European cells \& materials, 2003. 5: p. 29-39; discussion 39-40.

2. Loh, Q.L. and C. Choong, Three-dimensional scaffolds for tissue engineering applications: role of porosity and pore size. Tissue engineering Part B - Reviews, 2013. 19(6): p. 485-502.

3. Wüst, S., R. Muller, and S. Hofmann, Controlled Positioning of Cells in Biomaterials-Approaches Towards 3D Tissue Printing. Journal of functional biomaterials, 2011. 2(3): p. 119-154.

4. Do, A.V., B. Khorsand, S.M. Geary, and A.K. Salem, 3D Printing of Scaffolds for Tissue Regeneration Applications. Advanced healthcare materials, 2015. 4(12): p. 1742-1762.

5. Lantada, A.D. and P.L. Morgado, Rapid prototyping for biomedical engineering: current capabilities and challenges. Annual review of biomedical engineering, 2012. 14: p. 73-96. 
6. Chapter 4 of this thesis; Geven, M.A., V. Varjas, L. Kamer, X.J. Wang, J. Peng, D. Eglin, and D.W. Grijpma, Fabrication of patient specific composite orbital floor implants by stereolithography. Polymers for Advanced Technologies, 2015. 26(12): p. 1433-1438.

7. Betz, M.W., J.F. Caccamese, D.P. Coletti, J.J. Sauk, and J.P. Fisher, Challenges associated with regeneration of orbital floor bone. Tissue Engineering Part B: Reviews, 2010. 16(5): p. 541-550.

8. Jaquiery, C., C. Aeppli, P. Cornelius, A. Palmowsky, C. Kunz, and B. Hammer, Reconstruction of orbital wall defects: critical review of 72 patients. International Journal of Oral \& Maxillofacial Surgery, 2007. 36(3): p. 193-199.

9. Baino, F., Biomaterials and implants for orbital floor repair. Acta Biomaterialia, 2011. 7(9): p. 3248-3266.

10. Shim, J.H., J.Y. Kim, M. Park, J. Park, and D.W. Cho, Development of a hybrid scaffold with synthetic biomaterials and hydrogel using solid freeform fabrication technology. Biofabrication, 2011. 3(3): p. 034102.

11. Reichert, J.C., A. Heymer, A. Berner, J. Eulert, and U. Noth, Fabrication of polycaprolactone collagen hydrogel constructs seeded with mesenchymal stem cells for bone regeneration. Biomedical materials, 2009. 4(6): p. 065001.

12. Shor, L., S. Güçeri, R. Chang, J. Gordon, Q. Kang, L. Hartsock, Y. An, and W. Sun, Precision extruding deposition (PED) fabrication of polycaprolactone (PCL) scaffolds for bone tissue engineering. Biofabrication, 2009. 1(1): p. 015003.

13. Roh, H.S., S.W. Myung, S.-C. Jung, and B.-H. Kim, Fabrication of 3D Scaffolds with Nano-Hydroxyapatite for Improving the Preosteoblast Cell-Biological Performance. Journal of Nanoscience and Nanotechnology, 2015. 15(8): p. 55855588.

14. Vandrovcova, M., T.E.L. Douglas, W. Mroz, O. Musial, D. Schaubroeck, B. Budner, R. Syroka, P. Dubruel, and L. Bacakova, Pulsed laser deposition of magnesium-doped calcium phosphate coatings on porous polycaprolactone scaffolds produced by rapid prototyping. Materials Letters, 2015. 148: p. 178-183.

15. Sa, M.W., S.E. Kim, Y.P. Yun, H.R. Song, and J.Y. Kim, Fabrication of Hybrid Scaffolds by Polymer Deposition System and Its In-vivo Evaluation with a Rat Tibial Defect Model. Tissue Engineering and Regenerative Medicine, 2014. 11(6): p. 439-445.

16. Yeo, M., H. Lee, and G. Kim, Three-dimensional hierarchical composite scaffolds consisting of polycaprolactone, beta-tricalcium phosphate, and collagen nanofibers: fabrication, physical properties, and in vitro cell activity for bone tissue regeneration. Biomacromolecules, 2011. 12(2): p. 502-510.

17. Hsu, S.H., H.J. Yen, C.S. Tseng, C.S. Cheng, and C.L. Tsai, Evaluation of the growth of chondrocytes and osteoblasts seeded into precision scaffolds fabricated by fused deposition manufacturing. Journal of Biomedical Materials Research Part B - Applied Biomaterials, 2007. 80(2): p. 519-527. 
18. $\mathrm{Wu}, \mathrm{L}$. and J. Ding, In vitro degradation of three-dimensional porous poly $(D, L$ lactide-co-glycolide) scaffolds for tissue engineering. Biomaterials, 2004. 25(27): p. 5821-5830.

19. Lam, C.X., M.M. Savalani, S.H. Teoh, and D.W. Hutmacher, Dynamics of in vitro polymer degradation of polycaprolactone-based scaffolds: accelerated versus simulated physiological conditions. Biomedical materials, 2008. 3(3): p. 034108.

20. Zhang, Z., R. Kuijer, S.K. Bulstra, D.W. Grijpma, and J. Feijen, The in vivo and in vitro degradation behavior of poly(trimethylene carbonate). Biomaterials, 2006. 27(9): p. 1741-1748.

21. Zhu, K.J., R.W. Hendren, K. Jensen, and C.G. Pitt, Synthesis, Properties, and Biodegradation of Poly(1,3-Trimethylene Carbonate). Macromolecules, 1991. 24(8): p. 1736-1740.

22. Schuller-Ravoo, S., J. Feijen, and D.W. Grijpma, Flexible, elastic and tearresistant networks prepared by photo-crosslinking poly(trimethylene carbonate) macromers. Acta Biomaterialia, 2012. 8(10): p. 3576-3585.

23. Karageorgiou, V. and D. Kaplan, Porosity of $3 D$ biomaterial scaffolds and osteogenesis. Biomaterials, 2005. 26(27): p. 5474-5491.

24. Moroni, L., R. Licht, J. de Boer, J.R. de Wijn, and C.A. van Blitterswijk, Fiber diameter and texture of electrospun PEOT/PBT scaffolds influence human mesenchymal stem cell proliferation and morphology, and the release of incorporated compounds. Biomaterials, 2006. 27(28): p. 4911-4922.

25. Wang, A.J., T. Paterson, R. Owen, C. Sherborne, J. Dugan, J.M. Li, and F. Claeyssens, Photocurable high internal phase emulsions (HIPEs) containing hydroxyapatite for additive manufacture of tissue engineering scaffolds with multiscale porosity. Materials science \& engineering Part C - Materials for biological applications, 2016. 67: p. 51-58.

26. Alkoclar, E.C. and M. Yesil, Composition for treating an viral infection. 2013. p. 11.

27. Wei, S. and H. Wang, Composition for treating hyperplasia. 2015.

28. Chapter 3 of this thesis; Geven, M.A., D. Barbieri, H. Yuan, J.D. de Bruijn, and D.W. Grijpma, Preparation and mechanical properties of photo-crosslinked poly(trimethylene carbonate) and nano-hydroxyapatite composites. Clinical hemorheology and microcirculation, 2015. 60(1): p. 3-11.

29. Hildebrand, T. and P. Ruegsegger, A new method for the model-independent assessment of thickness in three-dimensional images. Journal of Microscopy, 1997. 185: p. 67-75.

30. Cordewener, F.W., F.R. Rozema, C.A.P. Joziasse, R.R.M. Bos, G. Boering, and A.J. Pennings, Poly(96l/4d-Lactide) Implants for Repair of Orbital Floor Defects an in-Vitro Study of the Material Properties in a Simulation of the Human Orbit. Journal of Materials Science-Materials in Medicine, 1995. 6(10): p. 561-568. 


\title{
Chapter 9 - Osteogenic differentiation of hBMSCs on porous photo-crosslinked poly(trimethylene carbonate) and nano- hydroxyapatite composites
}

Mike A. Geven ${ }^{\mathrm{a}}$, Anna Lapomarda ${ }^{\mathrm{b}}$, Olivier Guillaume ${ }^{\mathrm{c}}$, Christoph M. Sprecher ${ }^{\mathrm{c}}$, David Eglin $^{\mathrm{c}}$, Giovanni Vozzi ${ }^{\mathrm{b}}$ and Dirk W. Grijpma ${ }^{\mathrm{a}}$

${ }^{a}$ Department of Biomaterials Science and Technology, University of Twente, Enschede, The Netherlands

${ }^{\mathrm{b}}$ Research Center “E. Piaggio" and Dipartimento di Ingegneria dell'Informazione, University of Pisa, Pisa, Italy

${ }^{c}$ AO Research Institute, Davos, Switzerland

\begin{abstract}
.
Large bone defects are challenging to repair and novel implantable materials are needed to aid in their reconstruction. Research in the past years has proven the beneficial effect of porosity in an implant on osteogenesis in vivo. Building on this research we report here on porous composites based on photo-crosslinked poly(trimethylene carbonate) and nanohydroxyapatite. These composites were prepared by a temperature induced phase separation of poly(trimethylene carbonate) macromers from solution in ethylene carbonate. By controlling the ethylene carbonate content in viscous dispersions of nanohydroxyapatite in poly(trimethylene carbonate) macromer solutions, composites with 40wt.\% nano-hydroxyapatite and a varying porosity were prepared. The surface structure of these porous composites was affected by their porosity, the stiffness and toughness of the composites decreased with increasing porosity. In cell culture experiments, human bone marrow mesenchymal stem cells proliferated well on the composites irrespective of their porosity. There was an effect on the osteogenic differentiation of the cells (as indicated by changes in calcium production), however. This may be caused by the changes in the mechanical properties of the porous composites and a reduced ability of the cells to aggregate and to elongate into morphologies favourable for osteogenic differentiation on highly porous materials.
\end{abstract}




\section{Introduction}

In recent years in vitro and in vivo research in bone tissue engineering has increased the understanding of factors controlling the clinical outcome of reconstruction. It is known which size features are desired for the porosity of an implant[1-6], which surface microand nanostructure is efficacious[7-14], and how the mechanical properties [15-17] affect the osteogenicity and -integration of an implant. In practice several of these factors are interrelated and compromises are generally made, such as those between the mechanical properties and the porosity[18, 19].

The effect of porosity of implant materials on the clinical outcome of bone reconstruction has been researched extensively. The porosity of the implant material is essential for ingrowth of bone tissue and to allow for vascularization[18-22]. Porosity can additionally improve de novo bone ingrowth by enhancing the surface roughness of the implant, increasing the local protein concentration and by improving nutritional supply to the de novo bone that has grown into the implant[22-24]. The mechanical properties of the implant are of functional importance in bone restoration as adequate mechanical properties allow for the implant to act as a temporary support structure. Moreover, the osteogenic differentiation of osteoprogenitor cells is enhanced on materials with increasing stiffness[15, 16, 25]. Given the compromise between porosity and mechanical properties, these factors need to be balanced for effective restoration of a bony defect.

In this work we use a temperature induced phase separation method to prepare composites of photo-crosslinked PTMC and nHA with varying porosity. We used ethylene carbonate (EC, melting point $36{ }^{\circ} \mathrm{C}$ ) to dissolve the polymer phase and disperse the nHA at elevated temperatures. At reduced temperatures, phase separation occurs between the solvent and polymer phase. The solvent phase forms crystals which can be extracted after photocrosslinking to generate porosity. By varying the concentration of EC in resins of photocrosslinkable PTMC and nHA, we are able to adjust porosity, pore size and mechanical properties of composites of photo-crosslinked PTMC containing $40 \mathrm{wt} \% \mathrm{nHA}$. In previous work we have shown that such composites are useful for bone tissue engineering. These materials allow for good cell proliferation and differentiation on their surfaces in vitro, and they enhance reconstruction of calvarial defects in vivo in rabbits[26, 27]. By keeping the nHA content of the porous composites in this work more or less constant while the porosity is varied, we show the effect of the porosity on mechanical properties of the composites and on calcium release and protein adhesion as these factors play a further important role in bone restoration[27-29]. We furthermore show the effect of the various porous composites on adhesion, proliferation and the osteogenic differentiation of human bone marrow mesenchymal stem cells (hBMSCs) in vitro.

The fabrication method described here can be used to optimize composite materials for bone tissue engineering as it allows for direct attuning of the porosity and mechanical properties by only controlling the solvent concentration and fabrication temperatures during composite preparation. 


\section{Materials \& Methods}

\subsection{Materials}

Hiuzhou ForYou Medical Devices Ltd. provided the trimethylene carbonate (TMC) used. Tin(II) 2-ethylhexanoate $\left(\mathrm{Sn}(\mathrm{Oct})_{2}\right), \quad$ 1,1,1-tris(hydroxymethyl)propane (TMP), hydroquinone (HQ), methacrylic anhydride, triethylamine, ethylene carbonate, formic acid ( $\geq 95.5 \%$ ), sodium hydrogen carbonate, ascorbic acid, glycerol-2-phosphate (disodium salt hydrate), dexamethasone, phosphatase substrate, alkaline buffer solution (1.5M, pH 10.3), Live/Dead® staining reagents, alizarin red staining (ARS, $40 \mathrm{mM}$ ) solution, bovine serum albumin and a calcium colorimetric assay kit (MAK022) were acquired from Sigma Aldrich. Omnirad TPO-L photo-initiator was obtained from IMG Resins BV. Kuros BioSciences BV provided $\mathrm{nHA}$ in the form of a powder with aggregates $(\varnothing=15 \mu \mathrm{m})$ of needle-like hydroxyapatite crystals of 200 to $400 \mathrm{~nm}$ and 20 to $50 \mathrm{~nm}$ wide.

Tris(hydroxymethyl) aminomethane hydrochloride salt (Tris-HCL, > 99\%), diethanolamine and hexamethyldisiloxane (HMDS) were obtained from Carl Roth GmbH. Dulbecco's modified eagle medium (DMEM, low glucose, pyruvate) powder, minimum essential medium $\alpha(\alpha-$ MEM) and penicilin streptomycin $(10,000 \mathrm{U} / \mathrm{ml})$ were from Gibco. SeraPlus serum was acquired from Milan Analytica AG. A Quick Start ${ }^{\text {TM }}$ Bradford Protein Assay was from Bio-Rad. A CellTiter-Blue ${ }^{\circledR}$ assay was acquired from Promega and a CyQuant ${ }^{\mathrm{TM}}$ cell proliferation assay kit was used from Invitrogen. Nunc ${ }^{\mathrm{TM}}$ Thermanox ${ }^{\mathrm{TM}}$ coverslips were obtained from Thermofisher.

Dichloromethane was from VWR International and was dried over calcium hydride and subsequently distilled under a dry $\mathrm{N}_{2}$ atmosphere. Other materials were used as received unless stated otherwise. Water used for this work was either deionized (DI) water or ultrapure (UP) water purified by a MilliQ ${ }^{\circledR}$ Reference system. Phosphate buffered saline (PBS) used in this work was a $0.01 \mathrm{M}$ phosphate buffer at $\mathrm{pH}$ 7.4.

\subsection{Synthesis of photo-crosslinkable PTMC}

Three-armed methacrylate end-group functionalized poly(trimethylene carbonate) (PTMCMA) was synthesized as previously described[30]. Briefly, a three-armed PTMC oligomer was prepared by ring-opening polymerization of TMC initiated by TMP using $\mathrm{Sn}(\mathrm{Oct})_{2}$ $(0.13$ wt.\%) as a catalyst (molar ratio TMC/TMP $=96.2)$. The polymerization reaction was conducted for three days at $130{ }^{\circ} \mathrm{C}$ under $\mathrm{N}_{2}$ atmosphere. Thereafter the oligomer was dissolved in dry dichloromethane under $\mathrm{N}_{2}$. To this solution HQ at $0.1 \mathrm{wt} . \%$ relative to PTMC oligomer, triethylamine (9 mol/mol PTMC oligomer) and methacrylic anhydride (9 $\mathrm{mol} / \mathrm{mol}$ PTMC oligomer) was subsequently added under stirring to functionalize the oligomer with methacrylate end-groups. The functionalization was left to proceed for 5 days at room temperature under $\mathrm{N}_{2}$ atmosphere. The resulting solution was precipitated in ice-cooled methanol and the precipitate was additionally extracted in ice-cooled methanol for 2 hours. It was thereafter dried in vacuo at room temperature until constant weight. The resulting macromer was analysed by ${ }^{1} \mathrm{H}-\mathrm{NMR}$ (Bruker Ascend 400/Avance III $400 \mathrm{MHz}$ 
NMR spectrometer) to determine the molar mass and degree of functionalization as was described previously[31]. Deuterated chloroform was used as a solvent for ${ }^{1} \mathrm{H}-\mathrm{NMR}$.

\subsection{Preparation of resins and composite films}

Resins to produce porous composite films were prepared by adding nHA and PTMC-MA into chloroform at a ratio of 0.67 (nHA/PTMC-MA). The concentration of PTMC-MA was $0.1 \mathrm{~g} / \mathrm{ml}$. This mixture was stirred overnight in the dark to dissolve the PTMC-MA. The resulting dispersion was co-precipitated in ice-cooled methanol and the collected precipitate was dried in vacuo until constant weight. After drying, the precipitate was mixed with a calculated amount of $\mathrm{EC}$ at $50{ }^{\circ} \mathrm{C}$ for two days in the dark. This mixture was sonicated at $50{ }^{\circ} \mathrm{C}$ for 30 minutes and HQ at $0.1 \mathrm{wt} . \%$ relative to PTMC-MA and Omnirad TPO-L at 5 wt.\% relative to PTMC-MA were mixed-in directly after.

Three different concentrations of EC were used in order to prepare resins for the production of films with 35, 60 and 85\% porosity (LowEC, MidEC and HighEC respectively). The final concentration of all components in these three resins is depicted in table 1.

Porous composite films were prepared by casting resins at a thickness of $0.5 \mathrm{~mm}$ on a heated glass plate. The glass plate was then cooled for 2 hours in a refrigerator or freezer to induce a phase separation. The casting and cooling temperatures applied are given in table 2. As the concentration of EC in the resins influenced the rate of EC crystallization and the size of EC crystals, these temperatures were adjusted to control the cooling rate. Films were then photo-crosslinked in a UV-crosslinking box at $365 \mathrm{~nm}$ and $11 \mathrm{~mW} / \mathrm{cm}^{2}$ under $\mathrm{N}_{2}$ atmosphere for 30 minutes at room temperature. The EC was thereafter extracted in deionized water for 24 hours and subsequently in a mixture of ethanol and propylene carbonate $(1 / 1 \mathrm{v} / \mathrm{v})$ which was exchanged to pure ethanol stepwise. The films were subsequently dried under ambient conditions for 3 days. The thickness of the dried films was between 0.30 and $0.45 \mathrm{~mm}$.

Table 1. Composition of resins used for this work.

\begin{tabular}{lccc}
\hline & \multicolumn{3}{c}{ Resins } \\
\cline { 2 - 4 } Component & LowEC & MidEC & HighEC \\
\hline PTMC-MA (wt.\%) & 41.5 & 28.1 & 11.1 \\
nHA (wt.\%) & 27.7 & 18.7 & 7.4 \\
EC (wt.\%) & 28.7 & 51.8 & 81.0 \\
Omnirad TPO-L (wt.\%) & 2.1 & 1.4 & 0.5 \\
HQ (wt.\%) & $4 \cdot 10^{-2}$ & $3 \cdot 10^{-2}$ & $1 \cdot 10^{-2}$ \\
\hline
\end{tabular}


Table 2. Temperatures applied for resin casting and for cooling

\begin{tabular}{lccc}
\hline & \multicolumn{3}{c}{ Resins } \\
\cline { 2 - 4 } Applied temperature & LowEC & MidEC & HighEC \\
\hline Casting $\left({ }^{\circ} \mathrm{C}\right)$ & 50 & 50 & 40 \\
Cooling $\left({ }^{\circ} \mathrm{C}\right)$ & -25 & 4 & 4 \\
\hline
\end{tabular}

\subsection{Characterization of resins and porous composite films}

Melting- and crystallization temperatures of the resins were determined by differential scanning calorimetry (DSC) using a Perkin Elmer Pyris 1 differential scanning calorimeter. Samples $(n=3)$ were heated from -45 to $75{ }^{\circ} \mathrm{C}$ under $\mathrm{N}_{2}$ atmosphere using a heating rate of $5{ }^{\circ} \mathrm{C} /$ minute. Subsequently the samples were kept at $75^{\circ} \mathrm{C}$ for 30 minutes after which they were cooled to $-45{ }^{\circ} \mathrm{C}$ at a rate of $5{ }^{\circ} \mathrm{C} /$ minute. The samples were kept at $-45{ }^{\circ} \mathrm{C}$ for 30 minutes after which a second, identical heating and cooling cycle was performed. The melting temperature of the resins was determined as the peak temperature of the first heating cycle, whereas the crystallization temperature was determined as the peak temperature in the second cooling cycle.

The viscosity of the resins was determined by rheology on an Anton Paar Physica MCR201 rheometer equipped with PP25 measurement probe with a $25 \mathrm{~mm}$ diameter. Measurements were performed at the casting temperatures of the resins and a measurement gap of $0.3 \mathrm{~mm}$. To equilibrate samples, a pre-shear of $10 \mathrm{~s}^{-1}$ for 5 minutes and $0.01 \mathrm{~s}^{-1}$ for 2 minutes was applied. The shear rate was thereafter increased from 0.01 to $100 \mathrm{~s}^{-1}$ at 30 points per decade and $6 \mathrm{~s}$ in-between measuring points.

The surface and cross-sections of freeze-fractured films were visualized using a Zeiss Merlin high resolution scanning electron microscope (HR-SEM) equipped with a high efficiency scanning electron detector at 1.4 to $5 \mathrm{kV}$. Pore width was determined from the SEM images by ImageJ. At least 300 randomly selected pores per film were analysed.

The surface of the films was further characterized by atomic force microscopy (AFM) using a Multi Mode atomic force microscope from Veeco. Images were acquired in tapping mode at $1.01 \mathrm{~Hz}$ and a scan angle of $90^{\circ}$. The mean roughness $\left(\mathrm{R}_{\mathrm{a}}\right)$ as well as root mean square roughness $\left(\mathrm{R}_{\mathrm{q}}\right)$ were calculated as defined in literature[32].

The nHA content of the composite films was determined by thermogravimetric analysis (TGA) using a Perkin Elmer TGA 7. Samples $(n=3)$ were heated under $\mathrm{N}_{2}$ flow from 50 to $600{ }^{\circ} \mathrm{C}$ at a heating rate of $20{ }^{\circ} \mathrm{C} /$ minute. The nHA content was determined from the residual mass of the sample at $600{ }^{\circ} \mathrm{C}$.

To calculate the porosity of the composite films, samples $(\mathrm{n}=3, \emptyset=6 \mathrm{~mm})$ were die-cut from the films and their weight $\left(\mathrm{m}_{\mathrm{s}}\right)$ and thickness was determined. The volumetric porosity (P) in \% was thereafter calculated using (1), where $\mathrm{f}_{\text {PTMC }}$ and $\mathrm{f}_{\mathrm{nHA}}$ are the weight fractions of PTMC and nHA respectively as was determined by TGA. The densities of PTMC-MA $\left(1.31 \mathrm{~g} / \mathrm{cm}^{3}\right)$ and nHA $\left(3.162 \mathrm{~g} / \mathrm{cm}^{3}\right)$ are $\rho_{\text {PTMC }}$ and $\rho_{\text {nHA }}$ respectively and $V_{\mathrm{s}}$ is the overall volume of the sample in $\mathrm{cm}^{3}$ as was determined from its diameter and thickness. 


$$
P=\left[1-\frac{m_{S}}{\left(f_{P T M C} * \rho_{P T M C}+f_{n H A} \rho_{n H A}\right) V_{S}}\right] * 100 \%
$$

The degree of swelling of the composite films in chloroform was determined by placing disk-shaped samples $(\mathrm{n}=3, \varnothing=6 \mathrm{~mm})$ in chloroform for 24 hours at room temperature. Samples were weighed before immersion $\left(\mathrm{m}_{\mathrm{i}}\right)$ and their thickness was determined. After immersion and blotting their surface, the wet weight of the samples was determined $\left(\mathrm{m}_{\mathrm{w}}\right)$ and the degree of swelling was calculated using (2). In (2), S is the degree of swelling in $\%$, $\rho_{\mathrm{c}}$ is the density of chloroform $\left(1.49 \mathrm{~g} / \mathrm{cm}^{3}\right)$ and $V_{\mathrm{s}}$ is the overall sample volume in $\mathrm{cm}^{3}$ before immersion as was determined from the sample diameter and thickness.

$$
S=\frac{m_{w}-m_{i}}{\rho_{c} V_{S}} * 100 \%
$$

The mechanical properties of the composite films were determined in tensile tests on a Zwick Z020 tensile tester equipped with a $500 \mathrm{~N}$ load cell. Film samples (at least $\mathrm{n}=5$ ) were die-cut into $100 \times 50 \mathrm{~mm}^{2}$ strips. An initial grip-to-grip separation of $50 \mathrm{~mm}$ and a crosshead speed of $50 \mathrm{~mm} /$ minute were applied during sample elongation at ambient conditions. Engineering stress-strain curves were obtained and the E-modulus was determined between 0 and $2 \%$ strain. Furthermore, the yield point, ultimate strength and ultimate strain were determined. Additionally, the toughness was determined from the area under the stress-strain curves.

Calcium release from the porous composite films was determined using square samples (n $=3$ ) of $1 \times 1 \mathrm{~cm}^{2}$. These were incubated in a formic acid buffer containing $133 \mathrm{mM} \mathrm{NaCl}$ $(\mathrm{pH}=4.5 \pm 0.05)$ at $37^{\circ} \mathrm{C}$. At selected time points the release medium was completely replaced by fresh medium. To determine the calcium content in the removed medium, a calcium colorimetric assay kit was used according to the supplier's protocol. After treatment by the assay kit, calcium ion concentration was determined by absorbance at 560 nm.

Protein adsorption on the porous composite films was investigated by incubation of film samples $(\mathrm{n}=4, \varnothing=1.1 \mathrm{~cm})$ in PBS containing $10 \%(\mathrm{v} / \mathrm{v})$ SeraPlus and $0.02 \%(\mathrm{w} / \mathrm{v})$ sodium azide at $37{ }^{\circ} \mathrm{C}$ for 5 days. Samples were thereafter washed with DI water and mixed with $0.05 \%(\mathrm{v} / \mathrm{v})$ Triton ${ }^{\mathrm{TM}} \mathrm{X}-100$ in UP water for 30 minutes at room temperature. The resulting supernatant was diluted $5 \mathrm{x}$ with DI water to reduce the Triton ${ }^{\mathrm{TM}} \mathrm{X}-100$ concentration to $0.01 \%$ (v/v). Subsequent analysis was performed using a Quick Start ${ }^{\mathrm{TM}}$ Bradford Protein Assay according to the supplier's protocol. Bovine serum albumin was used as reference. Protein concentration was quantified by absorbance at $595 \mathrm{~nm}$ after treatment by the assay kit.

Absorbance was recorded on a Viktor ${ }^{3}$ microplate reader of Perkin-Elmer for calcium and protein assays. 


\subsection{In vitro culture and differentiation of hBMSCs}

The hBMSCs used for this work were obtained from vertebral body bone aspirates of two separate donors (undergoing spinal fusion; obtained with informed consent and full ethical approval [KEK Bern 126/03]) as described previously[27, 33]. Before seeding, hBMSCs of both donors were expanded separately in $\alpha$-MEM medium containing $10 \%(\mathrm{v} / \mathrm{v})$ of SeraPlus.

Disk-shaped composite film samples covering the bottom of wells-plates were used in cell culture experiments. The film samples were sterilized using a cold ethylene oxide gas treatment and were thereafter kept under vacuum for 5 days until use. Tissue culture poly(styrene) (TCPS) and Thermanox ${ }^{\mathrm{TM}}$ coverslips were used as positive controls in some experiments. Before seeding the hBMSCs, a pre-incubation of the samples and controls was performed overnight in full osteogenic medium (OM) consisting of a solution of DMEM powder $(1 \mathrm{~g} / \mathrm{ml})$, sodium hydrogen carbonate $(4 \mathrm{mg} / \mathrm{ml})$, penicilin streptomycin $(1 \%[\mathrm{v} / \mathrm{v}])$, SeraPlus $(10 \%[\mathrm{v} / \mathrm{v}])$, ascorbic acid $(50 \mu \mathrm{g} / \mathrm{ml})$, dexamethasone $(10 \mathrm{nM})$ and glycerol-2-phosphate $(5 \mathrm{mM})$ in UP water.

Pre-incubation and the culture of cells of both donors in $\mathrm{OM}$ were performed at $37{ }^{\circ} \mathrm{C}, 5 \%$ $\mathrm{CO}_{2}$ and $90 \%$ relative humidity. The cells of both donors were seeded separately at a density of $21 \cdot 10^{3}$ cells $/ \mathrm{cm}^{2}$ unless stated otherwise. Medium was exchanged twice per week.

An adhesion assay of hBMSCs on film samples $(n=3)$ was performed by seeding hBMSCs at a density of $135 \cdot 10^{3}$ cells $/ \mathrm{cm}^{2}$ in $30 \mu \mathrm{l}$ of OM. Samples were then incubated for 15,30 and 60 minutes under cell culture conditions without further addition of medium. The cells were thereafter lysed by incubation at $4{ }^{\circ} \mathrm{C}$ for 2.5 hours in cell lysis buffer consisting of Triton $^{\mathrm{TM}} \mathrm{X}-100$ at $0.1 \%(\mathrm{v} / \mathrm{v})$ and Tris- $\mathrm{HCl}$ at $10 \mathrm{mM}(\mathrm{pH} 7.4)$ in DI water. Cell lysates were stored at $-80{ }^{\circ} \mathrm{C}$ and analysed after thawing, using a CyQuant ${ }^{\mathrm{TM}}$ cell proliferation kit according to the supplier's protocol.

The metabolic activity of the hBMSCs was monitored using a CellTiter-Blue ${ }^{\circledR}$ assay. At designated time points, seeded samples were washed and incubated in a CellTiter-Blue ${ }^{\circledR}$ solution in $\mathrm{OM}$ at $20 \%(\mathrm{v} / \mathrm{v})$ for 3 hours under cell culture conditions. The incubated CellTiter-Blue ${ }^{\circledR}$ solution was thereafter analysed according to the supplier's recommendations.

A Live/Dead® staining was performed after 1 and 28 days of culture. Seeded film samples $(n=2)$ were incubated for 1 hour under cell culture conditions in OM containing Live/Dead ${ }^{\circledR}$ staining reagents (calcein acetoxymethyl ester and ethidium homodimer at 10 and $1 \mu \mathrm{g} / \mathrm{ml}$ respectively). Stained cells were thereafter imaged using a Zeiss Axiovert 200 $m$ fluorescent confocal microscope equipped with an Axiocam HRc.

After 28 days of culture, cells on samples $(n=2)$ were visualized by SEM. Cells were fixated overnight using a $4 \%(\mathrm{w} / \mathrm{v})$ buffered paraformaldehyde solution at $4{ }^{\circ} \mathrm{C}$. The samples were subsequently dehydrated by ethanol/DI water mixtures which were slowly exchanged to $100 \%$ ethanol. Thereafter samples were treated by mixtures of ethanol/HDMS which were exchanged to $100 \%$ HDMS. Following the HDMS treatment 
the samples were dried under ambient conditions for 3 days, sputter-coated with a goldpalladium layer and imaged using a Hitachi S4700 FESEM at $3 \mathrm{kV}$.

The ALP activity of hBMSCs was determined after incubation of seeded samples $(n=3)$ in cell lysis buffer (v.s.). Cell lysates were stored at $-80{ }^{\circ} \mathrm{C}$ and after thawing the lysates were mixed with DI water, alkaline buffer solution and a phosphatase substrate buffer $(25 \mathrm{mg} / \mathrm{ml}$ in $1 \mathrm{M}$ diethanolamine buffer in $0.5 \mathrm{mM}$ magnesium chloride in DI water, $\mathrm{pH} 9.8$ ). The resulting mixtures were incubated at $37{ }^{\circ} \mathrm{C}$ for exactly 15 minutes and thereafter diluted two times with a $0.1 \mathrm{M} \mathrm{NaOH}$ solution. Reference solutions of 0 to $700 \mu \mathrm{M}$ p-nitrophenol in cell lysis buffer were identically treated as reference. The resulting solutions were analyzed by absorbance at $405 \mathrm{~nm}$. Another part of the cell lysates was used for DNA quantification as described (v.s.). ALP activity was expressed as enzyme activity in mol per minute of incubation time per $\mathrm{g}$ of DNA content.

Calcium production by the cells was quantified by ARS. Cells on samples $(n=3)$ were fixated with a $4 \%(\mathrm{w} / \mathrm{v})$ formaldehyde solution at room temperature for 15 minutes. The samples were thereafter incubated with ARS solution at $\mathrm{pH} 4.2$ for 1 hour at room temperature. Samples were subsequently washed with DI water for 5 days to remove unbound ARS. The remaining staining was extracted using a $10 \%(\mathrm{v} / \mathrm{v})$ solution of acetic acid for 30 minutes at room temperature. The supernatant was heated to $85{ }^{\circ} \mathrm{C}$ for 10 minutes and subsequently cooled on ice. After centrifugation the supernatant was treated by ammonium hydroxide to a $\mathrm{pH}$ of 4.1-4.5. Absorbance of the resulting solutions was recorded at $405 \mathrm{~nm}$. ARS concentrations were determined using reference solutions of 0 $2000 \mathrm{mM}$ ARS in an acetic acid and ammonium hydroxide buffer at $\mathrm{pH}$ 4.3. Negative control samples without cells were treated identically and their ARS content was subtracted from the ARS content of seeded samples.

For all assays the absorbance or fluorescence was measured on a Viktor ${ }^{3}$ microplate reader of Perkin-Elmer. All results of cell culture and differentiation experiments are expressed as a pooled average \pm standard deviation of both donors.

\section{Results \& discussion}

\subsection{Resins and porous composites}

Composite resins were prepared from mixtures of PTMC-MA, nHA and EC. Using ${ }^{1} \mathrm{H}-$ NMR it was determined that the PTMC-MA macromer had a molecular weight of 10.6 $\mathrm{kg} / \mathrm{mol}$ and a degree of functionalization of $>99 \%$.

The viscosity of the resins was determined in rheology experiments. Between a shear rate of 0.01 and $100 \mathrm{~s}^{-1}$ the viscosity of the LowEC, MidEC and HighEC resins was between $42 \cdot 10^{3}$ and $60 \mathrm{~Pa} \cdot \mathrm{s}, 7 \cdot 10^{3}$ and $4.5 \mathrm{~Pa} \cdot \mathrm{s}$ and 3.3 and $0.05 \mathrm{~Pa} \cdot \mathrm{s}$ respectively. Although the increasing EC content decreased the viscosity of the resin, all resins were shear thinning. The melting and crystallization temperatures of the resins were determined in DSC experiments. The melting temperature of the LowEC, MidEC and HighEC resins was $23.7 \pm 0.3{ }^{\circ} \mathrm{C}, 34.8 \pm 1.0{ }^{\circ} \mathrm{C}$ and $41.7 \pm 0.3{ }^{\circ} \mathrm{C}$, respectively. The crystallization temperature of the MidEC and HighEC resins was $12.9 \pm 7.5^{\circ} \mathrm{C}$ and $26.7 \pm 2.6^{\circ} \mathrm{C}$. No crystallization peak 
was found for the LowEC resin in DSC experiments. To ensure that the resins could be readily cast, temperatures were applied at or well above their melting temperatures depending on their viscosity. The resins were thereafter cooled to temperatures below their crystallization temperature to ensure the formation of EC crystals. In case of the LowEC resin a cooling temperature of $-25{ }^{\circ} \mathrm{C}$ was applied for 2 hours. Crystallization of EC was thereafter confirmed by a transition in appearance of the resin from glistening to matte.

The effective crystallization of EC in all resins after cooling was further confirmed by the porosity of the resulting photo-crosslinked composite films after EC extraction. The porosity was determined gravimetrically and was $27 \pm 3 \%, 52 \pm 4 \%$ and $71 \pm 5 \%$ for films prepared from LowEC, MidEC and HighEC resins respectively. Clearly, the porosity of the composites increased with increasing EC concentration in the resins, although porosities were lower than intended $(35 \%, 60 \%$ and $85 \%$ for the LowEC, MidEC and HighEC resin, respectively). This may be caused by partial collapse of the pores after EC extraction.

Figure 1 depicts SEM and AFM images of the surface of the composites as well as SEM images of the cross-section, depicting the presence of pores in the composites.

In figure $1 \mathrm{a}$ and $\mathrm{d}$ it is apparent that the composites with $27 \%$ porosity possessed a relatively low amount of pores. In the cross-section only very small, isolated pores with a width of less than $0.5 \mu \mathrm{m}$ could be found in high magnification images (insert figure 1d). Composites with $52 \%$ and $71 \%$ porosity possessed a rougher surface morphology due to the larger amount of pores on the surface, as can be seen in figure $1 \mathrm{~b}$ and c. Furthermore, the cross-sections of these composites contained pores throughout as is shown in figure 1e and $\mathrm{f}$. The orientation of the pores in these composites was heterogeneous through the cross-section as well as on the surface.

From SEM images, the pore width of pores on the composite film surfaces and in the crosssections was determined. Table 3 gives an overview of the pore width distribution of the composite films.

From the pore width distributions given in table 3 it can be seen that the average pore widths on the surface of the films are close although the distribution is different. Composites with $52 \%$ porosity possess the most confined distribution between 0.3 and 24.5 $\mu \mathrm{m}$, whereas composites with $71 \%$ porosity possess the widest distribution with some pores exceeding $100 \mu \mathrm{m}$ in width. In the cross-section of the composite films, the pore width increases and the size distribution increases with increasing porosity (and thus with the amount of EC used during preparation). The content of EC, along with the applied cooling rate, influences the nucleation and crystal growth rate of EC crystals upon cooling of the composite resins. This directly affects the size of the resulting EC crystals[34]. The differences in pore width distribution in the resulting photo-crosslinked composites may therefore be minimized by controlling the cooling rate of the resins carefully. 

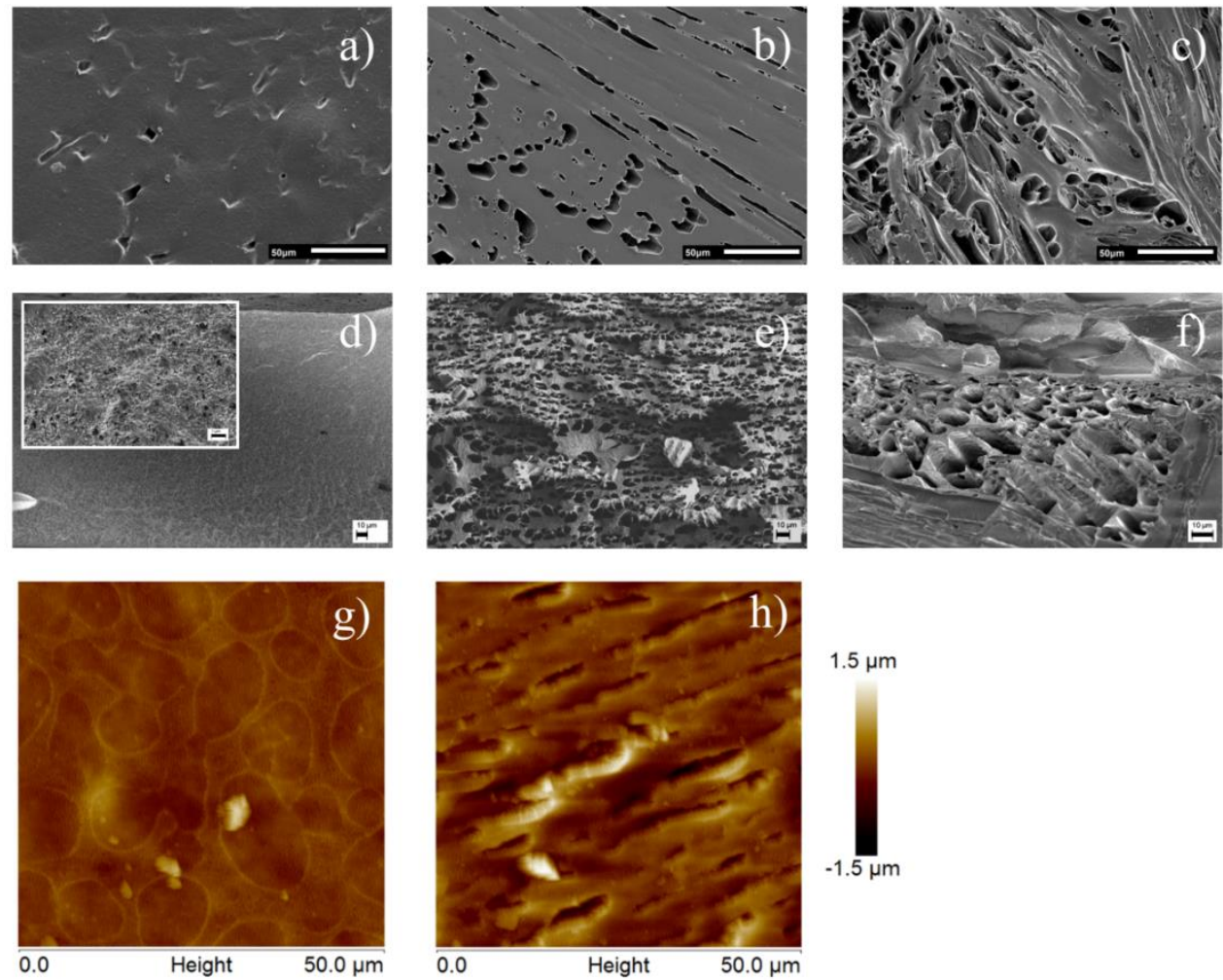

Figure 1. SEM images of the surface $(a, b, c)$ and cross-section $(d, e, f)$ of composite films with $27 \%(a, d), 52 \%$ $(b, e)$ and $71 \%(c, f)$ porosity and AFM images of composite films with $27 \%(g)$ and $52 \%(h)$ porosity. The surface of composites with $71 \%$ porosity could not be analysed by AFM due to large height differences. Composites with $27 \%, 52 \%$ and $71 \%$ porosity were prepared from LowEC, MidEC and HighEC resin respectively. Scale bars $a, b$, $c=50 \mu \mathrm{m}$; scale bars $d, e, f=10 \mu \mathrm{m} ;$ scale bar insert $d=1 \mu \mathrm{m}$.

Table 3. Pore width distribution on the surface and in the cross-section of porous composite films.

\begin{tabular}{llccc}
\hline & & \multicolumn{3}{c}{ Porosity } \\
\cline { 3 - 5 } Location & Pore width & $27 \%$ & $52 \%$ & $71 \%$ \\
\hline \multirow{3}{*}{ Surface } & Average $(\mu \mathrm{m})$ & 8.6 & 4.7 & 8.5 \\
& Minimum $(\mu \mathrm{m})$ & 2.4 & 0.3 & 0.9 \\
& Maximum $(\mu \mathrm{m})$ & 44.8 & 24.5 & 105 \\
\hline \multirow{3}{*}{ Cross-section } & Average $(\mu \mathrm{m})$ & 0.11 & 12.4 & 19.7 \\
& Minimum $(\mu \mathrm{m})$ & 0.02 & 1.8 & 0.3 \\
& Maximum $(\mu \mathrm{m})$ & 0.47 & 54.9 & 120 \\
\hline
\end{tabular}

In figure $1 \mathrm{~g}$ and $1 \mathrm{~h}$ AFM images of composites with $27 \%$ and $52 \%$ porosity are shown and it was observed that roughness increased with increasing porosity. From the AFM images an $R_{a}$ of $82 \pm 11 \mathrm{~nm}$ and $R_{q}$ of $107 \pm 21 \mathrm{~nm}$ were determined for composites with $27 \%$ 
porosity. The $\mathrm{R}_{\mathrm{a}}$ of composites with $52 \%$ porosity was $213 \pm 49 \mathrm{~nm}$ and the $\mathrm{R}_{\mathrm{q}}$ value was $279 \pm 60 \mathrm{~nm}$. In literature it has been shown that cells tend to respond strongly to roughness on the submicron and nanometer scale[8-10]. In our case, both micron scale and nano-scale features are present on the surface of our composites. Since the roughness remains in the range of hundreds of nanometres, however, the effect on the osteogenic differentiation of hBMSCs may be limited.

The porous composite films were further characterized in terms of their nHA content, degree of swelling in chloroform and their mechanical properties. An overview of these characteristics is given in table 4.

Table 4. Characteristics of the prepared porous composite films.

\begin{tabular}{lccc}
\hline & \multicolumn{3}{c}{ Porosity } \\
\cline { 2 - 4 } Property & $27 \%$ & $52 \%$ & $71 \%$ \\
\hline nHA content $(w t . \%)$ & $41.2 \pm 0.2$ & $48.8 \pm 2.2$ & $40.3 \pm 1.2$ \\
Degree of swelling (\%) & $202 \pm 20$ & $346 \pm 28$ & $663 \pm 102$ \\
E modulus (MPa) & $67.4 \pm 3.5$ & $28.0 \pm 4.3$ & $3.53 \pm 1.37$ \\
Yield strength $\left(\mathrm{N} / \mathrm{mm}^{2}\right)$ & $1.8 \pm 0.1$ & $0.8 \pm 0.1$ & $0.12 \pm 0.04$ \\
Yield strain $(\%)$ & $2.4 \pm 0.03$ & $2.7 \pm 0.07$ & $3.2 \pm 0.4$ \\
Ultimate tensile strength $\left(\mathrm{N} / \mathrm{mm}^{2}\right)$ & $5.3 \pm 1.2$ & $1.9 \pm 0.2$ & $0.26 \pm 0.05$ \\
Strain at break $(\%)$ & $66.8 \pm 23.8$ & $24.1 \pm 2.3$ & $13.4 \pm 4.2$ \\
Toughness $\left(\mathrm{N} / \mathrm{mm}^{2}\right)$ & $263 \pm 134$ & $31.2 \pm 5.2$ & $2.18 \pm 0.74$ \\
\hline
\end{tabular}

The nHA content in the composites was between 40 and 50 wt.\%. Differences observed in the nHA content did not correlate to the EC contents in the resins, indicating that it did not significantly affect nHA incorporation or distribution. With the increasing porosity of the composites the degree of swelling in chloroform was increased due to the enhanced pore volume.

From tensile mechanical properties in table 4 it is clear that the composites become less stiff as their porosity is increased. Composites with $27 \%$ porosity possess a relatively high stiffness, ultimate tensile strength and toughness. Composites with $71 \%$ porosity possess an almost twenty-fold lower stiffness and ultimate tensile strength and hundred-fold lower toughness.

A calcium release experiment was performed in a formic acid buffer $(\mathrm{pH}=4.5)$ to obtain an indication of the effect of the different porosities on the calcium release. Figure 2 shows the determined calcium release profile. 

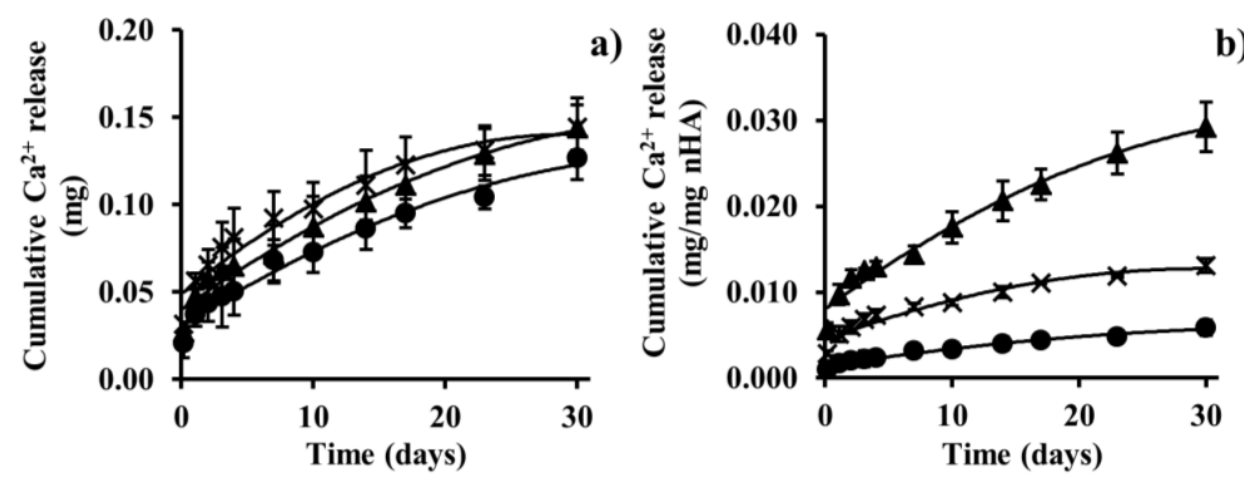

Figure 2. Cumulative calcium release from porous composite films (a) and cumulative release relative to the total $n H A$ content in the films (b) with $27 \%$ (dots), $52 \%$ (crosses) and $71 \%$ (triangles) porosity. Lines in graphs are shown to guide the eye.

In figure $2 \mathrm{a}$ it can be seen that calcium release from all porous composites is similar. Over 30 days there was no significant difference in the amount of calcium released. The amount of composite per unit volume in samples with high porosity is much lower than in those with low porosity however. Thus, when comparing the amount of calcium ions released to the total initial nHA content in each composite film, it is clear that the composite with $71 \%$ porosity more rapidly releases its calcium content compared to the other composites. This is illustrated in figure $2 \mathrm{~b}$. This may explain the similar overall release rates observed in figure 2a. Given this similarity, it is unlikely that it will affect osteogenic differentiation of hBMSCs.

The protein adsorption on the porous composites films was determined as well, and it was found that the porous composites with $27 \%, 52 \%$ and $71 \%$ porosity had a protein adhesion of $1.3 \pm 0.3,8.6 \pm 1.4$ and $15.6 \pm 2.2 \mu \mathrm{g} / \mathrm{sample}$ respectively. This can be expected as the surface area of the composites increases with increasing porosity and it may be beneficial for cell adherence and differentiation[27, 28].

\subsection{Proliferation and osteogenic differentiation of hBMSCs on porous composite films}

An adhesion study of hBMSCs on the composite films was performed to investigate the effect of the varying porosity and surface morphology on the initial binding of cells on the composites. In figure $3 \mathrm{a}$ the amount of DNA of adhering cells per composite film sample is shown. As a positive control TCPS is shown as well. On all films cells were adhering well. Although differences in adherence on the composites with varying porosity were observed, these became smaller over prolonged adhesion times. After 60 minutes of incubation, cells on all films showed similar adherence as on the TCPS control. Only on composites with $27 \%$ porosity the adhesion is slightly lower than on the other composites.

Continued cell proliferation was monitored by their metabolic activity. It is shown in figure $3 \mathrm{~b}$, that the hBMSCs grow well as their metabolic activity increases over time up to 13 
days of culture. Thereafter a decrease of the metabolic activity was observed on all samples including the TCPS control. This may be related to the confluence as well as the differentiation of the cells[35]. The metabolic activity was initially highest on the composite with $27 \%$ porosity and was comparable to that of the TCPS control. After 28 days of culture the lowest metabolic activity was observed on this composite.
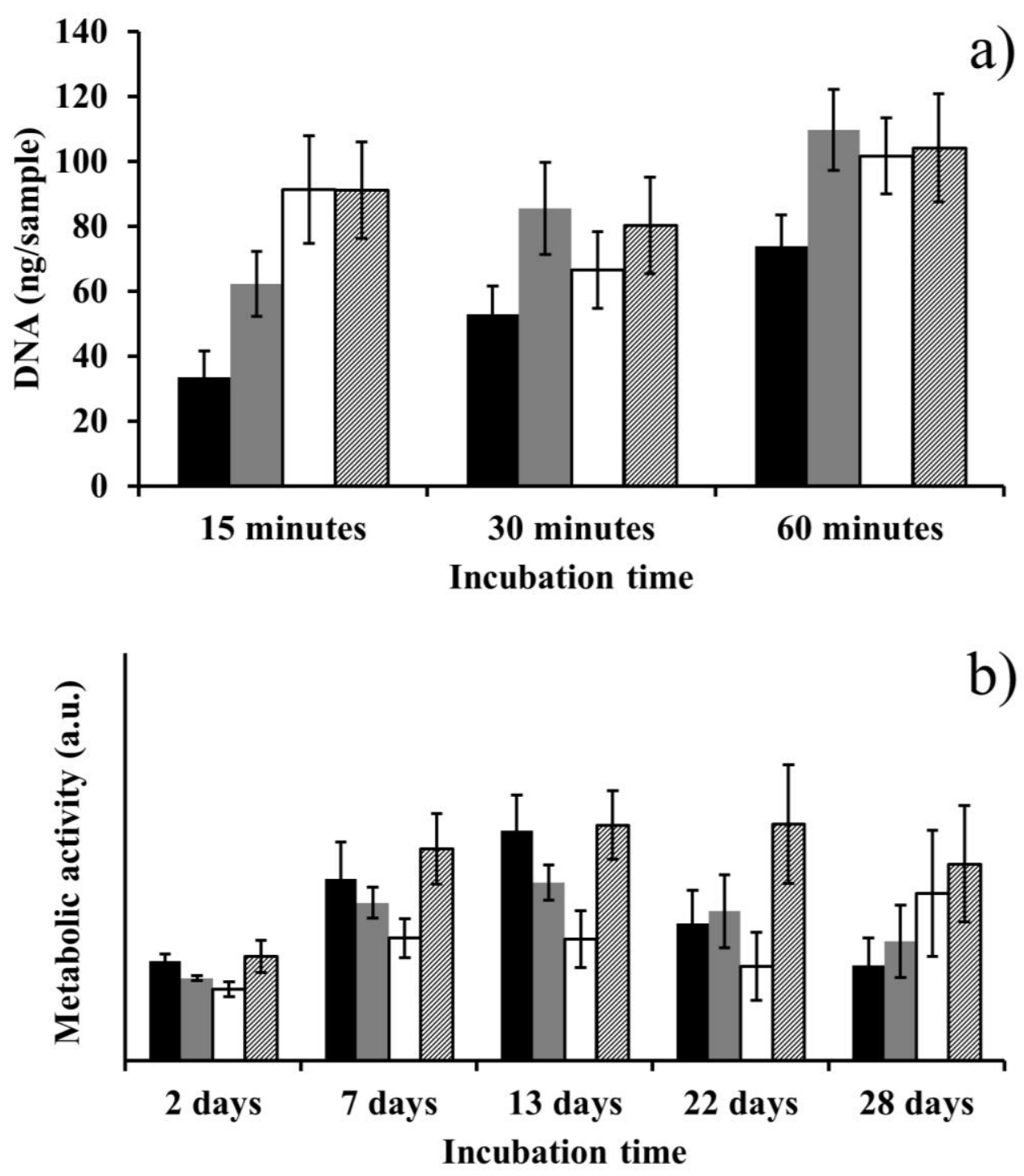

Figure 3. Cell adhesion expressed in DNA content of adhering cells per sample (a) and the evolution of the metabolic activity of hBMSCs over time (b) on porous composite films with 27\% (black bars), $52 \%$ (grey bars) and $71 \%$ (white bars) porosity and TCPS (bars with black shading). 
Good adhesion and proliferation of the hBMSCs on all composites was furthermore shown by Live/Dead ${ }^{\circledR}$ staining and SEM images. Figure $4 \mathrm{a}, \mathrm{b}$ and $\mathrm{c}$ show the Live/Dead® staining of cells after one day of culture. On all porous composites the cells were mainly alive and only a few dead cells were observed. After 28 days of culture all porous composites were covered in live cells and few dead cells as shown in figure $4 \mathrm{~d}$, e and f. This was confirmed by SEM images of the cell-covered films after 28 days of culture, which are shown in figure $4 \mathrm{~g}, \mathrm{~h}$ and $\mathrm{i}$. In high magnification SEM images in figure $4 \mathrm{j}, \mathrm{k}$ and 1 , calcium phosphate deposits can be observed which are evident of the osteogenic differentiation of the hBMSCs[27, 36].
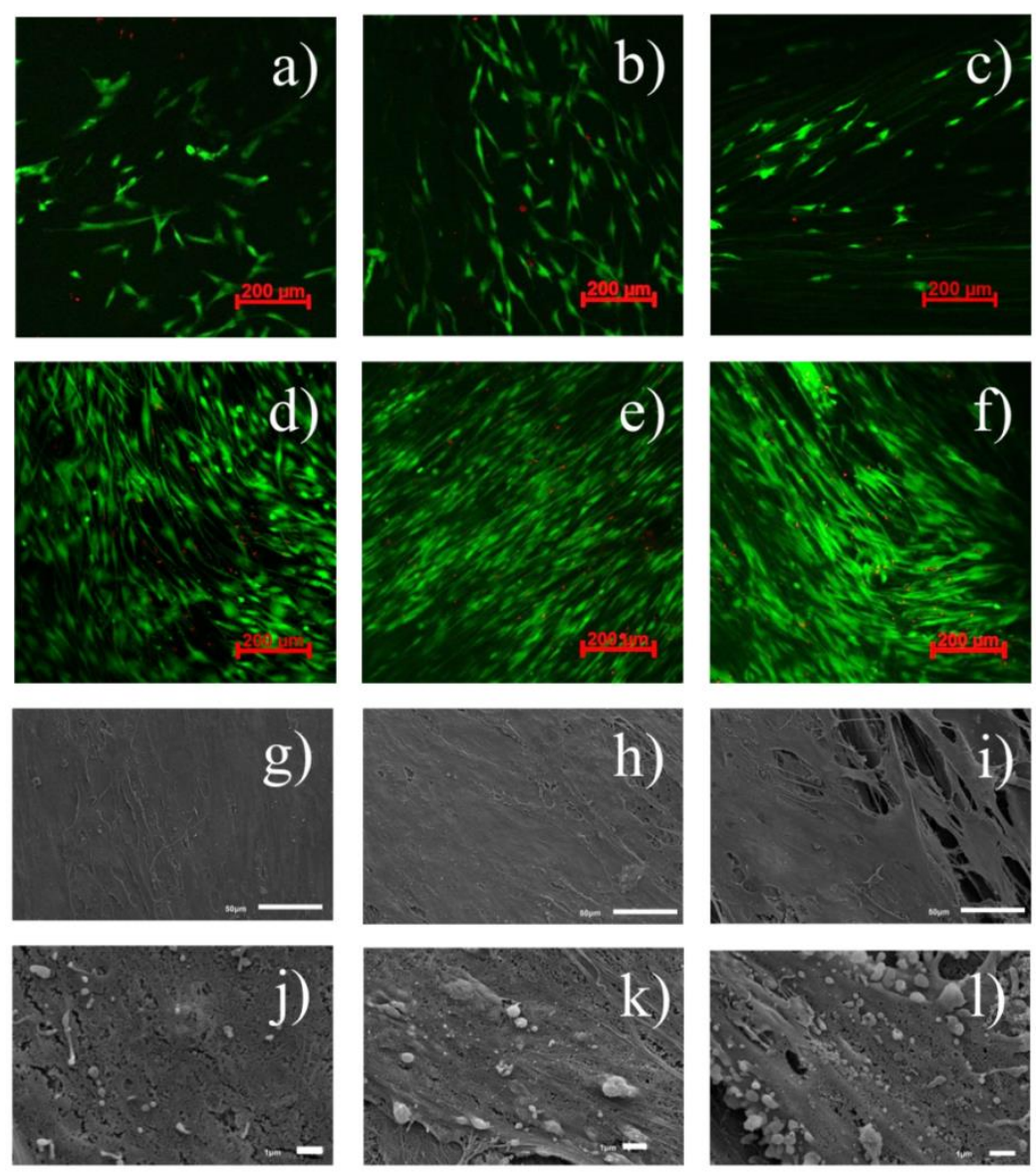

Figure 4. Live/Dead ${ }^{\circledR}$ images after 1 day $(a, b$ and $c)$ and 28 days ( $d$, $e$ and $\left.f\right)$ of culture, cell-covered composite film surfaces $(g, h$ and $i)$ imaged by SEM after 28 days of culture and high magnification SEM images of the cell surface after 28 days of culture ( $j, k$ and $l$ ) on composites with $27 \%(a, d, g$ and $j), 52 \%(b, e, h$ and $k$ ) and $71 \%$ (c, $f, i$ and l) porosity. Scale bars $g, h, i=50 \mu \mathrm{m}$; scale bars $j, k, l=1 \mu \mathrm{m}$. All images depicted are from cells of a single donor. 
Two distinguishing observations were made for the cells cultured on composites with $71 \%$ porosity. In the Live/Dead® staining images after 1 day of culture it was seen that the cells tended to align in the direction of the pore channels on these composites. To a lesser extent this was also seen on the composites with $52 \%$ porosity. Additionally, in the SEM images after 28 days of culture it was observed that cells were unable to traverse some of the large pore channels present on the surface of the composites with $71 \%$ porosity. This may have a profound effect on the differentiation of the hBMSCs on this composite as it can affect the cell morphology, which plays a pivotal role in cell differentiation[7, 13, 14].

To investigate the extent of osteogenic differentiation of the hBMSCs on the porous composites, the ALP activity and calcium production of the cells was quantified. Both assessments are depicted in figure 5 .
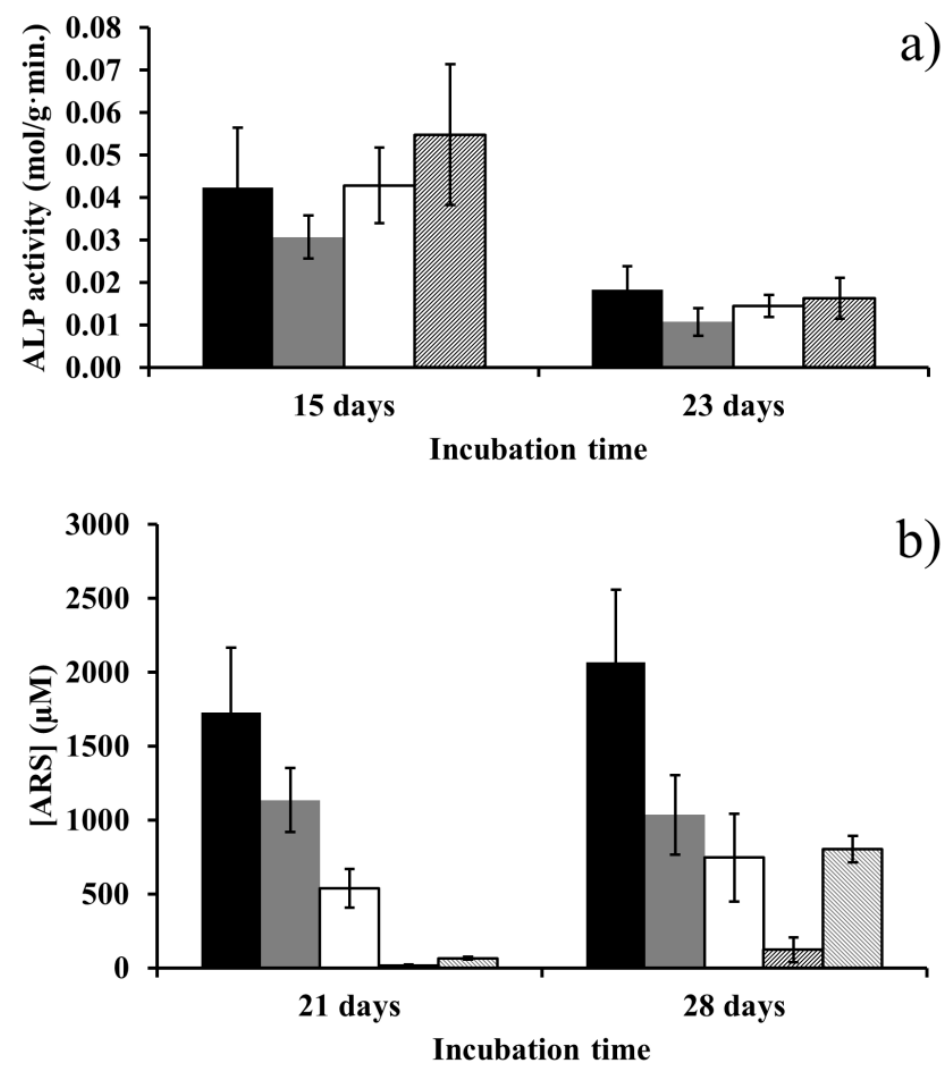

Figure 5. Quantification of the ALP activity of cells on porous composites (a) and ARS concentration on composites (b) with 27\% (black bars), 52\% (grey bars) and $71 \%$ (white bars) porosity, TCPS (bars with black shading) and Thermanox ${ }^{\mathrm{TM}}$ (bars with grey shading). 
The ALP activity shown in figure 5a is similar on all sample types and between 15 and 23 days of culture, the ALP activity on all samples including the TCPS control decreased. This is typical for the differentiation of cells into the osteogenic lineage for which ALP activity generally peaks and subsequently declines as the differentiated cells start to produce a mineralized extracellular matrix[37]. Interestingly, the decrease of the ALP activity coincides with the observed decrease of the metabolic activity between 13 and 22 days of culture (figure 3b) which can be related to the lower metabolic activity of cells in the osteogenic lineage compared to hBMSCs[35]. Since the ALP activity of cells can peak at different time points on the various porous composites we are not able to directly relate it to differences in the osteogenic differentiation of the cells[5, 37]. For this purpose, the ALP activity needs to be determined over a broader range of time points.

The ARS staining quantification given in figure $5 \mathrm{~b}$, shows that the cells produce significant amounts of calcium on the porous composites compared to the TCPS and Thermanox ${ }^{\mathrm{TM}}$ controls. The amount of calcium produced decreased with an increasing porosity and between 21 and 28 days the amount of additional calcium produced by cells on the porous composites is relatively low. On day 28 of culture, the amount of calcium produced by the cells is similar to the Thermanox ${ }^{\mathrm{TM}}$ control on composites with $52 \%$ and $71 \%$ porosity. On composites with $27 \%$ porosity, the calcium production of the cells is significantly higher. The enhanced calcium production on the $27 \%$ porosity composites compared to those with higher porosity indicates the strong maturation of the hBMSCs into the osteogenic lineage on the lower porosity composites.

A reduced differentiation potential of hBMSCs on composites with pores in a size range of 1 to $50 \mu \mathrm{m}$ and an increasing porosity has been described in literature before [2, 19]. It is thought that this is caused by an enhanced cell proliferation and decreased aggregation on materials with increasing porosity $[2,18,19,38]$.

Finally, the aligning of the cells with the pore channels of the higher porosity composites in this work and their inability to traverse large pore channels indicates that the adaptation of a favourable morphology for osteogenic differentiation may be hindered on these surfaces. The reduced stiffness of the composites with increasing porosity may further contribute to the weaker differentiation of hBMSCs on the higher porosity composites.

In previous works it has been shown that although an increasing porosity reduces osteogenic differentiation in vitro, it is beneficial for bone restoration in vivo[18, 19, 39]. Therefore, in vivo experiments are required to understand the effect of increasing porosity in photo-crosslinked PTMC and nHA composites on bone restoration efficacy. Currently we have already been able to prepare three-dimensional implant structures with resins described here and a low temperature extrusion-based additive manufacturing technique. A description of these structures is given in the supplementary information of this work.

\section{Conclusions}

In this work we have shown that resins based on PTMC-MA, nHA and EC are suitable for the preparation of porous composites with varying porosity. The resin viscosity and phase 
separation temperatures can be adjusted by controlling the EC concentration. Using this phase separation and photo-crosslinking, the EC concentration can directly affect the porosity, pore size and mechanical properties of the composites. An increasing porosity affected the surface morphology and reduced the stiffness of the porous composites. These modifications reduce the ability of hBMSCs to differentiate into the osteogenic lineage as was shown by the calcium deposition of the cells. This is in line with what has been reported in literature before on the in vitro differentiation of hBMSCs on porous materials. Reduced cell aggregation, ability to adapt a favourable morphology for osteogenic differentiation and stiffness of the porous composite may be the cause for this effect. Implantation experiments are required to assess the effect of porosity in porous, photocrosslinked PTMC-MA/nHA structures on the regeneration of bony defects.

\section{Acknowledgements}

The authors acknowledge the funding by the EU FP7 RAPIDOS project (grant no. NMP3SL-2013-604517) and the financial support of the Stiftung für Innovation, Entwicklung und Forschung Graubünden. Additionally, the authors thank Kuros BioSciences BV for their kind contributions of nano-hydroxyapatite.

\section{References}

1. Akin, F.A., H. Zreiqat, S. Jordan, M.B. Wijesundara, and L. Hanley, Preparation and analysis of macroporous TiO2 films on Ti surfaces for bone-tissue implants. $\mathbf{J}$ Biomed Mater Res, 2001. 57(4): p. 588-596.

2. Takahashi, Y. and Y. Tabata, Effect of the fiber diameter and porosity of nonwoven PET fabrics on the osteogenic differentiation of mesenchymal stem cells. $\mathrm{J}$ Biomater Sci Polym Ed, 2004. 15(1): p. 41-57.

3. Akay, G., M.A. Birch, and M.A. Bokhari, Microcellular polyHIPE polymer supports osteoblast growth and bone formation in vitro. Biomaterials, 2004. 25(18): p. 3991-4000.

4. Murphy, C.M., M.G. Haugh, and F.J. O'Brien, The effect of mean pore size on cell attachment, proliferation and migration in collagen-glycosaminoglycan scaffolds for bone tissue engineering. Biomaterials, 2010. 31(3): p. 461-466.

5. Salerno, A., D. Guarnieri, M. Iannone, S. Zeppetelli, and P.A. Netti, Effect of micro- and macroporosity of bone tissue three-dimensional-poly(epsiloncaprolactone) scaffold on human mesenchymal stem cells invasion, proliferation, and differentiation in vitro. Tissue Eng Part A, 2010. 16(8): p. 2661-2673.

6. Gomes, M.E., H.L. Holtorf, R.L. Reis, and A.G. Mikos, Influence of the porosity of starch-based fiber mesh scaffolds on the proliferation and osteogenic differentiation of bone marrow stromal cells cultured in a flow perfusion bioreactor. Tissue Eng, 2006. 12(4): p. 801-809. 
7. Zhao, L., L. Liu, Z. Wu, Y. Zhang, and P.K. Chu, Effects of micropitted/nanotubular titania topographies on bone mesenchymal stem cell osteogenic differentiation. Biomaterials, 2012. 33(9): p. 2629-2641.

8. Gittens, R.A., T. McLachlan, R. Olivares-Navarrete, Y. Cai, S. Berner, R. Tannenbaum, Z. Schwartz, K.H. Sandhage, and B.D. Boyan, The effects of combined micron-/submicron-scale surface roughness and nanoscale features on cell proliferation and differentiation. Biomaterials, 2011. 32(13): p. 3395-3403.

9. Zhang, J., X. Luo, D. Barbieri, A.M. Barradas, J.D. de Bruijn, C.A. van Blitterswijk, and H. Yuan, The size of surface microstructures as an osteogenic factor in calcium phosphate ceramics. Acta Biomater, 2014. 10(7): p. 3254-3263.

10. Deligianni, D.D., N.D. Katsala, P.G. Koutsoukos, and Y.F. Missirlis, Effect of surface roughness of hydroxyapatite on human bone marrow cell adhesion, proliferation, differentiation and detachment strength. Biomaterials, 2001. 22(1): p. 87-96.

11. Wall, I., N. Donos, K. Carlqvist, F. Jones, and P. Brett, Modified titanium surfaces promote accelerated osteogenic differentiation of mesenchymal stromal cells in vitro. Bone, 2009. 45(1): p. 17-26.

12. Muller, P., U. Bulnheim, A. Diener, F. Luthen, M. Teller, E.D. Klinkenberg, H.G. Neumann, B. Nebe, A. Liebold, G. Steinhoff, and J. Rychly, Calcium phosphate surfaces promote osteogenic differentiation of mesenchymal stem cells. Journal of Cellular and Molecular Medicine, 2008. 12(1): p. 281-291.

13. Xia, L., K. Lin, X. Jiang, B. Fang, Y. Xu, J. Liu, D. Zeng, M. Zhang, X. Zhang, J. Chang, and Z. Zhang, Effect of nano-structured bioceramic surface on osteogenic differentiation of adipose derived stem cells. Biomaterials, 2014. 35(30): p. 85148527.

14. Hulshof, F.F.B., B. Papenburg, A. Vasilevich, M. Hulsman, Y. Zhao, M. Levers, N. Fekete, M. de Boer, H. Yuan, S. Singh, N. Beijer, M.A. Bray, D.J. Logan, M. Reinders, A.E. Carpenter, C. van Blitterswijk, D. Stamatialis, and J. de Boer, Mining for osteogenic surface topographies: In silico design to in vivo osseointegration. Biomaterials, 2017. 137: p. 49-60.

15. Chatterjee, K., S. Lin-Gibson, W.E. Wallace, S.H. Parekh, Y.J. Lee, M.T. Cicerone, M.F. Young, and C.G. Simon, Jr., The effect of 3D hydrogel scaffold modulus on osteoblast differentiation and mineralization revealed by combinatorial screening. Biomaterials, 2010. 31(19): p. 5051-5062.

16. Wen, J.H., L.G. Vincent, A. Fuhrmann, Y.S. Choi, K.C. Hribar, H. TaylorWeiner, S. Chen, and A.J. Engler, Interplay of matrix stiffness and protein tethering in stem cell differentiation. Nature Materials, 2014. 13(10): p. 979-987.

17. Nam, J., J. Johnson, J.J. Lannutti, and S. Agarwal, Modulation of embryonic mesenchymal progenitor cell differentiation via control over pure mechanical modulus in electrospun nanofibers. Acta Biomater, 2011. 7(4): p. 1516-1524. 
18. Hutmacher, D.W., J.T. Schantz, C.X. Lam, K.C. Tan, and T.C. Lim, State of the art and future directions of scaffold-based bone engineering from a biomaterials perspective. Journal of Tissue Engineering and Regenerative Medicine, 2007. 1(4): p. 245-260.

19. Karageorgiou, V. and D. Kaplan, Porosity of $3 D$ biomaterial scaffolds and osteogenesis. Biomaterials, 2005. 26(27): p. 5474-5491.

20. Kuboki, Y., Q. Jin, and H. Takita, Geometry of carriers controlling phenotypic expression in BMP-induced osteogenesis and chondrogenesis. Journal of Bone and Joint Surgery American Volume, 2001. 83-A Suppl 1(Pt 2): p. S105-S115.

21. Kuboki, Y., Q. Jin, M. Kikuchi, J. Mamood, and H. Takita, Geometry of artificial ECM: sizes of pores controlling phenotype expression in BMP-induced osteogenesis and chondrogenesis. Connective Tissue Research, 2002. 43(2-3): p. 529-534.

22. Bose, S., M. Roy, and A. Bandyopadhyay, Recent advances in bone tissue engineering scaffolds. Trends in Biotechnology, 2012. 30(10): p. 546-554.

23. Tarafder, S., V.K. Balla, N.M. Davies, A. Bandyopadhyay, and S. Bose, Microwave-sintered 3D printed tricalcium phosphate scaffolds for bone tissue engineering. Journal of Tissue Engineering and Regenerative Medicine, 2013. 7(8): p. 631-641.

24. Habibovic, P. and K. de Groot, Osteoinductive biomaterials--properties and relevance in bone repair. Journal of Tissue Engineering and Regenerative Medicine, 2007. 1(1): p. 25-32.

25. Engler, A.J., S. Sen, H.L. Sweeney, and D.E. Discher, Matrix elasticity directs stem cell lineage specification. Cell, 2006. 126(4): p. 677-689.

26. Chapter 6 of this thesis; Guillaume, O., M.A. Geven, D.W. Grijpma, T.T. Tang, L. Qin, Y.X. Lai, H. Yuan, R.G. Richards, and D. Eglin, Poly(trimethylene carbonate) and nano-hydroxyapatite porous scaffolds manufactured by stereolithography. Polymers for Advanced Technologies, 2017. 28(10): p. 12191225.

27. Chapter 7 of this thesis; Guillaume, O., M.A. Geven, C.M. Sprecher, V.A. Stadelmann, D.W. Grijpma, T.T. Tang, L. Qin, Y. Lai, M. Alini, J.D. de Bruijn, H. Yuan, R.G. Richards, and D. Eglin, Surface-enrichment with hydroxyapatite nanoparticles in stereolithography-fabricated composite polymer scaffolds promotes bone repair. Acta Biomater, 2017. 54: p. 386-398.

28. LeGeros, R.Z., Calcium phosphate-based osteoinductive materials. Chemical Reviews, 2008. 108(11): p. 4742-4753.

29. Barbieri, D., A.J. Renard, J.D. de Bruijn, and H. Yuan, Heterotopic bone formation by nano-apatite containing poly(D,L-lactide) composites. European Cells and Materials, 2010. 19: p. 252-261.

30. Chapter 4 of this thesis; Geven, M.A., V. Varjas, L. Kamer, X. Wang, J. Peng, D. Eglin, and D.W. Grijpma, Fabrication of patient specific composite orbital floor 
implants by stereolithography. Polymers for Advanced Technologies, 2015. 26(12): p. 1433-1438.

31. Chapter 3 of this thesis; Geven, M.A., D. Barbieri, H. Yuan, J.D. de Bruijn, and D.W. Grijpma, Preparation and mechanical properties of photo-crosslinked poly(trimethylene carbonate) and nano-hydroxyapatite composites. Clinical Hemorheology and Microcirculation, 2015. 60(1): p. 3-11.

32. Gadelmawla, E.S., M.M. Koura, T.M.A. Maksoud, I.M. Elewa, and H.H. Soliman, Roughness parameters. Journal of Materials Processing Technology, 2002. 123(1): p. 133-145.

33. Herrmann, M., J.J. Bara, C.M. Sprecher, U. Menzel, J.M. Jalowiec, R. Osinga, A. Scherberich, M. Alini, and S. Verrier, Pericyte plasticity - comparative investigation of the angiogenic and multilineage potential of pericytes from different human tissues. European Cells and Materials, 2016. 31: p. 236-249.

34. Zhang, R. and P.X. Ma, Poly(alpha-hydroxyl acids)/hydroxyapatite porous composites for bone-tissue engineering. I. Preparation and morphology. J Biomed Mater Res, 1999. 44(4): p. 446-455.

35. Westhrin, M., M. Xie, M.O. Olderoy, P. Sikorski, B.L. Strand, and T. Standal, Osteogenic differentiation of human mesenchymal stem cells in mineralized alginate matrices. PLoS One, 2015. 10(3): p. e0120374.

36. Martins, A., A.R. Duarte, S. Faria, A.P. Marques, R.L. Reis, and N.M. Neves, Osteogenic induction of hBMSCs by electrospun scaffolds with dexamethasone release functionality. Biomaterials, 2010. 31(22): p. 5875-5885.

37. Birmingham, E., G.L. Niebur, P.E. McHugh, G. Shaw, F.P. Barry, and L.M. McNamara, Osteogenic differentiation of mesenchymal stem cells is regulated by osteocyte and osteoblast cells in a simplified bone niche. European Cells and Materials, 2012. 23: p. 13-27.

38. Collins, J.M., P. Ayala, T.A. Desai, and B. Russell, Three-dimensional culture with stiff microstructures increases proliferation and slows osteogenic differentiation of human mesenchymal stem cells. Small, 2010. 6(3): p. 355-360.

39. Roy, T.D., J.L. Simon, J.L. Ricci, E.D. Rekow, V.P. Thompson, and J.R. Parsons, Performance of degradable composite bone repair products made via threedimensional fabrication techniques. Journal of Biomedical Materials Research A, 2003. 66a(2): p. 283-291. 


\section{Chapter 9 - Supplementary information}

Low temperature extrusion-based additive manufacturing using LowEC and MidEC resins as inks

In bone regeneration, implants with a multiscale porosity are deemed ideal. Large macropores of more than $300 \mu \mathrm{m}$ in diameter allow for bone ingrowth as well as vascularization which is essential for reconstruction[18-22]. Smaller pores $(\varnothing<20 \mu \mathrm{m})$ in the trabeculae of implants can improve osteogenicity[22-24] by increasing surface roughness of the implant, enhancing local concentration of proteins and improving nutritional supply and waste removal. Using the resins reported in the main article as inks for extrusion-based additive manufacturing, we are able to prepare such implants with a multiscale porosity.

Three dimensional structures with a macro- and micro-porosity were fabricated using a RegenHU 3D Discovery apparatus. LowEC and MidEC resins were used as inks and extruded from an air-pressurized and heated cartridge onto a cooled build plate. The build plate was perfused with cooled water of which the temperature was controlled using a thermostated water bath. To prevent the formation of a layer of condensation on top of the build plate a nitrogen flow was applied over it.

Hardware and conditions applied for additive manufacturing are given in table S1.

Table S1. Hardware and printing parameters applied for additive manufacturing.

\begin{tabular}{lcc}
\hline & \multicolumn{2}{c}{ Inks } \\
\cline { 2 - 3 } Hardware/parameter & LowEC & MidEC \\
\hline Tip shape $(-)$ & Conical & Straight \\
Tip material $(-)$ & Tapered plastic & Stainless steel \\
Inner diameter tip $(\mathrm{mm})$ & 0.58 & 0.33 \\
Tip length $(\mathrm{mm})$ & 30.5 & 6.35 \\
Cartridge temperature $\left({ }^{\circ} \mathrm{C}\right)$ & 50 & 50 \\
Build plate temperature $\left({ }^{\circ} \mathrm{C}\right)$ & $7^{\mathrm{a}}$ & $7^{\mathrm{a}}$ \\
Applied air pressure $(\mathrm{bar})$ & 4 & 3 \\
Movement rate $(\mathrm{mm} / \mathrm{s})^{\mathrm{b}}$ & 10 & 15 \\
Step height $(\mathrm{mm})$ & 0.43 & 0.26 \\
\hline
\end{tabular}

${ }^{\mathrm{a}}$ Minimum achievable build plate temperature.

${ }^{\mathrm{b}}$ Movement rate of the print head relative to the build plate.

Directly after extrusion-based additive manufacturing, composite structures were stored at $20{ }^{\circ} \mathrm{C}$ at least overnight. The structures were subsequently photo-crosslinked in a UVcrosslinking box at $365 \mathrm{~nm}$ and $11 \mathrm{~mW} / \mathrm{cm}^{2}$ under $\mathrm{N}_{2}$ atmosphere. Top and bottom of the structures were exposed to UV light for 30 minutes each at room temperature. The photocrosslinked structures were extracted and dried as described for porous composite films in the main article. 
Structures were visualized by micro-CT (Scanco Medical $\mu$ CT 40) and by SEM of freezefractured cross-sections (JEOL JSM-IT100 SEM). Pore widths were analysed as described in the main article.

In figure $\mathrm{S} 1$ an overview is given of micro-CT reconstructions and SEM images of the macro- and micro-porous composite structures prepared by additive manufacturing. In the $\mu \mathrm{CT}$ reconstructions the thickness of the struts of the structures was shown to be homogeneous with an average width of $0.82 \mathrm{~mm}$ and $0.66 \mathrm{~mm}$ for structures prepared with LowEC and MidEC inks. In SEM images, micro-pores in the struts of the fabricated structures were observed. This confirms phase separation occurring in the structure before photo-crosslinking. Micro-pores were also observed on the surface of the struts of the structures as seen in figure $\mathrm{S} 1 \mathrm{e}$ and $\mathrm{g}$. In figure $\mathrm{S} 1 \mathrm{j}$ and $\mathrm{l}$, nHA particles can be observed inbetween and inside the micro-pores in the cross-section of the structures. The micro-pore width in composite structures prepared with the LowEC ink ranged from 0.2 to $8.4 \mu \mathrm{m}$ with an average of $1.9 \mu \mathrm{m}$. Composite structures prepared with the MidEC ink possessed micropores with a width ranging from 0.4 to $10.7 \mu \mathrm{m}$ with an average of $3.2 \mu \mathrm{m}$.
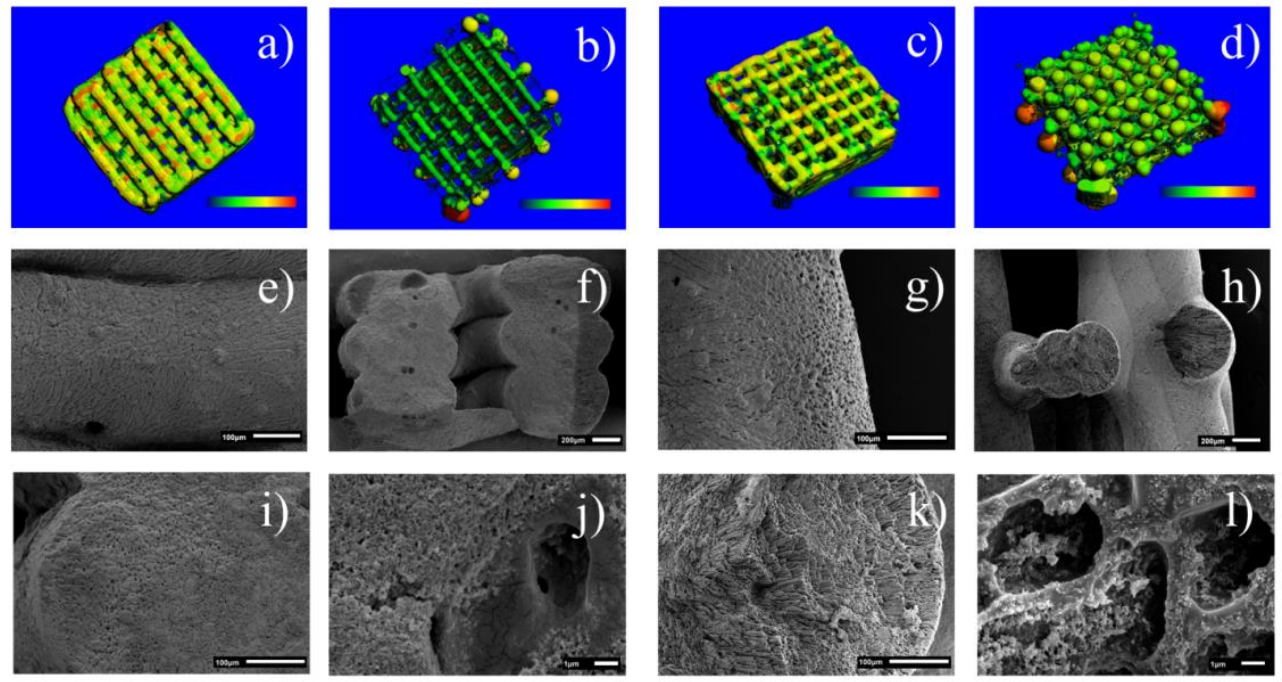

Figure S1. $\mu C T$ reconstructions of the trabecular space $(a$ and $c$ ) and pore space ( $b$ and $d$ ) of fabricated structures and SEM images of the cross-sections (e to l) after freeze-fracturing of structures fabricated by LowEC ink ( $a, b, e, f, i$ and $j)$ and MidEC ink ( $c, d, g, h, k$ and $l)$. Heat maps $a, c$ : from 0 to $1.1 \mathrm{~mm}$; heat maps $b, d:$ from 0 to $1.7 \mathrm{~mm}$. Scale bars $e, i, g, k=100 \mu \mathrm{m}$; scale bars $f, h=200 \mu \mathrm{m}$; scale bars $j, l=1 \mu \mathrm{m}$. 


\section{Appendix A - Tensile properties of photo-crosslinked poly(trimethylene carbonate-co-e-caprolactone) and nano- hydroxyapatite composites}

\section{Introduction}

In the restoration of large bone defects, degradable implants are ideally used. These implants should guide or induce bone growth in the defect. To avoid problems such as stress shielding of de novo formed bone, a lasting foreign body response or premature failure, the degradation should be well-controlled. In this thesis, as well as in previous work from our group, it was demonstrated that photo-crosslinked networks of methacrylate endgroup functionalized poly(trimethylene carbonate) (PTMC) degrade very slowly when the starting PTMC macromer is of low molecular weight $(<20 \mathrm{~kg} / \mathrm{mol})$. Copolymerization of trimethylene carbonate with other, ester-containing monomers yields copolymers that can form networks with a higher rate of degradation. Here we have prepared copolymer networks by photo-crosslinking of methacrylate end-group functionalized poly(trimethylene carbonate-co- $\varepsilon$-caprolactone) (PTMCECL) of low molecular weight $(10.3 \mathrm{~kg} / \mathrm{mol})$. Initial mechanical characterization of these networks and of their composites with nano-hydroxyapatite (nHA) was done by tensile testing and the results were compared to those of analogous PTMC networks described in Chapter $\mathbf{3}$ of this thesis.

\section{Experimental}

Synthesis of three-armed PTMCECL oligomer was performed by ring-opening polymerization of trimethylene carbonate and $\varepsilon$-caprolactone. The $\varepsilon$-caprolactone was dried over calcium hydride and distilled under vacuum after which both monomers $(0.49 \mathrm{~mol}$ each) were charged into a three-neck round-bottomed flask under $\mathrm{N}_{2}$ atmosphere. 1,1,1Tris(hydroxymethyl)propane $(11 \mathrm{mmol})$ and tin(II) ethylhexanoate $(0.13 \mathrm{wt} . \%)$ were added as initiator and catalyst respectively. The polymerization reaction was then conducted under $\mathrm{N}_{2}$ atmosphere at $130{ }^{\circ} \mathrm{C}$ for three days. The resulting oligomer was dissolved in dry dichloromethane and subsequently hydroquinone $(0.1 \mathrm{wt} . \%)$, triethylamine $(3 \mathrm{~mol} / \mathrm{mol}$ of PTMCeCL hydroxyl end-groups) and methacrylic anhydride $(3 \mathrm{~mol} / \mathrm{mol}$ of PTMCeCL hydroxyl end-groups) were added under stirring. This functionalization reaction was performed under $\mathrm{N}_{2}$ atmosphere for 5 days at room temperature in the dark. The resulting PTMCECL macromer was purified and recovered by precipitation of the reaction mixture in cold methanol and subsequent extraction in additional cold methanol. The macromer was dried in vacuo at room temperature until constant weight.

The ratio of trimethylene carbonate repeat units to $\varepsilon$-caprolactone repeat units, number average molecular weight and degree of functionalization of the PTMCECL macromer was determined by ${ }^{1} \mathrm{H}-\mathrm{NMR}$ (Bruker Ascend 400/Avance III $400 \mathrm{MHz}$ NMR spectrometer) in deuterated chloroform. 
Photo-crosslinked films of PTMCECL macromer with various nHA contents were prepared as described for photo-crosslinked PTMC films in Chapter $\mathbf{3}$ of this thesis. Photocrosslinked PTMCECL films with intended nHA contents of 0, 10, 20 and 35 wt.\% were prepared. The thickness of the films was between 250 and $350 \mu \mathrm{m}$.

Tensile mechanical properties were determined using a Zwick Z020 tensile tester equipped with a 500-N load cell. The initial grip-to-grip separation was $50 \mathrm{~mm}$, and the crosshead speed was $50 \mathrm{~mm} / \mathrm{min}$. Sample dimensions were $100 \times 5 \mathrm{~mm}^{2}$.

\section{Results and discussion}

By ${ }^{1} \mathrm{H}$-NMR it was determined that the ratio of trimethylene carbonate to $\varepsilon$-caprolactone repeat units in PTMC\&CL macromers was 48:52. A number average molecular weight of $10.3 \mathrm{~kg} / \mathrm{mol}$ and a degree of functionalization of $>99 \%$ was also determined by ${ }^{1} \mathrm{H}-\mathrm{NMR}$.

In table 1 an overview is given of the tensile properties determined for photo-crosslinked PTMC $\varepsilon$ CL and its composites. The tensile properties of photo-crosslinked PTMC prepared from macromers with a molecular weight of $10.4 \mathrm{~g} / \mathrm{mol}$ and its composites (Chapter 3) are also given as a comparison.

Table 1. Tensile properties of photo-crosslinked PTMC and PTMCECL and their composites with nHA.

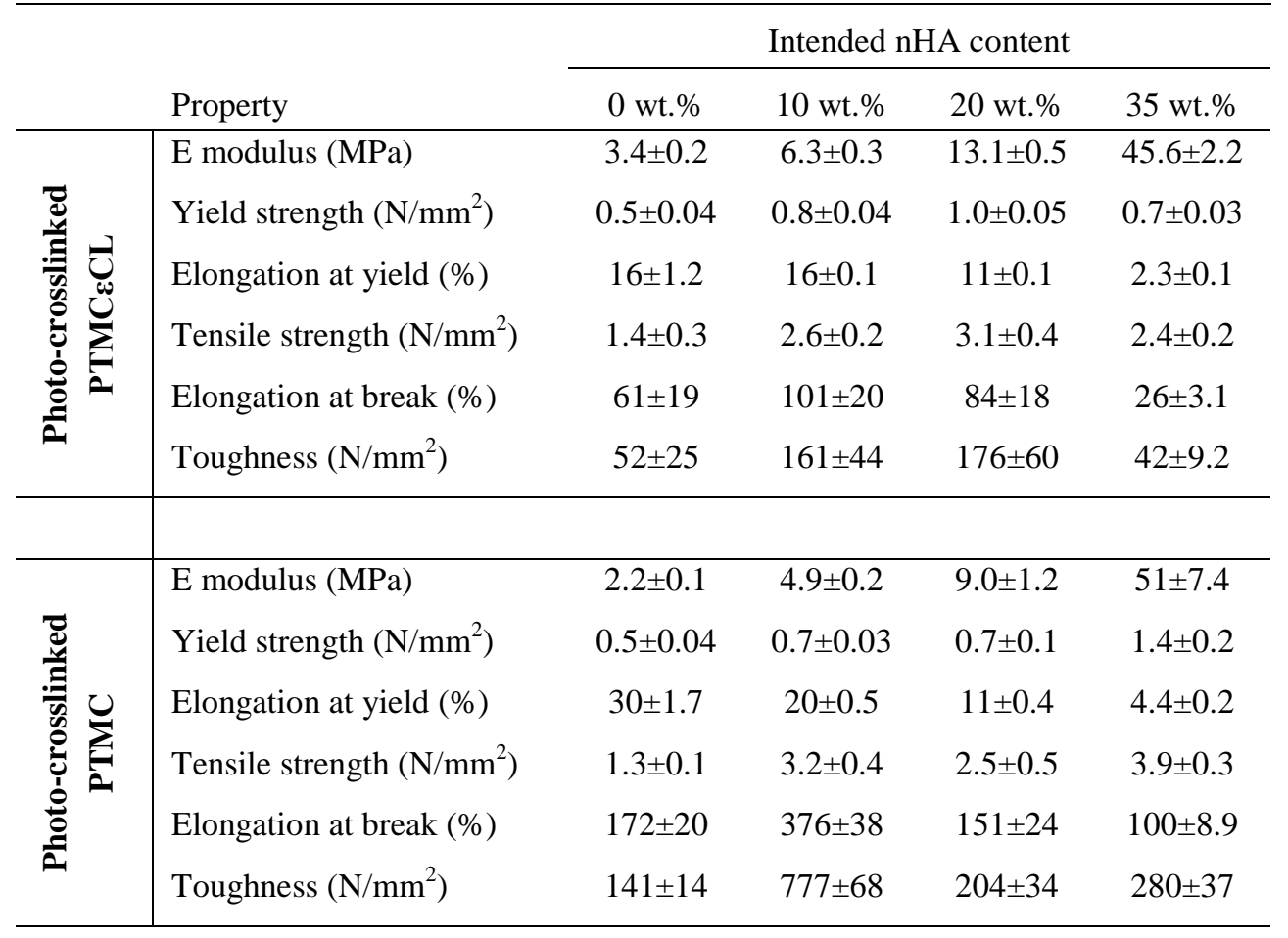

The tensile data shows that photo-crosslinked PTMCeCL is similarly affected by nHA incorporation as photo-crosslinked PTMC. In both cases, the stiffness of the films increases 
with increasing nHA content. Also for photo-crosslinked PTMCeCL composites, an optimum in strain at break and toughness is observed at intermediate nHA contents, although less pronounced than for photo-crosslinked PTMC composites. Clear differences between the composites can also be observed. At nHA contents below 35 wt. $\%$, the stiffness of the photo-crosslinked PTMCECL and its composites is higher than that of the photo-crosslinked PTMC and its composites. Photo-crosslinked PTMCeCL and its composites had significantly lower strains at break and toughness than the photocrosslinked PTMC and its composites. This is also illustrated in figure 1, where the lower strain at break of the photo-crosslinked PTMCeCL and its composites can clearly be observed.

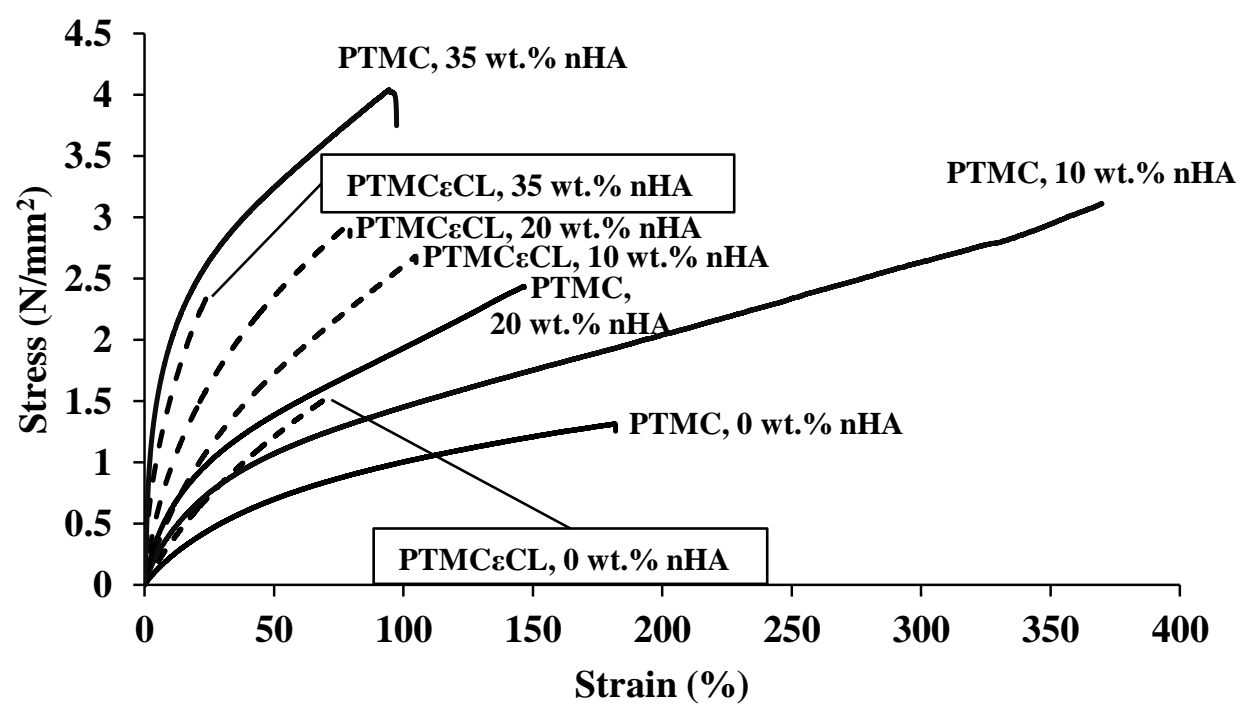

Figure 1. Stress-strain curves of photo-crosslinked PTMC (solid curves) and PTMCECL (dashed curves) and their composites with nHA.

\section{Conclusions}

Photo-crosslinked PTMCECL and its composites with nHA were characterized by tensile testing and compared with analogous photo-crosslinked PTMC composites. The results showed that photo-crosslinked PTMCeCL and its composites were more rigid but less tough than photo-crosslinked PTMC and its composites. 


\section{Appendix B - Icariin-releasing formulations for applications in bone restoration}

\section{Introduction}

Restoration of bone defects by synthetic composite implants is an interesting alternative for the use of natural grafts. To date, synthetic implant materials do not yet match the bone regenerative capacity of natural grafts. One of the reasons is that natural grafts do not possess the osteogenicity of natural graft materials. This may be imbued by pre-seeding of an implant by osteoprogenitor cells prior to implantation. Alternatively, osteopromotive proteins or medicines may be loaded in or onto the implant. Icariin is one such osteopromotive medicine. It is a plant-derived phytomolecule from epimedium species and has been shown to be effective in enhancing bone formation and reducing osteoporosis in animal studies. Here we investigate two controlled release formulations for icariin, $\operatorname{poly}(\varepsilon-$ caprolactone) (PCL) microspheres loaded with icariin using a solid-in-oil-in-water emulsion and composite structures prepared from photo-crosslinked poly(trimethylene carbonate) and nHA loaded with icariin by dip-coating.

\section{Experimental}

Hydroxyl group-terminated PCL oligomers were prepared by ring-opening polymerization of $\varepsilon$-caprolactone which was dried over calcium hydride and distilled under vacuum. Under $\mathrm{N}_{2}$ atmosphere, $0.44 \mathrm{~mol}$ of $\varepsilon$-caprolactone monomer, 1,6-hexanediol initiator $(5.1 \mathrm{mmol})$ and tin(II) ethylhexanoate catalyst $(0.13 \mathrm{wt} . \%)$ were mixed. The polymerization reaction was subsequently performed at $130{ }^{\circ} \mathrm{C}$ for three days. The resulting oligomer was recovered by dissolution in dichloromethane and subsequent precipitation in cold methanol. The precipitate was dried in vacuo at room temperature until constant weight.

The number average molecular weight of the PCL was determined by ${ }^{1} \mathrm{H}-\mathrm{NMR}$ (Bruker Ascend 400/Avance III $400 \mathrm{MHz}$ NMR spectrometer) in deuterated chloroform. A value of $11 \mathrm{~kg} / \mathrm{mol}$ was determined.

Icariin-loaded microspheres were prepared by a solid-in-oil-in-water emulsion. Icariin was initially dispersed in $5 \mathrm{ml}$ of PCL solution in dichloromethane by ball-milling. The icariin concentration was $1 \mathrm{wt}$ \% relative to the PCL mass. The resulting dispersion was added dropwise to a mechanically stirred solution of poly(vinyl alcohol) (PVA, 9-10 kg/mol, 80\% hydrolyzed) $(250 \mathrm{ml})$. The resulting solid-in-oil-in-water emulsion was stirred overnight to evaporate the dichloromethane and harden the microspheres. These were subsequently recovered by filtration (Whatman, grade 597 filter paper) and thereafter washed with ultrapure MilliQ water. The washed microspheres were dried by lyophilization over three days. The effect of the PCL concentration, the PVA concentration and the stirring rate used during preparation of microspheres on microsphere size, drug loading and -release was 
investigated. Table 1 gives an overview of the concentrations and stirring rates that were used.

Table 1. Overview of PCL concentration, PVA concentration and stirring rate applied during preparation of icariin-loaded microspheres.

\begin{tabular}{lccc}
\hline Adjusted variable & {$[\mathrm{PCL}](\% \mathrm{w} / \mathrm{v})$} & {$[\mathrm{PVA}](\% \mathrm{w} / \mathrm{v})$} & Stirring rate $(\mathrm{rpm})$ \\
\hline & 10 & & \\
PCL concentration & 20 & 4 & 800 \\
& 30 & & \\
& 40 & 1 & 800 \\
PVA concentration & 20 & 2 & \\
& & 4 & 400 \\
& & 6 & 800 \\
Stirring rate & 20 & 4 & 1000 \\
& & & 1200 \\
\hline
\end{tabular}

Microsphere size was determined from SEM images (YL30 ESEM-FEG Philips) using Image J analysis.

Photo-crosslinked poly(trimethylene carbonate) and nHA composite structures were prepared as described in Chapter 6 of this thesis, using stereolithography and the same scaffold design and resin formulations, and loaded with icariin by dip-coating. Structures with 0, 24 and 47 wt.\% nano-hydroxyapatite (nHA) were prepared. To assess the effect of nHA content on icariin loading and release, each structure was immersed in $1.39 \mathrm{mg} / \mathrm{ml}$ icariin solution in methanol overnight and thereafter dried under ambient conditions. Furthermore, structures with 47 wt.\% nHA were dip-coated in icariin solutions in methanol with different concentrations of icariin to assess the effect of the icariin concentration on drug loading.

The loading of the microspheres and structures with icariin was determined by immersing them in methanol $(\mathrm{n}=3)$. Approximately $2.5 \mathrm{mg}$ of microspheres was immersed per milliliter of methanol and each structure was immersed in $13 \mathrm{ml}$ of methanol. Microspheres and structures were extracted in methanol for three days while shaking. The concentration of icariin in methanol was determined by UV-Vis spectroscopy (Agilent Technologies, Cary 300) at $270 \mathrm{~nm}$ and used to calculate the total mass of icariin extracted $\left(\mathrm{m}_{\mathrm{ic}}\right)$. For microspheres, the drug loading ( $\mathrm{L}$ in $\mathrm{mg} / \mathrm{g}$ ) was determined according to equation 1 , where $\mathrm{m}_{\mathrm{us}}$ is the microsphere mass used during icariin extraction. Drug loading on structures was directly defined as the total mass of icariin extracted per structure, since they are of similar size and porosity (Chapter 7 of this thesis). 


$$
L=\frac{m_{i c}}{m_{\mu s}}
$$

The encapsulation efficiency ( $\mathrm{E}$ in $\%$ ) of icariin in the microspheres was additionally determined according to equation 2 , where $\mathrm{L}_{\mathrm{t}}$ is the maximum possible drug loading. The latter is defined as the initial amount of icariin relative to PCL used during microsphere preparation $(10 \mathrm{mg} / \mathrm{g})$.

$$
E=\frac{L}{L_{t}} * 100 \%
$$

Icariin-loaded microspheres as well as structures were immersed in phosphate buffered saline supplemented with $0.5 \%(\mathrm{w} / \mathrm{v})$ Tween $80 \AA(\mathrm{PBST})$ to investigate drug release. Approximately $25 \mathrm{mg}$ of microspheres was immersed in $2.5 \mathrm{ml}$ of PBST and each structure in $3.5 \mathrm{ml}$ PBST. All samples were thereafter kept at $37^{\circ} \mathrm{C}$ while shaking. At selected time points, all but $0.5 \mathrm{ml}$ of the PBST was replaced with fresh PBST. The collected PBST was analyzed by UV-Vis spectroscopy at $270 \mathrm{~nm}$ to determine the concentration of icariin. Cumulative release profiles were constructed accordingly.

\section{Results and discussion}

Icariin-loaded PCL microspheres were prepared by a solid-in-oil-in-water emulsion technique using PVA as a microsphere stabilizer. The formed microspheres were generally rounded and smooth, as is exemplified in figure 1.
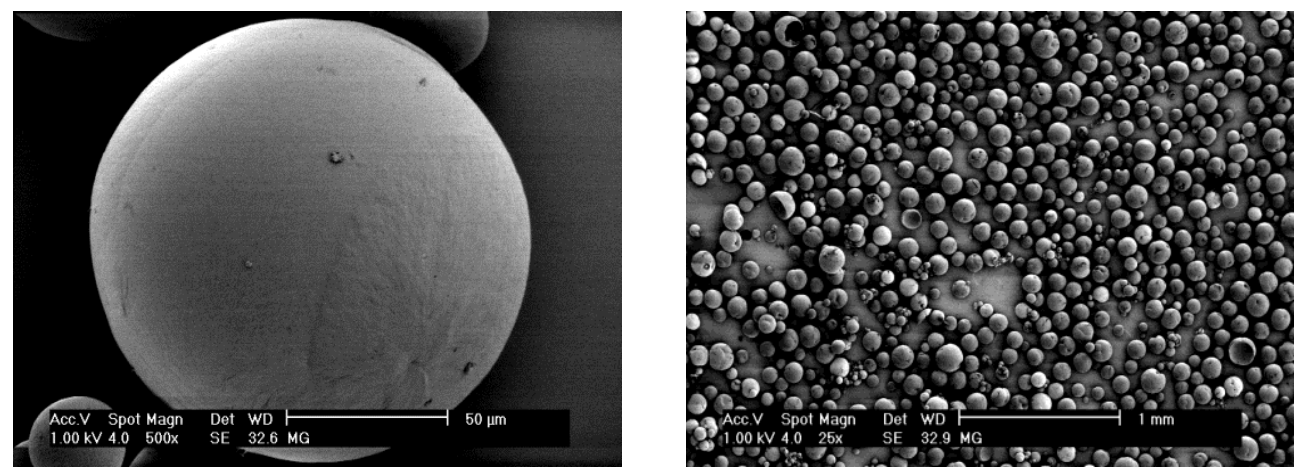

Figure 1. SEM images of icariin-loaded PCL microspheres prepared with $20 \%(w / v) P C L, 4 \%(w / v) P V A$ and at a stirring rate of 800 rpm at high (left image) and low (right image) magnification.

The effect of PCL concentration, PVA concentration and stirring rate used during microsphere preparation was investigated. Figure 2 depicts the effect of these parameters on microsphere diameter, icariin loading and encapsulation efficiency. 


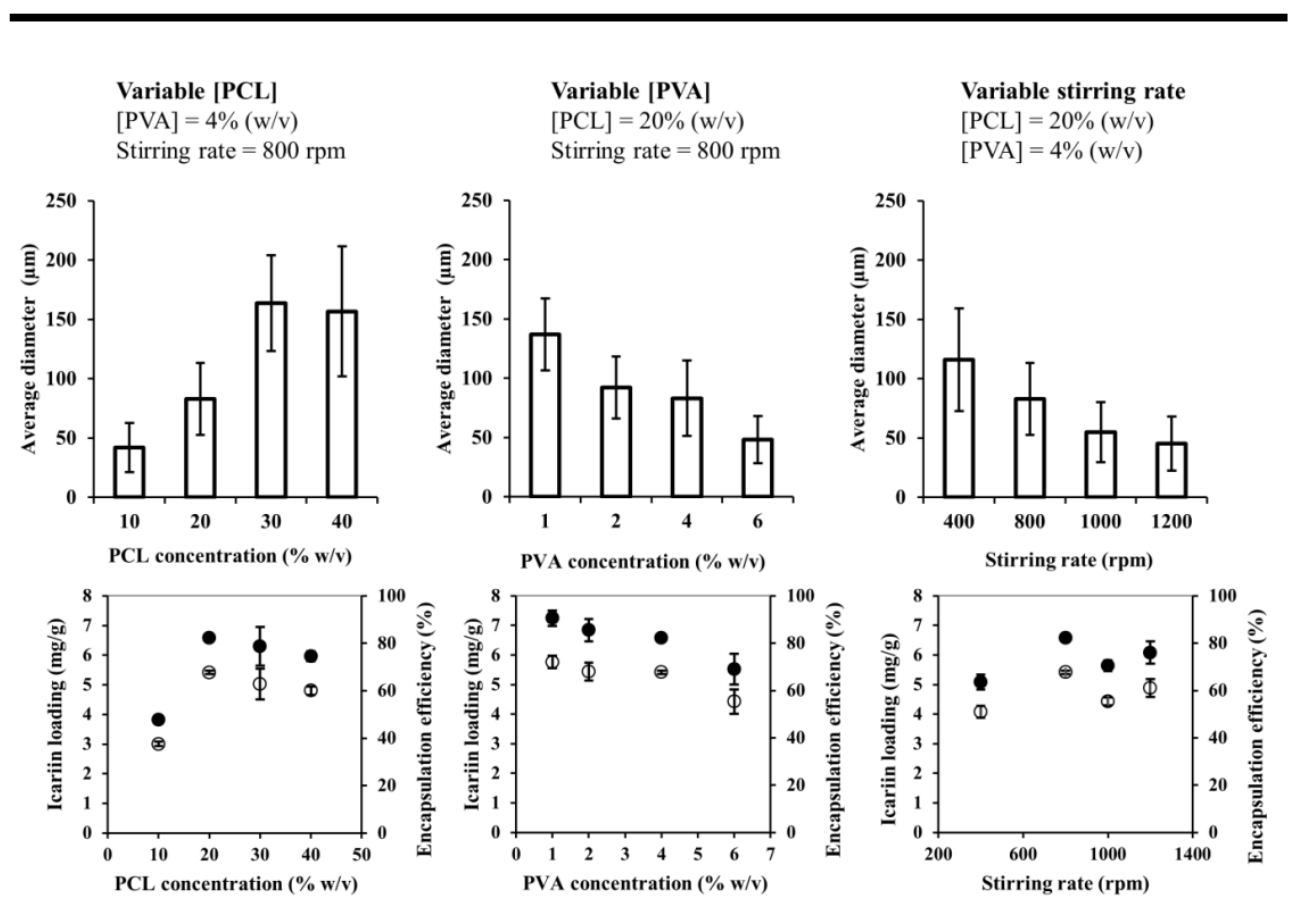

Figure 2. Average diameter (top row of graphs) and icariin loading (closed symbols) and encapsulation efficiency (open symbols) (bottom row of graphs) of PCL microspheres. Error bars in the top row of figures indicate the minimum and maximum particle sizes measured.

Figure 2 shows that the diameter of the icariin-loaded PCL microspheres decreases with a decreasing PCL concentration, increasing PVA concentration and increasing stirring rate used during microsphere preparation. The encapsulation efficiency of icariin in the microspheres was between 30 and $85 \%$. Very low drug loading and encapsulation efficiency was found for microspheres prepared with $10 \%$ (w/v) PCL solution. Possibly, stable microspheres were not formed at this low polymer concentration. In SEM images of these microspheres, string-like polymer precipitate was observed (data not shown). Furthermore, the drug loading and encapsulation efficiency decreased with an increase in the PVA concentration used during microsphere preparation. The PVA may have aided in solubilization of icariin in the aqueous phase, therefore reducing the drug particle loading into the microspheres.

Drug release from the microspheres into PBST was monitored over 7 days. The cumulative release profiles are shown in figure 3. 

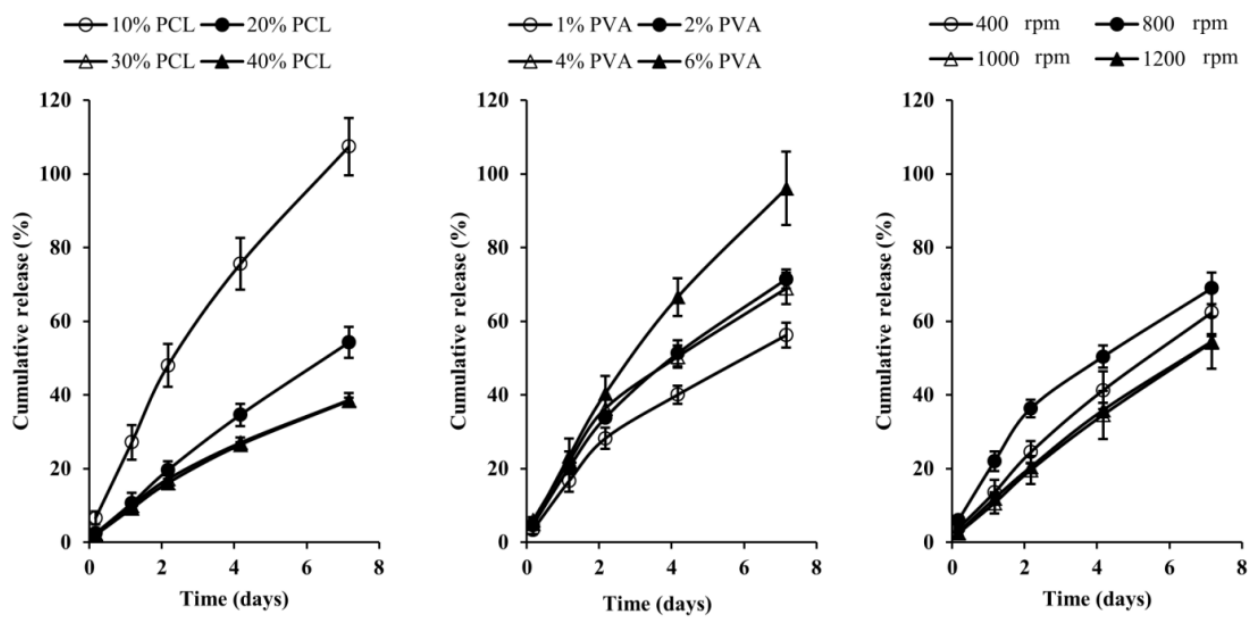

Figure 3. Cumulative icariin release from PCL microspheres. Relevant parameters adjusted during microsphere preparation are given in the legends above the graphs. Concentrations of PCL and PVA are given in $\% w / v$. For each variable parameter, the unchanged parameters were kept constant at 20\% (w/v) PCL concentration, $4 \%$ $(w / v)$ PVA concentration or an 800 rpm stirring rate (Table 1). Lines are depicted to guide the eye.

All microspheres released icariin in a controlled and sustained manner over 7 days. The release rate was mainly affected by the PCL and PVA concentrations used during microsphere preparation. With a decreasing PCL concentration and an increasing PVA concentration, the icariin release rate from the prepared microspheres was enhanced. The high release rate of icariin from the microspheres prepared with $10 \%(\mathrm{w} / \mathrm{v})$ PCL may be the result of their small size as well as the formation of the string-like precipitate observed in SEM. The increase of the release rate with increasing PVA concentration used during microsphere preparation may also be related to the decreasing size of the spheres. In addition, if the solubilization of icariin was indeed enhanced during microsphere preparation, it is likely that icariin was more concentrated in the outer part of the microspheres, and therefore was released more rapidly. The stirring rate used during microsphere preparation only slightly affected the release rate of icariin from the prepared microspheres.

Photo-crosslinked poly(trimethylene carbonate) and nHA composite structures with different nHA contents were loaded with icariin by a dip-coating method. The effect of the nHA content of the structures and the icariin concentration used during dip-coating on the loading of the composite structures was investigated. Figure 4 depicts the icariin loading of the structures. The nHA content in the composite structures enhances the icariin loading of the structures by dip-coating, ranging from $0.12 \mathrm{mg} / \mathrm{scaffold}$ for structures without nHA to $0.24 \mathrm{mg} / \mathrm{scaffold}$ for structures containing $47 \mathrm{wt} . \% \mathrm{nHA}$. Icariin loading could furthermore be controlled on structures with 47 wt. $\%$ nHA by the icariin concentration in the dipcoating solution. 

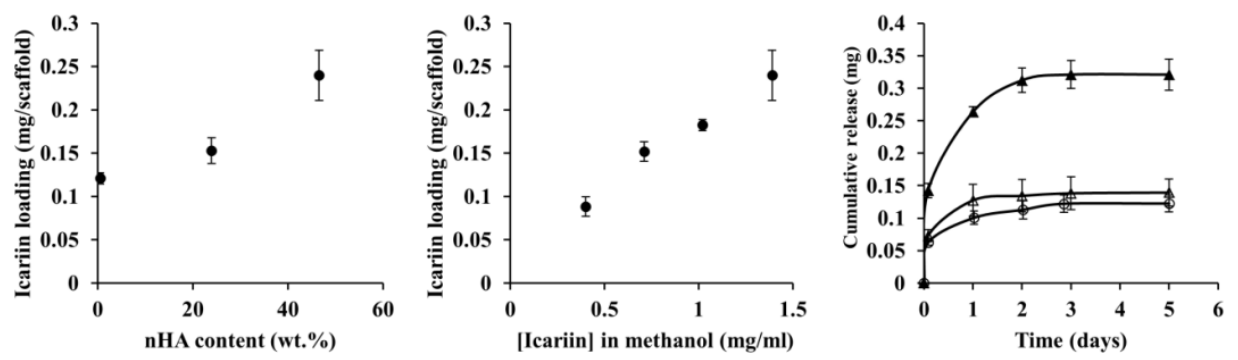

Figure 4. Icariin loading determined for photo-crosslinked poly(trimethylene carbonate) and nHA composite structures with different $n H A$ contents (left graph) and for structures loaded in icariin solutions of different concentrations (middle graph).Release of icariin from structures with 0 wt.\% nHA (open circles), 24 wt. $\%$ nHA (open triangles) and $47 \mathrm{wt} \%$ nHA (closed triangles) is depicted in the right graph. Lines are depicted to guide the eye.

The cumulative icariin release from photo-crosslinked poly(trimethylene carbonate) and nHA composite structures with different nHA content (loaded at $1.39 \mathrm{mg} / \mathrm{ml}$ icariin in methanol) was furthermore determined over 5 days and is also shown in figure 4 . With an increase of the nHA content in the structures, the icariin release rate was enhanced. This is likely due to the increased loading of icariin on the structures with increasing nHA content. A burst release of icariin was observed from all structures in the first 2 hours of release, after which a controlled release was observed up to 3 days. Thereafter, icariin was completely released from all structures.

\section{Conclusions}

Using solid-in-oil-in-water emulsions and dip-coating respectively, icariin loaded PCL microspheres and photo-crosslinked poly(trimethylene carbonate) and nHA composite structures were prepared. Parameters that influenced icariin loading and release were investigated and were used to control these characteristics. The icariin-loaded microspheres released icariin in a controlled and sustained manner over a prolonged period. These microspheres may find application by loading them into or onto implants for bone regeneration. Alternatively, dip-coated photo-crosslinked poly(trimethylene carbonate) and nHA composite structures may be used as well, as these showed a controlled release. The release was over a short period of time, however. 


\section{Appendix C - Composites based on photo-crosslinked poly(trimethylene carbonate) and microspheres}

\section{Introduction}

Microspheres have been successfully used for the protection and controlled delivery of medicine in vivo. Such microspheres may find application in restoration of bone defects when loaded with osteopromotive drug compounds. Direct application of microspheres in a bone defect is not practical however, as they do not possess structural integrity. A method to apply drug-loaded microspheres in a bone defect is by incorporating or attaching them to an implantable structure. Here we have prepared 3 types of microspheres, $\operatorname{poly}(\varepsilon-$ caprolactone) (PCL), gelatin and composite microspheres of gelatin and nanohydroxyapatite (nHA). These were encapsulated in a photo-crosslinked PTMC matrix and the mechanical properties of the resulting composites were investigated.

\section{Experimental}

Three-armed poly(trimethylene carbonate) with methacrylate end-group functionality (PTMC-MA) was synthesized as reported in Chapter 4 of this thesis. Using ${ }^{1} \mathrm{H}-\mathrm{NMR}$ (Bruker Ascend 400/Avance III $400 \mathrm{MHz}$ NMR spectrometer) the number average molar mass and degree of functionalization of PTMC-MA was determined to be $10.8 \mathrm{~kg} / \mathrm{mol}$ and $>99 \%$ respectively.

Microspheres (not containing icariin) were prepared from PCL as described in Appendix B. A PCL ( $\alpha-\omega$ hydroxyl group-terminated, $11 \mathrm{~kg} / \mathrm{mol})$ concentration of $20 \%(\mathrm{w} / \mathrm{v})$, a poly(vinyl alcohol) (PVA, 9-10 kg/mol, 80\% hydrolyzed) concentration of $4 \%(\mathrm{w} / \mathrm{v})$ and a stirring rate of $1200 \mathrm{rpm}$ were used during microsphere preparation.

Gelatin microspheres with and without nHA were prepared by a water-in-oil emulsion. Gelatin was dissolved in demineralized water at $15 \%(\mathrm{w} / \mathrm{v})$ and $80{ }^{\circ} \mathrm{C}$. For the preparation of gelatin microspheres containing nHA, $10 \% \mathrm{w} / \mathrm{v}$ of nHA was added to the solution. Thereafter, $5 \%(\mathrm{w} / \mathrm{v})$ of glucose was added and the resulting mixture was stirred for 5 minutes. It was thereafter added dropwise to soy bean oil stirred at $500 \mathrm{rpm}$ and $80{ }^{\circ} \mathrm{C}$. The resulting water-in-oil emulsion was stirred for 5 minutes to allow the glucose to further crosslink the gelatin. It was subsequently cooled to $5{ }^{\circ} \mathrm{C}$ and cold acetone was added to dehydrate the microspheres. The microspheres were recovered by filtration (Whatman, grade 597 filter paper) and were washed with acetone after which they were dried in vacuo at room temperature. Using TGA (Perkin-Elmer TGA7) it was determined that the composite gelatin microspheres contained $33 \mathrm{wt} . \%$ of nHA.

The microsphere diameter was determined from SEM images (JEOL JSM-IT100 SEM) using ImageJ analysis.

Stereolithography resins containing microspheres were prepared by addition of microspheres (10 wt.\% relative to PTMC-MA for PCL, 5 vol.\% relative to PTMC-MA for 
gelatin and its composite microspheres) to a PTMC-MA resin as reported in Chapter 6 of this thesis. Films were prepared by casting the resins on a glass plate at $500 \mu \mathrm{m}$ thickness and photo-crosslinking at $11 \mathrm{~mW} / \mathrm{dm}^{2}$ for 30 minutes under $\mathrm{N}_{2}$ atmosphere. Extraction and drying of the films was thereafter performed as described in Chapter 4. The resulting thickness of the films was between 550 and $580 \mu \mathrm{m}$ for films with PCL microspheres and between 350 and $450 \mu \mathrm{m}$ for films with gelatin and gelatin composite microspheres.

Mechanical properties of photo-crosslinked, microsphere-containing films were determine by tensile experiments using a Zwick Z020 tensile tester equipped with a 500-N load cell. The initial grip-to-grip separation was $50 \mathrm{~mm}$, and the crosshead speed was $50 \mathrm{~mm} / \mathrm{min}$. Sample dimensions were $100 \times 5 \mathrm{~mm}^{2}$.

\section{Results and discussion}

In figure 1 SEM images of the prepared microspheres are given. All of the microspheres were rounded in morphology. The PCL microspheres had a smooth surface morphology. On the surface of the gelatin microspheres, shallow pits were observed. The composite gelatin microspheres possessed a rough morphology. The average diameter of the PCL microspheres was $44 \mu \mathrm{m}$. The average diameter of the gelatin and the gelatin composite microspheres was 32 and $125 \mu \mathrm{m}$ respectively.
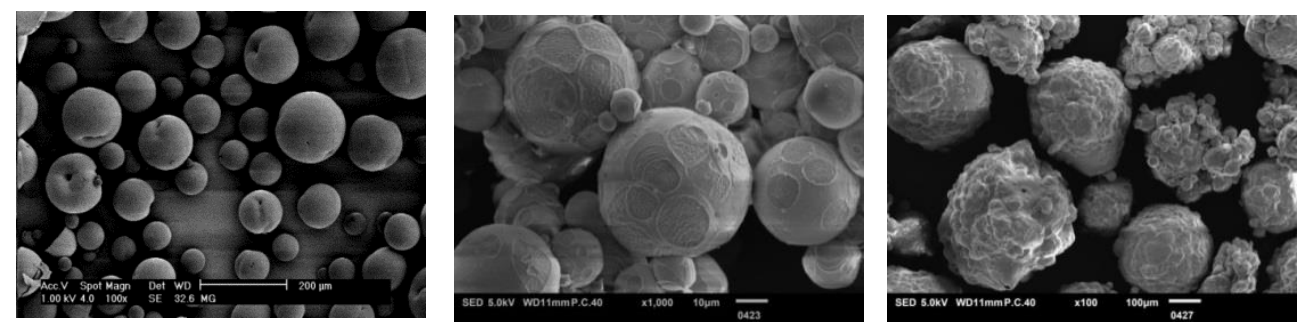

Figure 1. SEM images of the prepared PCL-(left image), gelatin- (middle image) and gelatin composite (right image) microspheres.

The mechanical properties of the photo-crosslinked films loaded with the different microspheres was determined by tensile experiments and are given in table 1. Tensile properties of films without microspheres are given for comparison and are taken from Chapter 4 of this thesis.

The tensile properties given in table 1 show that the composites of photo-crosslinked PTMC with microspheres have similar rigidities as the photo-crosslinked PTMC. The rigidity of the gelatin micro-spheres composite is a little higher. In all cases, the composite films are more brittle than the photo-crosslinked PTMC, having lower elongation at break and toughness. 
Table 1. Overview of mechanical properties determined for photo-crosslinked PTMC and photo-crosslinked PTMC with microspheres.

\begin{tabular}{lcccc}
\hline & \multicolumn{4}{c}{ Microspheres } \\
\cline { 2 - 5 } Property & None & PCL & Gelatin & Gelatin/nHA \\
\hline E modulus $(\mathrm{MPa})$ & $2.8 \pm 0.1$ & $2.7 \pm 0.1$ & $2.6 \pm 0.1$ & $3.3 \pm 0.1$ \\
Yield strength $\left(\mathrm{N} / \mathrm{mm}^{2}\right)$ & $0.6 \pm 0.03$ & $0.4 \pm 0.01$ & $0.5 \pm 0.02$ & $0.5 \pm 0.03$ \\
Elongation at yield $(\%)$ & $26 \pm 0.3$ & $20 \pm 1.0$ & $25 \pm 0.3$ & $20 \pm 0.3$ \\
Tensile strength & $2.4 \pm 0.3$ & $1.0 \pm 0.1$ & $1.2 \pm 0.1$ & $1.1 \pm 0.1$ \\
Elongation at break $(\%)$ & $239 \pm 33$ & $107 \pm 8.0$ & $154 \pm 19$ & $117 \pm 15$ \\
Toughness $\left(\mathrm{N} / \mathrm{mm}^{2}\right)$ & $330 \pm 71$ & $71 \pm 8.6$ & $127 \pm 22$ & $90 \pm 16$ \\
\hline
\end{tabular}

Although the brittleness of microsphere-containing composites was higher than that of photo-crosslinked PTMC, we have prepared three-dimensional composite structures with PCL microspheres as a proof of concept. These were fabricated by stereolithography, as was described in Chapter 6 of this thesis. In figure 2, SEM images of freeze-fractured cross-sections of photo-crosslinked PTMC and of its composite structure with PCL microspheres are depicted.
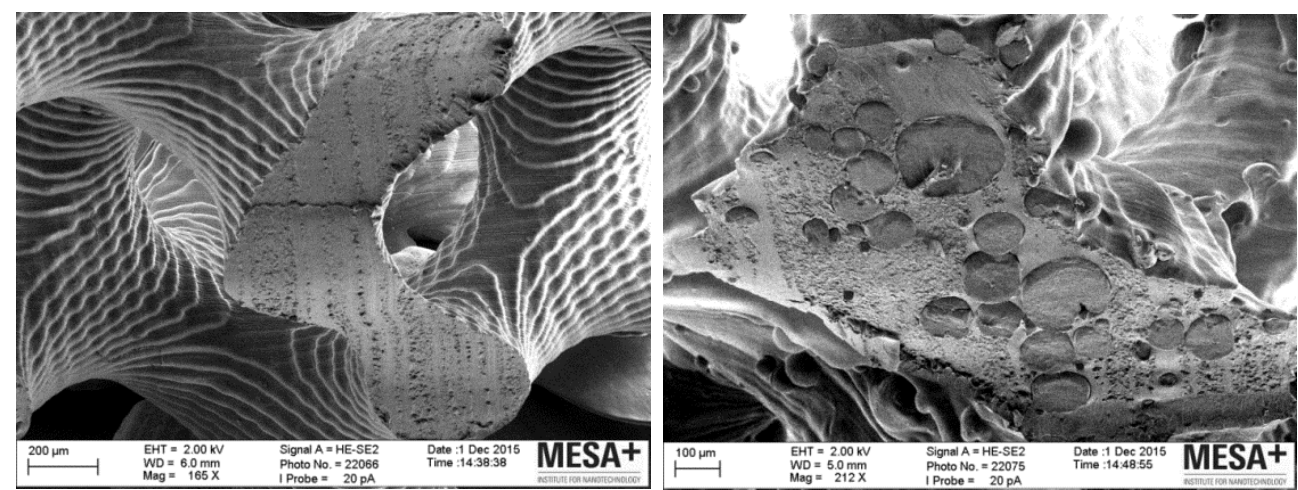

Figure 2. SEM images of the cross-section of structures prepared by stereolithography. The image on the left shows a photo-crosslinked PTMC structures, the image on the right shows a composite structure of photocrosslinked PTMC and PCL microspheres.

In figure 2 it can be observed that the photo-crosslinked PTMC structure is built up of sequentially polymerized layers. The cross-section of the structure was smooth. The surface of the photo-crosslinked PTMC structures containing PCL microspheres was rougher in morphology. Here too the sequentially photo-polymerized layers of the structure could be discerned. Microspheres attached to the surface of the structures were observed, although at some locations these were dislodged. In the cross-section, the microspheres were embedded within the photo-crosslinked PTMC matrix. 


\section{Conclusions}

Microspheres were prepared from PCL, gelatin and a gelatin composite with nHA. These microspheres were incorporated into photo-crosslinked PTMC to allow the preparation of a drug-releasing composite structure. The incorporation of the microspheres resulted in less tough materials. PCL microspheres were furthermore used to form three-dimensional composite structures with photo-crosslinked PTMC by stereolithography. This indicates that stereolithography may be applied to prepare composite structures loaded with drugcontaining particles as well. 


\section{Outlook}

In this work we describe the preparation and characterization of composites of photocrosslinked poly(trimethylene carbonate) (PTMC) and nano-hydroxyapatite (nHA), to meet the demand for novel implantable materials for bone tissue restoration.

These composites were prepared using conventional methods (solvent casting) and by additive manufacturing techniques (stereolithography and low temperature extrusion-based additive manufacturing). The nHA in the composites significantly improved their mechanical properties, hydrophilicity and degradability. Furthermore, it was shown that the surface of the composites became enriched with nHA by increasing the nHA content. This resulted in an enhanced surface roughness and subsequently, in an enhanced protein adsorption and calcium release. In vitro the composites were shown to be cytocompatible and allowed for a more effective differentiation of human bone marrow mesenchymal stem cells (hBMSCs) than photo-crosslinked PTMC without nHA. In vivo the composites effectively restored rabbit calvarial defects with good osseous integration when high nHA contents were used ( $\geq 40 \mathrm{wt} . \%$ ). Lastly, implantable structures with a multi-scale porosity were prepared. These may further enhance bone restoration in vivo.

Ideally, the composites reported here will find application in a clinical setting. A possible example is as patient-specific implants for craniomaxillofacial bone tissue restoration. Although the restoration of rabbit calvarial defects demonstrated the improvement of bone restoration efficacy by the inclusion of nHA in photo-crosslinked PTMC, it is not yet predictive for the performance in human patients. In fact, rabbits are known to be able to restore large bone defects autologously. Predicting the performance of the composites in a clinical setting for critical size bone defects will require more extensive in vivo experiments in larger animal models which mimic bone restoration in humans more closely. Currently, in vivo experiments in sheep are being evaluated. In these experiments the orbital floors of sheep were completely removed and replaced by 'sheep-specific', porous orbital floor implants. Furthermore, intramuscular implantations of porous composite structures were performed to determine whether the composites are osteoinductive. The evaluation of these experiments will yield valuable information to assess whether the composites are suitable for application in human patients.

In addition, it was shown in this thesis that surface enrichment of the composites by nHA may play an important role in the ability to restore bone defects. It is hypothesized that the enrichment is caused by the photo-crosslinking reaction used to prepare the composites and by the solvent extraction used to purify them. How this surface enrichment can be controlled is currently not clear, however. Comprehension of factors controlling this enrichment is necessary to ensure minimal batch-to-batch difference of the fabricated 
scaffolds. Also, it may offer further control and improvement over the bone restoring efficacy of the composite structures.

Lastly, in vivo experiments are required to evaluate the effect of a multi-scale porosity in the composites reported in this thesis. From this work it is known that enhancing porosity on the micron scale reduces the differentiation ability of hBMSCs into the osteogenic lineage. This is not indicative of the in vivo bone restoring capability of the composites with a multi-scale porosity, however. In literature it has been shown that a multi-scale porosity becomes more efficacious in restoring bone defects as the micro-porosity is enhanced. In vivo experiments of structures with a multi-scale porosity and varying porosity on the micron scale are required to assess the effect of the porosity in bone restoration.

Nonetheless, the photo-crosslinked PTMC and nHA composites introduced in this thesis have yielded promising results. The ability to process these composites into patient-specific structures with an interconnected and designed porosity and a multi-scale porosity makes them highly attractive for bone tissue restoration. Positive results in large animal studies open up possibilities for more extensive studies and ultimately, to human case studies.

The processing and application of photo-crosslinked PTMC and nHA composites is also not confined to the work reported in this thesis. Other formulations than resins for additive manufacturing may for example be attractive as well. Injectable, viscous dispersions of photo-crosslinkable PTMC and nHA or moldable pastes for direct application in defects are interesting options as well. These could be directly injected or compressed into defects and thereafter cured, offering patient specificity and direct integration into the surrounding tissue. Challenges lie in the non-toxic formulation of such materials and the ability to generate a porous structure after curing. 


\section{Summary}

Critical size bone defects need implantable structures to act as a temporary support and to induce de novo bone formation in the defect. Most synthetic implants are currently outperformed by natural bone grafts. The use of these grafts comes with several disadvantages, such as risk for disease transmittance, graft rejection and limited options for shaping. Thus, novel synthetic implants are required which do not possess the disadvantages of natural grafts and perform similarly well.

This thesis describes the preparation and characterization of a novel composite material of photo-crosslinked, methacrylate end-group functionalized poly(trimethylene carbonate) (PTMC-MA) and nano-hydroxyapatite (nHA). Specifically, this thesis describes the preparation of these composites by the use of additive manufacturing techniques and the characterization of the fabricated composite structures in terms of in vitro and in vivo performance as bone restoring implants.

In Chapter 1 a problem statement and the aim of this thesis are described alongside the outline of the work. Chapter 2 gives an overview of additive manufacturing techniques that can be used to prepare composites and the implications of processing composite materials for these techniques. A brief overview of methods for the design of porous implant structures is furthermore given. Finally, the progress that has been made in using additive manufacturing for the preparation of composite implants for bone tissue restoration is described by giving a literature overview.

In Chapter 3 composites were prepared by solvent casting films of PTMC-MA solutions with various nHA contents. After evaporation of the solvent the casted films were photocrosslinked and it was shown that the nHA was readily and homogeneously incorporated and that the photo-crosslinking was not significantly affected by the nHA content. The nHA did enhance the mechanical properties of the composites in terms of stiffness, tensile strength and toughness, which was also shown for composites prepared by stereolithography (Chapter 4 and Chapter 6).

It was furthermore shown in Chapter 4 that composites of photo-crosslinked PTMC-MA and nHA can be prepared in complex shapes by stereolithography. By using computed tomography data of actual patients with blow-out orbital floor fractures and a semiautomatic process for generation of fitting models of implants, it was possible to fabricate patient-specific orbital floor implant structures with various nHA contents. Apart from the mechanical properties of the composites it was also shown that their hydrophilicity is enhanced by increasing their nHA content.

This enhanced hydrophilicity partly led to an enhanced degradability with increasing nHA content, as was demonstrated in Chapter 5. Photo-crosslinked PTMC-MA was not significantly degraded at neutral- or acidic $\mathrm{pH}$ or by the action of cholesterol esterase. Only lipase had a slight effect. Composites containing nHA demonstrated a stronger degradation 
of the photo-crosslinked PTMC-MA matrix in enzyme solutions. It was hypothesized that by the introduction of nHA into the photo-crosslinked PTMC-MA matrix, degradation media could infiltrate the bulk of the composites. A high surface area of photo-crosslinked PTMC-MA matrix was therefore available for degradation by enzyme solutions. With increasing nHA content, the composites also became more susceptible to acidic degradation medium as nHA was dissolved. The results indicated that in an in vivo situation, the composites may be completely resorbable.

In Chapter 6 and Chapter 7, the biological performance of three-dimensional composite structures with an interconnected porosity was investigated. Using three different contents of nHA $(0,24$ and 47 wt.\%) it was shown that the surface of the composite structures was increasingly enriched with nHA as the nHA content was increased. Additionally, the composites structures became stiffer and more hydrophilic, were able to adsorb higher amounts of protein and released more calcium. Although this did not affect the attachment and metabolic activity of human bone marrow mesenchymal stem cells (hBMSCs) (Chapter 6) it clearly influenced their osteogenic differentiation as was shown by alkaline phosphatase (ALP) activity, ALP gene expression and by the calcium production of the cells (Chapter 7). Using the composite structures, bone growth in rabbit calvarial defects was significantly enhanced compared to structures of only photo-crosslinked PTMC. Although no significant differences were found between the amount of bone growing into the composites with 24 and 47 wt.\% nHA, there was a clear difference in the osseointegration of the structures. This demonstrated the benefit of including large amounts of nHA in the photo-crosslinked PTMC-MA composites.

The ability to prepare porous three-dimensional structures with a multi-scale porosity of photo-crosslinked PTMC-MA and nHA was demonstrated in Chapter 8. Using low temperature extrusion-based additive manufacturing and temperature induced phase separation; structures with a designed macro-porosity with pore sizes in the range of several hundred micrometers and a micro-porosity with pore sizes below ten micrometer were prepared. The resins used to prepare these structures show favorable properties for processing by extrusion-based techniques, such as shear thinning and a facile temperature induced phase separation. It was furthermore shown that specific drug molecules may be incorporated for a controlled drug release.

Temperature induce phase separation was also used in Chapter 9 to prepare photocrosslinked PTMC-MA composites with $40 \mathrm{wt} \%$ nHA and pores with sizes on the micron scale. The effect of varying the porosity on this scale on hBMSC attachment, proliferation and differentiation was investigated. Although enhancing the porosity enhanced surface roughness and protein adsorption on the composite surface, it significantly reduced its stiffness and reduced the ability of hBMSCs to spread into the morphology required for osteogenic differentiation. This ultimately resulted in a less pronounced osteogenic differentiation of the cells as was shown by their calcium production.

Overall, the work in this thesis shows that composites of photo-crosslinked PTMC-MA and nHA may indeed be useful in bone tissue engineering applications. Increasing nHA 
contents can be used to enhance the mechanical properties, hydrophilicity and the degradability of the composite. More importantly, it improves the bone regenerating capability. Lastly, the ability to prepare these composites by different additive manufacturing techniques is highly attractive as it allows for the fabrication of implantable structures with a designed external and internal architecture.

Additional work is needed to evaluate the composites in a more clinical setting and to evaluate the effect of a multi-scale porosity in the composite. Large animal studies in which the reconstruction of the orbital floor of sheep is investigated using 'sheep-specific', porous composite structures are currently evaluated. Additionally, intramuscular implantations of photo-crosslinked PTMC-MA and nHA composites are also being evaluated to assess the osteoinductivity of the composites. The in vivo implantation of composite structures with a multi-scale porosity should furthermore be performed to assess the effect of the porosity on bone regeneration. 


\section{Samenvatting}

Botdefecten van een kritieke afmeting benodigen implanteerbare structuren die als tijdelijke draagconstructie dienen en aanzetten tot de groei van nieuw bot. Synthetische implantaten worden dikwijls overtroffen door natuurlijk voorkomende botimplantaten. Deze natuurlijk voorkomende materialen bezitten meerdere nadelige eigenschappen, zoals een risico voor het overdragen van ziektes, afwijzing door het lichaam en een beperkte mogelijkheid tot het aanpassen van de vorm van het materiaal. Derhalve is er een behoefte naar nieuwe synthetische materialen waarbij de nadelige eigenschappen van natuurlijk voorkomende materialen afwezig zijn, doch de effectiviteit vergelijkbaar is.

Dit proefschrift omschrijft de bereiding en karakterisering van een nieuw composiet materiaal bestaande uit gefoto-crosslinkt poly(trimethyleencarbonaat) met methacrylaat eindgroepen (PTMC-MA) en nano-hydroxyapatiet (nHA). In het specifiek omschrijft dit proefschrift de bereiding van dergelijke composieten met behulp van additieve productietechnieken en de karakterisering van de bereide structuren doormiddel van in vitro en in vivo experimenten om zo de botherstellende eigenschappen te onderzoeken.

In Hoofdstuk 1 wordt de probleemstelling en het doel van dit proefschift geïntroduceerd, samen met een overzicht van het verrichte onderzoek. Hoofdstuk 2 geeft een overzicht van additieve productietechnieken welke bruikbaar zijn voor de bereiding van composieten en wat de gevolgen zijn van het verwerken van composieten voor deze technieken. Daarnaast wordt een kort overzicht gegeven van methoden om poreuze implantaten te ontwerpen. Tot slot wordt omschreven welke voortgang is geboekt in de literatuur omtrent de toepassing van additieve productietechnieken voor het bereiden van composiet implantaten voor botherstel.

Voor Hoofdstuk 3 zijn composietfilms bereid doormiddel van oplosmiddelgieten van PTMC-MA oplossingen met verscheidene nHA-gehaltes. Na verdamping van het oplosmiddel zijn de gegoten films gefoto-crosslinkt en is het aangetoond dat nHA moeiteloos en homogeen ingelijfd werd en dat het foto-crosslinken niet beduidend werd beïnvloed. Het nHA verbeterde de mechanische eigenschappen van het composiet in het kader van stijfheid, reksterkte en taaiheid van het materiaal. Hetgeen ook is aangetoond voor composieten bereid met behulp van stereolithografie (Hoofdstuk 4 en Hoofdstuk 6).

Daarnaast wordt in Hoofdstuk 4 gedemonstreerd dat composieten van gefoto-crosslinkt PTMC-MA en nHA in complexe vormen bereid kunnen worden doormiddel van stereolithografie. Daarbij is gebruik gemaakt van computertomografiegegevens van patienten met orbitavloerbreuken van een kritieke afmeting en een semi-geautomatiseerd procédé voor het genereren van passende orbitavloerimplantaten. Het was daarmee mogelijk om implantaten te fabriceren met verscheidene nHA-gehaltes, afgestemd op de betreffende patient. Naast verbeterde mechanische eigenschappen, leide een toenemend nHA-gehalte in deze composieten ook tot een verhoogde wateropname. 
Deze verhoogde wateropname was deels verantwoordelijk voor een toenemende afbreekbaarheid van de composieten met een toenemend nHA-gehalte, zoals gedemonstreerd is in Hoofdstuk 5. Gefoto-crosslinkt PTMC-MA brak niet significant af bij een neutrale of zure $\mathrm{pH}$ of door de werking van cholesterol esterase. Enkel lipase veroorzaakte een effect. Composieten met nHA vertoonden een sterkere afbraak van gefoto-crosslinkt PTMC-MA in enzymoplossingen. De hypothese was dat afbraakmedia in de bulk van de composieten zouden kunnen doordringen door de introductie van nHA in het gefoto-crosslinkte PTMC-MA. Een groot oppervlak van gefoto-crosslinkt PTMC-MA wordt daarbij blootgesteld aan enzymen in het afbraakmedium. De composieten werden daarnaast gevoeliger voor afbraak in medium met een zure $\mathrm{pH}$ met toenemend nHAgehalte, door de oplosbaarheid van nHA in zure media. Deze resultaten gaven aan dat de composieten compleet afbreekbaar kunnen zijn in een in vivo situatie.

In Hoofdstuk 6 en Hoofdstuk 7 werd de biologische effectiviteit van driedimensionale composiet structuren met een onderling verbonden porositeit onderzocht. Drie verscheidene nHA-gehaltes (0, 24 en 47 gew.\%) werden hierbij toegepast. Er werd aangetoond dat het oppervlak van de composieten in toenemende mate met nHA verrijkt werd naarmate het nHA-gehalte verhoogd werd. Bijkomend werden de composieten ook stijver en hydrofieler, lieten een grotere adsorptie van eiwitten toe en gaven meer calcium af. Alhoewel dit geen effect had op het hechten van menselijke mesenchymale stamcellen uit beenmerg (hBMSCs) (Hoofdstuk 6) aan de composieten, was er wel een duidelijk effect op de osteogene differentiatie van de cellen. Dit werd aangetoond doormiddel van kwantificering van de alkalische fosfatase (ALP) activiteit, ALP genexpressie en de calciumproductie van de cellen (Hoofdstuk 7). Door gebruik te maken van de composiet structuren was het verder mogelijk om botgroei in calvariale defecten in konijnen beduidend te bevorderen in vergelijking met structuren bestaande uitsluitend uit gefoto-crosslinkt PTMC-MA. Alhoewel er geen beduidend verschil was tussen de hoeveelheid botgroei in composieten met 24 en 47 gew.\% nHA, was er een duidelijk verschil in de osseointegratie van de structuren. Dit demonstreerde de voordelen van het inlijven van grotere hoeveelheden nHA in de gefoto-crosslinkte PTMC-MA composieten.

De mogelijkheid tot het vormen van driedimensionale structuren van gefoto-crosslinkt PTMC-MA en nHA met een porositeit op meerdere schalen van grootte is gedemonstreerd in Hoofstuk 8. Gebruik makend van een op extrusie gebaseerde additieve productietechniek bij lage temperatuur en fasescheiding doormiddel van temperatuursverandering, zijn structuren bereid met een ontworpen macro-porositeit met poriegroottes op een schaal van meerdere honderd micrometer en een micro-porositeit met poriegroottes beneden tien micrometer. De benutte harsen voor de bereiding van deze structuren vertonen gunstige eigenschappen voor verwerking met behulp van op extrusie gebaseerde technieken, zoals afschuifverdunning en een moeiteloze bewerkstelliging van fasescheiding doormiddel van temperatuursverandering. Daarnaast is gedemonstreerd dat een gecontroleerde medicijnafgifte uit het bereide composiet mogelijk is. 
Fasescheiding doormiddel van temperatuursverandering is ook benut in Hoofstuk 9 om gefoto-crosslinkte PTMC-MA composieten te bereiden met 40 gew.\% nHA en poriën met een afmeting op de micrometer schaal. Het effect van porositeitsaanpassingen op deze schaalgrootte op de aanhechting, vermenigvuldiging en differentiatie van hBMSCs is onderzocht met deze composieten. Verhoging van de porositeit leidde tot een verhoogde oppervlakteruwheid en adsorptie van eiwitten, doch verlaagde de stijfheid van het composiet en beperkte de mogelijkheid voor hBMSCs om een morfologie aan te nemen welke benodigd is voor osteogene differentiatie. Dit resulteerde in een verminderd uitgesproken differentiatie van de cellen, zoals aangetoond door het kwantificeren van calciumproductie.

In grote lijnen toont dit proefschrift aan dat composieten van gefoto-crosslinkt PTMC-MA en nHA wezenlijk bruikbaar kunnen zijn voor toepassingen in botherstel. Het verhogen van de nHA-inhoud in de composieten kan daarbij benut worden om de mechanische eigenschappen, hydrofiliciteit en afbreekbaarheid te verhogen. Van groter belang is dat het zorgt voor een verbeterd botherstel. De mogelijkheid om de composieten met behulp van additieve productietechnieken te bereiden is daarnaast uiterst aantrekkelijk, gezien het gelegenheid geeft tot het fabriceren van implantaten met een ontworpen externe en interne structuur.

Aanvullend onderzoek is benodigd om de composieten in een klinische omgeving te toetsen en om het effect van porositeit op meerdere schalen van grootte te beoordelen. Momenteel zijn experimenten voor het herstel van orbitavloeren in schapen reeds uitgevoerd met 'schaap-gepersonaliseerde' orbitavloer implantaten en worden de uitkomsten van deze experimenten verwerkt. Bovendien zijn intramusculaire implantaties van gefoto-crosslinkte PTMC-MA en nHA composieten uitgevoerd om de osteoinductiviteit te beoordelen. Voorts is het nodig om in vivo implantaties uit te voeren van structuren met porositeit op meerdere schalen van grootte om zo het effect van de porositeit op botherstel te beoordelen. 


\section{Acknowledgements}

During my time as a $\mathrm{PhD}$ student, I was lucky to meet many helpful and sociable people that made this period even more enjoyable than it potentially was already. Hereby I would like to express my gratitude to all those people.

First, I cannot go without thanking Prof. Dirk Grijpma. The day that you visited the chemistry lab of Developmental BioEngineering to tell me that you are looking for a $\mathrm{PhD}$ student, was a surprising one for me. Just two or three days before I actually decided to look for a $\mathrm{PhD}$ position. After giving it some thought and discussing the project in more detail, I decided to pursue the position. Looking back, I think I couldn't have made a better choice. The talks we had in a professional setting were always fruitful and I sincerely enjoyed our talks outside of this setting as well, on many of the trips we made for the project. Throughout my $\mathrm{PhD}$ project, you showed me that I could often be more confident in my abilities. Hereby I want to thank you for offering me the project and for all your guidance over the past years.

Next, I want to thank Prof. David Eglin and Dr. Olivier Guillaume with whom I have had the pleasure of working with. In part for the great contribution you have made to this thesis, but also for the excellent time I had in your labs at the AO Research Institute. During my time in Davos I always felt very welcome and I learned a lot (although, Olivier, I have to admit that I do not remember the dilution of stock components required for full osteogenic medium from the top of my head anymore). Thank you both very much for all the arrangements you made for me, your hospitality and all the good times we had inside and outside the AO. Additionally, I thank you for taking place in my graduation committee.

My thanks also go out to Prof. Geoff Richards and Prof. Mauro Alini for receiving me in the AO Research Institute. Both of you made me feel even more welcome in Davos and made our project meetings additionally enjoyable.

Prof. Joost de Bruijn, Dr. Huipin Yuan and Dr. Davide Barbieri, thank you very much for your contribution to this work as well as our conversations which helped me understand important aspects of working with calcium phosphates in general and nano-hydroxyapatite specifically. Prof. Joost de Bruijn, additional thanks go out to you for taking place in my graduation committee.

Many thanks also go to Prof. Ton Peijs, Dr. Julien Gautrot and Dr. Xi Zhang. It was a pleasure to collaborate with you on the great work you performed with photo-crosslinked composites of poly(trimethylene carbonate) and electrospun fibers.

Prof. Ling Qin, Dr. Yuxiao Lai, Prof. Ting-Ting Tang, Dr. Xinjiang Wang and Prof. Jiang Peng, thank you very much for our collaboration. Additional thanks to Prof. Ling Qin and Prof. Ting-Ting Tang for hosting some very nice meetings in China and for the great tours 
and dinners in and around Hong Kong, Shanghai and Xi'an. Special thanks also go to Ye Li for showing me around in Shenzhen, Hong Kong and Shanghai together with your friends from the Shenzhen Institutes of Advanced Technology and the Chinese University of Hong Kong.

Thank you Prof. Ruud Bos for the good company in Davos, for sharing with me your knowledge on orbital floor reconstruction and for a great day of skiing.

My gratitude also goes out to my other committee members not yet mentioned. Thank you, Prof. Giovanni Vozzi, Prof. Theo Smit, Prof. Pascal Jonkheijm and of course Prof. Hans Hilgenkamp as chair of my committee. Your time and effort spent on evaluating my work is greatly appreciated.

Of course I also want to thank the many members of the Biomaterials Science \& Technology group. To start off, I want to extend many thanks to Aysun. With you there was never a dull moment. Whether it was lunch, coffee or (passive) smoking time, I always enjoyed it. This was surely due to your joyful, exploring and kind nature. Our extensive exploration of Utrecht by bicycle (I still feel a bit sorry for letting you take your bicycle in the train there), our 'soccer matches' in the Zuidhorst hallways (which officially never happened) and me saving a drone from a pine tree are just a few examples of our memorable activities together. Unluckily, your Turkish lessons were a bit wasted on me. Still, I hope to be able to pronounce 'biberli tavuk salam' with the correct intonation soon. In some time you will also finalize your time as a $\mathrm{PhD}$ student and I sincerely hope that you will find your new dream position. Hopefully the Dutch weather will also be more kind to you around that time. Otherwise you'll have to come to Genova; hope to welcome you there soon!

Many thanks also go to my office mates with whom I have had the pleasure to reside in the 'boss office'. Bas, Erwin and Frits, you welcomed me into the office in the very beginning of my PhD. Surely, I was lucky to be in the office with you, where good music (even the occasional 'harde jongens uit de buurt'), good coffee (maybe the tea was good as well) and excellent calendars were well-represented. Also I want to thank you for making me 'grow up' and show some interest in whisky. Even though I'm still not very knowledgeable about it, I greatly enjoyed the whisky evenings. With a bit of luck we can get together again in the future. Bas, thank you additionally for our discussions which helped me plan some nice experiments and also for bringing some distractions during work hours in the form of trivia or just plain fun. Also thank you for planning some of the cycling trips we made. Hopefully we will meet soon in Finland and/or Italy.

Nick, thank you as well for sharing the office with us and spending some good times together. It was a shame that you left to Maastricht, although luckily we met several times after. Almost as important: thank you for giving new life to our office plant.

Ruchi, your presence in the office was much valued as well. We always had some nice chats and you were luckily always appreciative of a bit of background music. 
As my office mates I also want to thank Arnemieke and Honglin for the time we spent in the final months of my $\mathrm{PhD}$.

Many thanks I want to extend for nice conversations and some good times spent together to Natalia, Denys, Rachel, Tony, Seb, Aga, Kasia, André, Dimitrios, Jos, Bade, Iris, Acarilia, Pia, Ilaria, Thijs, Lydia, Rinske, Magda, Jai, Jonas, Praneeth, Jonathan, Odyl, Doo-Li , Jia, Piet, Duco, Marcel and Dwi.

Pia, thank you additionally for being my 'skiing instructor' the first time I went on Jakobshorn in Davos. Although I didn't do that well on the piste, I really appreciated your help.

Iris, I want to extend also some additional thanks to you. Even though you may have sometimes felt like you were 'bothering' me with your synthesis questions, I often enjoyed our conversations. Most of the times I learned something myself while we were discussing. Surely, you will keep on making some nice and novel polymer networks that I can read about soon.

Special thanks go to Zhengchao, Karin, Marc, Hetty, Anita and Zlata. Zhengchao, you helped a lot in finalizing the degradation work in chapter 5. Thank you very much for that. Karin, thank you for all your help in organizational matters during my $\mathrm{PhD}$ and even after my contract finished. You were a great help. Apart from the fact that you helped me, it was always nice to have some leisurely talks whenever I stepped into your office with one of my 'problems'. Marc, it is only 'fair enough' to thank you for your expertise on the lab and moreover for the many conversations on every-day topics, which made working on the labs even more enjoyable. Hetty, thank you for your help on several experiments which I couldn't have done without you. Anita, also you helped me a lot with some experimental work on the lab, thank you for that. Zlata, thank you for your help whenever I had to acquire some exotic chemical or equipment. Anything I could come up with, was apparently never exotic enough (you were always able to have it in the Zuidhorst within 2 weeks or less).

During the time I spent at the AO Research Institute, I met many (or actually, only) nice people to which I want to extend my thanks as well.

Gert-Jan, thank you very much for the great times we spent together in Davos and for introducing me into the Lunch Group and the exciting Davos nightlife. It was always good to have you around for a chat about anything and I greatly enjoyed the nice trips you organized to Rinerhorn and Schatzalp. Also, I want to thank you for your patience during my mediocre first descent from Jakobshorn. Luckily I redeemed myself a bit the year after, when we went skiing with Prof. Ruud Bos. Hopefully we can meet again soon, if not in The Netherlands, maybe on a trip through the sunny Puglia.

Dalila, I want to thank you as well for the good times spent together in Davos and most recently in B'taunt (please imagine the correct Bitonto accent while reading it). We were always able to joke around a bit in the lab, which made some of the longer and more difficult days easier. By now you will have acquired the title of Dr. and are ready to start 
enjoying the remarkable combination of Italian and Swiss lifestyle in Lugano. In any case, I wish you all the best and I'm sure we will meet soon in Genova or another nice city/town in Italy.

Also I want to thank Dr. Christoph Sprecher without whom I wouldn't have been able to perform many of the experiments I did in Davos. Your joyful nature and willingness to help whenever possible made it a pleasure to work with you.

Thanks also to Reihane, Stijn, Marina, Anamaria, Yanek, Alejandro, Marc Daniel, Rose, Simona, Federico and Dominic for all the awesome lunches we shared. It made me happy to enjoy your culinary treats as well as drinks together in Chämi or La Onda.

During the past four years I was lucky enough to have supervised two student projects which contributed to this thesis. Järvi, you did a lot of work in just three months on preparing icariin-loaded microspheres. Your explorative work showed me the opportunities and limits this drug delivery system had to offer. Thank you for that.

Anna, when you came to Enschede from Pisa for your master thesis work, I think we both had slightly different expectations. You being a Biomedical Engineering student, I expected a perfect match for the project I had thought of. It would involve some polymer synthesis, drug loading and release, additive manufacturing and in vitro characterization. You, however, were quite surprised to start the project with synthesis as you didn't have practical experience in this field. Nonetheless, it was not difficult for you to adapt. While working in the lab you were even able to endure all the 'why are you doing it in this way?' and always had the one perfect answer for it. Your contribution to chapter 9 of this thesis was great and will hopefully lead to a publication soon. Thank you.

In these acknowledgements my fellow 'bedrijfshulpverleners' (or more fancily: Emergency Response Officers) also deserve a place. It was great to have been on the team and I really enjoyed the trainings and outings we had together. Much less I enjoyed the occasional piece of bread that was turned into black smoke in the Meander microwaves, setting off the fire alarm. For the Meander population it must have been equally enjoyable to be evacuated afterwards though. In any case, it was part of the job and I learned a lot while doing it. Tyrone, Bert, Henk, Carla, Ivo, Geert, Walter, Cock, Dries, Wouter, Thomas, Fjodor, Nicolai, Leo, Marcel, Robert Me, Robert Mo, Timon, Steven, Patrick L, Patrick W, Katja, Dennis and Ronald, thank you for the excellent time I had on the team.

My housemates, Jefry, Bouke, Marnix, Mees and Gijs I want to thank for all the random relaxing evenings we spent in the living room and in the Vestingbar. The last of which could not always be defined as relaxing, but were just great fun. It's a big loss for me not being able to watch all the 'ouwelulle programma's' such as 2 Voor 12, Kassa, Per Seconde Wijzer, Keuringsdienst van Waarde or occasional LuckyTVs (wooooow, overal....dieren!!!!) with you guys and a big mug of coffee. All the best to you and hope to see you again (for another Vestingbar party maybe?). 
Coming close to the end of these acknowledgements, I want to thank my parents. Mam, pap, jullie hebben me altijd ondersteund. Toen dat heel erg nodig was voordat ik ging studeren, tot nu. Door jullie steun heb ik het gevoel me zo goed mogelijk te hebben ontwikkeld (niet alleen op professioneel vlak natuurlijk) en ik weet dat ik altijd op jullie kan rekenen. Heel erg bedankt!

Naast mijn ouders wil ik ook de rest van mijn familie bedanken. Jullie waren altijd erg geïnteresseerd in mijn promotieonderzoek en ik heb het altijd mooi gevonden jullie hierover iets te vertellen.

Last and most definitely not least, I want to thank Anna for a second time in these acknowledgements. In this case not for your accomplishments as a student, but for more personal reasons. The circumstances under which we met may not have been ideal, but looking back I have no regrets whatsoever. It makes me really happy to call you my friend+++++++++++++++++++++++++++++++++++++++++; more commonly known as 'girlfriend'. Although we haven't been together for a long time now, I want to thank you for the great time we had, are having and will surely have together. Love you a lot!

Mike 

Critical size bone defects are challenging to repair and current methods such as bone graft transplantation are characterized by drawbacks like disease transmittance, graft rejection and limited shaping options. Synthetic composite implants may provide an alternative for restoring bone defects without the drawbacks associated to bone grafts.

In this thesis, a novel composite of photocrosslinked poly(trimethylene carbonate) and nano-hydroxyapatite is investigated for its bone restoring capabilities. It is shown that the incorporation of nano-hydroxyapatite in the composites can be used to modulate the mechanical properties, hydrophilicity and degradability. Using different additive manufacturing techniques, designed and patient-specific composite implants can be prepared. These implant materials may be applied in vivo to enhance restoration of bone defects.

ISBN: 978-90-365-4543-3 